Revue belge de géographie

\title{
Progrès de la connaissance du Congo, du Rwanda et du Burundi de 1993 à 2008
}

\section{Henri Nicolaï}

\section{(2) OpenEdition}

1 Journals

\section{Édition électronique}

URL : https://journals.openedition.org/belgeo/7306

DOI : $10.4000 /$ belgeo.7306

ISSN : 2294-9135

Éditeur :

National Committee of Geography of Belgium, Société Royale Belge de Géographie

\section{Édition imprimée}

Date de publication : 31 décembre 2009

Pagination : 247-404

ISSN : 1377-2368

\section{Référence électronique}

Henri Nicolaï, « Progrès de la connaissance du Congo, du Rwanda et du Burundi de 1993 à 2008 »,

Belgeo [En ligne], 3-4 | 2009, mis en ligne le 12 mars 2013, consulté le 21 septembre 2021. URL : http:// journals.openedition.org/belgeo/7306 ; DOI : https://doi.org/10.4000/belgeo.7306

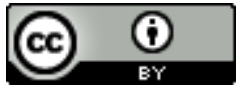

Belgeo est mis à disposition selon les termes de la licence Creative Commons Attribution 4.0 International. 


\title{
Progrès de la connaissance du Congo, du Rwanda et du Burundi de 1993 à 2008
}

\author{
Henri Nicolaï \\ Université Libre de Bruxelles
}

\begin{abstract}
RÉSUMÉ
Cette chronique, la vingt et unième d'une série qui a commencé avec l'année 1949, couvre la période 1993-2008 et a pour objectif de faire le point sur les progrès réalisés sur la connaissance du Congo (République démocratique du Congo), du Rwanda et du Burundi, dans le domaine de la géographie mais aussi dans les domaines des sciences naturelles et des sciences humaines qui peuvent fournir des données utiles ou indispensables aux géographes. Chaque référence bibliographique, livre ou article, est accompagnée d'un bref commentaire qui en retient les éléments principaux et surtout les faits ou les idées qui intéressent particulièrement les géographes. L'article comporte onze chapitres dont les plus importants concernent le milieu naturel, la géographie de la santé, la démographie, I'histoire (y compris l'histoire récente), la vie sociale et économique des campagnes traditionnelles et modernes, le secteur informel, les aspects de la vie urbaine. Les événements dramatiques qui se sont produits dans ces territoires africains au cours des quinze dernières années ont rendu la recherche sur le terrain particulièrement difficile tant pour les chercheurs nationaux que pour les chercheurs étrangers, ce qui se traduit notamment par une part de plus en plus importante des recherches menées en milieu urbain.
\end{abstract}

MOTS-CLÉS: Congo, Zaïre, Rwanda, Burundi, bibliographie commentée

\begin{abstract}
This paper, the $21^{\text {st }}$ issue of an edition of books and paper reviews on the knowledge of three countries of former Belgian Africa (DR Congo, Rwanda, and Burundi), covers the period 1993-2008. A short text for each reference points out the facts or ideas that are useful for geographers. The paper is composed of eleven sections. The most important are coping with the natural environment, health geography, population geography, history (including recent events), social and economic life in traditional and modern rural areas, informal economy, and urban geography. During the last sixteen years, conditions for field researches have become particularly difficult because of the warfare in the eastern part of the region and because of the economic collapse.
\end{abstract}

KEY WORDS: Congo, Zaire, Rwanda, Burundi, commented bibliography 


\section{INTRODUCTION}

es derniers Progrès de la connaissan- ce géographique du Congo, du Rwanda et du Burundi s'arrêtent à l'année 1992 (Nicolaï H., 1993). Nous avons compilé, dans un volume publié en 1996 (Nicolaï H., Gourou P., Mashini Dhi Mbita Mulenghe), les références concernant le Congo contenues dans les vingt articles publiés depuis l'année 1949. Depuis lors, si nous avons continué à nous occuper des rubriques Congo/Zaïre, Burundi et Rwanda dans la Bibliographie Géographique Internationale, pour des matières relevant explicitement de la géographie, nous n'avons plus fait le point général des connaissances. II nous a semblé utile de le faire maintenant.

Nous rappelons au lecteur que notre démarche n'est pas d'établir un dépouillement exhaustif mais de présenter l'état d'avancement de la recherche géographique dans ces pays en informant en même temps les géographes des progrès réalisés dans des domaines qui peuvent leur fournir des données utiles ou indispensables à leurs propres travaux et à leurs réflexions. Des domaines entiers n'ont donc pas été explorés comme celui de la linguistique où la recherche est importante mais dont nous ne retiendrons éventuellement que ce qui est susceptible d'éclairer des questions historiques ou d'identité ethnique.

Le lecteur aura donc toujours à l'esprit que notre bibliographie est lacunaire, qu'elle ne comporte que les ouvrages dont nous avons eu connaissance et que, parmi ceux-ci, nous avons procédé à un choix qui n'est pas nécessairement fondé sur la qualité intrinsèque de l'ouvrage ou de l'article mais sur l'intérêt qu'il peut présenter pour un géographe.

Certaines rubriques paraîtront plus fournies que dans les chroniques précédentes, d'autres au contraire beaucoup plus pauvres. C'est que les quinze der- nières années n'ont guère été propices aux études de terrain étant donné les événements tragiques qui se sont produits depuis le début des années 1990. Ceuxci ont gêné ou empêché la poursuite de recherches ailleurs que dans les villes tant pour les chercheurs autochtones que pour les chercheurs étrangers. Un ouvrage consacré à la méthodologie des recherches qui ont été menées au Congo depuis 1990 (c'est-à-dire depuis le début de la «transition politique»), l'explique aussi par le fait que la population urbaine est devenue de plus en plus importante et que les bailleurs de fonds ont privilégié l'étude des problèmes aigus que connaissent les grandes villes, accentuant ainsi la perception négative du milieu rural en matière de développement (Trefon Th. et Petit P., 2006, pp. 19-31).

D'autre part, au sein du monde congolais de la recherche et au sein de l'Université en particulier, les conditions matérielles ont été moins favorables encore que pendant les périodes précédentes. Le nombre des jeunes géographes ne s'est pas réduit mais les moyens dont ils ont pu disposer ont été loin de croître. La vie des universités est difficile. Le cursus académique de l'étudiant est un parcours semé d'embûches et très long. De 1990 à 2001, il y a eu, à l'Université de Kinshasa, cina années «blanches», comme on les appelle là-bas, c'est-à-dire des années qui n'ont pas pu être clôturées par un examen, donc qui ont été perdues pour l'étudiant. II y a eu aussi des années «plastiques» c'est-à-dire s'étendant sur 15 mois. Par conséquent, un étudiant qui a commencé ses études à l'âge de 20 ans et qui n'a redoublé aucune année ni changé de programme d'études est devenu licencié à 31 ans, alors que le cursus ne compte normalement que cinq années d'études (Ngub'usim Mpeg Nka R., 2002). 
En ce qui concerne les géographes africains en particulier, pas plus aujourd'hui qu'il y a quinze ans, ils ne disposent d'instruments suffisants pour faire connaître leurs travaux. L'absence de revues propres ayant une diffusion suffisante ou internationale et surtout régulière, y est pour beaucoup. Certains supports ont même disparu comme le Bulletin du CERUKI, dans le Kivu, qui avait été particulièrement actif dans les années 80. Ou bien ce sympathique Bulletin géographique de Kinshasa-Géokin mis en oeuvre par quelques Congolais basés en Belgique. Les publications facultaires éprouvent les plus grandes difficultés à subsister. Pour la diffusion des informations, le recours à l'Internet est encore balbutiant.

Les articles publiés dans des revues européennes ou américaines sont dus le plus souvent à des chercheurs qui ont fréquenté des universités occidentales pour obtenir un certificat, une maîtrise ou un doctorat et y séjournent parfois encore. Ils ne donnent donc qu'une idée très partielle de ce qui se fait dans l'université africaine même et des débats qui l'animent. Le nombre de mémoires de licence présentés chaque année est considérable. Si nous prenons un thème géographique précis, comme la menace et les conséquences de l'érosion dans les différents quartiers de Kinshasa, nous trouvons dans la bibliographie d'un livre récent sur les problèmes d'environnement (Lelo Nzuzi, 2008) au moins douze mémoires depuis la fin des années 1990 et davantage encore pour les problèmes liés à l'abandon des déchets solides sur la voie publique et dans les ravines. Mais aucun n'a été publié. Par contre, dans le domaine des sciences historiques, politiques ou sociales au sens large, de nombreux travaux ont été accueillis par des maisons d'édition françaises (L'Harmattan et Karthala) qui offrent de larges possibilités aux auteurs africains et qui publient aussi très souvent les actes de colloques organisés généralement en Europe ou en Afrique auxquels ceux-ci ont pu participer.

L'arrêt de la coopération belge, du moins sous la forme qui était la sienne avant 1990, a été un autre facteur négatif. II y a aujourd'hui un désintérêt apparent pour les recherches menées par les Belges en Afrique centrale. II s'exprime parfois de façon curieuse. C'est par exemple le faible pourcentage des recherches et des publications consacrées à l'Afrique centrale dans les rapports annuels du Département de Géologie et de Minéralogie du Musée royal de l'Afrique Centrale qui pourtant, par définition ou par tradition, devrait s'en occuper prioritairement. Dans le rapport des années 1999-2000, sur les trente-deux publications du département, une seule se réfère spécifiquement, dans son titre, à l'Afrique centrale. Sur les 31 notes indiquées comme en cours d'édition, 7 seulement, soit moins d'un quart, et 14 sur les 86 communications présentées à des colloques. Des trois notes originales figurant dans le même rapport, une seule concerne cette partie de l'Afrique (elle discute les caractéristiques morphologiques d'un poisson téléostéen du Jurassique moyen), une autre est marginale (Tanzanie occidentale), la troisième se rapporte au Ghana. Cela donne l'impression que les chercheurs ont dû se rabattre sur d'autres territoires. Le cas du fossé du lac Tanganyika est abordé mais à titre de comparaison avec celui du lac Baïkal; l'étude commune des deux fossés faisant l'objet d'un projet international où semblet-il, jusqu'à présent, le fossé asiatique a été privilégié.

Les projets des chercheurs belges se sont diversifiés géographiquement et couvrent aussi d'autres pays d'Afrique, d'Amérique latine et d'Asie. Par conséquent, le nombre des projets sur l'Afrique centrale a diminué d'un point de vue relatif mais aussi en valeur absolue. Ainsi la revue GEO-ECO-TROP (Revue de Géographie, d'Ecologie et de Géologie tropicales), au cours de ces dernières années, a publié autant sinon davantage d'articles sur l'Afrique du Nord que sur l'Afrique tropicale.

Nous n'avons pas cherché à combler les lacunes de nos bibliographies précédentes. Aucune référence ne sera donc antérieure à 1993 sauf dans le cas où une publication était nécessaire pour comprendre les publications qui l'ont suivie. 
On remarquera sans doute qu'un nombre non négligeable de publications peuvent apparaître comme des doublons, surtout pour celles qui traitent directement ou indirectement de faits politiques. C'est que les auteurs ont été sollicités à publier des articles sur les mêmes thèmes dans des revues différentes. Ils les ont publiés aussi dans d'autres versions linguistiques. La rapidité des évolutions les a contraints souvent à compléter ou à réviser des articles antérieurs.

Une autre remarque générale: nous n'avons pu passer sous silence les événements politiques et tout particulièrement les événements sanglants du Rwanda, du Burundi et du Kivu. Leurs conséquences ont été énormes pour la géographie humaine de ces pays. Le génocide rwandais a ébranlé la conscience du Monde. Des scientifiques mais aussi des journalistes, des écrivains, des hommes politiques, des moralistes, des cinéastes ont décrit et cherché à expliquer la tragédie. Ce sont des reportages, des témoignages (des victimes comme des assassins), des récits documentaires, souvent de violents réquisitoires qui ciblent des hommes, des groupes, des pays. Les cinéastes ont puisé des thèmes dans les guerres civiles africaines et même dans celui des épidémies virales. Ces thèmes ont succédé ainsi aux anciens mythes véhiculés par le cinéma occidental sur l'Afrique tropicale et qui mettaient surtout en scène des personnages européens: Tarzan, les aventuriers chercheurs d'or ou d'ivoire, les chasseurs capturant les grands animaux sauvages (ou dans leurs derniers avatars, les scientifiques étudiant ces animaux, vivant parmi eux et victimes parfois des braconniers). De ce foisonnement, nous essaierons de retenir surtout les publications qui analysent soit certains facteurs explicatifs de type géographique soit les conséquences des événements pour la démographie, la géographie humaine et économique ou l'environnement.

\section{GÉNÉRALITÉS}

\section{LE DÉBAT SUR LA RECHERCHE AFRICA- NISTE EN BELGIQUE}

La pertinence de recherches africanistes en Belgique, surtout dans le domaine des sciences humaines, nourrit depuis longtemps des débats. Certains les justifient par le sentiment d'un devoir à remplir, celui de conserver et de diffuser un patrimoine scientifique constitué pendant toute la période coloniale. Cette question doit évidemment tenir compte des sentiments et même des revendications légitimes de la partie congolaise. Elle a été rendue plus complexe encore par les tensions qui se sont manifestées dans les relations entre les Etats. Un très gros volume des Cahiers Africains lui a été consacré au moment où ces tensions atteignaient un de leurs points culminants (de Villers G., 1994). On y trouvera notamment des réflexions sur les ressources scientifiques, culturelles et humaines de l'africanisme en Belgique dans les communications de J.-L. Vellut et de Ndaywel è Nziem. G. de Villers a décrit les péripéties qui ont conduit à la création de l'Institut Africain, qui s'est inscrit un moment dans les préoccupations de certains hommes politiques s'occupant de la coopération au développement (de Villers G., 1995). Cet Institut est devenu finalement une section du Musée Royal de l'Afrique Centrale sous la dénomination «Histoire du temps présent». C'est cette section qui poursuit la publication de la très riche série des Cahiers africains, édités en collaboration avec L'Harmattan. Signalons, par les milieux universitaires d'Anvers, la publication d'un Annuaire de la Région des Grands Lacs. La revue Zaïre-Afrique a repris son ancienne dénomination (Congo-Afrique). La revue de l'université de Gand Afrika Focus qui remplace, depuis 1985, l'AVRUG-Bulletin, est copubliée par la GAP (Gents Afrika Platform) et Afrika Brug. Elle peut être consultée maintenant en version électronique. On signalera l'existence, depuis 1997, d'une nouvelle revue, CongoMeuse, du Centre d'Etude des Littératures 
Belge et Congolaise de Langue Française (CELIBECO), qui a publié les actes de colloques traitant de questions culturelles, certains organisés à Kinshasa.

\section{LES OUVRAGES GÉNÉRAUX ET ENCY- CLOPÉDIQUES}

On trouvera dans la deuxième partie de I'Histoire des sciences en Belgique 18152000 dirigée par R.Halleux et al., un bref aperçu historique des recherches menées par les Belges outre-mer dans les domaines des sciences sociales (Poncelet M., 2001), de la géographie (Nicolaï H., 2001), des sciences de la Terre (Delhal J., 2001) et de la botanique et de la zoologie (Symoens J.-J., 2001). Signalons, dans une collection, qui a l'ambition de couvrir tous les pays du monde, une bibliographie du Zaïre. Elle compte 829 références, avec trois ou quatre lignes de résumé, regroupées en une grosse trentaine de rubriques. Elle a été préparée à la Northwestern University Library (Evanston, Illinois) à partir des collections de la Bibliothèque d'Etudes africaines Melville J. Herkovits. Elle est destinée à des lecteurs anglophones mais elle n'a pu ignorer quelques publications en français (et même quelques-unes en néerlandais). Le contenu est assez hétéroclite. Bien qu'une chronique d'une page concerne en principe la géographie, les géographes belges en sont totalement absents (William D.B., Lesh R.W. et Stamm A.L., 1995).

Un précieux inventaire des recherches africaines en Belgique concerne essentiellement les sciences humaines (Simons E., 1993).

Un livre comptant de nombreuses cartes thématiques a accompagné une exposition présentée au siège de I'UNESCO à Paris, en septembre 2004, puis au Musée de Tervuren sur Nature et Culture en République démocratique du Congo. II insiste sur la biodiversité, sur la nature comme source de matières premières (mines anciennes et mines redécouvertes), d'aliments et de produits pharmaceutiques, sur la diversité culturelle, etc. Oeuvre de membres du personnel scien- tifique du Musée et de scientifiques congolais, il traite aussi quelques points particuliers comme par exemple les solutions à envisager pour lutter contre le braconnage dans les Parcs nationaux (Nature et Culture en R.D.C., 2004).

Des aspects physiques et humains des trois pays qui nous concernent trouvent leur place dans un ouvrage qui reprend les actes d'un colloque sur le Grand Rift Africain, organisé à Bruxelles en septembre 2005 (De Dapper M. \& de Lame D., 2006)

\section{LA CONNAISSANCE CARTOGRA- PHIQUE DU PAYS}

Un bref historique de la représentation cartographique du Congo de 1885 à 1960, comporte une dizaine d'exemples de cartes topographiques et de cartes thématiques (Nicolaï H., 2005). Voici un article sur l'historique du réseau triangulé. Les premiers travaux de triangulation ont été effectués pour la délimitation des frontières (1897-1924). Des canevas régionaux furent établis de 1921 à 1942, par exemple à Kilo-Moto. Ils ont été prolongés vers le parc de la Garamba, dans les territoires administrés sous mandat et dans la zone contiguë du Kivu (cela représente finalement une grande bande méridienne orientale, si on y ajoute les travaux menés par le Comité Spécial du Katanga), enfin dans le Bas-Congo. II y eut par la suite des travaux de raccordement des différents réseaux et des travaux d'ajustement général. Mais ils n'ont pas été terminés. A l'occasion de ces opérations d'ajustement (et de raccord avec le réseau anglais), on notera que l'on avait pu constater que les repères situés entre les lacs Tanganyika et Kivu avaient subi, en une trentaine d'années, un déplacement horizontal relatif de l'ordre du mètre, en relation sans doute avec l'ouverture du fossé tectonique Rien n'a été fait dans toute la partie forestière du nord-ouest et de la cuvette (Meex P., 1997). Les méthodes traditionnelles de triangulation étant devenues désuètes, en collaboration avec des firmes privées, I'IGN (Belgique) a effectué en 2005 une révision de la partie australe du réseau géodésique du Congo en utilisant les 
techniques GPS: 35 nouveaux points ont été implantés, levés et calculés entre Kinshasa et Lubumbashi et, pour la première fois, intégrés dans un système mondial de référence terrestre (ITRS), avec trois stations de référence à Kinshasa, Tshikapa et Lubumbashi. Il a été difficile de comparer les coordonnées mesurées pour ces nouveaux points avec celles des points relevés auparavant car beaucoup de repères ont été détruits et de plus on ignore souvent dans quel système de projection, ces coordonnées avaient été calculées (Van den Herrewegen M., De Doncker F., Voet, P. and Vanden Berghe I., 2006).

Le Musée Royal de l'Afrique Centrale, à Tervuren, publie des cartes générales et des cartes thématiques du Congo, qui ont été mises à jour notamment par le recours aux images satellitaires. On notera une très récente carte administrative et routière au 1/2 500000 qui donne les limites des 26 provinces dont la nouvelle Constitution prévoit bientôt le fonctionnement ainsi que l'état actuel du réseau routier (Africa-Tervuren, 2008), une carte administrative et routière (2008), une carte des ressources minières (2006), une réédition de la carte géologique (2006), une carte de l'utilisation du sol (2008), une carte géologique de l'arc cuprifère (2007), de la ceinture kibarienne du N-E, (2007), des cartes géologiques à plus grande échelle de différentes parties du Katanga, etc. ainsi qu'une carte satellitaire du Kivu sur CD-ROM (2002) et diverses cartes satellitaires.

Exécuté en vue de l'organisation du référendum de 2005 et des élections législatives et présidentielles de 2006, à l'intention de la Commission Electorale Indépendante, un Atlas de l'organisation administrative de la $R D C$ donne à des échelles proches de 1/1 000000 (dans quelques cas, le 1/50 000 ou une échelle plus grande pour des circonscriptions urbaines), pour chaque territoire ou ville, les limites des groupements (subdivisions traditionnelles des secteurs, eux-mêmes subdivisions des territoires), 5397 au total. Certaines délimitations se fondent sur des recherches ou des cartes exécutées au cours des décennies 1950-1960 par le Cemubac. L'atlas comporte également une carte administrative générale, une carte des villes et centres en 2004, une carte des groupes linguistiques, la liste des ethnies par territoire et un index général (de Saint Moulin L., 2005).

\section{LE MILIEU NATUREL}

\section{LA CLIMATOLOGIE}

Deux colloques internationaux ont été organisés à Bruxelles, dont l'un, en 1996, en mémoire du climatologue Franz Bultot (Demarée G., Alexandre J., De Dapper M., 1998 et 2004). II sera rendu compte ci-dessous des communications intéressant l'Afrique centrale.

\section{Les séries chronologiques}

On retrouve dans les publications climatologiques des géographes après 1990 les tendances de la période antérieure avec un accent particulier sur le Katanga. Les données sont fournies par les séries diachroniques d'avant 1960 dont quelques-unes ont pu être prolongées. Ainsi l'analyse statistique des périodes sèches à Lubumbashi, de 1921 à 1989, entreprise pour vérifier s'il existe une petite saison sèche au milieu de la saison des pluies, montre que la persistance de l'absence de pluie n'est que 2 à 3 jours en décembre, janvier et février. La probabilité d'avoir un jour sec de plus par temps de sécheresse est un peu plus élevée en janvier que pendant les deux autres mois mais cela ne suffit pas pour que l'on puisse, à ce moment, parler d'une «petite saison sèche» (Assani A.A., 1993). La variabilité des précipitations est mise en relation avec certains mécanismes de la circulation atmosphérique (oscillation australe) et océanique (El Niño, La Niña) (Assani A.A., 1999). Signalons aussi l'analyse statistique des maxima annuels de pluie journalière à Kolwezi. En 22 ans, on a enregistré 19 pluies atteignant ou 
dépassant $60 \mathrm{~mm}$. Elles se répartissent sur tous les mois de la saison des pluies avec la moitié en octobre et février soit au début et à la fin de cette saison. La lame d'eau journalière la plus élevée qui a été constatée, soit 143,6 mm, a une période de retour estimée à 50 ans (Assani $A$. et Kalombo K, 1995). Une analyse du même type, à Lubumbashi, a utilisé les données de 73 saisons pluvieuses de 1921-22 à 1995-96 (Demarée G. et al., 1998). Un article examine, dans la même région, l'effet des pluies sur l'environnement en fonction de leur intensité horaire, de leur position dans la saison pluvieuse et aussi du degré de fragilisation de cet environnement lié à l'activité humaine (Kalombo K., 2001).

L'utilisation des données de Yangambi a fourni des courbes (IDF) donnant la fréquence des précipitations en fonction de leur intensité et de leur durée (Demarée G.R., 2004, Mohymont B. et Demarée G.R., 2004).

L'évolution récente des précipitations a été étudiée dans le sud-est du Congo et comparée avec celle des pays voisins. Mais les résultats peuvent prêter à controverse. Ainsi pour la seconde moitié du XXe siècle, l'analyse des séries pluviométriques fournies par le réseau de la Gécamines (c'est-à-dire Kolwezi, Likasi et Lubumbashi) n'a pas permis de déceler, contrairement à ce que certains croyaient, un assèchement du climat depuis 1960. On n'a trouvé aucune corrélation entre le volume des pluies et la valeur de l'indice d'oscillation australe. On remarque aussi, ce qui est fréquent dans les régions tropicales où les pluies peuvent être très localisées, que les évolutions, d'une année à l'autre ou d'une décennie à l'autre, peuvent être différentes dans les trois stations. Ainsi, avant 1970, à Kolwezi, la décennie 1950 a été déficitaire et la décennie suivante, excédentaire, alors qu'à Likasi, les deux décennies ont été globalement excédentaires et que Lubumbashi a connu une alternance d'épisodes déficitaires et d'épisodes excédentaires. Par contre, après 1970, les évolutions ont été semblables dans les trois stations avec une phase déficitaire pendant la première moitié de la décennie 1970, une phase excédentaire pendant la seconde moitié et une période déficitaire pendant toute la décennie 1980 (à Kolwezi, uniquement pendant la première moitié de celle- ci) (Assani A., 2001). Cette anomalie pluviométrique négative aurait été cependant de faible intensité comme dans les autres régions de l'Afrique australe et, au Congo, aurait concerné principalement la bordure nord du plateau du Katanga (Kalombo K., 1995, Kalombo K., Erpicum M. et Assani A. A., 1996). Un autre article envisage l'évolution des trente dernières années pour l'ensemble du bassin du Congo (Sanga Ngoie K. et Fukuyama K., 1996).

Une des dernières publications de Franz Bultot cherchait à déterminer si le Rwanda était menacé par une diminution continue des précipitations. Cette étude faite dans le cadre d'un projet de l'AGCD, dont l'initiateur avait été J.-P. Harroy, a analysé un ensemble de 31 séries de totaux pluviométriques annuels et mensuels couvrant, selon les cas, de 30 à 75 années d'observations. Elle conclut que les précipitations n'ont pas connu de diminution progressive depuis le début du siècle. II y a eu des variations cycliques pluriannuelles dont les effets cumulés ont pu atteindre près de $10 \%$ du total pluviométrique moyen. Les variables caractéristiques de la saison sèche (début, fin et durée) n'ont pas subi d'évolution au cours du temps (Bultot F. et Gellens D., 1994).

\section{La définition des saisons et les études} régionales

Une nouvelle classification des régimes pluviométriques du Congo et de l'Angola (à partir des données de 95 stations météorologiques) n'apporte aucune modification essentielle aux classifications habituelles (Moliba Bankanza J.C., 2004). Notons aussi une tentative d'identification des saisons et de leurs caractéristiques «objectives» pour 6 stations représentatives chacune d'une grande région. Une analyse en composantes principales traite les moyennes mensuelles de 9 variables (rayonnement glo- 
bal solaire, bilan de rayonnement total, précipitations, humidité relative, température, évaporation, déficit de saturation, vitesse du vent, insolation) et veut contourner l'absence de consensus sur la définition d'un mois sec ou d'un mois pluvieux. Elle n'apporte cependant pas de grandes différences par rapport à ce que fournit la simple lecture directe de ces variables. Elle conduit aussi à d'étranges regroupements. L'auteur reconnaît ainsi une quatrième saison à Kinshasa qui comprend les mois de novembre à janvier auxquels s'ajoute le mois d'avril qui en est pourtant éloigné. L'absence de consensus sur la définition du mois pluvieux risque ainsi de se transformer en une controverse sur la notion de saison! (Assani A. A., 1994).

Malgré sa position quasi équatoriale, le Rwanda connaît une période sèche de trois mois (juin, juillet, août) et même de quatre mois sur les plateaux orientaux, mais d'un mois seulement sur la crête Congo-Nil. Une décroissance des pluies d'ouest en est, surtout en octobre et en février, traduirait l'action d'une " mousson atlantique» tandis qu'une distribution homogène des pluies serait en relation avec la présence du «front intertropical» (llunga L., Muhire I. et Mbaragijimana C., 2004). Pour le Kivu oriental, l'analyse des saisons sèches a permis de préciser la date à retenir pour les semailles (llunga $\mathrm{L}$. et Mwininyinkondo K. B., 1993). Dans la région de Kigali (Rwanda), une méthode de même type, qui a étudié les caractéristiques du début de la saison des pluies (fréquence mensuelle d'un jour humide sur cinq, un accroissement pluviométrique constant et une faible probabilité d'une séquence sèche de cinq jours), aboutit à proposer le 26 septembre comme date idéale des semailles (llunga L. et Mugiraneza A., 2006).

Les particularités du climat du BasCongo font l'objet d'une nouvelle tentative d'explication. On sait que, quoique située à $5^{\circ}$ sud seulement, cette région a une saison sèche très marquée et très longue, de durée plus grande et d'intensité plus forte près de la côte qu'à l'intérieur, avec des brouillards au niveau du sol surtout dans la région côtière, y compris le
Mayumbe, et un voile de stratus à basse et moyenne altitude qui s'étend jusqu'à 380 km vers l'est, donc un peu au-delà de Kinshasa. Contrairement à ce qui a souvent été dit, le courant froid de Benguela ne serait pas responsable de ces caractères car il n'arriverait pas jusqu'à cette latitude et qu'il est difficile d'imaginer qu'il puisse faire sentir son effet si loin à l'intérieur des terres. Un phénomène d'upwelling (remontée d'eaux froides près de la côte) ne serait pas non plus à prendre en considération. L'explication se trouverait dans l'existence d'une discontinuité, pendant la saison sèche, entre une strate inférieure où souffle un alizé humide et frais à composante ouest (alizé de Sainte-Hélène) et un flux supérieur constitué par un alizé sec venant de l'est (alizé des Mascareignes et alizé issu de l'anticyclone thermique d'Afrique du Sud). Cette discontinuité ne permet pas à la vapeur d'eau de gagner les strates supérieures. Elle disparaît pendant la saison des pluies par retrait de l'alizé des Mascareignes en dessous de $2000 \mathrm{~m}$ ce qui permet le développement vertical des nuages sous l'effet de l'Equateur météorologique. Les pluies seraient alors beaucoup plus intenses que dans d'autres parties du Congo. Le raisonnement s'appuie principalement sur l'analyse des sondages aérologiques effectués à Kinshasa et qui sont repris d'un article de M. Leroux. L'absence de sondages aérologiques pour le Bas-Congo proprement dit affaiblit sans doute le raisonnement. Les auteurs ne parlent pas non plus de la forte amplitude thermique annuelle des stations côtières, la plus élevée du Congo et qui est due essentiellement aux valeurs très basses de la saison sèche, ce qui pourrait impliquer l'effet d'eaux froides (Assani A. A. et Kalombo K., 1997). Notons que, dans un article postérieur, les mêmes auteurs expliquent par la présence de ces eaux froides, l'augmentation habituelles des pluies de la côte vers l'intérieur sauf quand, au moment où l'anticyclone de Sainte Hélène occupe sa position la plus australe, en février-mars, sous l'effet de la proximité de la mousson atlantique, les pluies diminuent d'ouest en est (Kalombo K. et Assani A., 1999-2000). 
Les dates de début et de fin de saison des pluies dans le Bas-Congo présentent une grande variété interannuelle qui a des conséquences importantes pour les activités agricoles. Elles ont été définies dans les régions voisines des plateaux Téké et le plateau des Cataractes au Congo-Brazzaville (Samba G. et al., 19992000), à partir de la notion de «pluie potentiellement utile» $(20 \mathrm{~mm}$ de pluie cumulés sur deux jours non suivis d'une période de sept jours sans pluie pendant vingt jours).

Quelle est la connaissance empirique que les agriculteurs du Bas-Congo ont des mécanismes météorologiques? II apparaît qu'ils sont sensibles aux phénomènes qui annoncent par exemple l'arrivée de la saison des pluies. Cela se traduit dans la façon dont ils organisent les feux de défrichement. Cette sensibilité s'exprime aussi dans les rites associés à la pluie ainsi que dans de nombreux dictons populaires et proverbes qui font référence, parfois avec une certaine justesse, à des événements météorologiques. II y a une terminologie paysanne très poussée des différents types de pluie (Mpasi Ziwa Mambu, 1995).

La paléoclimatologie et les changements de climat

La paléoclimatologie et les changements climatiques futurs sont abordés dans plusieurs publications. Un gros article fait la synthèse de recherches menées sur ces questions, depuis 1980, dans la région des Grands Lacs, plus exactement dans la branche occidentale du Rift africain et dans l'ouest de l'Uganda. Les études stratigraphiques des formations quaternaires, les séquences de géosols et les corrélations établies avec les données isotopiques des fonds océaniques semblent confirmer que les phases chaudes et humides des Tropiques correspondent aux phases chaudes humides des régions tempérées et boréales, donc avec les interglaciaires. La phase de froid maximal il y a 20000 ans et l'épisode froid du Dryas correspondent à une aridification du monde entier avec expansion du Sahara et sécheresse dans les régions tropicales. Les 10000 dernières années auraient connu un grand nombre de cycles de sécheresse dont le dernier serait la sécheresse actuelle du Sahara. II est fâcheux que les auteurs s'écartent de leur analyse des observations scientifiques pour terminer leur article par une réflexion à la Toynbee sur l'effet créateur des épisodes arides: «Droughts rather than Pluvials in the Equatorial Belt of Africa provided Ancient Man with the necessary courage and force of the fittest to cope with this challenge in his daily struggle for life» (Van Overloop E. \& Paepe R., 1998).

Des événements climatiques ont aggravé les effets de la crise politique au Rwanda si l'expression peut encore avoir un sens devant les morts innombrables causées par celle-ci. L'insuffisance des pluies pendant la première partie de la saison des pluies à la fin de 1993 avait réduit fortement les premières récoltes de 1994 (de $27 \%$ au total). Le génocide d'avril et les déplacements de populations, qui ont suivi, ont empêché la deuxième récolte puis les plantations dans les fonds de vallée en saison sèche et enfin les plantations de la première partie de la saison des pluies 94-95 (Gommes R., 1998).

Les sédiments du lac Tanganyika ont enregistré les variations climatiques. Leur étude fait l'objet d'un grand programme Climlake auquel participe activement le Musée royal d'Afrique centrale (voir plus loin le paragraphe Hydrologie et limnologie)

\section{LA GÉOLOGIE}

On ne s'étonnera pas que le Katanga continue à être le champ privilégié de la recherche géologique. Parmi les publications qui intéresseront directement le géographe, signalons d'abord les actes d'un colloque sur la géologie du Katanga et plus spécialement sur ses gisements miniers. Ce colloque s'est tenu à Mons à l'occasion du centenaire des premières observations de Jules Cornet (Colloque international Cornet, 1997). On y trouvera notamment une mise au point très commode sur la stratigraphie de l'arc cuprifère qui tient compte des travaux réalisés entre 1950 et 1980 par le Département Géologique de I'Union Minière puis de la 
Gécamines. Elle propose quelques modifications à la terminologie. Rappelons qu'en raison de l'absence de fossiles caractéristiques, la stratigraphie du Katanguien, dans lequel se trouvent les gisements de cuivre, a été établie essentiellement sur une base lithologique et sédimentologique. Le système Katanguien, bien encadré par les phases ultimes de l'orogenèse kibarienne (1 100 Ma) d'une part et de l'orogenèse katanguienne (620 Ma) d'autre part, est subdivisé en trois supergroupes, soit, du plus ancien au plus récent, le Roan, le Kundelungu inférieur (l'auteur propose de ne plus utiliser le terme Kundelungu pour cet ensemble mais le terme Guba) et le Kundelungu supérieur, dont la base est constituée par le Petit Conglomérat. La plupart des gisements se trouvent dans le groupe des Mines qui fait partie du Roan (groupe R2 que l'auteur propose d'appeler groupe de Kolwezi) (François A., 1997). On trouvera dans le même ouvrage des mises au point sur l'origine des minéralisations et sur la récupération des masses considérables de minerai qui se trouvent dans les anciens rejets d'exploitation, qui résultent des techniques utilisées naguère pour traiter le minerai.

L'article d'A. François, qui vient d'être cité, signale que, sur les 45 mines exploitées au Katanga, 25 étaient épuisées ou inexploitables en profondeur et que, dans les 20 autres, les minerais les plus rentables, donc surtout les minerais peu profonds, avaient déjà été extraits. Une mise au point récente rappelle que, jusqu'en 2003, on a produit 18 millions de tonnes de cuivre-métal, 500000 t de cobalt, 3,6 millions de tonnes de zinc et $280000 \mathrm{t}$ de germanium. II resterait $60 \mathrm{M}$ de $\mathrm{t}$ de cuivre, 5,1 M de t de cobalt, $100000 \mathrm{t}$ de germanium ce qui placerait le Katanga en deuxième position dans le Monde pour les ressources en cuivre et en première position pour le cobalt. Plus que jamais l'industrie minière devrait être la source principale de revenus de la RDC. On sait que la production de cuivre s'est effondrée après 1990 pour passer à moins de 50000 t (30 000 t pour la Gécamines) en 2001. La production reprend. Elle aurait été de 263000 t de cuivre et de 22750 t de cobalt en 2006. On prévoit même $600000 \mathrm{t}$ de cuivre en 2010. Le Congo aurait alors dépassé le pic atteint en 1982 et retrouvé son rang de deuxième producteur mondial pour le cuivre et de premier producteur pour le cobalt. L'article passe en revue les gisements (une soixantaine) et en donne les caractéristiques (les ressources en cuivre, cobalt, argent et zinc, souvent l'historique, la production de 2006 et la société actuelle exploitante), y compris celles de Big Hill, le grand crassier de Lubumbashi où l'on récupère $3500 \mathrm{t}$ de cuivre, $5000 \mathrm{t}$ de cobalt et 15000 t d'oxyde de zinc par an. La production artisanale, illégale ou légale, serait de 150000 t de concentrés de cuivre (50 $000 \mathrm{t}$ de cuivre métal) et de 100000 t de cobalt métal par an. Elle serait assurée par environ 100000 «creuseurs». Après un passage dans de petits fourneaux électriques locaux, les produits sont achetés par des négociants pour être raffinés en partie en Zambie et de là évacués vers la Chine. A noter que l'article a été écrit avant la signature des grands accords entre les gouvernements congolais et chinois. De grands espoirs sont fondés sur l'utilisation de nouvelles techniques (comme la technique SX/EW) qui permettraient d'assurer la transformation totale du minerai dans le pays même (Goossens P., 2007). Sur la problématique des ressources minières, voir aussi l'article de Minani Bihuzo R., 2007.

La région du Copperbelt est une des plus riches provinces métallogéniques du monde. La précipitation des métaux dans les sédiments d'âge néoprotérozoïque a commencé dès le début de la sédimentation et a continué lors de l'enfouissement du bassin. Des minéralisations se sont produites aussi pendant les phases orogéniques et post-orogéniques, phases qui ont connu en outre des migrations de différents types de fluides. II y a donc une grande diversité de processus. Une controverse porte sur leurs importances respectives (Muchez Ph. et Dewaele St., 2006). Un article fait la synthèse des caractéristiques des gisements d'uranium des provinces cuprifères du Katanga et de Zambie. Les roches très métamorphisées de la région des "dômes" 
seraient la source primaire de l'uranium qui s'est reconcentré dans les terrains sédimentaires néoprotérozoïques du système du Katanga pour être mobilisé à nouveau pendant l'orogenèse pan-africaine. Les gisements correspondent ainsi à plusieurs groupes d'âge (notamment 625 et $530 \mathrm{Ma}$ ) (Charlet Ch. et Loris H., 2005). Etant donné que les gisements uranifères émettent des gaz rares, particulièrement du radon, la mesure de l'émission de ce gaz à la surface su sol peut constituer une méthode de prospection si elle est associée à la mesure d'autres anomalies géochimiques de surface. Elle a l'avantage d'être peu coûteuse. Elle a été expérimentée au Katanga (Charlet J.-M. \& Makabu G., 2000).

Si le «Graben de l'Upemba», au Katanga, n'est en fait qu'une dépression morphologique entre les inselbergs des Monts Hakansson et une grande faille au sudest, le plateau du Kundelungu correspondrait à un paléograben anté-Karroo qui aurait conservé les terrains du katanguien (plissés ou tabulaires) (Dumont $\mathrm{P}$. et Hanon M., 1997).

On a exagéré l'importance des ressources minérales des régions bordières du Rift occidental, notamment au KivuManiema. Certes, il y a de nombreux petits gisements dans les terrains précambriens (or, coltan associé à l'étain et au wolfram, chrome, nickel, etc.), et un peu d'hydrocarbures dans les sédiments du fond des fossés, ainsi qu'un peu de méthane (en partie renouvelable) dans le lac Kivu. Mais l'exploitation de la plupart de ces gisements n'est pas économiquement rentable (Tack L. et al., 2006). II est curieux que la présence de minerais de platine dans la région de Lubero, dans le Nord-Kivu n'ait suscité que peu d'intérêt dans la communauté géologique bien que le type de minéralisation auro-platinifère dans lequel on les a trouvés soit très rare (lié à des phénomènes hydrothermaux sans filiation directe avec des roches ultrabasiques comme dans la grande majorité des gisements économiques de platine). Curieux aussi que cette présence n'ait pas intéressé davantage les sociétés minières (Jedwab J., 1999).

\section{LA GÉOMORPHOLOGIE}

L'utilisation du satellite radar ERS-1 SAR apparaît très utile pour les recherches géomorphologiques dans les régions de forêts humides. Des exemples sont donnés pour le Kivu (Runge J., 2001). On trouvera aussi, dans une revue de vulgarisation de la coopération belge au développement, d'autres exemples d'utilisation d'images satellites, mais cette fois surtout dans des régions rendues peu accessibles par la guerre et portant davantage sur des faits humains (Vansteenvoort L., 2001).

Les processus d'érosion et l'évolution des versants

Les processus d'érosion continuent d'être étudiés mais de façon moins systématique que dans les périodes précédentes. Depuis mai 1997, plusieurs glissements de terrain, qui se sont produits à Bukavu et dans ses environs, ont pu être liés à une activité sismique dans la partie sud du bassin du lac Kivu. Des mouvements sismiques ont été enregistrés au Centre de Lwiro et perçus par la population avant le glissement. L'ampleur de ces glissements est favorisée par l'action de l'érosion régressive, qui fragilise les versants, par la présence d'une nappe aquifère et par l'action anthropique (déboisement et construction) (Munyololo Y. et al., 1999). Non loin de là, la ville d'Uvira (150 000 habitants), aménagée sur des terrasses fluviales ou lacustres au nord du lac Tanganyika, au pied de l'escarpement occidental du fossé tectonique, est ravagée régulièrement par des coulées de débris et par l'action érosive des rivières descendant de cet escarpement surtout aux mois de mars et d'avril. Des mesures sont proposées pour protéger les quartiers habités et la voirie (Ilunga L., 2006).

Voici une nouvelle étude sur les cirques d'érosion. Elle concerne ceux qui échancrent les versants des hauts plateaux du Kwango-Kwilu et la ville de Kikwit. Dans le territoire de Gungu, le groupe impressionnant des cirques des sources de la Lukwila, affluent de la Loange (le bord de 
certains d'entre eux pourrait reculer d'une dizaine de mètres en une année), marque incontestablement une rupture d'équilibre car ils déchirent des versants aux pentes convexes. Faut-il expliquer ce déséquilibre, c'est-à-dire cette violente reprise d'érosion par un abaissement du niveau de base de l'ensemble du bassin qui résulterait de la vidange d'un lac qui aurait occupé le centre de la cuvette congolaise? L'hypothèse ne nous paraît pas convaincante étant donné que l'on n'a pas découvert de traces de ce lac dont le niveau aurait dû être bien au-dessus de celui du Pool Malebo. Nous penserions plutôt à l'action de mécanismes locaux qui attaqueraient une topographie héritée d'un système climatique différent, et par conséquent aujourd'hui instable, certains de ces mécanismes pouvant être d'ailleurs d'origine anthropique. Cette dernière origine est bien perceptible en tout cas pour les cirques qui déchirent la ville de Kikwit. Ils n'existaient pas voici une cinquantaine d'années et ont pris parfois des proportions catastrophiques. Nous en constations seulement les premiers déclenchements dans notre propre étude sur le Kwilu au milieu des années 1950 (Salomon J. N., 1998).

L'érosion menace aussi la ville de Kigali (on lira, en fin de ce chapitre, les problèmes d'environnement qu'elle pose à Kinshasa). Or, à Kigali, l'analyse de la répartition des pluies (total annuel $996 \mathrm{~mm}$ : 4 mois secs, $1^{\circ} 1 \mathrm{C}$ d'amplitude annuelle), le calcul du bilan hydrique et les caractéristiques des bassins versants découpant une surface d'aplanissement avec d'épaisses couches d'altérites dérivés de roches granitoïdes indiquent qu'il ne faut pas s'attendre à un fort ruissellement ni par conséquent à une forte érosion des sols. Mais l'extension urbaine a entraîné l'imperméabilisation de surfaces importantes les rendant vulnérables à l'érosion (Ilunga $\mathrm{L}$. et Tshenda A., 2004).

Les collines convexes du plateau de Butare-Ngozi dans le sud du Rwanda et le nord du Burundi montrent de nombreuses traces de cicatrices de décollement et de loupes de glissement qui auraient affecté le manteau de ces collines avant 3000 BP.
Il y aurait eu ensuite une période de stabilité des versants mais la déforestation et l'extension des surfaces agricoles ont provoqué, à l'époque actuelle, une remobilisation de ces mouvements de masse et une érosion par ravines (Moeyersons J. et al., 2004).

L'article de J.N. Salomon (1998) cité plus haut a étudié les dépôts d'une terrasse du Kwilu à Kikwit. Ils contiennent des galets de grosses dimensions dont l'origine serait lointaine. II faut donc songer à un type d'écoulement différent de l'écoulement actuel, vraisemblablement au cours du dernier interpluvial africain (20 000 à 12000 B.P.) pendant lequel le mode de fonctionnement de la rivière aurait été plus spasmodique (avec cependant une saison des pluies capable de fournir de gros écoulements). Au Katanga, une terrasse de la Kavubu, à une douzaine de kilomètres au sud-est de Lubumbashi, a pu être étudiée sur plus de $100 \mathrm{~m}$ de longueur, dans la tranchée d'un canal d'irrigation. Elle est associée à un seuil rocheux attaqué par l'érosion régressive. Une très mince couche d'alluvions caillouteuses a été déposée pendant une phase climatique humide. Une phase sèche ultérieure a entraîné l'envahissement du lit par des grenailles latéritiques provenant de l'érosion du versant. L'action des termites a contribué à transformer les sédiments en une stone-line ondulée (Dibanga B. et Alexandre J., 1996).

\section{La charge des rivières}

La charge solide du fleuve Congo est considérée généralement comme peu importante. Cependant, dans le canal de dérivation de $9 \mathrm{~km}$ de longueur qui amène une partie des eaux du fleuve à l'usine d'Inga, la charge solide peut atteindre $50 \mathrm{mg} / \mathrm{l}$. Après 16 ans de fonctionnement des deux bassins successifs aménagés pour la décantation des eaux avant l'arrivée à l'usine, le premier, pour les particules grossières, et le second, pour les «vases», le premier est fortement ensablé et ne joue plus son rôle et le second est menacé d'ensablement (Bizimana B. et al., 1993). 


\section{L'érosion des sols agricoles}

L'érosion des sols, dans les régions accidentées de l'Afrique tropicale, a préoccupé depuis longtemps les autorités et les agronomes. Une bonne partie du numéro 185 des Cahiers d'Outre-Mer (1994) lui est consacrée. Ce qu'on peut lire, dans ce numéro et dans la bibliographie des dix dernières années, ne renouvelle pas vraiment la question. Et pourtant celle-ci préoccupe aussi les paysans parce qu'elle entraîne une incertitude sur l'avenir de leur patrimoine foncier et suscite leur interrogation sur la rentabilité des aménagements anti-érosifs qu'on leur propose ou qu'on leur impose (Charlery de la Masselière C., 1994). Sur les fortes pentes, l'érosion peut emporter $100 \mathrm{t}$ de terres à l'ha chaque année (König D., 1994).

Dans ce pays très peuplé qu'est le Rwanda, les pentes supérieures à $30 \%$ concernent plus de la moitié des terres cultivées. Les méthodes utilisées par les autorités belges depuis 1938 pour lutter contre l'érosion ont été abandonnées avec l'accès à l'indépendance car elles ont été considérées comme l'expression d'une contrainte coloniale. Elles ont cependant été reprises et généralisées après 1973 pour s'interrompre en 1990 avec le début de la guerre civile. II faut mettre en balance les avantages et les inconvénients des principales méthodes utilisées: fossés anti-érosifs, rangées d'herbes (le procédé le plus répandu) bordant ou non des fossés, terrasses. Aujourd'hui on préconise les terrasses, sans mur et sans fossé mais avec une contre-pente. Le procédé rencontre peu de succès. II se heurte à des difficultés socio-culturelles et exigerait un remodelage foncier (Sibamana C. et Moeyersons J., 1996). Sur la valeur de la pente qu'on peut considérer comme seuil pour l'érosion par ravinement, voir aussi Moeyersons (2003). Un agronome préconise des méthodes biologiques comme la culture en couloirs entre des haies de légumineuses (voir article de König, cité plus haut, 1994). L'homme peut aggraver l'érosion ou la réduire par le choix des systèmes culturaux. Les champs de bananiers jouent un grand rôle dans une ges- tion conservatrice de l'eau et du sol (Rishirumuhirwa T., 1994). Une étude faite dans la région de Ruhengeri, où les paysans sont loin de détenir la maîtrise de leurs eaux, montre les dégâts occasionnés aux cultures et aux infrastructures. Un autre problème se pose aussi, celui de l'approvisionnement en eau potable pour les familles vivant sur la partie haute des collines (Nzamwita D., 1993). Même problème d'approvisionnement pour les habitants des planèzes au pied des volcans malgré l'abondance des pluies mais en raison de la rareté des sources et des eaux courantes (Kabanda T., 1993).

\section{Quelques données pédologiques}

Les procédés utilisés jusqu'à présent pour reconstituer la fertilité des sols dégradés du Rwanda n'ont pas donné de résultats satisfaisants. On propose d'utiliser un engrais qui serait fait d'un mélange de cendres volcaniques et de bouse de vache (Stache N. et Wirthmann A., 1998)

Dans le Bas-Congo, les sols sont très altérés avec une fraction colloïdale où prédominent la kaolinite et les sesquioxydes. Sauf là où la densité de la population est faible et les jachères peuvent donc être de longue durée, l'agriculture traditionnelle ne permet pas de conserver la matière organique et la capacité d'échanges ioniques des profils supérieurs. L'érosion des sols en outre est forte au début de la saison des pluies (Baert G. et Van Ranst E., 1998).

Sur l'utilisation des données pédologiques pour déterminer la capacité des terroirs des régions orientales et du Rwanda, voir les références fournies à la page 320 de cette chronique.

Les formations superficielles et les cuirasses

On attendait depuis longtemps, du grand spécialiste des formations latéritiques et de leurs surfaces d'aplanissement corrélatives au Katanga, un livre de synthèse sur les résultats de ses recherches. Le voilà publié, d'une grande richesse et sous un format commode. Après une présentation 
de la région et un lexique des différents horizons d'un profil latéritique, le livre étudie les stone-lines ferrugineuses et les cuirasses (y compris les carapaces), décrit les processus de formation des enduits ferrugineux, des nodulations et concrétions et des cuirasses proprement dites ainsi que l'action des termites et expose ensuite les procédés de datation des cuirasses et des dépôts corrélatifs utilisés pour reconstituer l'histoire géomorphologique du Haut-Katanga austral. La genèse des cuirasses et des formations ferrugineuses est décryptée à partir de l'analyse d'échantillons prélevés d'une part sur un substratum précambrien de siltites à aspect schisteux et d'autre part sur un substratum sableux cénozoïque (Alexandre J., 2002).

Si l'on n'observe pratiquement pas de formations bauxitiques au Katanga, il en existe par contre, dans la partie orientale du Mayumbe. Elles se sont développées à partir du manteau d'altération de roches doléritiques précambriennes. Voici cinquante ans, on envisageait même de les faire traiter dans une usine d'aluminium qui aurait trouvé sa place dans les projets industriels liés au barrage d'Inga. Ces profils bauxitiques correspondent à deux surfaces d'aplanissement datées miTertiaire et Pliocène-début Pléistocène (Kaseba M. K. et al., 1997).

Les archéologues éprouvent de grosses difficultés à définir la position stratigraphique des gisements paléolithiques qu'ils trouvent dans les stone-lines. Des études faites dans le sud du Congo-Brazzaville, le Gabon, le sud du Cameroun mais aussi au Katanga ont trouvé de nombreuses stonelines contenant des outils paléolithiques attribués au Sangoen (70 000- 40000 BP). Ces stone-lines correspondraient à une surface d'érosion avec pavage datant du Maluekien qui aurait été recouverte par des matériaux meubles remontés par les termites et par des épandages latéraux au bas des versants (Schwartz D., 1996).

\section{Changements climatiques et géomorpho- logie}

Une grosse communication à un colloque organisé par l'Académie Royale des
Sciences d'Outre-Mer sur les changements climatiques et la géomorphologie dans les environnements tropicaux, décrit l'état de nos connaissances sur cette question pour le Quaternaire de l'Afrique centrale. II s'appuie sur les éléments énumérés ci-après.

- Les données fournies par les glaciers et les traces glaciaires. Le Ruwenzori a fourni jusqu'à présent peu d'informations. Le Mont Kenya en a donné davantage. Trois glaciations au moins y ont été identifiées et datées. Elles correspondent aux dernières glaciations des régions extra-tropicales. Ces périodes froides seraient aussi des périodes plus sèches.

- Les données fournies par le milieu lacustre. Les rivages des lacs sans exutoire du Rift oriental conservent des traces de niveaux plus élevés mais pas les lacs avec exutoire, comme le Victoria et le Tanganyika. Pour ce dernier, l'analyse des sédiments loin des côtes apparaît prometteuse.

- Les données de la morphologie fluviale. Les nappes alluviales sont associées à des épisodes secs ou à des épisodes humides, les phases d'érosion à des climats intermédiaires proches de l'actuel.

- Les données de la morphologie des versants. Les phases sèches donnent des glacis d'érosion, des épandages sédimentaires, le pavage résiduel des stonelines et le remblaiement des ravins creusés pendant les phases humides.

- Les données fournies par le milieu karstique, c'est-à-dire les concrétions carbonatées dont certaines sont datées de plus de 150000 ans.

- Les données fournies par les milieux côtiers, c'est-à-dire les sondages de sédiments proches des rivages.

- Les formes d'origine éolienne. On les rencontre par exemple dans des régions recevant aujourd'hui de 1000 à 1400 mm de pluie (plateau du Katanga et de la Zambie voisine). Elles sont encore mal 
datées. Les formes révélées par les images Landsat sous les forêts de la région de Lusambo sont sans doute très anciennes (antérieures au Quaternaire?) et continuent à poser des problèmes d'interprétation (Alexandre, J., Aloni K., De Dapper M., 1994).

\section{LES PALÉOENVIRONNEMENTS}

Les stalagmites des grottes du Mont Hoyo apportent des précisions sur les paléoenvironnements. II y a corrélation entre la formation de trois niveaux de planchers stalagmitiques de calcite et l'existence de forêts denses, entre la formation de concrétions d'aragonite et une phase d'assèchement propice à une végétation de savane arbustive, entre le dépôt de cailloutis concrétionnés par du gypse et un climat froid de type africomontagnard. La température aurait été de $2^{\circ}$ supérieure à l'actuelle $\left(23^{\circ} \mathrm{C}\right)$ pendant l'optimum holocène et de $4^{\circ}$ inférieure lors de l'hypothermal du Pléistocène (Michel R., 1998).

L'analyse palynologique des tourbières de l'est du Congo et du Rwanda a fourni de nombreuses données sur les paléoenvironnements holocènes. Le sondage d'une série sédimentaire de 4,8 m dans un marais de montagne à 2200 m d'altitude dans le Parc National Kahuzi-Bienga, à l'ouest du lac Kivu, a reconnu ainsi un épisode forestier chaud et humide vers $6000 \mathrm{BP}$, une péjoration climatique vers $4000 \mathrm{BP}$, une recolonisation forestière après cette phase et des effets très tardifs de l'anthropisation (avec expansion des Hagenia dans les clairières) à partir de 360 BP (à moins qu'il ne s'agisse dans ce cas d'un effet du Petit Age Glaciaire) (Moscol-Oliveira M. et Roche R., 1997). Une étude abondamment illustrée sur une séquence de 2,2 $\mathrm{m}$ du même marais a été publiée dix ans plus tard. Elle concerne l'évolution qui s'est produite au cours des deux derniers millénaires. Il y a deux mille ans, extension de la forêt ombrophile entre 1600 et 2600 m d'altitude. Vers 500 A.D., pulsation climatique froide avec progression de Hagenia. Au IXe siècle, régression de ce dernier de faible ampleur et qui serait surtout d'origine anthropique. Regain forestier après le XIIe siècle. A partir du XVIIe siècle, secondarisation définitive de la forêt primaire. Sur les rives du lac, cette secondarisation, qui va jusqu'à la savanisation, serait liée à l'installation de populations plus actives dans le domaine agricole que les précédentes (Kabonyi Nzanbandora C., 2007). L'analyse palynologique d'une tourbière rwandaise, au pied des champs de lave du volcan Muhabura a confirmé les résultats d'études antérieures: de 12000 à 7000 BP, passage progressif du climat froid du Tardiglaciaire à un stade plus chaud et plus humide avec encore une oscillation froide vers 9000, un optimum vers $6000 \mathrm{BP}$ avec expansion maximum de la forêt dense montagnarde, un pic d'aridité vers 4000 BP puis une évolution vers un climat à nouveau humide semblable à l'actuel (depuis 2000 BP) avec un bref épisode sec vers 500 A.D. C'est surtout à partir du $X e$ siècle que les activités humaines se marquent par des effets irréversibles sur le milieu (Roche E. et Ntaganda Ch., 1998). Résultats identiques dans un autre article de l'auteur principal des deux articles précédents (Roche E., 1998). Mêmes conclusions dans des articles d'auteurs français ou allemands. Plusieurs étudient l'évolution de la forêt ombrophile de la Kibira (40 000 ha, parc national du Burundi, aux limites du Rwanda sur la crête Congo-Nil) (Peyrot B., 1997) ou celle des paysages végétaux du sud du Rwanda à partir du dernier maximum glaciaire, c'est-à-dire à un moment où cette région de la crête Congo-Nil était, avec le Cameroun et les reliefs du Libéria, un refuge de la forêt équatoriale. La conclusion de l'auteur n'est pas surprenante: les paysages actuels du Rwanda sont des Kulturlandschafte (Kersting P., 2006). D'autres études élargissent la question à l'évolution de la végétation de l'ensemble de l'Afrique centrale (Bonnefille R., Schwartz D. et GDR Ecofit, 2002). Les données phytodynamiques confirment les actions anthropiques sur le milieu montagnard du graben centrafricain (Habiyaremye F. X. et Roche E., 2003). II a fallu attendre l'avènement de la technique du fer dans le sud du Rwanda pour que l'ef- 
fet anthropique sur l'environnement se manifeste dans les analyses palynologiques, c'est-à-dire les premiers siècles de l'ère chrétienne. Cet effet s'accentue à partir du VIIIe siècle (Age du Fer récent). L'arrivée de nouvelles populations au $X^{e}$ siècle et le développement de l'agriculture et de l'élevage ont entraîné des changements importants. II faut tenir compte aussi des deux petites phases climatiques sèches, dont il a été parlé plus haut, au VIe et au XVIe siècle, qui ont accéléré la dégradation du couvert forestier ou de la savane boisée (Roche E., 1996). L'étude géomorphologique de sites choisis dans la grande forêt de l'est du Congo confirme sa dégradation dans les parties qui avaient été des zonesrefuges pendant la période glaciaire (Runge J., 1996)

La vitesse d'accumulation de la tourbe et du carbone a été mesurée dans les tourbières de l'Akanyaru du Rwanda et du Burundi. Pendant la dernière période glaciaire, il n'y aurait eu qu'une faible accumulation de tourbe car le climat était plutôt sec. A l'époque actuelle, les interventions humaines ont plus d'effet sur la vitesse d'accumulation que les variations climatiques (Pajuen H., 1998).

Les phénomènes géomorphologiques dont le creep sont étudiés dans leurs relations avec les conditions environnementales de la fin du Pléistocène et de l'Holocène dans la région de Butare (Rwanda) (Moeyersons J., 2001).

Des phytolithes ont été recueillis et analysés dans trois sites archéologiques de la forêt de l'Ituri. Les plus anciens, datés de 19000 à 10000 B.P. indiquent que la région nord-est du bassin congolais était couverte de forêts à la fin du Pléistocène quand elle a été occupée par l'homme. Depuis lors, l'occupation humaine semble avoir été continue (Mercader J. et al., 2000).

\section{L'HYDROLOGIE ET LA LIMNOLOGIE}

Un grave problème se pose aux chercheurs qui s'occupent de l'hydrologie du bassin congolais. La situation du réseau hydrologique actuel, en ce qui concerne l'observation des niveaux, est inférieure à ce qu'elle était avant la première guerre mondiale. C'est pire encore pour l'observation des débits. Depuis 30 ans (la communication a été présentée en 1996), il n'y a plus d'observations régulières sauf pour le bief maritime. On connaît ainsi très mal la valeur des crues maximales du fleuve Congo en décembre 1961 et mai 1962. De plus les archives des anciennes observations sont dans un état déplorable. II faudrait sauver ces données et améliorer la situation par un effort international, sous le patronage de grands organismes (Charlier J., 1998).

Le lac Tanganyika est étudié dans des projets internationaux qui comportent notamment une comparaison avec le lac Baïkal. Citons d'abord deux articles qui auraient pu s'inscrire dans le paragraphe "Climatologie» puisqu'ils concernent les effets des variations du climat ou des changements climatiques sur le fonctionnement du lac. On a pu mettre en corrélation le brassage des eaux avec les variations du Niño. La tendance à la diminution de la force du vent favorise la stratification des eaux. En effet les vents du sud-est entraînent un upwelling dans la partie méridionale. Une diminution d'intensité de ces upwellings provoque une diminution des poissons cupléides aux deux extrémités du lac. II y aurait ainsi des variations cycliques (période de 3 à 7 ans) dans l'abondance des poissons pélagiques. La tendance au réchauffement récent en Afrique de l'Est se traduit par une augmentation de 0,7 à $0,9^{\circ} \mathrm{C}$ en 27 ans pour la température de l'air audessus du lac. Un grand projet de la politique scientifique belge va recueillir les signaux paléoclimatiques enregistrés dans les sédiments (Plisnier P.-D., 1998). Les relations entre les variations du Niño et le niveau du lac ont été étudiées par Bergonzini L. et Richard Y. (2004). On a établi un modèle des oscillations de la thermocline induites par le vent (Naithani J. et al., 2004). La fluctuation du niveau et le déversement des eaux à l'exutoire sont évidemment liés aux faits climatiques. L'exutoire ne fonctionnait pas entre 1846 et 1878, lors de l'arrivée des premiers explorateurs arabes et européens. En 1878 , le niveau était à 783,6 m, l'article 
n'expliquant pourquoi l'exutoire de la Lukuga ne fonctionnait pas alors que les niveaux ultérieurs seront plus bas: $772,5 \mathrm{~m}$ en 1894, 776,82 m en 1964 (maximum récent), $773 \mathrm{~m}$ en 1994. Les dernières fluctuations sont semblables à celles des lacs Victoria, Kyoga, Albert et Malawi, ce qui confirme que le régime du lac dépend principalement des conditions météorologiques de l'Afrique orientale où s'étend la plus grande partie de son bassin. II y aurait des cycles de 5, 9 et 10-11 ans dans le bilan hydrique. Les estimations du débit à l'exutoire ont été faites depuis 1932. Le module interannuel est de $200 \mathrm{~m}^{3} / \mathrm{sec}$ (le deuxième article donne $380 \mathrm{~m}^{3}$ pour la période 1932-1995), avec un débit maximum en avril-mai et un minimum en octobre-novembre. Depuis 1965, il y aurait tendance à la hausse des écoulements. II suffirait cependant que la pluviosité baisse de $9 \%$ (11\% par rapport aux précipitations moyennes de $1010 \mathrm{~mm} / \mathrm{an}$ pour la période 1932-1980, dans le deuxième article) pour que l'exutoire cesse de fonctionner (Bergonzini L., 1995). Dans un ouvrage important, l'auteur de ces deux articles a élargi ces questions en traitant également le cas des lacs Kivu, Rukwa et Nyassa. A partir d'une banque de données rassemblant et compilant des données éparses, il calcule le bilan hydrique de chaque lac par une équation qui met en relation les échelles mensuelles, annuelles ou pluriannuelles, les variations de stock, les précipitations sur le lac même, les écoulements provenant du bassin-versant, les pertes par évaporation, les sorties par l'exutoire. II donne les bilans mensuels et annuels et les variations interannuelles dont il recherche les causes. II analyse la sensibilité des niveaux lacustres à la variabilité des précipitations et reconstitue les niveaux depuis le dernier maximum glaciaire (18 000 ans B.P.). II estime enfin les paléoprécipitations sur l'Afrique de l'Est (Bergonzini L., 1998). Pour le lac Tanganyika, une thèse de doctorat défendue récemment à Wageningen apporte quelques précisions complémentaires sur les points suivants: la distribution verticale et horizontale du phytoplancton, l'effet des mouvements de l'eau induits par le vent sur les nutriments et les structures de la communauté planctonique, sur la pro- duction de chlorophylle et sur la production primaire, les sources externes de nutriments (la Ruzizi apporte près du double de ce qu'apportent ensemble la Malagarasi et la Lufubu). En utilisant les sources disponibles jusqu'à présent, on n'a pas observé de corrélation entre une hausse tendancielle de la température de surface et une fluctuation des captures totales (Langenberg V. T., 2008). Le lac Tanganyika et le lac Malawi représentent respectivement 16 et $8 \%$ des réserves mondiales d'eau libre de surface. Ils connaissent une eutrophisation progressive d'origine anthropique surtout dans les baies proches des grandes villes. Une grosse étude essentiellement géochimique des structures thermiques et chimiques de leurs eaux et de l'enregistrement sédimentaire superficiel de leurs fonds, est précédée d'une présentation générale, en une trentaine de pages, du cadre général de la branche occidentale du rift africain au point de vue géologique, climatique, géomorphologique, hydrologique et biogéographique (Branchu P., 2001).

A partir de données du Congo-Brazzavile, une comparaison de deux systèmes hydrologiques différents peut être appliquée aux territoires voisins du CongoKinshasa. Elle oppose la Cuvette et les Plateaux Téké. Dans la Cuvette, le coefficient d'écoulement est de 20-30\%; dans les plateaux Téké, 45-60\%. La variabilité saisonnière des débits est de 2,5-5,5 pour le premier cas, 1,1-1,5 pour le second. L'aquifère gréseux des plateaux a donc un effet régulateur. II fournit des eaux «blanches», peu minéralisées mais hypersiliceuses. Dans la cuvette, les terrains sont moins perméables, l'évaporation est forte dans les zones inondables, les eaux, très chargées en acides forestiers fulviques et humiques, sont «noires» (Laraque A. et Olivy J.-C., 1998).

\section{LA BIOGÉOGRAPHIE}

\section{Le couvert végétal}

Voici d'abord quelques précisions sur la végétation du Katanga. Les îlots de forêt dense sèche que l'on rencontre dans le sud du Katanga correspondent vraisemblablement à un stade de reconquête 
après le dernier épisode aride de 30004000 B.P. pendant lequel s'était développée le forêt claire zambézienne. Ces îlots sont aujourd'hui très vulnérables à l'action anthropique (Dikumbwa N. et Mbenza M., 1999).

La flore associée aux gisements de cuivre ne cesse pas de susciter l'intérêt. Les progrès accomplis de 1977 à 1994, c'està-dire depuis les travaux de $P$. Duvigneaud, ont fait l'objet d'une synthèse qui comporte une grosse bibliographie de 76 références sur la phytogénétique, la phytochimie ainsi que sur les conséquences de la pollution minière (Malaisse F., 1994). Cette flore présente une grande diversité. De nombreuses familles de végétaux évolués sont représentées (Leteinturier B. et Malaisse F., 1999). Avec leurs corolles chatoyantes, leur aspect de «bouquets tout faits» et la longue durée de leur floraison, les Buchnera signalent de façon très visible les gisements cuprocobaltifères (Malaisse F., Baker A. J. M et Leteinturier B., 1997).

Cette végétation a évidemment disparu de la plupart des sites exploités. Mais l'exploitation minière a pollué de nombreux sites situés en dehors des excavations elles-mêmes: sites de lavage, ballasts de voies ferrées et accotements de routes rechargés avec des minerais, accumulations de déblais, etc. La diffusion de la pollution a encore augmenté avec la privatisation des exploitations. II devient donc nécessaire d'envisager une politique de réhabilitation des sites pollués par les métaux lourds. Des techniques de phytoremédiation pourraient être appliquées en utilisant des plantes hyperaccumulantes qui seraient incinérées après récolte. Mais les études et l'expérimentation de ces techniques n'en sont encore qu'à leurs débuts (Leteinturier B. et Malaisse F., 1994).

Le Mayumbe, bien que son climat soit marginal pour la forêt dense, fournissait, en 1969, $70 \%$ du volume national de la production forestière congolaise avec $1,4 \%$ seulement de la superficie forestière totale du pays. Aussi, dès avant 1960, des programmes de reboisement (du Limba pour $78 \%$ ) ont été organisés par la technique des layons combinés à des champs de bananiers. Après une quarantaine d'années, les résultats obtenus dans un espace situé à $28 \mathrm{~km}$ au nord de Boma, permettent de tirer des enseignements pour une politique nouvelle de reboisement. Le Limba a donné de bons résultats. Le Bilinga, expérimenté lui aussi, serait peut-être mieux à sa place dans le climat plus humide de la cuvette (Pendje G., 1993).

\section{Le déboisement}

Les besoins en terres agricoles, en terres à bâtir et en produits divers entraînent un recul du couvert végétal qui est étudié de différentes manières. La comparaison de photos aériennes des années 50-60 et des années 80 essaie de mesurer le phénomène au Congo-Brazzzaville dans la région du Pool. Elle constate la disparition quasi-totale des forêts-galeries (Samba Kimbata M. J., 1995). Pour une superficie de 1243 km² autour de Kinshasa, c'est la comparaison, après numérisation, d'un document cartographique de 1960 et de deux images satellitaires de 1982 et 1987, qui est utilisée. On a identifié chaque fois 5 unités: zone urbaine, forêt, mosaïque forêt-savane, savane et steppe, végétation aquatique (et marais). En 27 ans, rapportée à la superficie totale, l'évolution a été de $+6 \%$ pour la zone urbaine, - $31 \%$ pour les forêts, $+23 \%$ pour la mosaïque forêt-savane, $+3 \%$ pour les savanes et les steppes (y compris les espaces cultivés enclavés) et de - $1 \%$ pour la végétation aquatique et les marais (Tshibangu K. W. T., Engels P. et Malaisse F., 1997). Les besoins en bois de feu interviennent de façon appréciable dans la réduction de la végétation ligneuse. On estime que la consommation annuelle moyenne par personne à Kinshasa est, en 1990, de 160 $\mathrm{kg}$ de bois sec. Une enquête a été menée sur les principaux axes d'accès à la ville (3 axes routiers, le chemin de fer, le fleuve). $77 \%$ du bois viennent du Bas-Congo et $18 \%$ du Bandundu (Tshibangu K. et Malaisse F., 1995).

Sur la dorsale Congo-Nil, au Burundi, la forêt de la Kiribu ne représente plus que $10 \%$ d'une ancienne forêt ombrophile. Le recul est dû aux activités agro-pastorales 
mais a peut-être été facilité par une fluctuation paléoécologique (Peyrot B., 1997). Une enquête menée pendant un an dans des ménages sur huit sites différents du Rwanda montre qu'en 1992, $98 \%$ des ménages utilisent des combustibles traditionnels (bois, charbon de bois, déchets de récoltes, un petit peu de tourbe). Le citadin, qui utilise du charbon de bois, consomme trois fois plus d'équivalent-bois qu'un rural. En 1992, consommation totale de bois: 5,658 millions de $\mathrm{m}^{3}$. II aurait fallu presque quadrupler la superficie des bois de 1992 si on avait voulu assurer la consommation de l'an 2000 en maintenant la valeur totale de la biomasse (Karenzi P. E., 1994).

Un mémoire d'un géographe de l'Université de Varsovie étudie de façon générale la déforestation en Afrique centrale. II s'efforce de délimiter l'extension des forêts denses humides avant que ne s'exerçât l'action anthropique (plus exactement celle de l'agriculteur). Comme l'auteur estime que le climat peut être considéré comme à peu près stable depuis deux mille ans, et plus particulièrement dans toute la partie nord du Congo, la réduction de la forêt est due essentiellement à l'homme. En tenant compte des exigences climatiques de la forêt dense humide, il en trace la limite d'il y a deux mille ans et établit la chronologie des épisodes historiques qui se sont marqués par le recul de la forêt. Celui-ci est lié principalement à la culture sur brûlis qui a fait disparaître les formations végétales intermédiaires entre les forêts denses humides et les végétations à caractère xérophile, mettant ainsi en contact direct la forêt humide et la savane. Le recul aurait été de 150 à $200 \mathrm{~km}$ depuis 2000 ans. Les épisodes principaux seraient donc a) l'introduction de l'agriculture par l'arrivée des populations bantoues, b) l'invasion des peuples soudanais au nord, c) à partir du XVIe siècle, l'introduction des plantes américaines, $d$ ) le développement de la métallurgie du cuivre au Katanga, e) les effets de la colonisation puis de l'explosion démographique du XXe siècle. En comparant les photos aériennes des années 50 et les documents de télédétection actuels, l'au- teur dresse une carte, présentée en horstexte, de l'évolution de la limite de la grande forêt pour la partie nord du Congo. On pourra la comparer avec celle des limites forêt-savane publiée en 1964 par Peeters L. dans la Revue Belge de Géographie, qui couvrait la même portion de territoire (avec en plus la partie extrême-orientale). En extrapolant les données de sa carte, l'auteur estime à 10,1\% la diminution des surfaces forestières pour l'ensemble du Congo de 1955 à 1989, soit $0,3 \%$ par an. Elle concerne essentiellement des espaces à l'intérieur même de la forêt, le long des voies de communication et autour des agglomérations. Pour l'auteur, la dégradation de la forêt au Congo ne prend pas encore des proportions catastrophiques et pourrait être contenue si l'on remplaçait l'agriculture sur brûlis par de nouvelles méthodes (Giralowski J., 2002).

Deux symposiums ont étudié les problèmes d'exploitation durable des forêts tropicales avec quelques exemples pris en Afrique centrale (De Dapper. M., 2004 et 2005). Une communication, après avoir décrit le complexe forestier congolais et analysé les causes de la destruction de la forêt, énumère les initiatives officielles en faveur de sa gestion durable (Koyo J.P., 2005). Une réserve de biosphère, dans un projet soutenu par l'Unesco et la Commission européenne, a été organisée à $L u k i$, à 60 km de Boma, dans une région dont l'exploitation est soumise, non seulement, aux pressions venant de la ville de Boma, mais aussi, depuis la construction du pont de Maradi, à celles émanant de Matadi et de Kinshasa. (Mankoto S. et Maldague M., 2005).

La question générale des changements à court et moyen terme du couvert végétal en Afrique tropicale est traitée aujourd'hui dans de grands programmes internationaux. Celui, dont il est question ici, utilise les images satellitaires. II a détecté les points névralgiques (hot spots) pour lesquels les risques de changements importants sont les plus grands. Pour 15 d'entre eux répartis sur toute l'Afrique, il a établi des modèles spatiaux spécifiques qui permettent des simulations tenant compte des processus identifiés. Mais l'article 
essentiellement méthodologique ne dit pas quels sont ceux qui ont été retenus au Congo (Lambin E., 1998). Une carte des grands types de formation végétale a été établie pour la partie nord-ouest du Congo à partir d'images satellitaires recourant à plusieurs senseurs. Une succession chronologique de cartes de ce type devrait permettre de suivre les changements de végétation (Mayaux P., De Grandi G. et Malingreau J.-P., 2000). Les mêmes auteurs présentent une carte générale de l'Afrique centrale réalisée selon les mêmes méthodes. Les images optiques permettent de distinguer les forêts de terre ferme des formations secondaires et des savanes. Les images radar fournissent des informations sur les milieux humides (forêts et savanes inondées) et les plantations industrielles (Mayaux P. et Malingreau J.-P., 2000). Un autre programme concerne la dégradation de l'environnement dans les espaces péri-urbains. L'article qui le présente énumère aussi les questions posées à cet effet aux habitants des périphéries de Kinshasa et de Lubumbashi (Trefon T. et Cogels S. 2005).

\section{Les ressources du milieu végétal}

Nous voilà abondamment informés sur les ressources, que la forêt claire (Miombo) peut fournir à ses habitants, grâce à une étude ethnobiologique qui concerne surtout le pays bemba, dans le sud du Katanga. Après une description générale du milieu, elle présente les produits sauvages comestibles et les techniques de leur récolte et de leur préparation. Elle estime aussi leur valeur nutritionnelle. Sont envisagés ainsi les champignons, les plantes sauvages, les miels, les grands mammifères, les rongeurs, les oiseaux, les poissons, les reptiles, les chenilles, les termites et autres insectes, les boissons, le sel. L'étude passe en revue également les plantes de l'agriculture traditionnelle, estime l'impact environnemental de la période coloniale, discute la question de la savanisation et du reboisement et propose une gestion de type écologique du territoire (Malaisse F., 1997). Un article analyse la composition chimique et mesure la valeur énergétique de différents produits de récolte et de chasse du même milieu: reptiles, insectes (dont 17 espèces de chenilles), poissons (12 espèces), crustacés (2 espèces), mollusques (2 espèces) (Malaisse F. et Parent G., 1997). Les champignons comestibles font l'objet d'une étude spécifique pour un territoire voisin (la province du Copperbelt en Zambie): liste des champignons, teneur en protéines, en acides gras, en minéraux, techniques de récolte. Les champignons sont une source de revenus non négligeable et une source alimentaire particulièrement bienvenue avant la soudure des récoltes agricoles. Ces ressources sont cependant menacées par la déforestation (Bourdeaux Q. et al., 2003).

F. Malaisse dresse aussi, pour l'ensemble de l'Afrique tropicale, le tableau général des ressources alimentaires non conventionnelles: consommation de Cyanobactéries, mycophagie, consommation de Gymnospermes et de Pinophytes, de Magniophytes, consommation d'insectes (coléoptères, termitophagie, campéophagie, acridophagie, etc.). Ces ressources, qui sont d'une grande diversité, permettent de pallier les situations alimentaires critiques saisonnières (Malaisse F., 2004). Les chenilles sont très recherchées pour leur valeur alimentaire par de nombreux groupes africains, mais pas par tous. Une carte d'Afrique tropicale donne la répartition des groupes campéophages, c'est-àdire consommateurs de chenilles, avec le nombre d'espèces consommées dans chacun d'entre eux. Les données ont été recueillies auprès de 98 groupes ethnolinguistiques. La composition chimique (acides aminés, lipide, vitamines) est aussi fournie pour les différentes espèces (Malaisse F., 2002).

Dans les savanes soudano-guinéennes, les chasseurs ou les cultivateurs, quand ils sont loin du village, se désaltèrent avec la sève contenue dans une liane ligneuse, Cissus Populnea. Cette sève est potable mais avec une teneur élevée en potassium. On pourrait envisager son exploitation (Ambe G.A., Delcarte E. et Malaisse F., 1999). 


\section{Autres aspects de l'environnement}

Un mémoire est consacré aux végétaux et aux algues des eaux de la plaine de la Ruzizi. La première partie décrit le cadre physique général et l'action de l'homme, la deuxième, les différents biotopes aquatiques (Mpawenayo B., 1996).

La jacinthe d'eau (Eichornia crassipes) a été une peste végétale qui a gêné fortement la navigation sur le fleuve Congo et ses affluents à la fin de la période coloniale. Les méthodes de lutte utilisées dans les années 1950 se sont soldées par un échec. On se contenta finalement de nettoyer les parties extérieures immergées des bateaux (Lederer A., 1995). Après 1960, plus aucun budget ne fut consacré à la lutte contre la jacinthe. Cependant la situation ne s'est pas aggravée comme s'il s'était établi une sorte d'équilibre naturel (Charlier J., 1995).

La protection des milieux naturels:

le Rift occidental serait un des lieux privilégiés dans le Monde pour la diversité des oiseaux. D'où l'importance de mesures de conservation et de protection. Le taux d'endémicité le plus élevé pour les oiseaux se rencontrerait dans le plateau de l'Itombwe, au nord-ouest du lac Tanganyika (Louette, M. 2006).

La forêt de l'Itombwe serait la deuxième en importance en Afrique des forêts de montagne, avec une très forte biodiversité et un large éventail de types de forêts. II faudrait la protéger et assurer sa durabilité (Doumenge, C., 1998).

Voici une bibliographie des études (et notamment des diverses expéditions) qui se sont préoccupées des bonobos, ces petits chimpanzés de la cuvette centrale, qui ne seraient plus que 15000 en 1990 alors qu'ils étaient estimés à 54000 vingt ans plus tôt (Vink H., 2000).

II manquait une étude rappelant les conditions de création des Parcs Nationaux au Congo Belge entre les deux guerres. Elle nous est donnée par J-P. Harroy, qui a été un des secrétaires de l'Institut des Parcs nationaux et un de ses conservateurs.
Nous ne saurons pas pourtant pourquoi le directeur J.M. Derscheid, qui avait été le pionnier des Parcs pendant les années vingt, a été écarté lors de l'installation officielle de l'Institut (Harroy J.-P., 1993). La comparaison de photos prises respectivement en 1958 et en 1988 montre, dans le Parc National des Virunga (ex Parc Albert), l'évolution de la végétation suite à l'arrêt des feux de brousse (savanes envahies par des buissons d'Acacia) et les évolutions différentielles de part et d'autre de la limite du parc. Occupation totale par les cultures des espaces limitrophes du côté extérieur, reforestation de l'espace intérieur (Verschuren J., 1993). Un des points positifs des gouvernements congolais/zaïrois et aussi de ceux du Rwanda et du Burundi avait été de maintenir et même de multiplier leurs Parcs Nationaux. Ils devraient pour poursuivre dans cette direction bénéficier de l'aide internationale (Hecq J., 1994). L'arrivée en 1994 de plusieurs centaines de milliers de réfugiés rwandais en bordure du Parc et les opérations militaires, qui ont suivi, ont entraîné, avec une forte recrudescence du braconnage, la menace de disparition des animaux rares du parc des Virunga (gorilles de montagne sur les volcans de la frontière, éléphants). II y a eu un véritable massacre d'hippopotames. De graves dégâts ont été commis à la végétation forestière et les cultures vivrières ont empiété sur le territoire du Parc. Certains en parlent comme d'un des grands désastres écologiques de la fin du XXe siècle (Lelo Nzuzi, 1999). Les images satellitaires ont été utilisées pour suivre une phase particulièrement rapide de déforestation en 2004. Grâce à l'action combinée d'organismes divers, cette phase a pu être arrêtée. La réhabilitation est suivie par le recours systématique à ces images (de Wasseige C., 2006).

Voici une série de réflexions sur les stratégies à mettre en oeuvre pour combattre le braconnage et réglementer la chasse en vue d'une gestion durable des ressources fauniques (Monsembula J.C., 2005). 


\section{LA GÉOGRAPHIE DE LA SANTÉ}

Ici aussi, on pourrait dire que les thèmes n'ont guère changé et que les situations sur place ont fort peu progressé. Certaines se sont même aggravées. Nous ne prendrons en considération que des publications qui s'occupent plus précisément d'épidémiologie et de santé publique en laissant donc de côté tout ce qui concerne la recherche fondamentale et la recherche thérapeutique.

On notera la disparition depuis 1996 des Annales de la Société belge de Médecine tropicale, qui étaient étroitement liées à I'Institut de Médecine Tropicale d'Anvers, et même l'effacement progressif de la société éditrice. Certes les questions, que l'on y traitait, ont été reprises par une revue internationale, Tropical Medicine and International Health, publiée par la maison d'édition scientifique Blackwell, dont le premier numéro est sorti en février 1996 mais elle n'a pas la même spécificité centrafricaine sinon congolaise. Nous ne l'avons pas dépouillée de façon systématique.

\section{LES MALADIES DITES TROPICALES}

\section{Le paludisme}

Dans la lutte contre le paludisme, on continue à préconiser la distribution dans les villages de moustiquaires imprégnées d'insecticides. Une expérimentation faite en 1991 sur les plateaux Bateke, à 60 km à l'est de Kinshasa, a montré que la densité des populations d'Anopheles gambiae avait diminué de $94 \%$ dans le village protégé par ces moustiquaires par rapport à deux villages voisins non protégés. La prévalence du paludisme a baissé à partir du cinquième mois. Les résultats ont donc été encourageants (Karch S. et al., 1993).

Des moustiquaires imprégnées ont été distribuées à bas prix (2 US \$), vers 1995, dans la région de l'Imbo, au Burundi, c'est-à-dire dans une partie basse du pays, en bordure du lac Tanganyika (commune de Nyanza-Lac). Les résultats ont été très irréguliers. Les moustiquaires ont connu plus de succès dans les terres les plus basses et moins de succès dans les terres situés à altitude plus élevée. Mais beaucoup semblent avoir été revendues dans les pays voisins. II faudra améliorer la diffusion de l'information en associant le personnel de santé, les autorités locales et les instituteurs (Van Bortel W. et al., 1996).

Une forte prévalence de l'anémie et l'existence de bas niveaux d'hémoglobine chez les jeunes enfants examinés à Kivunda (Territoire de Luozi, Bas-Congo) seraient-elles liées à l'importance de la malaria? à une carence en fer? à une carence protéo-énergétique? (Kuvibidila S. et al., 1993).

A Katana, sur le lac Kivu, un peu au nord de Bukavu, on a relevé, entre janvier 1985 et mars 1986, de nombreux cas d'hémoglobinurie et de blackwater fever. Cela a coïncidé avec l'utilisation accrue de quinine suite au développement de la résistance du parasite malarien à la chloroquine. La blackwater fever serait une complication de la malaria à $\mathrm{P}$. falciparum en relation avec l'usage de quinine (Delacollecte C., Taelman H. et Wéry M. (1995).

\section{La maladie du sommeil}

Sur la maladie du sommeil, voici deux études à caractère historique de P.G. Janssens.

Les autorités médicales coloniales ont eu recours à des méthodes parfois vigoureuses pour la combattre. L'action d'un de ces médecins, Emile Lejeune, comparée à celle d'Eugène Jamot dans l'Afrique française, est décrite à partir d'un texte qu'il a consacré à la prophylaxie de la maladie du sommeil au Congo belge en 1923. On trouvera, dans le même article, une description de la méthode utilisée par J. Schwetz, à la même époque, en milieu hyperinfecté (par exemple dans le Kwango-Kwilu) (Janssens P.G., 1995). Le deuxième article nous donne quelques indications sur l'apparition de la trypanosomiase et ses ravages dans le BasCongo et le bassin de la Cwanza (Angola) 
en utilisant le rapport d'un médecin portugais chargé en 1900-1901 d'évaluer l'importance de la maladie qu'on estimait responsable du déclin des plantations de la Cwanza. On pensait alors que la maladie était d'origine bactérienne et que l'épidémie, qui aurait commencé trois décennies plus tôt, était en voie d'extinction. La première mention écrite de l'existence de la maladie au Congo date de 1859. Un chirurgien français la décrit parmi les «émigrants» (c'est-à-dire les esclaves) des factoreries de l'embouchure du fleuve (Janssens P.G., 1996). Voir aussi sur ce sujet l'article de P. Salmon (1995), mentionné dans la partie historique de cette chronique.

Le nombre de cas nouveaux de trypanosomiase a doublé, des années 70 à la fin des années 80. Chez les pêcheurs Bayanzi, sur les rives du Kasai (Zone de Bagata, Bandundu), un traitement à la pentamidine avait pratiquement fait disparaître l'endémie dans les années 50. Une forte recrudescence s'est produite au début des années 70 et a connu son apogée en 1978. On a appliqué une nouvelle méthode de dépistage (par tests sérologiques) et de traitement des habitants de trois villages. Les résultats ont été satisfaisants mais cinq années plus tard (en 1992), on est revenu au même niveau d'infection. Tout cela est fort décourageant (Bruneel H. et al., 1994)

La recrudescence de la trypanosomiase s'est accrue encore après 1990 suite à la fin de la coopération belge et du changement de statut du Fonds Médicat tropical (FOMETRO), devenu simple ONG. La dégradation des systèmes de santé de base a aggravé la situation. II faut chercher de nouvelles approches pour reprendre la lutte de façon moins onéreuse et avec une dépendance moindre visà-vis de l'aide extérieure (communication de Gigase P., 1998, à l'ARSOM en 1997, non publiée).

\section{L'onchocercose}

On a délimité trois foyers d'onchocercose au Burundi. Le plus vaste, au nord-ouest, menace 360000 personnes. Un deuxième, dans le sud-ouest, en menace
230 000. II existe aussi un petit foyer dans le sud-est (52 000 personnes à risque) (Newell E., 1995).

\section{LE SIDA}

Le Sida continue ses ravages. Le virus est largement disséminé à bas niveau aussi bien en milieu rural qu'en milieu urbain mais il investit de plus en plus fortement les métropoles et les villes secondaires. Certains pensent que, dans une grande partie de l'Afrique centrale, donc du Congo, on a affaire à un champ endémique ancien autant rural qu'urbain mais que la montée de la prévalence à Kinshasa, par exemple, serait peut-être due à l'apparition de nouveaux virus (ou de souches nouvelles?) (Remy G., 1993). Dans l'Afrique au sud du Sahara, l'infection VIH/Sida se caractérise par les valeurs élevées du taux de transmission hétérosexuelle, du taux de prévalence moyen, du taux de transmission du VIH de la mère à l'enfant. Au cours de la décennie 1990, l'épidémie a déferlé sur l'Afrique australe. Mais il y a peut-être en fait plusieurs épidémies distinctes. L'impact majeur de l'épidémie est peutêtre encore à venir (Amat-Roze J.-M., 1995 et 2001).

L'historien John Iliffe, qui nous avait donné une brillante étude sur la pauvreté en Afrique, a consacré un livre à l'histoire de l'épidémie africaine de Sida. II a cherché à répondre ainsi à la question que posait le président Thomas Mbeki, d'Afrique du Sud, quand celui-ci déclarait que l'épidémie était liée principalement à la pauvreté et à l'exploitation de la population africaine. A ce moment, d'autres mettaient en cause un système sexuel propre aux Africains. J. Iliffe montre brillamment que l'histoire du Sida en Afrique s'inscrit, au départ, dans le processus de la pénétration humaine de l'écosystème naturel de la partie occidentale de l'Afrique centrale et que le développement épidémique est lié à la croissance démographique, à l'expansion urbaine et aux changements sociaux (Iliffe J., 2006).

L'anthropologue Daniel Vangroenweghe a consacré un livre au même thème général 
du Sida en Afrique. Dans la première partie, il retrace I'histoire mondiale du Sida et fait notamment une longue analyse critique de l'hypothèse selon laquelle le mode de fabrication de vaccins antipoliomyélitiques au Congo à la fin des années 1950 et leur utilisation dans une grande campagne de vaccination seraient responsables de son développement dans ce pays. La deuxième partie étudie les comportements sexuels dans les différentes parties de l'Afrique tropicale avec notamment comme objectif de déterminer s'ils ne rendent pas certaines sociétés plus vulnérables que d'autres (Vangroenweghe D., 1997 et 2000).

A l'occasion de la remise du Prix Roi Baudouin à une organisation ougandaise de prévention du Sida et d'accompagnement des malades (The Aids Support Organization TASO), plusieurs communications concernent le Sida en Afrique (SIDA et développement en Afrique, 1995). Outre celle d'Amat-Roze déjà citée, on retiendra celle de $Y$. Verhasselt sur le fonctionnement de cette organisation (pp. 21-26), celle de P. Bouvier concernant les conséquences du Sida sur les structures socio-parentales ( $p p$. 39-53) et celle de G. Carrin, traitant ses effets sur les ménages ruraux (pp. 55-78). Voici quelques précisions sur les dates possibles d'apparition du Sida au Congo. Elles confirment que l'épidémie est née et s'est développé à partir de l'Afrique centrale. Dans notre chronique précédente, nous avions cité la découverte du virus dans le sérum d'une femme congolaise traitée à Bruxelles en 1977. On en déduisait que le virus avait dû apparaitre dans la première moitié du XXe siècle. Depuis lors, on a découvert des fragments de virus dans des biopsies de ganglions lymphatiques effectuées en 1960 à Léopoldville, ce qui a permis d'analyser une partie du génome d'une souche $\mathrm{VIH}-1$ (DRC 60). Des séquences virales ont été découvertes dans un plasma sanguin datant de 1959 à I'hôpital universitaire de Kinshasa (ZR 59). Les deux souches étaient différentes, leurs génomes différant de $12 \%$. On estime qu'elles ont dû avoir un ancêtre commun au plus tard 50 ans plus tôt soit donc au début du XXe siècle. Une équipe d'une douzaine de chercheurs américains, congolais, belges, néerlandais, australiens, etc., a analysé à l'Université d'Arizona, 813 échantillons histopathologiques conservés dans la paraffine dans les archives du Département d'anatomie et de pathologie de l'Université de Kinshasa et datant de 1958 à 1962. A l'issue d'un traitement mathématique tenant compte de la vitesse des différenciations, cette équipe a conclu que l'ancêtre commun a dû apparaître entre 1902 et 1921. C'est à Kinshasa qu'on observe aussi la plus grande diversité des virus du groupe $M$, qui est responsable de $95 \%$ des infections $\mathrm{VIH}$ dans le monde. Comme les souches VIS (virus d'immunodéficience simiesque) les plus proches du groupe $M$, ont été trouvées chez des chimpanzés de l'extrême sud-est du Cameroun (bassin de la Sangha, donc au coeur de la cuvette congolaise), à $700 \mathrm{~km}$ de Kinshasa, on peut supposer que c'est là qu'il y a eu transfert du virus à l'homme, par le sang de chimpanzé, chez des chasseurs préparant de la viande de chimpanzé pour la consommation. Mais pour que l'épidémie se développât, il a fallu attendre que le virus arrive, par les déplacements le long des rivières, dans des noyaux suffisamment denses de population. II les a trouvés dans les agglomérations qui commencent à croître à cette époque. L'expansion sera exponentielle: quelques individus concernés vers 1910, quelques milliers vers 1960, tous en Afrique centrale (à ce moment, il y aura transfert de souches du sous-type B, par Haïti, vers l'Amérique du Nord puis l'Europe), 55 millions dans le monde en 2007 (Sharp P. et Hahn B., 2008; Worebey M. et al., 2008).

Voici le cas d'une petite ville de 40000 habitants (Inongo, sur le lac Mayi Ndombe), donc assez proche du milieu rural. La prévalence brute est de 1,1\%, un peu plus forte chez les femmes que chez les hommes. Une campagne de prévention devrait viser d'abord les migrants masculins (travailleurs saisonniers et itinérants) (Garin B. et al., 1993). Au Rwanda, l'épidémie a progressé de 91\% par an de 1983 à 1990. Les deux tiers des malades dépistés étaient dans les villes et près des bourgs de services 
le long des axes commerciaux. La maladie est une grave menace pour le pays. Au moment où l'article, qui cite ces données, a été écrit, certains n'étaient pas loin, dans le pays, de considérer l'épidémie comme un moyen pour le Rwanda, avec la guerre, de rajuster sa densité démographique à ses ressources! C'était avant le drame de 1994 (Gotanègre J.F., 1993).

Une enquête menée auprès d'un groupe d'adolescents au Rwanda, dans cing écoles secondaires de la province de Gitarama, montre que les interventions de prévention du Sida, qui leur ont été destinées, ont eu peu d'effets sur leur comportement sexuel. La pandémie de sida a un effet dévastateur sur l'organisation de l'enseignement, en frappant les élèves et le corps enseignant (Michielsen K., Bosmans M., Temmerman M., 2008).

La lutte contre le Sida peut être perturbée par l'attitude de la population envers la maladie. Dans la Zambie voisine, le Sida est considéré par certaines Eglises chrétiennes comme un signe annonciateur du jugement dernier (Gausset Q. 2001). Au Congo, alors que la maladie du sommeil avait été considérée comme la maladie coloniale, apportée par le colonisateur belge, le Sida a été associé à l'indépendance puis considéré comme la maladie de Mobutu, c'est-à-dire apportée par son régime.

Dans un numéro de Congo-Afrique consacré uniquement au Sida en RDC et en Afrique, on lira un article extrait d'un mémoire de licence en sciences géographiques de l'Université de Lubumbashi, qui a utilisé les données de cinq cliniques et de deux centres de dépistage de cette ville de janvier 2003 à septembre 2005. Les taux de prévalence ont peu varié au cours de ces trois années mais ils sont assez différents d'un quartier à l'autre. La tranche d'âge la plus affectée est celle de 35 à 43 ans, les groupes d'activités les plus affectés sont les gérants de bars, restaurants, hôtels puis les fonctionnaires, particulièrement ceux des services des douanes et des migrations (Insoni A., 2007).

\section{LES MALADIES VIRALES NOUVELLES}

Parmi celles qui ont ravagé I'Afrique centrale, la fièvre Ebola a sans doute semé le plus d'effroi. Nous ne disposons pas cependant d'études permettant de lier cette maladie aux conditions du milieu congolais, sinon l'existence probable d'un réservoir du virus parmi les chimpanzés. Dans une autre partie de notre chronique, on pourra voir comment la maladie a pu bouleverser les rites funéraires et l'attitude envers les morts. II semble d'autre part, que le transport clandestin de mourants ou de cadavres vers leur village d'origine, pour accomplir les rites funéraires traditionnels, notamment lors de l'épidémie de Kikwit en 1995, a dû favoriser la diffusion de la maladie.

\section{PERSISTANCE ET RECRUDESCENCE DE LA TUBERCULOSE}

Bien que la situation troublée, qui a persisté dans plusieurs régions du Congo, n'ait pas permis de mesurer l'intensité actuelle du phénomène, il est évident que la tuberculose reste une menace grave d'autant plus qu'elle est souvent associée au Sida. Or la prévalence de celui-ci est estimée à 4,2\% de la population de 15 à 49 ans et a dû augmenter dans les territoires parcourus par les troupes armées. La résistance aux antibiotiques antituberculeux s'est accrue. On a estimé l'incidence annuelle de la tuberculose à 160 cas pour 100000 habitants (Kayembe Kalambayi P. \& Kimpanga Dianga P., 2006).

\section{LA MALNUTRITION ET LES MALADIES DE CARENCE}

La malnutrition infantile et les maladies de carence restent un gros problème dans les provinces du Kivu. Les interventions nutritionnelles au niveau du couple mère-enfant contribuent largement à améliorer l'état de l'enfant mais il faut en même temps améliorer la situation sanitaire générale par une politique de soins de santé primaires impliquant une participation de la communauté concernée (Hennart Ph., Porignon D., Donnen Ph., 1996) 
Comme on a constaté, dans la zone de santé rurale de Kapolowe, au Katanga (projet CIF «Village Katanga»), que les enfants, qui avaient été traités à l'hôpital pour malnutrition, étaient victimes de rechutes fréquentes, on a proposé un programme de réhabilitation nutritionnelle à domicile en y faisant participer les familles. On les aide à identifier les causes profondes de la malnutrition et on favorise des réseaux collectifs d'entraide pour acquérir les aliments recommandés pour enrichir la bouillie quotidienne (Tellier V. et al., 1996). La dégradation de la situation nutritionnelle dans la Région des Grands Lacs doit être considérée comme un des facteurs de la crise politique de 1994 (Vis H., 1997 b). Dans cette région, de nombreux problèmes sanitaires découlent de la malnutrition ou contribuent à la renforcer (charges infectieuses bactériennes, parasitaires et virales, mauvaises conditions d'hygiène voir les reprises des endémies de gastroentérite à vibrion cholérique et à shigella, etc.). En outre, les troubles ont dégradé les systèmes de prévention et de médecine curative (Goyens P., 1997).

La malnutrition sévit aussi dans les villes. Des enquêtes anthropométriques faites de novembre 1991 à mars 1994, à Kinshasa, ont montré une fluctuation saisonnière de la malnutrition en relation avec les prix des produits vivriers sur les marchés et une tendance générale à la hausse. En mars 1994, 10,7\% des enfants examinés se trouvaient dans une situation de malnutrition globale aiguë. Ce sont évidemment les enfants des milieux les plus défavorisés (Arbyn M. et al., 1995).

L'aide à la nutrition ne devrait-elle pas tenir compte du problème de l'intolérance au lactose que l'on rencontre dans une partie de la population de la région des Grands Lacs? La guerre et les déplacements de populations y ont engendré une situation endémique de malnutrition. Les enfants lactose-intolérants (cette intolérance aurait une origine génétique) souffrent davantage de cette situation que les lactose-tolérants qui récupèrent mieux quand l'état nutritionnel s'améliore. II faudrait tenir compte de ces faits quand on envisage une aide alimentaire par apport de lait (Brasseur D., Goyens Ph. et Vis H., 1998).
La carence en iode a été combattue par la distribution de sel iodé à la population. Une évaluation faite au cours de la dernière décennie en RDC et en RCA a montré une régression très significative du goitre, la disparition du crétinisme mais aussi le développement de cas d'hyperthyroïdie et de maladies auto-immunes thyroïdiennes inconnues auparavant dans la région. L'OMS devrait s'inspirer de l'exemple de la Suisse qui a su résoudre ses problèmes de carence en iode avec un minimum d'effets secondaires (Bourdoux P. et al., 2005).

\section{LE FONCTIONNEMENT DES SYSTÈMES DE SANTÉ}

Les deux dernières décennies du vingtième siècle ont vu l'application, dans une bonne partie des pays en développement, des résolutions d'Alma Alta (1978) sur la Santé pour Tous à l'Horizon 2000, c'est-àdire d'une politique généralisée de soins de santé primaires. Parmi les essais, qui ont été menés dans différents pays pour aboutir à la formulation de ce modèle, figure le projet de recherche-action de Kasongo, au Maniema, entrepris par l'Institut de Médecine Tropicale d'Anvers. Celui-ci avait repris les activités hospitalières de Kasongo peu avant l'indépendance du Congo (une partie, dont le sanatorium, était gérée auparavant par le Cemubac) et y avait développé un système de santé intégré au service du développement général: un réseau de centres de santé, un hôpital de référence, une pharmacie, une contribution financière forfaitaire des prestataires. Dans le périmètre couvert, $90 \%$ de la population étaient inscrits dans les centres de santé à la fin des années 80 mais la dégradation générale de l'économie rendait difficile le fonctionnement de beaucoup de ceux-ci. L'arrêt de la coopération belge après 1990 a compromis tout le système (Van Balen $\mathrm{H}$., 1997).

Dans certaines régions, ce système de centres de santé, qui comportait une participation active de la population locale, a continué à fonctionner souvent avec l'aide indirecte et les conseils de diverses ONG. Mais dans l'est du Congo, il a été confronté à une situation de guerre. Un 
événement essentiel a été d'abord, à la suite des mouvements de réfugiés et des épidémies diverses survenant dans leurs camps, l'intervention de grandes organisations internationales dotées de moyens puissants, installant des hôpitaux temporaires, distribuant gratuitement nourriture et médicaments (Croix-Rouge Internationale, Croix-Rouge allemande, Médecins Sans Frontières, organisations onusiennes de secours aux réfugiés, etc.). Beaucoup de réfugiés se sont aussi installés en dehors des camps. Comment les systèmes de santé, qui étaient en place, se sont-ils comportés face à ces interventions? Certains de leurs propres équipements, qui fonctionnaient encore correctement, ont souvent été utilisés par les organismes internationaux. Mais la démarche de ceux-ci est par définition temporaire, essentiellement dans l'urgence. Dans quelle mesure peut-elle se concilier avec une action de développement sanitaire qui se fait dans la durée? De façon générale aussi l'aide humanitaire ne répond-elle pas à une autre problématique que l'aide au développement? Ces questions sont soulevées dans plusieurs articles qui montrent une bonne résistance de certaines zones de santé comme celles du district de Rutshuru (qui a traité 65000 cas de pathologie parmi les réfugiés rwandais installés en dehors des camps) ou de Kirotshe, toutes deux dans le Nord-Kivu. Le district de santé s'est révélé une structure efficace pour faire face à des conditions exceptionnelles. Citons, à titre d'exemple, les articles de Porignon D. et al., 1995, Vis H. et Goyens P., 1996, Goyens P. et al., 1996, Porignon D. et al., 1998. Un article de Porignon D. et al. (2006) revient sur le sujet de la «résilience», c'est-à-dire la capacité à résister et à «rebondir», des zones de santé en traitant particulièrement le cas du district de Rutshuru.

Les organismes, qui sont intervenus dans l'aide humanitaire d'urgence, connaissaient mal les problèmes sanitaires et nutritionnels locaux, ce qui les a conduits à commettre des erreurs de diagnostic et de prise en charge. Cela a été particulièrement vrai pour l'épidémie de choléra qui a sévi parmi les réfugiés à Goma. Or des épidémies de choléra avaient été surmontées efficacement dans la même région quelques années auparavant, notamment en 1978, grâce à des études biologiques approfondies qui avaient permis l'utilisation de solutions de réhydratation adaptées à la situation de malnutrition chronique dans laquelle se trouvaient les malades. Une prise en considération insuffisante de cette situation à Goma en 1994 et le fait que les équipes d'urgence n'ont pas tenu compte des analyses et des études antérieures - ou ne les connaissaient pas - ont certainement agi sur le nombre des victimes (Vis $H$. et Goyens P., 1996, Goma Epidemiology Group, 1995, Siddique A. K. et al., 1995). Comment la recherche en santé peut-elle être menée aujourd'hui dans les conditions particulièrement difficiles de l'est du Congo? Une équipe du Cemubac expose la méthodologie qu'elle a mise au point (Porignon D. et al., 2006).

II est intéressant de suivre sur près de deux décennies l'évolution des décès maternels, des décès de nouveaux-nés et des naissances de faible poids dans une maternité du Nord-Kivu (Rutshuru) (Mugisho E. et al., 2002).

Dans la zone de santé de Ngaba, à Kinshasa, on a voulu vérifier si l'accessibilité aux soins de santé ne dépendait pas aussi d'autres facteurs que le revenu. A un certain niveau de revenu, d'autres facteurs interviennent pour expliquer les différences dans le recours à ces soins. II s'agit notamment de la religion et de la prise en charge des soins de santé par une association. Les membres de religions «classiques» (catholiques, protestants, kimbanguistes, musulmans) ont une accessibilité cinq fois supérieure à celle des membres des religions africaines (Eglise des Noirs en Afrique, Bundu dia Kongo, etc.). Parmi les membres des Eglises de réveil (Témoins de Jéhovah, etc.), certains s'en remettent à la prière pour obtenir la guérison (Kebela Kebela P., 2004). Pour celles-ci, voir notamment les articles de R. Devisch cités plus loin 


\section{LA DÉMOGRAPHIE ET LA GÉOGRAPHIE DE LA POPULATION}

\section{LES DONNÉES ANCIENNES}

On sait que la valeur des données démographiques en Afrique a été le plus souvent fort médiocre. J. Stengers nous rappelle les différents facteurs qui ont pu affecter leur qualité. II n'est pas toujours certain non plus que les données les plus récentes soient nécessairement plus dignes de confiance que les données anciennes (Stengers J., 1992). Nous pourrions ajouter que, pour les opérations de dénombrement et d'enrôlement des électeurs du référendum sur la nouvelle constitution et des premières élections de la RDC, qui équivalaient à un recensement d'une partie de la population (celle de plus de 18 ans), certaines relèvent du surréalisme, comme le transport, en pirogue parfois, vers des régions isolées (et bien sûr dépourvues de toute ressource électrique), de matériel informatique pour la confection de cartes d'électeur électroniques. Mais la communauté internationale n'avait pas voulu lésiner sur les moyens financiers pour réussir la «démocratisation» du pays!

On se réjouira de la réédition du livre qu'Anatole Romaniuk avait publié en 1961 et qui synthétisait la grande enquête sociodémographique qu'il avait dirigée de 1955 à 1957. Elle donnait enfin une vue précise de la situation dans les dernières années de la période coloniale. Cette enquête par échantillonnage (10 \% de la population rurale, $15 \%$ de la population urbaine), la plus vaste à avoir été organisée au Congo belge, a été menée voici donc un demi-siècle, à un moment où la perspective d'une diminution de la population préoccupait encore certains esprits. Elle avait révélé que le pays avait, dans son ensemble, un taux de croissance naturelle de 2,3\% (1,8\% pour le milieu rural, $4,3 \%$ pour le milieu urbain et $3,7 \%$ pour le milieu mixte). Elle avait confirmé l'existence de poches de faible croissance et de médiocre natalité dans certains districts de la Province Orientale (Bas et Haut-Uele) et de la Province de l'Equateur (Tshuapa). Le travail avait été effectué avec maîtrise et enthousiasme. L'enquête n'avait pas été confiée à des agents coloniaux territoriaux, comme c'était le cas auparavant, mais à des équipes de fonctionnaires africains, spécialement formés pour cette opération. Les données de cette enquête constituent les références essentielles pour toutes les études démographiques ultérieures même si certains ont suggéré que ses résultats comportaient une part de sous-estimation parce que, en situation coloniale, une partie de la population aurait cherché à se soustraire aux enquêtes (Romaniuk A., 2006).

La politique sociale de l'Union Minière du Haut-Katanga, qui avait créé une infrastructure médico-sociale, construit des logements et qui intervenait dans l'alimentation de ses travailleurs en fournissant une ration alimentaire (remplacée partiellement par un versement en argent après 1950, supprimée en 1961 puis réinstaurée sous la forme d'un accès à des cantines, c'est-à-dire des magasins à prix réduits en 1964) avait fait diminuer fortement les taux de mortalité infantile dans les familles qui dépendaient d'elle. De 316 pour mille en 1929-31, la mortalité infantile était tombée à 160 en 1938-40 et 95 pour mille en 1947-49. Son minimum a été de 51 pour mille en 1959-61. Elle remonte ensuite à 66 pour mille en 196567 pour redescendre aux environs de 61 pour mille en 1968-1970 (Dibwe dia Mwembu, 1993).

\section{LES DONNÉES DÉMOGRAPHIQUES ACTUELLES}

Le vieillissement de la population, sinon même tout simplement la transition démographique, n'est pas encore sensible au Congo. Le pourcentage des personnes de plus de 64 ans est de 2,8 seulement en 2000 et ne dépassera pas 5,6 en 2025. L'espérance de vie reste faible: 47 ans pour les hommes et 49 ans pour les femmes en 1998. Elle n'atteindrait 70 ans qu'en 2050 (sans tenir compte des effets du Sida!). Le taux de fécondité ne faiblit 
pas. Le taux de croissance naturelle est passé de 1,2\% en 1950 à 3,1\% en 1998 . La femme congolaise passe sa brève vie dans les maternités, l'éducation et la scolarisation de ses six enfants et meurt le plus souvent avant la fin de la scolarisation de son dernier enfant (Ngondo a Pitshandenge S., 2001).

La mortalité maternelle atteindrait des taux effrayants. Une enquête de 1998 aboutit à un taux de 1837 décès maternels pour 100000 naissances vivantes alors que la valeur calculée par le PNUD pour la décennie 1980 était de 870 (une enquête du CEMUBAC avait fourni la valeur de 750 pour les zones rurales du Kivu, en 1982-83) et que, pour les autres pays de l'Afrique tropicale, il varierait entre 215 et 960 . Faut-il croire à un effet combiné de la crise économique et du Sida (détérioration des conditions économiques et alimentaires, augmentation des avortements à risques par suite du manque de moyens contraceptifs, manque d'accès financier aux soins) ou bien ces chiffres catastrophiques sont-ils la conséquence de la méthode suivie pour le calcul ou d'une trop petite taille de l'échantillon? Les taux de mortalité infantile auraient par contre diminué entre1984 et 1998 sauf dans l'Equateur, les deux Kivu, le Bas-Congo et le Maniema. Le taux de natalité à Kinshasa serait passé, pour la même période, de 5,14 à 2,8 \%! (Ngondo a Pitshandenge S., 2000). On devine que tous ces chiffres peuvent être sujets à contestation.

Les démographes, après les décisions de la Conférence Nationale Souveraine, ont été très inquiets sur la façon dont pouvait être réalisé le recensement préalable à des élections générales. Comment traiter en effet le problème de l'identification, c'est-à-dire concrètement de la distinction entre les habitants de nationalité congolaise et les étrangers? Dans le Kivu notamment, si on laisse de côté le problème des réfugiés de fraîche date qui sont parqués dans des camps, un simple fonctionnaire pourra-t-il refuser la reconnaissance de la nationalité congolaise à un immigrant de vieille souche, cette décision risquant d'avoir un effet de vie ou de mort pour la personne concernée et sa famille? Les procédures prévues par la Conférence Nationale Souveraine sont apparues très vite comme inapplicables (Ngondo a Pitshandenge S., 1995). Nous n'avons pas d'information précise sur ce qui a été fait. Nous avions nous-même évoqué ce problème à propos du recensement de 1984, qui contrairement à toute évidence, indiquait très peu d'étrangers dans le Kivu (voir Nicolaï H., 1998, pp. 15-18)

Nous retrouvons, une dizaine d'années plus tard, exprimées cette fois par L. de Saint Moulin et plus précisément du point de vue démographique, les inquiétudes que l'on pouvait nourrir sur les résultats qui sortiraient des opérations d'inscription des électeurs pour les élections prévues en 2006. D'autant plus qu'un essai de recensement de la population dans une commune de Kinshasa avait donné des résultats peu fiables et qu'une enquête sur la perception des élections par les habitants, indiquait qu'une très forte proportion des personnes enquêtées (46\%) craignaient que les perdants des futures élections ne recourent à des tentatives de rébellion (de Saint Moulin L., 2005). Le même auteur rappelle, dans un autre article, cité à propos de la préparation des élections (de Saint Moulin L., 2006 a), que la population totale du Congo, attendue pour 2005, était de 59 millions d'habitants, étrangers non compris.

Sur la géographie et l'histoire de la population proprement dite, L. de Saint Moulin a continué à nous fournir régulièrement des mises au point et des précisions nouvelles. Voici d'abord un article sur l'évolution des densités. Y a-t-il eu concentration accrue de la population entre 1948 et 1984? Oui si l'on envisage l'accroissement de la part de la population urbaine. Mais, dans le milieu rural, la courbe de concentration est restée fondamentalement la même. II n'y a eu que de faibles mouvements de concentration. Par province (= région), la croissance la plus forte a été enregistrée pour les deux périodes 1948-1970 et 1970-1984, par le Kivu-Maniema; la plus faible, par le HautZaïre (Province Orientale), connu pour ses poches de faible natalité. La croissance du Kivu est liée en grande partie à 
une forte immigration rwandaise. Le Shaba (Katanga) a connu une très forte croissance pendant la première période, une croissance beaucoup plus faible ensuite. Le Kasai oriental a eu la croissance la plus forte, après celle du Kivu, pendant la période 1970-1984 (retour de Kasaiens du Shaba et développement de la production minière artisanale de diamant). II y a eu des glissements de population vers les zones d'agriculture vivrière commercialisable et les zones d'exploitation artisanale de matières précieuses. La croissance de la population rurale dans l'ensemble a été manifestement réduite par l'exode (de Saint Moulin L., 1995).

Poursuivant la série des cartes au 1/1000 000 de la répartition et de la densité de la population couvrant les différentes provinces du Congo, dont quatre avaient été dressées à la fin des années 50 et au début des années 60 dans le cadre du CEMUBAC, nous avons établi celles du Kivu (Nord et Sud Kivu) à partir des données du recensement de 1984. Une carte donne la population par localité. L'autre fournit la densité calculée par mailles géométriques. II n'a pas été possible de calculer les densités par groupements ou collectivités comme cela avait été fait pour les cartes précédentes car nous n'avons pu en connaître les limites. Un mémoire, qui comporte d'abord une analyse ces cartes, critique les données du recensement et décrit les variations spatiales de la densité. II analyse les facteurs explicatifs des fortes densités de la partie orientale (versant intérieur du Rift centrafricain avec des extensions vers l'ouest, dans le Nord-Kivu (Territoire de Masisi) et, dans le Sud-Kivu, à hauteur de Bukavu, et insiste sur l'importance de l'immigration à partir du Rwanda. Ces provinces connaissent le taux d'urbanisation le plus bas du Congo. Cependant un chapelet de localités s'égrène du nord au sud dont certaines comptent en 1984 déjà plusieurs centaines de milliers d'habitants (Uvira, Bukavu, Goma, Butembo, Beni). Certaines surfaces connaissent une charge (surcharge?) démographique comparable à celles du Rwanda voisin. Un chapitre décrit les modifications apportées à la situation depuis le recensement de 1984 (conséquences des événements du Rwanda, flux et reflux de plus d'un million de réfugiés) (Nicolaï $\mathrm{H} .$, 1998).

Les problèmes démographiques du Rwanda ont fait l'objet de plusieurs études approfondies. La plus importante est la thèse que John May a soutenue à la Sorbonne en 1996, et dont les grandes lignes sont reprises dans un article de la revue Population. La thèse s'intitulait «Urgences et négligences: pressions démographiques et réponses politiques au Rwanda (1962-1994)». L'auteur montre, après une étude très détaillée et très précise de l'évolution démographique du Rwanda indépendant, que, dans le courant des années 1980, toutes les soupapes qui avaient permis de soulager la pression démographique (récupération des terres occupées jadis par les éleveurs Tutsi réfugiés en Uganda, intensification de l'agriculture, mise en place de fronts pionniers dans les régions orientales encore peu peuplées) étaient à la limite de leurs possibilités de fonctionnement. Or, elles avaient conforté les autorités rwandaises dans leurs réticences à adopter une politique efficace de contrôle de la natalité, en grande partie d'ailleurs à cause de l'opposition de l'Eglise catholique. Une timide tentative de planification familiale avait commencé seulement en 1987. Elle n'avait pu encore porter tous ses fruits bien qu'elle eût permis de faire baisser l'indice conjoncturel de fécondité de 8,5 enfants par femme en 1983 à 6,2 en 1992 (May J., 1995 et 1996). Sur le cas du Burundi, voir le livre de Ch. Thibon (2004).

La perception par les populations paysannes rwandaises du problème démographique a été un facteur du drame de 1994. La peur de devoir partager des terres considérées déjà comme trop exiguës a été utilisée par les hommes politiques pour déclencher les actions des masses paysannes hutu contre les Tutsi. Nous avons commenté ces questions à l'occasion d'un colloque organisé par I'ARSOM. Nous y décrivons les implications démographiques de la catastrophe de 1994 d'abord pour le Rwanda même, ensuite pour les régions voisines du Kivu jusqu'au mouvement de retour des réfu- 
giés à la fin 1996 (Nicolaï H., 1997). Une étude faite dans le nord-ouest du Rwanda de 1988 à 1993 montre la montée de tensions très fortes dans les relations sociales résultant de la crainte d'une pénurie de terres (André C. et Platteau
J.P., 1997). On trouvera un peu plus loin dans cette chronique des considérations de même type sur les relations entre les faits démographiques et les événements historiques récents.

\section{L'HISTOIRE}

\section{GÉNÉRALITÉS}

\section{Bibliographie et sources}

Voici d'abord un ouvrage de référence indispensable: une bibliographie, qui rassemble, de façon quasi exhaustive, ce qui a été publié entre 1960 et 1996 sur l'histoire du Congo de 1880 à 1960. Elle compte près de 4000 références classées en 11 rubriques principales avec un index des auteurs (Loriaux F., Morimont F., Vellut J.-L., 1996).

L'Académie royale des Sciences d'OutreMer a commencé la publication de sa collection Fontes Historiae Africanae, qui s'insère dans un projet de publication internationale créé sous les auspices de I'Union Académique Internationale et dont l'objectif est l'édition critique de sources relatives à l'histoire de l'Afrique au sud du Sahara. Un volume est consacré aux sources des missions protestantes relatives à Simon Kimbangu (Vellut J.-L., 2005), un autre à une correspondance privée de Pierre Ryckmans (Vanderlinden J., 2007) (voir plus loin).

Un inventaire, utile pour tous ceux qui s'intéressent aux acteurs de la colonisation belge, a été établi pour les archives historiques privées du Musée royal de l'Afrique centrale (Van Schuylenbergh P., 1997).

Dans la collection des African Historical Dictionaries, le $n^{\circ} 76$ est une édition révisée du volume sur le Congo (Bobb S., 1999). D'autres fascicules revisés concernent le Burundi (1997, par Eggers E. K.) et le Rwanda.

Les histoires générales et l'historiographie

Une vaste synthèse de l'histoire du ZaïreCongo a été établie par I. Ndaywel è
Nziem. Ce travail monumental, écrit avec un souffle évident, n'évite pas tous les pièges des grandes synthèses mais est, à coup sûr, une pièce essentielle dans la bibliographie historique africaine. Peut-être lui reprochera-t-on une vision un peu idyllique de l'avenir du pays. L'auteur estime que le Congo-Zaïre a vocation de construire une fédération d'Afrique Centrale Latine avec le Congo (Brazzaville), le Gabon, la République Centrafricaine (Oubangui), le Rwanda, le Burundi et (ou des parties de) l'Angola (pp. 789-780). Cette conclusion, qui atteste d'une grande confiance dans l'avenir, est assez peu conforme à la tournure actuelle des événements (Ndaywel è Nziem I., 1997) et l'auteur la rejettera d'ailleurs ou la nuancera dans des articles postérieurs. La publication de ce livre a été l'occasion pour J.-L. Vellut d'un commentaire approfondi dans lequel il le situe dans la tradition des histoires nationales transposée à un cas africain et analyse dans quelle mesure ce livre se conforme aux idées qui ont sous-tendu ce genre historique dont il rappelle les ambiguités, les objectifs proclamés ou cachés et les dérives aux conséquences parfois redoutables (Vellut J.-L., 1999).

Un autre ouvrage général, à caractère plus politique, mérite aussi d'être signalé (Nzongola-Ntalaja G., 2003). On retiendra aussi l'aperçu général de J.-P. Peemans (1998), qui comporte une vue de l'évolution de l'économie et de la société. II existe d'autres publications du même type mais de valeur fort inégale. Nous ne pouvons les citer toutes ici.

Pour les régions orientales du Congo et les pays voisins, on lira, de J.-P. Chrétien, un ouvrage consacré à l'histoire générale de l'Afrique des Grands Lacs (Chrétien J.P., 2003). Sur la période du mandat au Burundi, avant la deuxième guerre mon- 
diale, voir Gahama J. (2001).

Voici un autre ouvrage général sur l'histoire du Congo qui intéressera par l'originalité de sa démarche. Comme l'indique le sous-titre «Empreintes d'une colonie», il étudie non pas l'histoire proprement dite du Congo, dont il trace cependant une rapide synthèse, mais son effet sur l'histoire de la Belgique, c'est-à-dire sur sa politique intérieure, sa position extérieure, son économie. II est évident, par exemple, que, sans le Congo, la Belgique n'aurait pas eu la même place, à certains moments, sur la scène internationale. Le livre décrit aussi les relations entre la Belgique et le Congo indépendant (Vanthemsche G., 2007).

Un gros ouvrage d'histoire économique et financière étudie tout spécialement les entreprises coloniales qui ont travaillé au Congo de 1885 à 1960, y compris l'action des hommes qui les ont dirigées (Buelens F., 2007).

L'auteur de l'histoire générale du ZaïreCongo citée plus haut a publié un bref article sur l'historiographie du Congo postcolonial. II y constate que les chercheurs congolais sont peu représentés, sans doute en grande partie en raison des difficultés matérielles des universités zaïroises. Mais il s'inquiète aussi du fait que ses concitoyens liraient peu (séquelle, suggère-t-il, d'une culture traditionnellement orale?) (Ndaywel è Nziem I., 1995).

Pour la situation de l'historiographie africaine en Belgique depuis 1960, un débat portant notamment sur les intentions attribuées aux historiens ou écoles d'historiens, s'est amorcé entre J.Vansina et J.-L. Vellut (2002) à la suite d'une interview du premier dans une newsletter des Africanistes belges qui lui était consacrée (Arnaut K. et Vanhee H., 2001).

Dans un numéro de la Revue belge d'histoire contemporaine consacré à L'histoire africaine. Tradition belge/ Afrikaanse geschiedenis. Belgische Traditie» édité par Castryck G., François L. et Vanhee H., deux des éditeurs expriment l'avis que les préoccupations des historiens africanistes belges se concentreraient encore trop fortement sur «les Belges au Congo", ce qui serait une source de polé- miques. II faudrait prendre davantage en considération les initiatives des Africains dans leur histoire et la continuité de leurs systèmes politiques et économiques (Vanhee H. et Castryck G., 2002).

En hommage à Frans Bontinck, ses amis et ses anciens élèves lui ont offert un volume de mélanges sous le titre « $L a$ Nouvelle Histoire du Congo". II contient nombre de textes intéressants, sur la problématique des langues nationales (par Obotela Rashidi), sur l'intérêt de l'étude des anthroponymes (par Mabiala Mantuba-Ngoma), sur l'autoreprésentation de la société «moderne» de Kinshasa, à travers les tableaux de ses peintres (par B. Jewsiewicki), sur la nationalité congolaise à l'épreuve du séisme des Grands Lacs (par Ndaywel è Nziem), et sous le régime Mobutu (par Sabakinu Kivilu), etc. Cependant, contrairement à ce que le titre général de l'ouvrage suggère, ces travaux ne se distinguent pas fondamentalement, ni par la nature des sujets traités, ni par les méthodes utilisées, des autres publications historiques de ces dernières années (Mabiala Mantuba-Ngoma P., 2004). Certains de ces articles font l'objet d'un commentaire un peu plus loin dans cette chronique

\section{L'HISTOIRE PRÉCOLONIALE}

\section{Quelques données archéologiques}

Des phytolithes ont été recueillis et analysés dans trois sites archéologiques de la forêt de l'Ituri. Les plus anciens, datés de 19000 à 10000 B.P. indiquent que la région nord-est du bassin congolais était couverte de forêts à la fin du Pléistocène quand elle a été occupée par l'homme. Depuis lors, l'occupation humaine semble avoir été continue (Mercader J. et al., 2000). D'autres articles dirigés par le même auteur utilisent ces fouilles pour reconstituer l'histoire ancienne du peuplement de la grande forêt équatoriale (Mercader J. et al., 1998) et aussi celle des deux derniers millénaires (Mercader J. et al., 2000 et 2001a). Des restes humains vieux de 800 ans ont été découverts dans un abri sous roche (Mercader J. et al., 2001b). 
E. Cornelissen (2002) étudie les effets vraisemblables qu'ont eus sur les sociétés humaines les changements de l'environnement entre 40000 et 12000 dans l'ensemble de l'Afrique centrale tandis que D.L. Schoenbrun (1994-1995) tente de préciser les changements dans l'agriculture qui se sont produits dans la région des Grands Lacs pendant la deuxième moitié du premier millénaire de l'ère chrétienne.

\section{L'expansion bantoue}

Une controverse, qui n'est pas tout à fait neuve, est plutôt une invitation à la prudence qu'une véritable avancée sur la question. II ne faudrait par parler de «l'expansion bantoue» car on ne peut établir de corrélation entre l'expansion des langues bantoues et la diffusion d'un certain nombre de techniques concernant les aliments de base et la métallurgie attestée par les données archéologiques (Vansina J., 1995).

J. Vansina, dans le chapitre de conclusion d'un ouvrage consacré à une exposition de masques d'Afrique centrale, trace cependant les grandes lignes de l'expansion bantoue au cours des deux derniers millénaires dans le bassin du Congo (bien que les masques soient absents des traces archéologiques). Les masques sont un héritage des traditions des Bantous occidentaux et la plupart étaient associés à des rites d'initiation ou de chasse. La diffusion de certains d'entre eux peut témoigner de certaines expansions politiques (relativement récentes) comme celle des Lunda dans le Kwango et le sud-est du Congo (Vansina J., 1993). J. Vansina a publié aussi plus récemment (en 2001) un ouvrage qui poursuit la reconstruction de l'histoire de la partie occidentale de l'Afrique centrale avant l'arrivée des Européens qu'il avait commencée avec son livre sur les sentiers de la forêt. Fondée sur des données archéologiques et linguistiques, cette reconstruction, très hypothétique, ambitionne d'expliquer comment les différentes sociétés sont nées et se sont diversifiées. Un part importante de l'ouvrage concerne les sociétés du nord de l'Angola et du sud du Congo (Vansina J., 2001).

L'histoire de la banane plantain peut-elle aider à reconstituer la diffusion des peuples «bantous»? L'adoption de la banane plantain aurait permis aux Bantous de coloniser la forêt équatoriale qui ne se prête ni à la céréaliculture ni à la culture de l'igname par suite de l'absence de saisons marquées. Quand la banane plantain est-elle arrivée dans les régions de la grande forêt? Comme les bananiers comestibles ne donnent pas de semences ni un pollen durable et ne comportent pas de parties ligneuses, on ne trouve pas de traces archéologiques de leur culture. L'opinion générale est que le bananier est originaire de l'Inde et qu'il s'est propagé en Afrique à partir de la côte de l'Océan Indien. En se fondant sur le degré de diversification des variétés actuelles et la fréquence des variations somatiques, mesurée à partir de quelques expériences, les auteurs de cette étude estiment qu'en suivant peut-être un itinéraire le long duquel la banane ensete était déjà connue, à une époque sans doute un peu plus humide que l'actuelle, le bananier plantain serait arrivé dans la grande forêt entre 1600 et 2000 B.P. Des considérations linguistiques suggèrent une extension ultérieure du plantain vers le sud (De Langhe E. et al., 1994-1995).

La linguistique peut-elle être utilisée pour reconstituer l'histoire de l'agriculture chez les peuples bantous? Un essai a été fait pour la domestication des cucurbitacées, en raison de l'absence jusqu'à présent de découvertes de restes floraux de calebasses, qui se conserveraient mal dans les sites archéologiques. Des populations proto-bantoues auraient eu un terme (cópa) désignant une calebasse utilisée pour la conservation de liquides, il y a 4 à 5000 ans (Bulkens A., 1998). Mais les bases de cette affirmation sont-elles vraiment solides?

Sur les relations anciennes de l'intérieur avec les régions littorales, L. de Saint Moulin signale, mais sans citer ses sources, que des «creuseurs» de diamant, sur les bords de la rivière Kwango, ont trouvé à une profondeur assez grande, des cauris attestant donc de relations avec l'Océan Indien (de Saint Moulin L., 1998). 


\section{L'utilisation des sources orales}

- Comment utiliser les traditions orales pour écrire l'histoire des peuples sans écriture?

La question préoccupe depuis longtemps les historiens de l'Afrique Noire. P.Salmon, qui l'avait discutée, en 1986, dans un ouvrage sur l'ensemble des sources de l'histoire africaine, la reprend dans une réédition posthume, enrichie de nombreux exemples nouveaux, dont plusieurs choisis en Afrique centrale (Salmon P., 2007). Sur les différents problèmes qui se posent à I'historien en Afrique, J.-P. Chrétien apporte son témoignage appuyé sur ses recherches au Burundi (sur le thème général du Burundi entre les voix du passé, les drames du présent et les clichés médiatiques), dans un ouvrage qui rassemble des articles publiés dans différentes revues (Chrétien J.-P., 1993).

Plusieurs publications de J. Vansina fournissent des exemples de la façon prudente avec laquelle il faut utiliser les sources orales, matière dans laquelle il fut pionnier. Signalons en passant la réédition récente de la traduction anglaise de son livre sur la tradition orale (Vansina J., 2006). Dans un article, il se demande pourquoi, parmi les quatre traditions qu'il avait récoltées sur deux épisodes de la résistance des Kuba à l'installation du pouvoir colonial en 1899 et en 1900, l'une, qui télescope notamment les deux épisodes, est devenue dominante. II la compare avec les rapports des chefs de poste belges impliqués dans les opérations militaires et montre que, parmi les différentes versions orales de ces événements, elle est celle qui s'inscrit le mieux dans la tradition du royaume kuba (Vansina J., 1993 a). Un article rappelle les témoignages sur I'histoire des Kuba qu'apportent les statues de leurs rois (Belepe Bope Mabintch, 2004).

Autre exemple: pour l'histoire de la conquête lunda du Kwango, J.Vansina arrive à la conclusion, à la suite de la comparaison avec des sources écrites, que les traditions relatives à Kinguri selon lesquelles celui-ci serait arrivé au Kwango au milieu du XVIe siècle, ont été inventées vers 1840. Kinguri serait venu du plateau de Benguela et n'aurait eu aucun lien avec les Lunda. L'expansion lunda se serait faite un siècle plus tard et aurait pris une centaine d'années (Vansina J., 1998 et 2000).

W. MacGaffey montre que I'histoire réelle des Kongo de Mbanza Manteka est l'inverse de celle que raconte leur tradition. Ils affirment avoir participé à un grand mouvement centrifuge issu de Mbanza Kongo. En fait, ils sont constitués d'un ensemble de petits groupes venus de différentes directions. Le modèle centrifuge est une projection dans le passé d'un idéal de centralisation politique. La tradition est donc un bricolage actuel et le présent risque ainsi de créer le passé (Macgaffey W., 1993). Dans un ouvrage plus récent, l'auteur donne son point de vue sur une remise en question de l'historiographie de l'Afrique centrale précoloniale (MacGaffey W., 2005).

Sur le poids des sources orales dans l'écriture de l'histoire du Katanga, on lira Dibwe Dia Mwembu (2004).

Dans un chapitre de supplément à la fin d'une réédition à l'identique d'un texte de 1960 sur l'histoire du royaume rwanda, J.Vansina fournit l'historiographie de la question depuis la première édition et montre la difficulté à établir une chronologie précise des règnes à partir d'une estimation de leur durée moyenne. II n'est guère possible de remonter au delà du XVIIIe siècle. II montre aussi que, depuis la première édition de son ouvrage, les interprétations sur les relations entre Tutsi, Hutu et Twa sont devenues plus complexes. La subdivision en trois groupes devient de plus en plus confuse. Notons cette opinion: "Le débat est fallacieux puisque les groupes actuels Hutu et Tutsi ne sont pas les descendants de deux ethnies «pures» arrivées en bloc dans le pays. «Hutu» ne désignait aucune ethnie et le groupe actuel tutsi comprend beaucoup de gens qui ne sont pas des descendants du groupe ethnique tutsi d'origine» (Vansina J., 2000 b, p. 92).

J. Vansina se penche sur «l'origine» des Tutsi. Depuis 1950, on a abandonné l'idée que Tutsi et Hutu étaient des groupes «biologiques» différents. Mais 
comment expliquer leurs différences? Les premiers agriculteurs fondant et travaillant le fer et élevant un peu de bovins, qui vivaient dans la région de Butare, dès les premiers siècles avant J.-C., parlaientils le bantou parce que le mot Butare est un mot bantou signifiant «minerai de fer»? L'affirmation est bien téméraire! J. Vansina soutient l'idée que des groupes Tutsi sont arrivés depuis longtemps sur le territoire du Rwanda mais que leur système social et politique s'est constitué seulement au cours des quatre derniers siècles pour prendre sa forme actuelle au XIXe siècle. De toute façon, il ne faudrait pas confondre «l'origine» d'une catégorie sociale tutsi avec celle d'une population biologique tutsi. Y a-t-il un lien entre les Tutsi et le peuple nomade tuusi, qui vit dans le nord de la Tanzanie mêlé à des agriculteurs et parlant une langue sudnilotique? (Vansina J., 1993c).

La tendance dominante de nombreux auteurs, qui ont écrit sur le drame du Rwanda, a été d'en faire la conséquence d'une manipulation coloniale de l'ethnicité. Pour J. Vansina, le contexte a été plus complexe et les antagonismes sont plus anciens. La royauté de la seconde moitié du XIXe siècle (le Rwanda de Rwabugiri et des grandes familles de la Cour tutsi) a une responsabilité certaine dans les clivages sociaux, le culte de la violence, l'affirmation des identités physiques. Elle a failli à l'exigence du bien public et a créé des exploités, pasteurs et agriculteurs, ce qui devait à terme conduire à des révoltes (Vansina J., 2001b et 2004).

Voici une autre contribution à la critique des sources orales de l'histoire du Rwanda. D. Newbury rappelle le rôle des écrits de Marcel d'Hertefelt sur l'interprétation critique de ces sources et montre par exemple que les chronologies de quatre Etats voisins du Rwanda ont été déformées pour les mettre en accord avec la chronologie officielle rwandaise. II signale aussi qu'il n'a trouvé aucune trace d'une conquête militaire rwandaise du pays Shi, de Masisi ou d'Idjwi, au Kivu (Newbury D., 1993).

On a peut-être trop étudié l'histoire de la royauté rwandaise et peut-être trop peu celle des paysanneries rwandaises. Or les données qui ont été recueillies sur celle-ci sont nombreuses et de grande importance pour la compréhension des événements récents (Newbury C. \& D., 2000).

En 1933, A. Pagès avait attribué à ce qu'il appelait le royaume hamite du Rwanda une ascendance égyptienne. II avait mis l'accent sur une origine «égypto-pharaonique» des Tutsi. Un des arguments principaux était la présence de bovins à longues cornes en forme de lyre, semblables à ceux des bas-reliefs de l'Egypte antique. Manifestation d'égyptosophie? On comparera avec les thèses inverses que sont l'origine négro-africaine de la civilisation égyptienne et son corollaire, la Black Athena. Même type d'affabulation? (Sturtewagen C., 2006).

- Les études combinant sources orales et sources écrites

Venus d'abord en caravanes depuis le nord-ouest de l'actuelle Tanzanie pour le commerce du cuivre et de l'ivoire, les Yeke ont construit un royaume au Katanga (le chef le mieux connu a été Msiri). Une thèse leur est consacrée qui combine sources écrites et sources orales (Legros H., 1996 a). Soucieux de légitimer leur implantation au Katanga, les Yeke se sont incorporés dans l'univers mythique et rituel des autochtones en intégrant ceux-ci ainsi que les esclaves dans des rapports de parenté. Ils y ont réussi au point que l'imaginaire actuel des habitants du Katanga n'est pas loin de considérer les Yeke comme des pionniers de l'identité katangaise (Legros H., 1996 b). Ils ont joué un rôle de premier plan en 1960 dans l'expulsion des Luba du Kasai et dans la sécession katangaise, fournissant le ministre de l'intérieur du gouvernement Tshombe. Leur cas illustre bien comment a pu se constituer, s'enraciner et s'affirmer une ethnie, depuis les débuts de la période coloniale jusqu'à l'époque actuelle (Legros H., 1995).

Pour le Katanga également, et à peu près pour la même époque, voici deux sources, toutes deux orales à la base et transcrites au début du XXe siècle, l'une extraite du récit autobiographique de 
Tippo Tip, l'autre d'un rapport d'un administrateur de territoire, qui relatent le passage de Tippo Tip dans la chefferie luba de Mulongo (dans la partie nord de la dépression de l'Upemba) et le début de la pénétration arabe dans cette région (de Maret P. et Legros H., 1993).

Les autres aspects de l'histoire précoloniale

Les croisettes de cuivre sont un des témoignages archéologiques les plus connus du Katanga précolonial. P. de Maret en fournit une classification qui se fonde sur l'évolution de leurs formes. En remontant le temps: croisettes en $X$ et en barres du XXe au XVIIIe siècle, formes intermédiaires entre le $\mathrm{H}$ et le $X$ au sud et formes NN et $\mathrm{HX}$ au nord, du XVIle au XIIIe siècle, croisettes $\mathrm{HX}, \mathrm{HIH}$ et $\mathrm{HI}$ avant le XIVe siècle; enfin pour la période la plus ancienne (Ve -VIIe siècles) croisettes et lingots. La ressemblance de forme entre les croisettes et les lingots de métal des Phéniciens serait une simple manifestation de convergence. Les croisettes ont été tout à la fois «regalia, monnaie, insigne, moyen d'échange, objet d'art et matière première» (de Maret P., 1995)

A la fin du XVIe siècle, le petit Etat côtier de Ngoyo est un exemple intéressant d'Etat précolonial qui fut partenaire des commerçants portugais et hollandais puis courtier au moment de la traite atlantique. Connus pour leur activité de "caravaniers» (pourvoyeurs d'esclaves), les Woyo étaient aussi les gardiens des barracons où les esclaves étaient entassés avant leur embarquement. Ils ont fourni des matelots aux navires négriers. Le royaume déclina avec la fin de la traite (Mulinda Habi Buganza, 1993).

La traite des esclaves s'est maintenue sur la côte du Congo jusqu'au milieu du XIXe siècle sous l'appellation «émigration». Une relation du chirurgien Gaigneron, de la marine française, qui a visité différentes factoreries d'une maison de Marseille dans l'estuaire du Congo, en 1859, décrit le vaste barracon qu'était Saint Victor sur la péninsule de Banana où étaient rassemblés des esclaves destinés aux Antilles. Boma était encore un grand marché d'esclaves. Fait intéressant: Gaigne- ron y décrit des cas de maladie du sommeil. La maladie y serait apparue deux ans avant son arrivée et elle aurait pris, selon lui, une forme épidémique. C'est la première mention qui est faite de cette maladie pour cette région et pour le Congo (Salmon P., 1995 a).

Le royaume Kuba est le seul de tous les grands royaumes précoloniaux à avoir subsisté pendant la période coloniale. J. Vansina y voit trois facteurs explicatifs principaux: 1) la solidité de l'organisation politique centrale, 2) le caractère quasisacré du roi, 3) la réputation des arts kuba (Vansina J., 2007).

Un gros article étudie le rôle de la pirogue au milieu du XIXe siècle dans l'ouest du bassin du Congo. II défend I'hypothèse que la pirogue a été introduite à partir du nord (depuis les bassins du Nil et du Niger) dans le courant du premier millénaire avant J.-C., c'est-à-dire à un moment où se répand l'usage du fer, ce qui permet de creuser le tronc d'arbre qui donnera l'embarcation. L'article décrit les différents types de pirogues. Dans les eaux lentes et les marais, les pirogues étaient petites comme le libenge à fond plat de la Ngiri; sur les eaux profondes et rapides, les pirogues étaient longues et effilées. Les plus grandes pouvaient porter une centaine de personnes. Les pirogues servaient à la pêche et au transport. Elles étaient aussi utilisées dans les guerres entre populations riveraines. Au milieu du XIXe siècle, les grandes pirogues servaient au commerce de l'ivoire. Elles seront de moins en moins utilisées au XXe siècle sauf par quelques commerçants africains pour relier leurs établissements (Mumbanza mwa Bawele, 1997).

Des vestiges de villages entourés de fossés avaient été découverts dans les années vingt dans le Sud-Ubangi. Ces villages ont été abandonnés au milieu du XIXe siècle. On a émis diverses hypothèses sur les peuples qui les ont habités. Mumbanza mwa Bawele estime qu'il s'agissait de Bomboma-Likaw, venus de la rive droite de l'Ubangi, et de Bobo (Ngombe). Ils auraient été repoussés vers le sud par l'arrivée des Ngbandi, Mbanza et Ngbaka (Mumbanza mwa Bawele, 2003). 


\section{L'HISTOIRE COLONIALE PROPREMENT DITE}

Un livre accompagnant une grande exposition organisée au Musée de Tervuren sur la mémoire coloniale, dans le cadre du $175^{e}$ anniversaire de la Belgique, comporte une mosaïque de contributions (43 au total) qui en illustrent les multiples facettes ainsi que la variété des acteurs de toute origine. II comporte trois parties. La première est une interpellation sur des problèmes généraux, la deuxième analyse quelques thèmes regroupés en «pouvoirs, transactions, rencontres, savoirs, représentations» et se prolonge dans «le temps d'après» (l'époque postcoloniale). La troisième présente quelques individualités généralement peu connues, soit comme on les voyait dans les documents d'époque soit comme on les voit aujourd'hui. Les professeurs de géographie de l'enseignement secondaire trouveront, dans ce livre, de multiples illustrations et documents qui leur seront fort utiles (Vellut J.-L., 2005a).

\section{Les débuts de la période coloniale}

L'explorateur allemand Max Buchner a effectué de décembre 1878 jusqu'au mois d'août 1881, une expédition à caractère scientifique pour l'Afrikanische Gesellschaft in Deutschland (c'est-à-dire, en version complète, la Deutsche Gesellfschaft zur Erforschung Aequatorial Afrikas). II a traversé le pays lunda et séjourné pendant un semestre à la cour du Mwant Yav, grand chef des Lunda, à Mussumba. Un article résume son voyage et commente ses observations et ses remarques sur les peuples et les personnages qu'il a rencontrés. Celles-ci vont souvent à l'encontre des idées de son époque mais ne sont pas toujours exemptes de contradictions (Heintze B., 2004). Signalons que l'auteure de cet article a assuré aussi en 1999 la publication complète des articles, lettres et notes de l'explorateur.

Sur un personnage africain caractéristique des débuts de l'Etat indépendant du Congo, voici quelques pages qui résument la vie et les activités de Tippo Tip
(Huetz de Lemps C., 1994).

Nous avons rappelé, dans un bref article, les relations entre la géographie, les géographes et l'histoire du Congo, thème dont nous avions déjà abordé plusieurs aspects dans des articles antérieurs (Nicolaï H., 2007).

Pour susciter l'adhésion à ses entreprises africaines, Léopold II les a placées sous le couvert de ce que nous appelons aujourd'hui l'action humanitaire (à ce moment, la lutte contre la traite des esclaves) et de l'action scientifique (l'exploration géographique) et dans le cadre général de l'ouverture à la civilisation. II n'est donc pas étonnant de voir naître, en 1876, dans les mois qui encadrent la Conférence géographique qu'il réunit dans son palais de Bruxelles, les deux sociétés géographiques belges de Bruxelles et d'Anvers. Elles accompagnent l'action coloniale sans y participer directement. Celle de Bruxelles affirmera ses priorités scientifiques, celle d'Anvers visera plutôt la promotion des activités commerciales. Elles contribueront à rendre familière, à l'opinion publique, la présence belge en Afrique (Nicolaï $\mathrm{H}$., 1994).

Le Mouvement Géographique, par contre, fondé peu avant la Conférence de Berlin et dirigé pendant plus de 30 ans par A.-J-. Wauters, sera ouvertement un organe de propagande tout autant que de vulgarisation. II deviendra, après 1892, l'organe des sociétés belges au Congo. Si sa création a répondu très certainement à des sollicitations directes du Roi, il entrera en conflit avec lui au moment où Léopold II appliquera sa politique des grands domaines et des monopoles commerciaux (Nicolaï H., 1993 b). Un article analyse le contenu de ce périodique jusqu'en 1908 et montre la part prépondérante qu'y prennent le Congo et les activités des Belges dans ce territoire (Brugaillère M.C., 1993). Un autre s'intéresse plus particulièrement à son financement. L'essentiel de celui-ci est venu de la Compagnie du Congo pour le Commerce et l'Industrie. Mais Léopold II semble être intervenu directement dans le paiement du traitement de son directeur, ce que révèle un échange de lettres avec 
le secrétaire de l'E.I.C., au moment où le journal devient officiellement l'organe des sociétés belges au Congo. Le roi enjoint dans sa lettre de cesser ce paiement et d'en verser le montant à un journal d'Anvers pour qu'il soutienne sa politique. Jusqu'alors les contemporains considéraient le Mouvement comme l'organe officieux de l'E.I.C. (Henry E., 2008).

Si le Mouvement Géographique exprime les liens entre la géographie et le mouvement colonial, l'époque de sa publication (fin XIXe, début $X X^{e}$ siècle), a été aussi une période privilégiée pour les liens entre l'ethnographie (et l'ethnologie), la sociologie et le colonialisme. L'Institut de Sociologie Solvay, inauguré en 1902, crée en 1910, parmi ses groupes multidisciplinaires, un "groupe d'études coloniales» qui comprend plusieurs collaborateurs du Mouvement géographique. Ceux-ci répercutent dans le périodique le résultat de ses réunions. Ce groupe décida d'envoyer trois missions au Congo, plus précisément au Katanga. L'une étudiera les moyens de pénétration du commerce belge dans le Haut-Katanga et sera dirigée par un géographe économiste (Georges De Leener), la deuxième, dirigée par Georges Paquot, étudiera les possibilités d'établissement de colons belges et s'intéressera tout particulièrement à la question de la main-d'oeuvre dans ce pays peu peuplé (avec un recours éventuel à l'immigration chinoise). La troisième, confiée à l'ingénieur agronome A. Hock, étudiera la question agricole et son avenir. Mais aucune n'envisagera d'étudier systématiquement les populations et les sociétés autochtones (Crombois J.-F., 1998).

La colonisation du Congo a conduit à l'invention d'une vocation coloniale nationale et d'un pouvoir colonial doté d'une idéologie. Les sciences coloniales belges, qui en sont issues, ont été conçues à la fois comme la connaissance scientifique de l'Afrique et comme l'analyse du processus colonial. Toutes les institutions contemporaines belges liées à la connaissance des mondes lointains en sont dérivées (Poncelet M., 2008).

Quand le Musée de Tervuren fut fondé, en 1898, I'administration de I'E.I.C. était d'avis qu'il devait être consacré uniquement à un travail scientifique en évitant tout ce qui aurait pu avoir un aspect politique. Un ancien directeur de cette institution trace un tableau de l'évolution de la recherche qui s'y est faite. Dans les années 1920 encore, par exemple, la Bibliographie ethnographique, qui fut demandée par le Ministère des Colonies, devait rester de caractère documentaire et inventorial, à destination des fonctionnaires coloniaux, sans implication politique ou même sociologique. Mais l'ethnologie, la linguistique, la sociologie et I'histoire affirmèrent progressivement leurs champs de recherches et leur autonomie (Thys van den Audenaerde D., 2002).

L'histoire des frontières du Congo est d'un grand intérêt. Un article, auquel nous avions collaboré et dont nous parlons plus loin (Maboloko, 1999), insistait peutêtre trop lourdement sur l'idée que la frontière entre l'Angola et la province actuelle du Bandundu avait été tracée de façon totalement arbitraire sans aucun souci des organisations politiques territoriales et ethniques existantes. Elle comporte en effet beaucoup de tronçons utilisant des parallèles géographiques Nous laissions seulement entendre que le décrochement d'un degré vers le nord de sa partie orientale était lié à la prise en considération d'une de ces organisations. Un article décrit avec finesse la naissance de cette frontière. En 1885, l'empire lunda n'avait pas été incorporé au territoire de l'Etat indépendant du Congo sur les cartes de cet Etat. La frontière était fixée sur le $5^{\mathrm{e}}$ parallèle sud. En 1890, un décret annexa le Lunda tout entier. Le Portugal réagit. Un compromis résulta de pourparlers subtils entre Léopold II et le Portugal, avec intervention indirecte de l'Angleterre. Les petits royaumes reconnaissant l'autorité du Mwant Yav, souverain des Lunda, furent répartis entre le Congo et l'Angola. Comme il n'était pas possible de cartographier leurs limites avec précision, on les a, sur place, fixées sur des tronçons de cours d'eau et des parallèles. Les missions de délimitation (Grenfell pour I'E.I.C., Sarmento pour l'Angola) eurent à souffrir fortement de la faim dans ces 
régions du Kwango méridional dévastées alors par la disette (Vellut J.-C., 2006).

La frontière est du Congo, entre le lac Tanganyika et le lac Edouard est restée longtemps dans une certaine imprécision. En 1909 encore, les revendications des Anglais sur Rutshuru, avec l'accord des Allemands qui leur avaient donné une bande de territoire allant jusqu'à Goma, ont provoqué quelques incidents qui ont été finalement aplanis par un accord entre les trois puissances coloniales concernées, en mai 1910 (Lederer A., 1993).

Les frontières internationales du Katanga restent imprécises en de nombreux points: ligne médiane du lac Tanganyika, accès de Pweto au lac Moero, accès théorique mais inexistant en pratique au lac Bangweolo, etc.; seuls le méridien oriental et la crête Congo-Zambèze ont été bornés. Plusieurs groupes ethniques sont divisés entre plusieurs Etats et/ou deux provinces (Yemba P.T., 1999). Evidemment ce ne sont pas là constatations nouvelles.

Les Belbases, un fait frontalier particulier. A la suite de sa participation à la lutte contre les troupes allemandes de l'Afrique orientale, la Belgique a obtenu des facilités douanières de transit pour les marchandises en provenance ou à destination de ses territoires africains, aux deux extrémités du rail traversant le Tanganyika Territory, Dar-es-Salaam et Kigoma. Les équipements de ces zones portuaires, en somme des zones franches, ont été gérés par une société belge jusqu'en 1996 (Darcis L., 2007).

Parmi ses nombreuses études étymologiques (et toponymiques), souvent surprenantes, publiées dans Congo-Afrique ou dans les Annales Aequatoria, F. Bontinck se penche sur l'origine du mot mundele, au pluriel mindele, qui désigne le Blanc européen. Ce mot kongo est-il une référence à la couleur de la peau (mais dans la cuvette équatoriale, la langue populaire qualifie de rouges les Européens)? Une référence à un mot proche désignant la baleine (avec laquelle auraient été comparés les premiers navires européens)? Ou au fait que les blancs étaient toujours porteurs d'étoffes?
(Bontinck F., 1995b).

Dès l'époque de l'Etat Indépendant, des Congolais sont venus, à la suite de circonstances diverses, recevoir une formation scolaire en Belgique. Ce fut le cas d'un fonctionnaire de Boma, probablement originaire du pays yaka. Faut-il le considérer comme un précurseur de l'intelligentsia congolaise? La démarche est peut-être téméraire (Makamu, 2002).

Bien que l'Etat Indépendant eût érigé le franc comme monnaie officielle en 1887, différents objets importés ont servi longtemps de moyens d'échange: mitako (fils de cuivre ou de laiton), cauris, perles, tissus, couvertures, mouchoirs, etc. Les mitako ont été utilisés, même par l'Etat, jusqu'en 1912. Des croisettes de cuivre ont même été fabriquées en Belgique avec une contremarque officielle (Schoonheyt J., 2005).

Les transports au début de la colonisation Un fait anecdotique montre un aspect mineur des tentatives faites pour résoudre le problème du transport et de l'utilisation de l'énergie animale, l'acclimatation des chevaux de trait paraissant impossible en Afrique centrale. La mémoire populaire a conservé le souvenir de l'arrivée, en 1904, à Léopoldville, depuis Ténériffe, à l'initiative d'un Commissaire de District du Stanley Pool, d'une douzaine de dromadaires. Ils étaient une trentaine en 1910 après un deuxième arrivage et furent employés sur les chantiers de construction. On n'en parle plus après 1913 (Bontinck F., 1995a).

De 1882, lancement de l'En Avant, 5 tonnes, le navire de Stanley, à Léopoldville jusqu'à 1898, arrivée du chemin de fer dans ce poste, la navigation, en amont du Stanley Pool, se fit sur des bateaux de petites dimensions, dont les éléments devaient être acheminés par portage depuis Matadi. Les conditions de navigation étaient certes pittoresques mais souvent difficiles (Geladé V., 2002).

\section{Cent ans de présence missionnaire}

Le centième anniversaire de l'arrivée des missionnaires belges au Congo a été commémoré par la publication de plu- 
sieurs ouvrages et articles. Nous avions mentionné déjà, dans notre chronique précédente, celui des Scheutistes (Congrégation du Coeur Immaculé de Marie C.I.C.M.) arrivés les premiers (Bontinck F., 1988).

Les missionnaires jésuites sont arrivés en 1893 après que leur Compagnie eut refusé par deux fois les propositions de Léopold II. Comme la congrégation des Scheutistes, elle avait beaucoup hésité à aller travailler à côté d'Européens, aventuriers de toutes espèces, gens sans moeurs et sans foi, où les hérétiques (entendez les protestants) étaient nombreux et où gouvernement et administration risquaient d'être entre les mains d'une majorité de libéraux et même de francs-maçons! II semble que ce soit la proposition de confier aux religieux l'éducation d'enfants orphelins (ou libérés des esclavagistes), rassemblés dans de grands établissements qui devaient en faire des travailleurs et des soldats, qui emporta l'adhésion de la Compagnie. Elle y vit l'occasion d'en faire des chrétiens. Toutes ces argumentations sont reprises à peu près telles quelles, sans guère de commentaires critiques, dans les ouvrages commémoratifs. Citons d'abord une thèse d'histoire religieuse, soutenue à Rome en 1981, remaniée et mise à jour à cette occasion par L. de Saint Moulin (Mukoso N. F., 1993) et un article synthétique de L. de Saint Moulin (de Saint Moulin L., 1993).

Un livre rassemble les communications présentées lors d'un colloque commémoratif à Kimwenza en décembre 1993 (La Compagnie de Jésus..., 1993).Comme dans l'ouvrage précédent, il y est question, à plusieurs reprises, des fermes-chapelles, notamment dans la communication de L. de Saint Moulin sur le fondateur de la Mission et dans le discours de clôture de S. Decloux. Leur cas est intéressant pour les géographes car cette opération d'envergure avait, parmi ses objectifs, une transformation des campagnes congolaises. On sait que les Jésuites y rassemblèrent les enfants qui leur étaient confiés (ou fournis?) par l'Etat. Ces établissements, dirigés chacun par un catéchiste, étaient non seulement des écoles mais aussi des exploitations agricoles assurant la subsistance des élèves et vendant parfois des produits au dehors (caoutchouc provenant de la culture de lianes, par exemple, ou bien, le long du Kwango, bois pour les chaudières des bateaux de passage). Les fermes-chapelles disparurent après 1911, d'abord à la suite des critiques très sévères de la Commission d'enquête de 1904-1905 puis surtout à la suite du rapport du substitut Leclercq qui accusait les Jésuites d'avoir volé la plupart des enfants qui s'y trouvaient et de vouloir constituer au Congo des «réductions» comme au Paraguay. Les articles et ouvrages dont il est rendu compte ici reprennent les arguments qui ont été développés à l'époque et, sans nier totalement les faits reprochés, en atténuent souvent le caractère comme ils le firent il y a un siècle. II faut dire que, dans de tels ouvrages, qui célèbrent surtout les succès obtenus, il n'est pas d'usage que les points noirs soient mis en évidence. Le recrutement forcé des enfants et l'aide demandée par un missionnaire à la Force publique pour récupérer les enfants enfuis (avec même l'organisation d'une véritable expédition militaire) sont expliqués en partie par la nécessité du recours à la contrainte, un mal qui aurait été nécessaire à cette époque. La crise, qui éclata finalement entre les Jésuites et les autorités était en partie aussi le résultat d'un malentendu. Les autorités de l'Etat Indépendant fournissaient des enfants pour que les missionnaires les préparent à être des soldats et des fonctionnaires, et secondairement des ouvriers et des employés pour les sociétés coloniales. Les missionnaires voulaient former des communautés rurales chrétiennes et voulaient éviter que leurs membres partent ensuite dans ces lieux de perversion morale qu'étaient les nouveaux centres urbains. Autre remarque: à notre époque, où l'on stigmatise les mariages précoces et les mariages arrangés sinon forcés dans certaines communautés immigrées en Europe, serait-ce un anachronisme de s'étonner que les missionnaires mariaient, à 12 ans, dans des unions plus ou moins consenties, les filles dont ils avaient la charge, pour les garder du dévergondage? 
Une communication expose comment se fit l'extension de la Mission dans le Kwango, à la suite notamment d'accords avec les Huileries du Congo belge (groupe Lever) mais rien n'y est dit sur le comportement des missionnaires face aux abus commis par les sociétés huilières envers leurs coupeurs de fruits. II y est fait allusion dans d'autres publications, plus spécialement en ce qui concerne les réticences de certains missionnaires (mais les cas semblent avoir été rares) vis-à-vis des expropriations de fait des terres indigènes qui résultaient de l'application des "contrats tripartites» délimitant les terres concédées aux H.C.B. II fallut attendre aussi les années qui suivirent la Seconde Guerre mondiale pour que les Jésuites remettent en cause leurs réserves à l'égard des villes et de l'évolution sociale qui s'y produisait (L. de Saint Moulin, 1993, cité plus haut). Le livre commémoratif donne aussi de nombreuses informations sur les relations entre Jésuites et Scheutistes, notamment sur la façon dont s'est fait le partage de leurs territoires respectifs. La ligne de «démarcation» à Léopoldville fut la voie ferrée nord-sud jusqu'au Pool. On verra aussi comment un projet universitaire des Jésuites, sur leur site de Kimwenza, en somme dans le prolongement de leurs grands établissements d'enseignement du Bas-Congo, a été accaparé par I'Université de Louvain, donc par les autorités diocésaines belges, pour devenir I'Université Lovanium, dont les Jésuites furent, en partie au moins, écartés. On trouvera également, dans le même ouvrage, des informations sur la coopérative de Kisantu qui fut une grande opération de la fin de la période coloniale et sur des actions menées plus récemment par les Jésuites dans d'autres parties du Congo, notamment la prise en charge des enfants des rues à Bukavu après 1970.

La paroisse jésuite de Kimwenza, sur le site de l'Université de Kinshasa, a fêté son centenaire en 1993. Elle avait été créée comme "colonie scolaire» pour accueillir des enfants libérés des caravanes esclavagistes et qu'un décret avait confiés à la tutelle de l'Etat. II était convenu que les quatre cinquièmes d'entre eux seraient incorporés plus tard dans l'armée congolaise, le cinquième restant étant destiné à devenir des catéchistes et à former des ménages chrétiens. Mais la maladie du sommeil a décimé la colonie qui fut supprimée en 1899, les enfants étant renvoyés dans leur pays d'origine ou à Kisantu. Le poste est abandonné de 1901 à 1946. II est rouvert peu avant l'implantation de Lovanium. Les catéchistes formés à Kimwenza devinrent souvent des capitas de fermes-chapelles (Moya Mamonabwa H., 1993).

L'implantation des Jésuites au KwiluKwango est souvent décrite par eux comme une course contre les progrès des hérétiques (les protestants). II est intéressant d'entendre le point de vue de ceux-ci, particulièrement celui des missionnaires de l'American Baptist Foreign Mission Society. Les missionnaires baptistes eurent à se plaindre de la discrimination dont ils étaient l'objet par exemple pour la concession de terres, ou dans leur exclusion de l'enseignement dans les centres H.C.B. (pourtant société d'origine britannique), ou dans leurs difficultés à obtenir des subventions pour leur action médicale pourtant très importante. L'attitude des missionnaires catholiques à leur égard était même parfois très offensive. Ainsi en 1931, l'un de ceux-ci, après une exhortation digne de Pierre l'Ermite, entraîna les villageois de la région de Kingungi dans une véritable croisade pour reconquérir les villages gagnés au protestantisme pendant l'absence, pour des raisons diverses, de missionnaires jésuites depuis 1929. Les missionnaires catholiques réfutèrent cependant les allégations de certains journaux qui avaient accusé les missionnaires baptistes d'avoir encouragé la révolte des Pende (Smith R., 2003).

Plusieurs livres sont des éditions commentées d'écrits de missionnaires, dont des journaux, notamment de Fernand Allard, qui a travaillé dans les fermes-chapelles et d'Emeri Cambier, scheutiste au Kasai. Ils montrent bien les problèmes rencontrés au début de leur installation (Gallez D., 2001; Cornet A. et Bontinck F., 2001).

Le Père Cambier rêvait d'un royaume chrétien sur terre. On trouve ainsi, chez 
les missionnaires scheutistes, une version flamande, romantique et nationaliste, d'un christianisme colonial. Les missions ont été implantées à l'écart des postes de l'Etat car les missionnaires flamands auraient vu dans l'Etat (francophone) un instrument d'oppression culturelle du peuple mais aussi parce qu'ils ne voulaient pas que les Noirs chrétiens se comportassent comme les fonctionnaires. Par la suite cependant les missionnaires s'implanteront dans les centres d'activité de la vie coloniale, notamment le long du chemin de fer B.C.K. ainsi qu'à Bakwanga et à Tshikapa. L'article traitant ce sujet considère que, depuis l'action des missionnaires scheutistes, «l'espace du pensable sera désormais celui des convertis et baptisés [...] tandis que celui de l'impensable regroupera les non-baptisés [...] Les premiers sont élus et se veulent les modèles de la nouvelle société, tandis que les seconds seront refoulés..." (p.277). Les missionnaires blancs seront identifiés aux héros mythiques ancestraux (Kalulambi Pongo M., 1993).

Les violences dans l'Etat Indépendant de Léopold II

Il a été beaucoup question, au cours de la dernière décennie, de «mémoire coloniale» et particulièrement des crimes contre l'humanité commis pendant la période coloniale. Ceux de l'esclavage et de la traite des esclaves, qui ont fait l'objet de débats animés au sein d'enceintes internationales, sont antérieurs à l'action belge outre-mer. Mais ceux qui ont (ou auraient) été commis notamment dans l'Etat Indépendant du Congo, ont été rappelés et dénoncés avec virulence dans des ouvrages anglo-saxons qui ont ranimé ainsi la campagne qui avait été menée en Angleterre et aux Etats-Unis, au début du $X X$ e siècle. II s'agit là d'une question que les géographes ne peuvent ignorer en raison des séquelles que les événements évoqués ont pu laisser dans les sociétés africaines actuelles, par exemple, pour prendre un cas concret, dans leurs caractéristiques démographiques et les souvenirs qu'ils ont laissés dans la mémoire des villageois. Un journaliste américain, Adam Hochschild, leur a consacré un livre passionné qui s'appuie sur de très nombreuses sources dont évidemment le rapport du consul anglais Casement et celui de la Commission d'Enquête de 1904-1905, les écrits de E. Morel mais aussi les livres de D. Vangroenweghe et de J. Marchal. Ce livre est certainement un brillant travail de journaliste mais il n'apporte rien de vraiment nouveau sur les événements qu'il décrit. C'est surtout un réquisitoire qui reproche aussi aux milieux politiques et intellectuels belges d'avoir jeté volontairement un voile d'oubli sur ces événements (Hochschild A., 1998 et 2007).

Un film documentaire de Peter Bate (White King, Red Rubber, Black DeathRoi blanc, caoutchouc rouge, mort noire), réalisé pour la B.B.C. et fondé en partie sur ce livre, avec des interviews de quelques scientifiques belges, a été diffusé à la VRT et à la RTBF en avril 2004. Peu après cette diffusion, des contestataires flamands se sont attaqués à des monuments commémoratifs de cette période. La main d'un personnage africain du monument à la gloire de Léopold II sur la digue d'Ostende, a été symboliquement coupée (il a fallu trois jours pour qu'on s'en aperçût et encore à la suite d'une information communiquée à la presse par les auteurs de l'opération!). Une statue de Baudouin, en 2005, à Ostende, à l'occasion de l'anniversaire de l'assassinat de Lumumba et, en 2008, celle de Léopold II à Bruxelles, ont été peintes en rouge. Des menaces ont visé le monument De Bruyne-Lippens à Blankenberghe et la statue du Général Jacques à Dixmude (Meeuwis M., 2005). Cependant l'Arcade du Cinquantenaire, qui aurait dû avoir la charge symbolique la plus forte dans ce domaine, car elle était, pour Emile Vandervelde, l'arcade des mains coupées, construite avec l'argent du caoutchouc rouge, a été épargnée jusqu'à présent.

A côté d'approbations enthousiastes, le livre de Hochschild et le film ont nourri une polémique qui s'est exprimée dans de nombreuses notes et articles. Citons à titre d'exemple, une note de A. Knockaert dans Congo-Afrique, qui reprend notam- 
ment les critiques de Barbara Emerson, un commentaire de J. Stengers dans un entretien publié par Le Soir du 13 octobre 1998, "Entre le coup de poing et la caricature» et, dans le livre sur "La mémoire $d u$ Congo", la mise au point de Philippe Marechal sur "La controverse sur Léopold II et le Congo dans la littérature et les médias» (Marechal P., 2005). Beaucoup de journaux et périodiques belges, anglais, américains, français et même japonais ont repris, le plus souvent sans nuances, les accusations de Hochschild, allant jusqu'à utiliser le terme de génocide. L'exposition de Tervuren elle-même a provoqué de nouveaux soubresauts comme ceux qui ont eu lieu, on l'a vu plus haut, à Ostende. Elle a entraîné une forte réaction de Hochschild luimême, dans The New York Review of Books (In the Heart of Darkness, October 6, 2005). II a vu, dans l'exposition, une tentative de réfutation de son livre et s'est indigné qu'on y ait donné une estimation aussi basse que $20 \%$ pour la diminution de la population congolaise pendant la période léopoldienne (les auteurs avaient repris pourtant la propre estimation d'E. Morel, une des sources de Hochschild, dans sa préface au pamphlet de Mark Twain contre Léopold II, pamphlet qui a été réédité en français en 2004). Dans un échange de lettres avec J-L. Vellut, dans le New York Review of Books du 12 janvier 2006, Hochschild se défend d'avoir parlé de génocide, contrairement à certains de ses lecteurs, mais estime que le mot holocauste est tout à fait adéquat. On remarquera cependant que le terme «holocauste» disparaît du sous-titre de la version française en format de poche publiée en 2007. Le sous-titre «Un holocauste oublié" de l'édition 1998 y est remplacé par "La terreur coloniale dans l'État du Congo 1884-1908», plus conforme au sous-titre de la version originale. Dans un épilogue inédit, l'auteur donne son avis sur les réactions que son livre a suscitées en Belgique (Hochschild A., 2007).

Un fonctionnaire territorial dont la carrière a été interrompue par l'indépendance du Congo et s'est poursuivie par une carrière de conseiller technique puis de diplo- mate, est devenu un pourfendeur de l'action coloniale belge et particulièrement de l'action des sociétés privées. Après plusieurs livres passablement virulents, publiés en néerlandais, sous le pseudonyme d'A.M. Delathuy, sur la Commission de 1904-1905 et sur les missionnaires au Congo (Pères Blancs, scheutistes, jésuites, rédemptoristes, trappistes, norbertins, etc.), il a publié en français, depuis 1996, cinq livres, deux sur les exactions de l'Etat Indépendant du Congo et trois sur le travail forcé (pour les mines de cuivre et d'or, pour la construction des chemins de fer, pour la production d'huile de palme). Ces livres, dont plusieurs ont été largement utilisés par $\mathrm{A}$. Hochschild, contiennent un grand nombre de données nouvelles, provenant d'archives du ministère des Affaires Etrangères, habituellement inaccessibles mais que, sans doute par son appartenance à l'administration diplomatique, l'auteur a pu consulter. On peut se demander cependant si certaines ont été soumises à une critique historique suffisante (Marchal J., 1996a, 1996b, 1999, 2000, 2001)

J. Vansina, dans l'interview recensée plus haut (Vansina J., 2001, Forum, n²1, p. 17) s'interrogeant sur l'ampleur de l'indignation manifestée par J. Marchal (et aussi par Vangroenweghe), tout particulièrement à l'égard de Léopold II, suggère qu'elle était peut-être dirigée surtout contre la haute bourgeoisie francophone de Bruxelles: «vooral ging over de hogere franstalige burgerij van Brussel en dat hun anti-nationale inquisitie vooral ook daartegen gericht was» (p. 17). Pour eux, cette haute bourgeoisie aurait dirigé toute l'action coloniale belge.

D'autres publications découvrent (ou redécouvrent) ou apportent des éléments nouveaux sur le recours à la violence pendant l'ère coloniale, surtout au début de celle-ci. Un colloque entier lui a été consacré. Est-ce à dire que l'époque antérieure a été idyllique? Pour J.-L. Vellut, avant la pénétration coloniale proprement dite, les zones de violence se trouvaient dans l'intérieur ravagé par les incursions esclavagistes venant des régions côtières plus stables de 
I'Atlantique ou de l'Océan Indien. L'occupation coloniale du Congo a commencé avec Stanley, qui était enclin à la conquête par la violence. Cette violence s'est ensuite amplifiée, à partir de 18901891, quand l'Etat puis les sociétés coloniales ont adopté le modèle swahili d'exploitation, remplaçant l'esclavage inclus dans ce système par la fourniture forcée de caoutchouc. II faut cependant se méfier de la «légende noire» qui entoure l'histoire du Congo. Elle a connu une recrudescence quand, dans un raccourci imprudent et suite à une lecture erronée du roman de Joseph Conrad, dont elle avait négligé l'aspect exotique et dont elle ne s'était pas rendu compte qu'il décrivait un Congo antérieur au développement de la cueillette du caoutchouc, Hannah Arendt a établi une filiation directe entre le colonialisme de Léopold II et les régimes totalitaristes nazi et stalinien et l'a décrit ainsi comme un précurseur de l'holocauste des années 40. La thèse a été reprise et amplifiée par Adam Hochschild et différents médias (Vellut J.-L., 2004).

P. Salmon, dans des carnets tenus par des fonctionnaires ou des commerçants de l'Etat Indépendant, livre des témoignages accablants sur la facilité avec laquelle on recourait à la violence pour obtenir la soumission des villageois. La litanie des villages brûlés et des rafales de coups de feu tirées dans la brousse ou la forêt, où pouvaient se cacher des villageois insoumis, laisse pantois. Tous ces carnets nous éclairent sur les comportements des acteurs de cette époque et plus particulièrement sur leurs relations avec les Africains (Salmon P., 2004).

On remarquera, en tout cas, que la «repentance» qui s'est manifestée au cours des deux dernières décennies à propos du passé colonial belge, explique sans doute l'absence de toute commémoration ou même de toute allusion officielle ou dans le médias du centième anniversaire de la reprise du Congo par la Belgique. Seul un colloque, très discret, en 2009 a discuté la figure et les actions de Léopold II. Triomphe du politiquement correct actuel?

Sur la continuité dans la pratique de la violence, sous le régime Mobutu, voir
Sabakinu Kivilu, 2004.

Le Territoire de Banzyville a souffert de l'exploitation du caoutchouc à partir de 1900. Une zone y avait été érigée en domaine privé de l'Etat. La contrainte a été ici d'autant plus forte que les savanes prédominent et que les ressources forestières en caoutchouc ont été vite épuisées. De nombreux habitants ont fui les opérations militaires en gagnant la rive française. La cueillette du caoutchouc a certainement favorisé la propagation de la maladie du sommeil (Ngbwapkwa Te M., 2003).

La période du Congo belge (1908-1960)

Pierre Ryckmans a été incontestablement une des grandes figures de l'histoire coloniale belge. J. Vanderlinden lui consacre une imposante biographie où se trouve notamment exaltée son activité pendant la deuxième guerre mondiale (Vanderlinden J., 1994). On lira, à ce sujet, les réflexions de B.Verhaegen, d'une part sur les risques que comporte la méthode biographique et d'autre part, sur l'absence d'allusion (et d'explication) dans ce texte à ce qu'il considère comme la position immobiliste de Ryckmans, pendant sa carrière de gouverneur général, vis-à-vis d'une émancipation politique des Congolais. J. Stengers objecte sur ce dernier sujet que Ryckmans ne disposait pas, dans la politique coloniale, d'une autonomie suffisante et qu'il faut mettre à son actif, pendant la guerre, d'avoir su contenir la revendication des colons belges qui réclamaient une participation à la gestion politique de la colonie (Verhaegen B., 1995).

On lira aussi avec intérêt la correspondance privée de Pierre Ryckmans, futur gouverneur général, sur la façon dont se faisait le recrutement de la main-d'oeuvre dans les districts des H.C.B. (Huileries du Congo Belge). II avait été chargé d'une mission sur ce sujet en 1930-1931, dans le Congo-Kasai. Le ton est assez différent sans doute de celui du rapport qu'il a rédigé. On y trouvera notamment une critique des données démographiques disponibles à l'époque. A de nombreuses reprises, Ryckmans fait des tentatives 
infructueuses pour calculer la population des différents territoires qu'il a parcourus et par conséquent la main-d'oeuvre qui y était «recrutable» en confrontant des estimations qu'il fait lui-même et les chiffres des documents officiels, On y trouve aussi des descriptions très vivantes des lieux et des personnages (européens surtout) (Vanderlinden J., 2007).

C'est quelques mois après la mission de P. Ryckmans qu'éclate, dans le sud du territoire de Kikwit et dans le nord de celui de Kandale, en partie à cause des mesures de contrainte qui y étaient pratiquées et du comportement des agents des sociétés huilières, la révolte des Pende, qui fut une des plus graves, sinon la plus grave, de l'histoire du Congo belge. Un mémoire rappelle ses causes et, grâce à l'utilisation d'archives conservées à Bruxelles, et du rapport d'enquête Jungers, décrit en détail et de façon critique les opérations militaires ainsi que la répression qui suivit. Rappelons au lecteur qu'une thèse d'histoire avait été soutenue sur cette révolte par Sikitele Gize a Sutumbula à l'Université de Lubumbashi en 1986 mais qu'elle n'avait pu disposer de ces documents et reposait en grande partie sur les souvenirs conservés par la population locale (Vanderstraeten J., 2001). Sur le rôle de la Force Publique dans le maintien de l'ordre au Congo belge, vu notamment du côté africain, voir Vellut J.-L. (1994)

L'interview de vieux villageois, entre 1966 et 1986, dans les Territoires de Bulungu et de Masi Manimba et le dépouillement des archives de ces territoires, montrent quels ont été les rapports des chefs (médaillés, coutumiers, de secteurs non coutumiers) avec l'administration coloniale, tiraillés entre les exigences des autorités belges et les demandes des villageois. Si quelques points positifs de l'époque coloniale apparaissent dans leurs propos (comme le fait qu'on pouvait voyager d'un village à l'autre sans être tué ou enlevé comme esclave, ou bien la fin des guerres entre villages), leurs témoignages sont une liste de contraintes imposées à la population pour la participation au recensement, la fourniture de caoutchouc dans le cadre de l'effort de guerre (1940-1944), la construction des routes, la construction des bâtiments publics, le regroupement et le déplacement des villages dans le cadre de la lutte contre la maladie du sommeil, le recrutement pour la reconstruction du chemin de fer Matadi-Léopoldville, le recrutement pour la Force Publique, une liste aussi d'exactions commises par les policiers, et parfois par les agents territoriaux européens eux-mêmes, d'interventions de ces derniers pour empêcher les événements de la vie villageoise (danses qualifiées d'obscènes, cérémonies diverses) qui auraient empêché le travail dans les palmeraies, du recours à la chicotte (jusqu'en 1959), etc. (Smith R. E., 2005). Les cas cités sont nombreux mais leur fréquence réelle ne peut être précisée. Dans le Kwilu également voici une contribution mineure à l'histoire de la géographie administrative: la constitution du Territoire d'Idiofa, dans l'actuelle province de Bandundu (Mufogoto G., 2007).

La crise économique des années trente, par suite de la chute des prix offerts aux villageois et de la pression exercée par les société coloniales, a été une des causes indirectes de la révolte des Pende. Les conséquences de la crise pour les villageois des différentes régions de l'Afrique centrale ont varié selon le degré d'encadrement colonial. Dans le Haut-Ogooué (Moyen Congo, Congo français), le faible encadrement administratif contraignait les autorités à passer par l'intermédiaire des chefs africains pour percevoir l'impôt et accroître la production et donc à user de palabres et d'encouragements verbaux. Comme les chefs ne pouvaient pas trop demander à leurs dépendants, le supplément de production exigé n'avait pas d'implication importante sur la production de subsistance. Par contre, dans le territoire de Lisala (Congo belge), l'imposition ou le renforcement de la culture obligatoire de coton, en 1933, perturba davantage l'économie de subsistance notamment par ses implications sur le calendrier agricole, qui entraîna aussi un recours plus important à la culture du manioc et par conséquent l'aggravation des carences alimentaires (Driesmans I., 2002). 
La crise de 1930 a eu aussi des conséquences importantes sur les structures économiques et administratives du Congo. Les structures administratives sont remaniées en 1934-1935 et se maintiendront ensuite jusqu'à l'indépendance. La crise a accentué les monopoles notamment les monopoles régionaux des grandes sociétés coloniales (groupe Empain au Kivu, Société Générale au Katanga et au Kasai, Cominière en Equateur, etc.), a renforcé la voie nationale, a entraîné la création de l'INEAC et l'apparition des premiers centres de recherches (ou d'application) liés à des université belges (Tshibangu Kabet Musas, 1995).

Après 1925 et surtout après 1928, I'Union Minière du Haut-Katanga, en vue de stabiliser sa main-d'oeuvre, a encouragé l'installation de femmes dans les camps de travailleurs pour que se constituent des ménages. Elle payait les dots prévues par la coutume mais, comme elle voulait des femmes résistantes, elle alla jusqu'à intervenir dans le choix des épouses. Celles de moins de $45 \mathrm{~kg}$ et celles ayant un petit bassin, par exemple, étaient écartées. L'Union Minière voulait que ces femmes soient aussi des partenaires économiques, en cultivant un jardin vivrier (parfois sous la contrainte), en même temps qu'un élément de reproduction de la force de travail (avec des encouragements comme des primes de naissance, des cadeaux aux bébés, etc.). La société minière entra en conflit avec les sociétés cotonnières parce que la vente du coton permettait aux polygames villageois de se procurer plus facilement des épouses supplémentaires et de faire concurrence ainsi aux jeunes travailleurs U.M.H.K. Elle prit prétexte de la lutte contre la polygamie pour combattre cette concurrence (Dibwe dia Mwembu, 1993). L'effondrement de la Gécamines après 1990 a marqué la fin d'une longue histoire sociale qui avait conduit à la formation de familles ouvrières au Katanga. Celle-ci est analysée par un historien issu de ces familles. II montre que cet effondrement a laissé beaucoup d'“orphelins» à la fois orphelins de la société mourante et orphelins de leurs familles et de leurs clans d'origine. Un degré supplémentaire dans cet arrachement a été atteint avec le cas des familles originaires du Kasai qui ont été chassées du Katanga (voir plus loin le problème des «refoulés») (Dibwe dia Mwembu D., 2001).

La dernière décennie de la période coloniale fut marquée par l'application d'un «Plan décennal pour le développement économique et social du Congo belge» publié en 1949. D'une ampleur considérable, ce plan d'investissements publics a été financé uniquement par les ressources propres de la Colonie (l'Etat belge intervint seulement pour garantir les emprunts que le Congo dut contracter sur le marché international). Un mémoire analyse sa genèse, l'esprit dans lequel il fut conçu, l'accueil qui lui fut réservé, les lacunes qu'il comporta (la part des investissements pour le développement de l'agriculture traditionnelle a été tout à fait insuffisante contrairement à l'objectif proclamé de sortir de l'économie de subsistance), la façon dont il fut réalisé (20 $\%$ à peine des investissements du programme routier par exemple furent exécutés) et montre que ce Plan, loin de modifier les structures extraverties de l'économie congolaise, les a renforcées.

Un énorme travail (trois volumes, un total de plus de 1200 pages) a été consacré à l'histoire du rail au Congo. Réalisé par des passionnés de la question, il comporte une iconographie d'une telle abondance qu'un lecteur un peu moins passionné risque de s'y perdre (Blanchart C. et al., 1993, 1999 et 2008).

La production de cacao au Congo belge fut précoce mais resta peu importante. L'État Indépendant avait décidé de la développer autour de ses stations. C'est au Mayumbe, à la fin du XIXe siècle, qu'apparurent les premières plantations privées dans un système qui associait le Roi, des familles de la noblesse belge, les missionnaires scheutistes et quelques colons indépendants, avec une maind'oeuvre africaine quelque peu forcée. Les plantations villageoises préconisées par I'INEAC après 1935, dans le cadre des cultures imposées, puis par le Plan décennal, n'eurent pas un grand succès (Vellut J.-L., 1996b; sur le cas du Mayumbe, voir Vantieghem L., 1996). 


\section{Autres aspects de la société coloniale}

La «ménagère», c'est-à-dire la concubine africaine de l'homme européen, a été une institution de toute une époque de la colonisation. Plusieurs publications lui ont été consacrées (Lauro A., 2005).

Entre les deux guerres mondiales, les autorités coloniales avaient confié un rôle "civilisateur» aux femmes européennes (religieuses, infirmières, épouses de fonctionnaires ou de cadres d'entreprises) surtout à l'égard des femmes africaines. L'épouse devait aussi soutenir le moral (et le comportement moral) du mari. Des femmes européennes se sont investies dans la protection de l'enfance et la promotion de la femme africaine. L'article étudie le rôle de certaines d'entre elles, dont Jeanne Rogissart qui, arrivée avant la première guerre mondiale, milita pour la création d'un enseignement pour les filles afin qu'elles puissent devenir les épouses des jeunes Africains instruits. Elle aura peu de succès (Jacques $\mathrm{C}$. et Piette V., 2003).

Sur la répartition des rôles entre hommes et femmes par le pouvoir colonial, on lira la belle synthèse de Nancy Rose Hunt (2005). Une thèse de doctorat a été défendue à l'UCL sur le rôle que les femmes (religieuses, femmes d'administrateurs coloniaux, infirmières africaines, etc.) ont joué au Rwanda, pendant la période d'entre deux guerres, dans le fonctionnement des systèmes de santé et parfois aussi dans un objectif de contrôle social (Cornet A., 2005).

Le cas des mulâtres a posé un problème délicat à la société coloniale. Le thème était assez largement tabou. Voici, sur ce sujet, un livre et un article (Jeurissen L., 2002 et 2003). Le cas des «mulâtres», issus d'un blanc et de sa ménagère et abandonnés par leur père à son départ, n'avait pas été envisagé dans le cadre législatif qui ne distinguait que deux catégories, les indigènes (c'est-à-dire les Africains) et les non-indigènes (les blancs). Certains, comme le $\mathrm{Dr}$ Dryepondt, en 1923, étaient d'avis qu'il fallait les laisser se fondre peu à peu dans la masse indigène pour éviter la création d'une «caste» qui haïrait et envierait les
Européens, dont ils n'auraient ni l'autorité ni les droits et qui mépriserait profondément les noirs et s'en ferait détester. En 1938, la Commission permanente pour la Protection des Indigènes préconisait qu'un enfant mulâtre reconnu par un Européen fût éduqué dans la société métropolitaine. II n'y eut que quelques interventions ponctuelles de l'administration coloniale dans cette voie (création d'orphelinats, par exemple).

Sur la façon dont le droit colonial belge a intégré ou non les mécanismes juridiques précoloniaux, on lira une importante communication de J. Vanderlinden (2004).

En ce qui concerne le rôle des minorités ethniques dans les villes d'Afrique centrale, voir le résumé d'une thèse soutenue à l'université de Gand, en 2006, sur l'histoire sociale des communautés islamiques d'Usumbura (Castryck G., 2007).

Sur l'émergence des «évolués» et sur leur rôle dans le développement du nationalisme congolais dans la dernière décennie coloniale, on lira l'ouvrage très fouillé de Mutamba Matombo (1998). C'est un travail indispensable pour comprendre la façon dont s'est faite une décolonisation qui a pesé d'un poids essentiel sur la vie ultérieure de la population congolaise.

L'Eglise catholique a encadré beaucoup d'associations d'évolués, comme celle des anciens élèves des Pères de Scheut, l'ADAPES, qui fut au départ liée à l'Action catholique. Elle visait la formation d'une élite intellectuelle chrétienne qui devait constituer «le levain dans la pâte» (Tshimanga C., 2001a). Sur les réticences des autorités coloniales à former une élite, sur l'ambiguïté de leur position à cet égard et sur les maigres résultats obtenus, on lira Thsimanga C. (2001b).

La question de la nationalité, qui s'est posée notamment à propos des mulâtres et des «évolués» est restée très confuse pendant toute la période coloniale. Aucun texte officiel ne l'avait définie explicitement. Pourtant on considérait que la nationalité belge s'appliquait aux habitants noirs du Congo belge. Mais elle était purement théorique et ne les protégeait pas des mesures de ségrégation raciale et ne leur donnait pas de toute façon les 
droits des citoyens belges. Les Congolais étaient des sujets belges. Cependant le Parlement belge n'a pas jugé contradictoire ou inutile d'en naturaliser quelquesuns dont un employé du Musée de Tervuren (De Meester T., 1998). Les questions de nationalité ne disparaîtront pas avec l'indépendance. C'est la nationalité congolaise qui désormais sera en cause. Elle sera source de graves conflits.

\section{La naissance des Eglises africaines}

La période coloniale a vu l'apparition d'Eglises congolaises que l'on peut considérer, dans une certaine mesure, comme une réappropriation africaine des Eglises chrétiennes. Ces religions nouvelles procèdent-elles aussi des cultes traditionnels? Une étude en fait l'hypothèse et estime qu'il y a eu un processus de transformation des cultes qu'elle appelle «territoriaux» vers les églises chrétiennes congolaises modernes. Elle concerne la partie du Bas-Congo qui se trouve au nord de Boma. Après une période d'hostilité ouverte envers les missionnaires, les Kongo ont compris qu'il fallait rechercher des voies nouvelles pour faire face aux défis du colonialisme et reprendre le contrôle des relations entre leur société et le monde surnaturel. Comme ils ont considéré que les missionnaires apparaissaient plus efficaces que leurs propres prêtres dans la relation avec les divinités (et ce qu'on attendait de cellesci comme faire tomber la pluie par exemple), ils ont décidé d'intégrer des éléments de la religion des missionnaires dans leurs propres cultes. Un fait à noter: certains de leurs sites sacrés sont devenus des lieux de pèlerinage chrétiens (Vanhee H., 1998).

J.-L. Vellut, en utilisant des documents contemporains de la prédication de Simon Kimbangu, montre que sa personnalité et sa trajectoire s'inscrivent dans une modernité, qui cherche à récupérer les symboles d'un Occident hégémonique. II donne une interprétation approfondie des réactions des missionnaires protestants et catholiques, du pouvoir colonial et des milieux coloniaux. On lira, à la fin de son article, la lettre du substitut du Procureur Général «intègre mais pas téméraire» qui demande la grâce royale pour Kimbangu, qu'une cour militaire vient de condamner à mort, et qui l'obtiendra (Vellut J.-L., 1996). J.-L. Vellut a édité, dans la collection Fontes Historiae Africae, les sources relatives à Simon Kimbangu que l'on trouve dans les archives des missionnaires protestants du Bas-Congo, plus particulièrement les missions suédoises dont il était issu (Vellut J.-L., 2005). II décrit dans l'introduction générale l'itinéraire du prophète (pp. IX-XXVI). La position des milieux catholiques à l'égard de Kimbangu est restée très hostile, même lorsque l'administration coloniale eut adouci la sienne, et cette hostilité se maintiendra par la suite dans le Congo indépendant. Après le décès de Kimbangu, ils firent même circuler l'information (fausse) qu'il s'était converti au catholicisme à l'article de la mort. Cette position se greffait sur l'hostilité vis-à-vis des protestants dont il a été question plus haut (Zana Aziza Etambala M., 2004).

L'Eglise kimbanguiste annonce l'instauration du Royaume de Dieu sur Terre dans lequel le peuple kongo aura un rôle central de même que Nkamba, lieu de naissance du prophète, ville sainte, nouvelle Jérusalem. Depuis 1969, l'Eglise kimbanguiste, qui avait officiellement cessé d'être interdite par le pouvoir colonial deux années avant l'indépendance du Congo, a obtenu son adhésion, sous conditions, au Conseil Oecuménique des Églises. Elle avait dû pour cela élaborer une théologie plus orthodoxe. Cependant, vis-à-vis du peuple, elle conserve une théologie où la race noire, l'ethnie kongo et la personne de Kimbangu et de ses successeurs trouvent leur place dans une filiation avec les récits de la Bible. Dans les années 70 et 80, le mouvement a connu un renouveau spirituel avec l'apparition de groupes de prières. Les ambitions déclarées d'universalisme n'ont pas fait disparaître un ancrage dans l'ethnicité. Le peuple kongo doit mener le monde vers le Royaume des cieux (Mélice A., 2001). Depuis la mort, en 1992, de Joseph Diangienda, fils de Simon Kimbangu, le mouvement qui 
connaissait depuis longtemps des forces centrifuges, a connu aussi des tentatives de décentralisation et même un véritable schisme provoqué par une partie des petits-fils du fondateur, particulièrement ceux qui résident à l'étranger, dans la diaspora. Un nouveau site sacré, Monkoto, ancienne résidence de Diangienda à Kinshasa, concurrence même aujourd'hui celui de Nkamba (Mélice A., 2006).

Sur un autre mouvement prophétique apparu lui aussi en pays Kongo, à la fin de l'entre-deux-guerres, "la Mission des Noirs", issue de l'Armée du Salut, voir l'article de Zana Etambala (2005)

En pays Zande, dans le nord-est du Congo, une Eglise chrétienne syncrétique "Mission ti Africa MTA», née dans les années 40 avec un culte marial prononcé, rechristianisée par son fondateur dans les années 50, compte des prophétesses aussi bien que des prophètes (Grootaers J.L., 1999).

Sur la situation actuelle des Églises chrétiennes africaines, on consultera aussi, dans cette chronique, le chapitre consacré à la géographie des villes congolaises puisque c'est là que l'effervescence religieuse se manifeste le plus vigoureusement.

L'enseignement, les associations pour la jeunesse, les nouveaux groupes sociaux

A la fin de la période coloniale, on a souvent soutenu l'idée que les élèves des missions protestantes recevaient une formation inférieure à celle des élèves des écoles catholiques parce que les autorités belges avaient une attitude discriminatoire envers l'enseignement protestant. On peut se demander si cette prétendue infériorité n'était pas aussi (et principalement) le résultat de l'application d'un modèle éducatif mis au point aux EtatsUnis peu après la Guerre de Sécession, pour les Noirs américains (un des foyers de ce modèle a été l'Institut normal et professionnel de Tuskegee, en Alabama). Conçu, comme on le disait peu élégamment, pour assurer l'instruction des «arriérés ethniques, Nègres et PeauxRouges", ce modèle visait l'acquisition d'une formation uniquement profession- nelle (n'atteignant que rarement le niveau supérieur) et religieuse (Serufuri Kakiza P., 2004).

Les manuels en usage dans les écoles congolaises, écrits dans les langues locales, nous donnent d'intéressants reflets des préjugés et des comportements des Blancs et des Noirs. Outre le fait qu'ils constituent une contribution élémentaire à la naissance d'une littérature en langue locale et qu'ils sont aussi l'expression de certaines idées pédagogiques, ces manuels ont un contenu idéologique et politique qui mérite d'être analysé. Le centre Aequatoria de Bamanya (Mbandaka) a publié dans ses Annales Aequatoria de nombreux articles sur le sujet, généralement sous la plume de $\mathrm{H}$. Vink. Celui qui est publié dans le Bulletin de l'ARSOM, compare deux manuels destinés aux enfants Mongo. L'un, rédigé (vers 1920?) par un missionnaire, l'évêque de la Nouvelle-Anvers et de Lisala, décrit les bienfaits de la colonisation. L'autre, écrit vers 1937, est l'oeuvre d'un enseignant africain, fortement inspiré par un missionnaire farouchement critique de la colonisation. Le premier dit, par exemple, qu'avant l'arrivée des Blancs, les Africains mouraient comme des mouches, l'autre, qu'il n'y avait pas de maladies graves et que ce sont les Blancs qui les ont apportées et disséminées dans le pays. Le premier affirme que, selon la Bible, toute autorité provient de Dieu. Mobutu, qui avait probablement utilisé ce manuel, a pu rappeler ainsi dans une interview accordée à une journaliste sud-africaine en 1997, que, si Dieu ne l'avait pas voulu, il n'aurait pu devenir président du Zaïre (Vink H., 2000 b). Sur les interférences entre le Congo et l'enseignement tant en Belgique qu'au Congo, on lira le petit texte écrit à l'occasion d'une exposition sur ce thème à Ypres (Depaepe M., 1994).

Le scoutisme, par ses origines et son idéologie, s'inscrit, constate J.-L. Vellut, dans les histoires impérialistes de l'époque. Malgré diverses réticences, les missionnaires l'ont introduit au Congo dès 1920, les Pères Blancs à Mpala, sur le lac Tanganyika puis les Scheutistes à Léopoldville en 1922. Ils y ont vu assez 
vite un instrument pour l'émergence d'une élite chrétienne. Les trois premiers prêtres congolais ont été scouts. Ce fut le cas du futur cardinal Malula. Les principaux auteurs du manifeste de Conscience Africaine (1956) étaient d'anciens scouts ainsi que le tiers des délégués à la Table Ronde (Tshimanga C., 1999). L'implantation du scoutisme en général (vers 1930, les effectifs des scouts congolais, essentiellement africains, représentaient près du cinquième des effectifs des fédérations catholiques belges) et surtout son implantation à Elisabethville sont étudiées aussi par Tilman S. (2001).

Le rôle du football dans l'émergence d'une nouvelle société congolaise est décrit par Van Peel B. (2001). D'abord cantonné à la société blanche, il en a accentué le quadrillage en identités sociales (Léo-Est plus populaire, Léo-Ouest plus bourgeois) ou nationales (Belges, Portugais, Léo contre Brazza, etc.). Il a contribué ensuite à diversifier les identités africaines elles-mêmes. II a été utilisé lui aussi comme instrument missionnaire par l'Eglise catholique. On rappellera que le premier très grand stade de Léopoldville, le stade Roi Baudouin, fut l'oeuvre du missionnaire scheutiste Raphaël de la Kethulle de Ryhove. II fut rebaptisé stade Tata Raphaël après l'indépendance avant de devenir le stade du 20 mai. Fait à noter mais d'interprétation ambiguë: le règlement des matches entre équipes africaines stipulait que les joueurs devaient jouer sans souliers. Sur le développement du football à Léopoldville, voir aussi Mumbanza mwa Bawele (2008). L'auteur constate que très peu d'encouragements ont concerné les sports «nationaux», comme par exemple la lutte, telle qu'elle était pratiquée chez les groupes de la cuvette. P. Salmon rappelle les graves incidents qui se produisirent à l'issue du match entre I'Union Saint-Gillois et une équipe africaine le 16 juin 1957 quand des émeutiers lapidèrent les voitures des Européens après le match qui avait vu la victoire de l'équipe saint-gilloise. Un prélude aux émeutes de janvier 1959 (Salmon P., 1992). Nous pourrions ajouter qu'en 1959, c'est à l'occasion d'un match à Luluabourg que se produisirent les premiers affrontements violents entre Lulua et
Luba. Sur le développement des activités sportives, de certains aspects physiques du scoutisme, au Rwanda, à la fin de la période coloniale et leur contrôle par l'Etat et les missions catholiques, avec la prise en compte des clivages ethniques, on lira Riot T. (2008).

Sur les différents aspects de la culture à l'époque coloniale, un colloque a été organisé à Kinshasa en 2004 par le Centre Wallonie-Bruxelles. Les communications ont été publiées dans la revue CongoMeuse (volumes 6,7; 8,9) (Quaeghebeur M. et Bibiane Tshibiola, 2007 et 2008). On lira notamment la contribution de $\mathrm{S}$. Cornelis sur les arts visuels.

Dès l'époque de l'Etat indépendant du Congo, des artistes belges ont été engagés pour accompagner des expéditions et en rapporter des documents iconographiques ou bien ont été chargés directement de telles missions. Nous disposons ainsi de tableaux de sites, de localités et de paysages, de scènes de la vie rurale et de la vie urbaine qui sont souvent d'un grand intérêt historique et géographique en plus de leur valeur artistique. Une exposition leur a été consacrée à Woluwe-Saint-Pierre en 2003. Le beau livre, qui l'a accompagnée, en contient de nombreuses reproductions. Certains de ces artistes ont continué en Europe à être inspirés par leur séjour au Congo, d'autres y sont retournés pour d'autres oeuvres de commande (Guisset J., 2003).

\section{LA DÉCOLONISATION ET LES DÉBUTS DE LA RÉPUBLIQUE DU CONGO}

\section{Les acteurs belges}

La réédition de l'entrevue accordée en 1980 par J.J. Van Bilsen, qui fut l'auteur, en décembre 1955, d'un Plan de trente ans pour l'émancipation politique de l'Afrique belge, puis conseiller du président Kasavubu et enfin cheville ouvrière de la Coopération belge outre-mer, permet de se rendre compte combien l'establishment politique et académique belge était peu préparé à envisager l'indépendance au milieu des années 50. D'où l'atmosphère de panique et de précipitation dans laquel- 
le celle-ci se fit en 1960. (Blondeel W., Foutry F. et Van Mensel F., 2000). Le rôle et la vision de J.J. Van Bilsen sont décrits dans un article qui accompagne cette réédition (Beke D., 2000).

Il est intéressant de connaître les idées de L. Pétillon qui était gouverneur général au cours des années qui ont précédé le processus vers l'indépendance, donc au moment de la déclaration de J.J. Van Bilsen (Stenmans A. et Reyntjens F., 1993). D'un point de vue anecdotique, on lira les impressions peu optimistes du Commandant de la gendarmerie nationale de Belgique, le lieutenant-général Godfroid, venu au Congo en 1953 pour étudier le projet de création d'une gendarmerie congolaise, projet qui n'eut pas de suite (Salmon P., 1995 c).

On lira aussi les témoignages qu'apportent les notes et les récits d'André Ryckmans, fils de l'ancien gouverneur général, qui fut jeune administrateur territorial dans les années qui précédèrent l'indépendance, à qui l'on doit notamment des rapports dépourvus de préjugés sur le mouvement kimbanguiste et qui essaya vainement de convaincre ses supérieurs d'amorcer plus rapidement la décolonisation de l'administration. On sait qu'il fut assassiné en 1960 par des soldats de la Force Publique alors qu'il négociait la libération de prisonniers belges (Ryckmans G., 1995).

Y a-t-il eu un modèle belge de décolonisation? Dans un colloque consacré aux décolonisations, Stengers J. (1995) discute la question. II est intéressant de comparer l'évolution des deux Congos, belge et français, de 1956 à 1965 (Le Callenec S., 1995). Dans l'ouvrage qui contient ces deux articles, les cas du Rwanda et du Burundi sont également traités (Chrétien J.-P. et Gahama J., 1995). Lire aussi pour le Congo le point de vue de Vellut J.-L. (2000).

\section{Les acteurs congolais}

Voici une grosse étude sur l'ABAKO, le premier parti politique congolais à avoir vu le jour. Conçu d'abord au départ, en 1950, comme une association de défense de la langue et de la culture kongo, il affirma explicitement ses revendications poli- tiques dans un manifeste d'août 1956. En atténuant les thèses autonomistes de ses débuts, il joua un rôle essentiel dans les négociations qui virent la naissance du Congo indépendant et, avec Kasavubu, fournit le premier président de la république (Verhaegen B., 2003).

On aura une idée de la rapidité de l'évolution des idées dans la population congolaise elle-même en comparant le comportement public et les écrits de Patrice Lumumba de 1950 à 1958, quand il glorifie encore l'apport de la colonisation belge et l'action du roi bâtisseur, et son discours du 30 juin 1960 (Verhaegen B., 1993). Voir aussi, sur ce sujet, un mémoire du même auteur et de J. Omasombo (Omasombo Tshonda J. et Verhaegen B., 1998).

Sur Patrice Lumumba, personnage tragique de l'indépendance congolaise, de nombreux livres ont été publiés au cours de ces quinze dernières années. Une commission parlementaire d'enquête a même travaillé sur les circonstances de son assassinat, à la suite de la publication d'un ouvrage d'un journaliste dénonçant l'implication directe ou indirecte d'acteurs belges et même de personnalités politiques importantes. Un ouvrage, publié dans la série des Cahiers africains, traite de l'action de Lumumba dans une phase cruciale des négociations pour l'indépendance (Omasombo J. et Verhaegen B., 2005). Un historien congolais vient de décrire toute cette période et celle qui a suivi (Zana Aziza Etambala, 2008).

On relira avec intérêt un livre, publié en anglais au début des années 1960 et dont la traduction française a été assurée en 1994. En décrivant la naissance d'un parti à caractère non ethnique, le Parti Solidaire Africain, il montre les mécanismes qui ont pu réaliser brièvement, sur le terrain et dans l'action, l'union de populations rurales et d'un petit groupe d'intellectuels idéalistes (et sans doute un peu naïfs) (Weiss H., 1994).

C'est sous la direction d'un des dirigeants de ce parti, Pierre Mulele, que se développa, au Kwilu, de septembre1963 à la mi1966, une rébellion, qui a eu, dans quelques-uns de ses aspects, l'allure d'une jacquerie en ce sens qu'elle expri- 
mait les ressentiments d'une population paysanne contre les profiteurs de l'indépendance (hommes politiques nationaux et nouvelle bourgeoisie congolaise). Elle se produisit en pays pende et mbunda et renoua ainsi avec la tradition d'insubordination qui s'était manifestée, trente ans plus tôt, par la révolte des Pende, mais en y ajoutant un habillage marxiste-léniniste et certains aspects de la pensée révolutionnaire chinoise et algérienne. Voici un livre sur le personnage tragique que fut Mulele et sur la spécificité de sa révolution (Verhaegen B., 2006).

Un épisode curieux des débuts des premières années de l'indépendance illustre bien les mythes et fantasmes qu'a toujours nourris le Congo aussi bien en Europe que dans d'autres parties du monde, et les dangers de certaines idées géopolitiques. II s'agit de la malheureuse expédition entreprise par une des icônes tiers-mondistes pour exporter la révolution cubaine au Congo. Après l'échec de la révolution de Stanleyville, Ernest Che Guevara crut que les montagnes du Kivu, au-dessus du lac Tanganyika, à la latitude de Fizi, allaient lui permettre de reproduire le succès de la guérilla de la Sierra Mæstra et qu'il pourrait y entraîner, dans le mouvement, les populations locales. II arriva avec un contingent de 120 soldats cubains, tous noirs, à l'exception du médecin. Croyance naïve dans la vocation des montagnes à être des foyers victorieux de rébellion? Méconnaissance totale de la géographie aussi bien physique qu'humaine du Congo? Pourquoi aller faire le Tarzan en Afrique? lui avait pourtant dit Ben Bella qu'il avait rencontré peu avant. L'opération reçut l'appui - mais un appui très mou - de Laurent Kabila dont le propre maquis était installé dans ces lieux. Les populations locales sont restées indifférentes. Sept mois plus tard, après divers fiascos dont une tentative avortée de s'emparer d'Albertville, l'expédition retraversa le lac Tanganyika. Le monde ne fut guère averti de ces événements. Fait curieux: il semble que la CIA n'eut pas connaissance de la présence de Guevara. Celui-ci fit une opération semblable peu après, avec les mêmes erreurs "géopolitiques» et, cette fois, de façon définitivement tragique, en
Bolivie (Verhaegen B., 2000).

Kabila, dont le maquis était installé dans cette région, a fait sa réapparition publique, quand il a été propulsé à la tête de la coalition anti-mobutiste en 1996. On lira avec intérêt l'avant-propos de J.-L. Vellut à un mémoire de licence de I'Université de Kisangani consacré au maquis Kabila. Celui-ci avait pu maintenir, dans les montagnes au-dessus de Fizi, un îlot de résistance de caractère socialiste, sans doute en partie grâce à une certaine connivence avec des forces militaires zaïroises locales, connivence matérialisée vraisemblablement par une participation commune à un trafic d'or (or artisanal ou or prélevé sur la production des sociétés minières). Un avant-poste perdu des affrontements entre opposants de la guerre froide par Africains interposés (Wilungula B. Cosma, 1997)! Un essai biographique a été consacré au personnage de Kabila (Kennes E., 2003). Voir aussi Willame J.-C. (1999).

\section{Le départ de la population européenne}

Ce départ a été d'un grand poids dans l'évolution du pays après l'accès à l'indépendance. Cette population était cependant de toute façon d'installation temporaire car il n'y avait pas eu de colonisation de peuplement et l'on n'avait jamais encouragé l'installation de «petits blancs». Au 1er janvier 1958, il y avait 109400 Européens dont 86700 Belges, pour la plupart fonctionnaires, missionnaires et agents de sociétés coloniales. II n'y avait que 5200 «colons» belges c'est-à-dire établis à leur compte personnel. Au Ruanda-Urundi, en 1960, la population européenne était de 7976 personnes dont 6064 Belges. II y eut plusieurs vagues de retours. La plus importante se place en 1960 (44 484 rapatriés en juillet), puis il y eut 1963 (fin de la sécession katangaise), 1964 (séquelles de la rébellion de Stanleyville), 1973 (zaïrianisation des entreprises étrangères), 1978 (événements de Kolwezi), 1990 (fin de l'assistance technique belge), septembreoctobre 1991 et janvier 1993 (pillages à Kinshasa). En février 1993, il ne reste plus officiellement que 2800 Belges au CongoZaïre (dont 600 à Kinshasa). Par contre on 
évalue, en 1990, à 20000 les Zaïrois installés en Belgique (dont 12000 officiellement). L'article qui traite ces questions envisage aussi comment s'est faite la réinsertion des rapatriés en Belgique ou dans d'autres pays (Salmon P., 1995 b).

Les différences d'évolution politique du Rwanda et du Burundi

Jusqu'au début des années 1990, en simplifiant, on pourrait dire qu'au Rwanda, où les revendications politiques ont été exprimées en termes ethniques dès les années 50 (sur cette question voir aussi le livre de Daley P. (2006), le pouvoir politique a été accaparé par la majorité ethnique (hutu) tandis qu'au Burundi, où ces revendications ont été plus tardives et où une tentative de coup d'Etat hutu avait échoué, il l'était par la minorité (tutsi). Fort logiquement, le maintien de la situation avait engendré plus de violence au Burundi qu'au Rwanda et il avait fallu y imaginer des procédures pour protéger la minorité au pouvoir (Reyntjens F., 1993). Au Burundi, les manifestations de nature folklorique ont même été l'objet de manipulations politiques et sociales qui ont renforcé les identités ethniques et par conséquent la division nationale, aboutissant ainsi à l'échec des tentatives de démocratisation et de multipartisme. Les affrontements entre une armée à dominance tutsi et les populations paysannes hutu (200 000 morts en 1972!), entraînèrent le départ de réfugiés vers le Rwanda et contribuèrent par là à y favoriser les réactions anti-tutsi (KadendeKaiser R.M., Kaiser P.D., 1997)

\section{LES ÉVÉNEMENTS DES DEUX DER- NIĖRES DÉCENNIES}

Les événements politiques, qui se sont produits en Afrique centrale depuis la date de notre dernière recension, ont donné naissance à une multitude de publications surtout à partir de l'implosion qu'a été la catastrophe rwandaise. II est malaisé de choisir ce que pourrait retenir le géographe dans cette masse où historiens, anthropologues politiques, spécialistes de sciences politiques et aussi journalistes et moralistes ont apporté chacun leur témoi- gnage, leur point de vue, leur explication. Deux groupes de publications sont à distinguer. II y a eu d'abord, c'est-à-dire par ordre chronologique, les commentaires sur l'évolution politique du Zaïre de Mobutu suite à la constitution de la Conférence nationale souveraine dans ce qu'on espérait être une transition vers la démocratie. II y a eu ensuite tout ce qui a été écrit sur les tragédies du Burundi et du Rwanda et sur leurs répercussions dans l'est du Congo et finalement dans le Congo tout entier, c'està-dire la conquête du pays par l'Alliance des Forces Démocratiques pour la Libération du Congo, la fin du régime Mobutu, la prise de pouvoir de Kabila et tous les soubresauts qui ont suivi dont ce que certains ont pu considérer comme la première guerre continentale africaine mettant en scène le Congo, le Rwanda, I'Uganda, l'Angola et le Zimbabwe et, de façon indirecte, le Burundi et la République centrafricaine.

\section{L'évolution politique du Congo}

- Les pillages de 1991 et 1993

Du premier groupe, nous signalerons d'abord quelques articles sur les violences et les pillages qui se sont produits à Kinshasa en 1991 et 1993 (Ndaywel è Nziem, 1998; Devisch R., 1998), publiés dans la revue française Cahiers d'Etudes Africaines.

\section{- La période de transition}

Dans la série des Cahiers Africains, G. de Villers s'est intéressé tout particulièrement aux relations entre les événements politiques au Congo-Zaïre et la Belgique (de Villers G., 1994 et 1995) puis il a décrit les différentes phases d'une transition chaotique et peu efficace vers la démocratie (instauration du multipartisme à la fin 1990, ouverture officielle d'une Conférence nationale souveraine en août 1991 qui adopte en 1992 une nouvelle Constitution, puis multiples péripéties). La prise de pouvoir de Kabila, en mai 1997, a mis fin à cette période (de Villers G. et Omasombo Tshonda J., 1997). Sur les événements qui se sont produits entre cet épisode et la guerre de 1998 (tentative rwandaise d'é- 
carter Kabila), voir le livre de de Villers G. et Willame J.-C. (1998). Sur ce qui a suivi la guerre de 1998 jusqu'à l'assassinat de Laurent Kabila, voir de Villers G. (2001). Sur l'arrivée de Joseph Kabila au pouvoir, les amorces de guerre civile, les négociations entre fractions concernées sous couverture internationale, les élections et les débuts de la Troisième République, G. de Villers publiera un gros livre récapitulatif en 2009 (de Villers G., 2009). Sur la façon dont se sont déroulées les négociations entre fractions congolaises et sur leurs résultats, voir Willame J.-C. (2002) et aussi Bouvier P. (2004).

Bien sûr, beaucoup d'articles publiés dans les deux premiers tiers des années 1990 ont été assez vite dépassés par les événements. Ils contiennent pourtant des éléments d'analyse intéressants sur différentes questions (la nationalité, la pression ethno-régionaliste, etc.). Ils jettent aussi quelques lueurs sur les conditions de vie des populations bousculées par ces événements, notamment les fonctionnaires et salariés qui ont vu disparaître leurs sources de revenus. Ainsi dans l'article d'Obotela R.N. (1997), on lira, ce qui avait déjà été évoqué par Ngondo a Pitshandenge, que, dans l'incapacité de payer leur loyer, des enseignants des écoles primaires de Kinshasa, font camper leur famille dans la cour de leur école et la nuit occupent les salles de classe. Même comportement pour des membres du personnel administratif de l'Université qui occupent les sous-sols des amphithéâtres avec femmes, enfants et même petit bétail.

La revue Afrique contemporaine (Documentation française), consacre un dossier spécial au thème "Du Zaïre au Congo» (Gaud M., 1997a). On y trouvera notamment une chronologie politique (Gaud M., 1997b), une comparaison avec le précédent ougandais, un article de géopolitique de Pourtier R. (1997) sur un territoire en quête d'Etat, un article sur la façon dont la presse a traité la question des réfugiés, un article sur le thème un peu éculé du «scandale géologique».

Chevauchant les thèmes de l'évolution du Congo et les événements de la région des Grands Lacs, signalons aussi la vue d'ensemble que donne un petit livre écrit de façon assez alerte par deux journalistes sur la géopolitique du Congo (Cros M.-F. et Misser F., 2000). Cet aperçu présente les qualités mais évidemment aussi les défauts des ouvrages de ce type où la pertinence de l'information peut être très variable selon la période ou le thème. Notons par exemple que le poids de la population rurale, qui reste la population majoritaire du pays, et celui de toutes les activités traditionnelles et modernes du milieu rural sont quasi absents (deux pages au maximum) de ce tableau géopolitique.

Dans les dernières années du régime mobutiste, les symboles de l'Etat zaïrois perdent toute leur force. La dénomination «Zaïre» a été immédiatement contestée. La Conférence Nationale Souveraine avait proposé «République fédérale du Congo». Le pouvoir en place ne l'accepta pas mais on se mit à utiliser l'expression CongoZaïre ou Zaïre-Congo. Dans un article qui est plutôt en faveur du maintien de la dénomination «Zaïre» parce qu'elle était une création qui prétendait symboliser la «supratribalité» de la nation, en remarquant toutefois que, lors de son adoption, les grandes sociétés congolaises ne l'avaient pas toutes introduite dans leur nom en remplacement de la référence au Congo (la Gecomin était devenue la Gecamines et l'Otraco, l'Onatra), I'historien Ndaywel propose un nouvel hymne national et prend position en faveur du retour de l'étoile jaune sur fond bleu ciel dans le drapeau national (Ndaywel è Nziem I., 1996). On est revenu aussi aux noms anciens des provinces (Bas-Congo, Province Orientale, Katanga). En 1998, la revue Afrique-Zaïre reprend son titre Congo-Afrique et publie ( $n^{\circ}$ 324, pp. 238-239) le texte du décret-loi de 1997 sur l'actualisation des dénominations des entités et autorités administratives. Elle publie aussi un résumé des actes d'une Conférence sur la Territoriale, qui décrit tous les fléaux qui ont frappé l'administration territoriale depuis l'indépendance (irresponsabilité, improductivité, tribalisme, népotisme, clientélisme, manque d'esprit d'initiative et de créativité, trafic d'influence, corruption, affairisme, tracasseries administratives et policières, arbitraire, etc.) (Conférence..., 1998). Etudiant le rôle du sentiment ethnique 
dans les conflits de la RDC, L. de Saint Moulin estime qu'un facteur dont il faut tenir compte aussi, est l'inégale répartition de la population urbaine et celle des investissements. Elles seraient à l'avantage du sud-ouest et du sud-est par rapport au nord du pays. L'insuccès relatif des rébellions dans le sud s'expliquerait, selon lui, par cette donnée fondamentale de la géographie humaine (de Saint Moulin L., 2002)

- Les «refoulés», une forme d'épuration ethnique pendant la période de transition

Le régime mobutiste a commencé à trébucher en 1990 avec des manifestations d'étudiants à Lubumbashi qui ont été réprimées violemment par les autorités ce qui a entraîné la rupture de l'assistance technique belge. Peu après, des pillages commis en partie par les soldats suivis par la population ont frappé Kinshasa à deux reprises. Au Shaba-Katanga, la crise économique et politique a provoqué un phénomène qui a répété celui qui s'était produit lors de la sécession katangaise en 1960, c'est-à-dire le «refoulement», souvent violent, de populations d'immigration intérieure, en l'occurrence les gens originaires du Kasai. On estime que, de 1992 à 1994, entre 600000 et 800000 personnes ont dû fuir le Katanga et retourner dans leurs anciennes provinces. Cette «épuration ethnique» aurait fait de 50000 à 100000 morts. Cet exode perturba la vie économique et sociale du Katanga. Au Kasai, la plus grande partie des refoulés s'établirent dans la ville de Mbuji Mayi et dans le pays rural de Kabinda (Pourtier R., 1998). Dans la seule ville de Kananga, au Kasai occidental, entre octobre 1992 et août 1993, on a enregistré 70717 Kasaiens refoulés, soit, avec tous ceux qui n'ont pas été enregistrés, un total de l'ordre de 100000 personnes (Luishiye, 1993). Sur ce problème, voir aussi le livre de Bakajika B. (1997). En 2005, un professeur de Lubumbashi proposera, dans un gros article, une "relecture» de l'histoire des rapports entre les Katangais et les Kasaïens du Katanga (Dibwe Dia Mwembu D., 2005). Voici aussi sur cette question le témoignage et le point de vue d'un journaliste (Kabongo Malu E., 2007).

La tragédie rwandaise et ses répercussions

\section{- Les événements du Rwanda et du} Burundi

Sur ceux-ci et leurs conséquences, la littérature est mondiale et ne peut être détaillée ici. II faut peut-être rappeler qu'avant que ne se produise la catastrophe, l'Université Nationale du Rwanda (campus de Ruhengeri) avait publié un rapport de huit universitaires destiné explicitement à combattre «la campagne internationale menée par les mouvements de réfugiés tutsi» (Bangamwambo F.X. et al., 1991). Un document à verser au dossier des perceptions hutu. Sur tout ce qui s'est passé ensuite, il faut consulter la source précieuse qu'est l'Annuaire de l'Afrique des Grands Lacs, publié de façon récurrente depuis l'année 1996, par le Centre d'étude de la région des Grands Lacs d'Afrique (Rijksuniversitair Centrum Antwerpen), sous la direction de $S$. Marysse et F. Reyntjens (et aussi de S. Vandeginste). II permet de suivre les événements dans leur succession chronologique mais traite aussi certains sujets fondamentaux. Un document irremplaçable est la vaste enquête menée par l'organisme international Human Rights Watch où sont consignés les crimes contre l'humanité commis par toutes les parties en présence (Des Forges A., 1999).

On lira ainsi les ouvrages de Chrétien J.-P. (1995, 1997, 2000, 2002, 2003, 2007), Guichaoua A. (1995, 2004, 2005), Lemarchand R. (1996, 2002 a et b), Prunier G. (1995, 1997, 2006), Reyntjens F. (1994, 1995, 1997, 1999) et Willame J.-C. (1995, 1996, 1997 a, 1997 b) et différents articles de Pourtier R. (1996, 1997, 1998). Les uns traitent spécifiquement du Rwanda, d'autres du Burundi, d'autres de l'ensemble des trois pays concernés. Deux ouvrages de Chrétien J.-P. ou dirigés par lui $(1997,2002)$ traitent plus précisément du thème de l'ethnie et de l'ethnisme pour lequel nous avons déjà donné plus haut les avis de J. Vansina, de Salmon et 
d'autres auteurs comme L. de Saint Moulin. Lemarchand R. (2002a) rappelle le précédent des massacres de 1972 au Burundi (des Hutu par des Tutsi). On ajoutera sur tous ces événements la version discordante et outrancière de B. Lugan, connu pour des positions antérieures du même type. Elle conteste les thèses officielles du nouveau Rwanda en accusant même son président de ne pas avoir cherché à empêcher le massacre des Tutsi de l'intérieur parce qu'ils n'avaient pas participé à la rébellion et qu'ils pouvaient concurrencer, pour l'accès à la terre, les Tutsi revenant de I'Uganda (Lugan B., 2007). Récemment également $A$. Guichaoua a publié les résultats de la grosse enquête qu'il avait menée dans la préfecture de Butare, une préfecture très peuplée du sud du Rwanda où la population tutsi était proportionnellement plus importante que dans d'autres régions du pays et où Tutsi et Hutu étaient fort imbriqués et même unis par de nombreux métissages (Guichaoua A., 2005). Des politologues rwandais ont apporté leur contribution (par exemple Kimonyo J.-P., 2008). Certains articles évoquent l'idée que les interventions du nouveau gouvernement rwandais dans les territoires congolais voisins, pendant certains épisodes des conflits, pourraient annoncer des revendications territoriales (par exemple Newbury D., 1997). Des bibliographies critiques des événements ont été publiées dans diverses revues africanistes comme par exemple par Jefrenovas $\mathrm{V}$. (2000). On ne peut ignorer non plus divers livres écrits par des journalistes, qui ont certes été composés dans le feu des événements, mais qui contiennent de nombreux témoignages (par exemple Braeckman C., 1994). Signalons, dans cette catégorie, un ouvrage collectif auquel ont collaboré différents journalistes belges de la presse quotidienne et de la radio-télévision et des spécialistes en sciences politiques (cités dans plusieurs de nos références) sur les événements qui ont conduit Laurent-Désiré Kabila au pouvoir (Braeckman C. et al., 1998). Dans le $n^{\circ}$ 53 de Politique Africaine (1995), on lira notamment l'article de G.de Villers sur l'«africanisme» belge confronté à l'inter- prétation de la tragédie rwandaise (de Villers G., 1995). Voir aussi les points de vue des géographes Gotanègre J.-F., 1996; Imbs F., Bart F. et A., 1994; Imbs F., 1997, ainsi que ceux de Lanotte O., 2003; Mamdani, 2001; Mathieu P. et Willame J.C., 1998; Olson J., 1995.

Diverses revues ont consacré à ces événements des numéros entiers. La revue Les Temps Modernes a publié un gros volume sur les politiques de la haine (Les Temps Modernes, 1995). Dans un numéro triple des Cahiers d'Etudes Africaines consacré aux "Disciplines et déchirures. Les formes de la violence» (1998), on compte cinq articles relatifs aux événements du Rwanda, du Burundi et du Congo (Kivu et Kinshasa), notamment un article de $\mathrm{Cl}$. Vidal qui s'interroge sur le rôle des paysans pendant le génocide au Rwanda, un article de B. Ndarishikanye sur les rapports entre l'Etat et les paysanneries dans le conflit ethnique du Burundi, un article de P. Mathieu et A. Mafikiri Tsongo sur les guerres paysannes au Nord-Kivu, un article de I. Ndaywel è Nziem sur le lien entre les rébellions et les pillages, ces derniers faisant l'objet de réflexions de R. Devisch. D'un point de vue plus spécifiquement géographique, on citera un numéro double de la revue Hérodote intitulé Géopolitique d'une Afrique médiane (86-87, $3^{\mathrm{e}}$ et $4^{\mathrm{e}}$ trimestre 1997), avec des contributions de $R$. Pourtier, G. Prunier, F. Reyntjens, J.-C. Willame, J.H. Bradel et A. Guitaert, F. Imbs et $A$. Bart. Pour les trois derniers auteurs, qui sont des géographes et qui ne semblent pas, dans leurs travaux antérieurs, s'être rendus compte de l'imminence de la catastrophe, l'enjeu du conflit serait territorial, la croissance démographique rendant impossible le partage. Une combinaison de facteurs sociaux, économiques et politiques aurait provoqué l'explosion.

L'article de Françoise Imbs est particulièrement intéressant parce qu'il essaie, deux à trois années à peine après le drame, de dresser un bilan démographique et un bilan économique. Des cartes montrent l'ampleur de la diminution de la population d'avril à novembre 1994, l'évolution de la population des diffé- 
rentes préfectures d'avril 1994 à juin 1997. En avril 1994, la population du Rwanda était estimée à 7740000 habitants, elle était tombée en août 1995 à 5 millions (compte tenu du retour des Tutsi vivant à l'étranger). L'estimation des morts fluctue entre 500000 (chiffres de l'ONU, en trois mois, mais pour le seul génocide) et 850000 ( 1 million selon les nouvelles autorités gouvernementales). Les pertes les plus fortes sont dans le sud, dans la préfecture de Butare où elles auraient concerné $24 \%$ de la population. Les cartes montrent que le pourcentage des morts est proportionnel à la part des Tutsi dénombrés en 1991. La diminution de la population due aux morts et à la fuite de familles hutu a été compensée par le retour de quelques centaines de milliers de Tutsi (d'Uganda, du Burundi, du Congo, de la diaspora). Comme les hommes ont été tués, ou se sont enfuis ou ont été emprisonnés en plus grand nombre que les femmes, celles-ci faisaient en 1996 les deux tiers des actifs dans les campagnes où leur charge dans les travaux agricoles se trouvait fortement alourdie. Avec les retours massifs de 1996 depuis le Congo, la Tanzanie et le Burundi et malgré le fait que 280000 réfugiés ne sont pas revenus du Congo, la population en juin 1997 a rattrapé et aurait même dépassé de $3 \%$ son niveau d'avril 1994! Elle serait de 8 millions. Les mesures prises par les autorités pour regrouper une partie au moins de la population rentrée au pays dans des imidugugu (habitat regroupé) vont-elles modifier le paysage rural en dégageant de l'espace pour les cultures et en améliorant la desserte en équipements collectifs? On a réactualisé ainsi un vieux projet de l'époque coloniale. D'innombrables questions sont posées par l'auteur dont nous ignorons encore aujourd'hui les réponses. C'est que, après la profusion des travaux sur les événements des années 1994 à 1996, travaux produits surtout d'ailleurs par des politologues, nous n'avons pas jusqu'à présent de publications sur l'évolution qui s'est produite dans les campagnes, en dehors des rapports à caractère général des grands organismes internationaux. Le numéro
111 de la revue Hérodote revient en 2003 sur les Tragédies africaines. Dans la liste des publications géographiques proprement dites, signalons que nous avons nous-même traité des composantes démographiques du drame rwandais (Nicolaï H., 1997), esquissé ses conséquences pour le Kivu, dans un mémoire consacré à la répartition et à la densité de la population dans cette région (Nicolaï H., 1998a) et tenté une brève synthèse des relations entre la géographie, la démographie et la violence pour une revue italienne (Nicolaï H., 1998b).

Des médecins ont donné aussi leur avis sur les événements. $H$. Vis rappelle qu'il avait annoncé un effondrement (un collapsus) du Rwanda dans un avenir proche (vers 1995) si des mesures n'étaient pas prises pour contrôler la croissance de la population et combattre la malnutrition (voir nos comptes rendus de ses publications dans nos chroniques antérieures par exemple dans Nicolaï $\mathrm{H}$. et al., 1996, p. 267). Pour le Kivu d'altitude, le pronostic était identique mais à une échéance un peu plus lointaine. Les chercheurs avaient bien défini tous les problèmes et souvent proposé des solutions mais les autorités n'ont pas pris à temps les mesures nécessaires malgré les symptômes qui auraient dû les alerter, comme le développement de la disette dans le sud-est du Rwanda à la fin des années quatre-vingt rendant nécessaire le recours récurrent à l'aide alimentaire internationale. Les événements politiques (les attaques des Tutsi revenant de l'Uganda, dans le nord du pays provoquant la fuite de milliers de réfugiés) ont précipité le cours des choses (Vis $\mathrm{H}$. et Goyens P., 1996). Les mêmes auteurs avaient exposé leurs vues sur ces questions dans un article de la revue Lancet (Vis H. et al., 1994) et reviennent sur le sujet dans un article de 1997 (Vis H., 1997b).

Une dizaine d'années après le drame rwandais, on commentera sa signification à l'occasion de sa commémoration officielle et notamment son traitement politique et judiciaire en Belgique et au Rwanda (de Lame D., 2003, Vidal Cl. 2004). 
- Les répercussions au Congo et particulièrement au Kivu

Pour le Kivu proprement dit, la situation est restée si confuse jusqu'à l'époque actuelle que les spécialistes peinent à compter les conflits (Balencie J.P., 1999), (Kabamba B. et Lanotte O., 1999). On rappellera qu'au Nord-Kivu, plus spécialement dans le territoire de Masisi, une immigration rwandaise avait été organisée par l'administration coloniale dès la fin de l'entre-deux guerres pour procurer de la main-d'oeuvre aux plantations et élevages des colons belges. Elle s'était prolongée de façon spontanée par la suite et comportait une part importante de réfugiés Tutsi, surtout après 1960. Ces immigrés sont entrés en conflit pour les terres et les postes politiques avec les autochtones (généralement des Hunde) ce qui provoqua des affrontements violents et même des massacres. Au cours de l'année 1993, avant le déclenchement de la tragédie rwandaise, dans le territoire de Masisi, les populations autochtones hunde, avec l'aide de gendarmes congolais, massacrèrent des immigrés tutsi, provoquant ainsi le déplacement de plusieurs dizaines de milliers de personnes. Dans le territoire de Rutshuru, les Banyarwanda prirent les devants en massacrant leurs voisins hunde. Ces affrontements s'exacerbèrent encore suite à l'arrivée de réfugiés hutu fuyant le Rwanda en 1994. Ces conflits sont traités dans les articles de Tegera A. (1995), de Kafarhire Murhula (2003) et dans la première partie d'un ouvrage dirigé par Mathieu P. et Willame J.-C. (1999) (articles de Kabamba B. et Lanotte O., de Mathieu P., Laurent P.-J. et al., de Mathieu P. et Willame J.-C., de Mathieu P. et Mafikiri Tsongo, de Laurent P.J.). Ceux-ci décrivent une escalade conflictuelle autour d'enjeux fonciers et identitaires, la déstabilisation des paysanneries et certains évoquent ou proposent quelques remèdes possibles. Voir aussi pour le cas de Masisi, Vlassenroot K. (2004).

- Le problème de la nationalité

Parmi les facteurs intervenant dans ces conflits au Kivu, il y eut aussi la question de l'attribution ou du retrait de la nationalité zaïroise (congolaise) aux populations d'origine rwandaise vivant au Kivu. La politique du gouvernement zaïrois avait été très fluctuante sur cette question. (Kafarhire Murhula, 2003). Celle-ci avait abouti, dans le Sud-Kivu, au problème des Banyamulenge (Willame J.-C., 1997). On sait que la protection de ceux-ci a été un des prétextes invoqués par les nouvelles autorités rwandaises pour intervenir au Congo en septembre 1996. II y avait, au départ, les quelques milliers de pasteurs installés sur le haut plateau de l'Itombwe, dont parle G.Weis dans son étude sur le pays d'Uvira en 1959, arrivés dans le derniers tiers du XIXe siècle, puis différents immigrés tutsi venus par la suite, notamment au cours des remous de l'indépendance du Rwanda, dans la plaines de la Ruzizi. Le terme fut ainsi étendu à l'ensemble des Tutsi installés au Sud-Kivu et à qui le gouvernement refusait la nationalité zaïroise soit finalement plusieurs centaines de milliers de personnes. Celles-ci se heurtèrent aux groupes hutu réfugiés de 1994 ce qui, s'ajoutant aux incursions en territoire rwandais des milices hutu des camps de réfugiés du Nord-Kivu, déclencha l'intervention de la nouvelle armée rwandaise qui ramena une grande partie de ces réfugiés au Rwanda et conduisit d'autre part à la conquête du Congo au bénéfice de Laurent Kabila. Pour le Sud-Kivu, dans la région d'Uvira, voici une contribution importante à l'étude des affrontements entre d'une part les Barundi et les Bayamulenge et d'autre part les Bafuliira et les Bavira (Bosco Muchukiwa, 2006). Sur la question de la nationalité, on lira aussi l'article de Ndaywel è Nziem I. (2004) qui conclut qu'une solution à ce problème ne peut être trouvée dans le seul cadre congolais, ni même dans chaque pays séparément mais qu'elle doit résulter d'une concertation entre les différents pays concernés Des articles à caractère plus juridique ont été écrits sur le sujet. Nous ne les citerons pas ici.

Le numéro 84 de la revue française «Politique africaine» (décembre 2001) est consacré en grande partie à la guerre qui a sévi au Congo, sous le titre " $R D C$, la 
guerre vue d'en bas" et a l'ambition de faire connaître le point de vue des victimes. Le thème est Vivre dans la guerre. Imaginaires et pratiques populaires de la violence en RDC. Des dessins d'enfants, par exemple, illustrent les violences qu'a connues le Maniema. Sur le Kivu aussi, on retiendra la tentative de définition des Maï-Maï (Van Acker F. et Vlassenroot K., 2001): «une nébuleuse de milices et de bandes armées... dont la raison sociale combine dans des proportions variées la résistance à l'occupant rwandais, une logique d'épuration ethnique et le pur banditisme».

L'intégration aux Maï-Maï n'est-elle pas aussi une façon d'échapper à l'exclusion? Des jeunes marginalisés, exclus du monde scolaire, se révoltent contre l'environnement politique et social qu'ils rendent responsable de leur sort et s'expriment dans la violence. Ils ont trouvé un motif supplémentaire à leur action dans la résistance à l'occupant Tutsi. Pendant longtemps ces groupes armés ont été, dans les campagnes du Kivu, les seuls représentants d'une structure d'autorité. Ces milices issues du monde rural ne paraissent pas d'ailleurs s'être tournées contre celui-ci. Quel sera finalement leur impact sur l'ordre social local? (Vlassenroot. K., Van Acker F., 2001; Vlassenroot K., 2002).

Une équipe de chercheurs de l'Université de Kisangani a publié un intéressant recueil d'articles sur les événements qui se sont produits dans le Congo oriental de 1996 à 2007 (Maindo M.N.A., 2007).

Est-ce par suite du souvenir qu'avaient laissé les rébellions «mulélistes» des années 1960 dans la région, que malgré un certain repli identitaire et l'importance de sa population non autochtone (travaillant par exemple dans ses centres miniers) que le Maniema n'a pas connu d'importantes violences physiques, contrairement au Nord-Kivu? (N'sanda Buleli L., 2005).

\section{- Les réfugiés}

Les déplacements de réfugiés ont joué un rôle essentiel, comme conséquence et comme facteur, dans les guerres civiles. Un livre de Guichaoua A. (2004) traite de la question des réfugiés et des exilés pour l'ensemble de l'Afrique orientale. Les conclusions des articles publiés au moment des grands flux ont été rapidement dépassées. Un livre coordonné par un Père Blanc expose par exemple le problème des réfugiés rwandais (hutu) au Congo, à un moment où l'on craint que leurs camps ne deviennent pérennes comme ceux des réfugiés palestiniens dans le Proche-Orient (de Dorlodot Ph., 1995). On lira à leur sujet une mise au point d'un géographe allemand (Wiese B., 1997) et une bonne analyse du problème faite à chaud en 1997 par quelqu'un qui a participé à une mission des Nations-Unies (Mubiala M., 1997). Revenant quelques années plus tard sur cette question, R. Pourtier rappelle que les camps de réfugiés tutsi en Uganda puis de réfugiés hutu au Kivu ont été indissociables des conflits qui les ont fait naître, ont contribué à leur pérennisation et ont fonctionné comme des lieux de réorganisation politique et de bases de reconquête d'un pouvoir perdu. Pourtier rappelle aussi l'importance, dont on a moins parlé, des réfugiés de la nébuleuse kongo, résultant de flux dans les deux sens entre les deux Congos et surtout de flux de l'Angola vers le CongoKinshasa. Ceux-ci avaient commencé avec les révoltes angolaises de la fin de la période coloniale et se prolongèrent ensuite dans une interminable guerre civile. Ici les conditions ont été plus favorables à l'accueil et à l'intégration grâce à l'appartenance à la même entité. Au début 2002, le HCR dénombrait ainsi, dans le BasCongo, 81000 réfugiés non assistés contre 23400 assistés. (Pourtier R., 2006). Sur ces réfugiés du Bas-Congo, voir aussi une communication de Sabakinu Kivilu (2004). [En achevant la rédaction de cette chronique, nous apprenons que le sort de ces réfugiés est menacé par la dispute entre les deux Etats sur la délimitation de leurs zones d'exploitation océaniques respectives]. Sur les réfugiés dans l'est du Congo, on lira les communications de Bazenguissa R. (2004) et de Royer A. (2004) dans le livre dirigé par Guichaoua signalé plus haut. On rappellera aussi que des Hutu, surtout des miliciens et d'anciens militaires, fuyant le Kivu, sont arrivés 
au Congo-Brazzaville et ont été installés dans des camps dans la Likouala. Sur le séjour d'un groupe de réfugiés rwandais arrivé ainsi à Bamanya, près de Mbandaka, sur la rive gauche du Congo, en 1997, dans l'espoir de passer au Congo-Brazzaville, voici le témoignage d'un habitant de ce village (Bofeko Etaka, 2004). Malgré les différentes opérations militaires menées pour forcer les réfugiés à retourner au Rwanda tant au Congo que dans les pays limitrophes, comme en Tanzanie, certains réfugiés ont pu résister aux pressions exercées sur eux. A Nairobi, au Kenya, ils étaient encore entre 8000 et 15000 en janvier 1997. Un géographe a mené une enquête auprès d'eux en hésitant d'ailleurs à publier tous les résultats qu'il avait obtenus de peur qu'ils ne suscitent des réactions défavorables à leur égard (Cambrézy L., 1997 et 1998).

- Les interventions étrangères et les ressources minières

Dans une étude de Kennes E. (1997), outre une analyse des répercussions de la tragédie rwandaise sur le régime Mobutu incapable de les gérer au Kivu, on trouvera un exposé sur la façon dont des sociétés internationales ont tiré parti de situations troublées et indécises pour prendre en mains une partie du secteur minier, prise en mains déjà amorcée d'ailleurs par des négociations antérieures avec les gouvernements mobutistes. Au cours de la dernière décennie, on a souvent mis en relief le rôle que les ressources minières du Congo ont pu jouer dans les conflits: intervention de pays étrangers demandant en retour une participation à certaines exploitations, financement des rébellions par une partie de l'exploitation artisanale de l'or, du diamant, du coltan (minerai de colombo-tantalite). Le géographe R. Pourtier voit lui aussi, dans la «guerre continentale» qui a impliqué une dizaine de pays africains, une manifestation de pillage des ressources minières au profit des groupes dirigeants de ces pays et des réseaux financiers internationaux. La forme la plus aboutie de l'économie de prédation qui a toujours sévi sur le Congo depuis sa fondation? (Pourtier R., 2002).
Sur ce thème des pillages par les intervenants étrangers, voir aussi le livre d'historiens «radicaux» (Renton D. et al., 2007). Mayoyo défend la thèse d'un grand complot occidental, médias compris, contre le Congo (Mayoyo Bitumba Tipo-Tipo, 2006). Alors que l'exploitation artisanale des produits miniers était considérée, il y a peu encore, comme la forme la plus accomplie des systèmes de débrouille, elle est maintenant criminalisée dans la mesure où le contrôle et l'exportation de la production sont entre les mains d'étrangers (rwandais et autres) accusés de piller le pays (Jackson S., 2001).

\section{Les dernières années}

Dans la foulée des réflexions qui précèdent, voici une lecture géopolitique de I'histoire congolaise depuis l'indépendance et surtout depuis 1990. Elle analyse le rôle des grandes puissances et particulièrement celui de la «troïka» occidentale (Etats-Unis, France, Belgique) dans la transition zaïro-congolaise. Elle s'interroge sur une logique «statocidaire», c'est-à-dire visant à la destruction d'un Etat, dans ce cas-ci pour s'emparer de ses richesses, logique qui aurait été celle de ses voisins proches (Rwanda, Uganda) ou lointains (comme le Zimbabwe et l'Afrique du Sud) (Ntumba Luaba Lumu, 1999).

Un article critique cependant un certain nombre d'idées émises sur la situation congolaise en examinant l'ère Mobutu (jusqu'à 1997) et la guerre (1996-2003) et en tirant quelques conclusions de la longue période de «transition» politique. C'est ainsi que la RDC est présentée souvent comme un exemple de faillite et d'effondrement d'un Etat. Pourtant elle montre une remarquable capacité de «résilience», comme on dit aujourd'hui, une étonnante capacité à rebondir. Quoi qu'on puisse dire sur leur fonctionnement réel, les structures administratives par exemple se sont maintenues. La période de guerre a été une continuation de la violence par d'autres moyens. Des réseaux politiques mafieux ont été remplacés par des réseaux militaires (rebelles, milices, «entrepreneurs de l'insécurité»). Le rapport de l'ONU, qui décrivait une organisation criminelle visant 
à dépouiller le Congo de ses ressources, ne serait qu'une vue très tronquée de la réalité. Des organisations politiques nouvelles ont joué, au moins temporairement, un rôle non négligeable, comme le Rassemblement Congolais pour la Démocratie, RCD-Goma. L'évolution de I'Etat correspond-elle à une privatisation ou à une banalisation de sa souveraineté (Vlassenroot K. et Raeymaekers T., 2008)? En octobre 2006, la nouvelle République démocratique du Congo a procédé à ses premières élections avec l'aide de la communauté internationale. Pour les préparer, la population de plus de 18 ans a dû se faire enrôler en 2005 pour obtenir une carte d'électeur. Le taux d'enrôlement (pourcentage des enrôlés par rapport à la population attendue calculée à partir des données du recensement de 1984) a été de l'ordre de $90 \%$. Les deux tiers des enrôlés ont participé au référendum sur la nouvelle Constitution en décembre 2005 qui a été approuvée par $84 \%$ des votes valables. Les cartes et un tableau donnent ces valeurs par territoire, y compris la population totale attendue qui était de 59 millions pour l'ensemble du Congo, étrangers non compris (de Saint Moulin L., 2006 a). Cet article figure dans un numéro de CongoAfrique consacré au compte rendu des
Journées sociales du Centre d'études pour l'action sociale (Kinshasa) sur le thème «Elections et bonne gouvernance pour la Ille République en RDC: avec quels dirigeants et quel projet de société?»

L. de Saint Moulin a dressé ensuite les cartes des élections présidentielles. Au premier tour au scrutin du 30 juillet 2006, Joseph Kabila domine dans la partie orientale du pays, Jean-Pierre Bemba, dans le nord-ouest et une partie du Kasai, Antoine Gizenga dans la province de Bandundu (de Saint Moulin L., 2006b). Au second tour, le candidat Bemba étend son emprise sur le Bas-Congo, le nord du Bandundu et une grande partie des deux Kasaï. Le vainqueur, Kabila, a renforcé sa position dans la partie orientale du pays et notamment au Katanga, avec des majorités beaucoup plus élevées que celles obtenues par son concurrent dans les circonscriptions où ce dernier l'emporte. Les deux articles comportent un tableau des résultats par territoire ou ville (de Saint Moulin L., 2007). On trouvera aussi les cartes du deuxième tour, leur commentaire ainsi qu'un aperçu du processus électoral dans BELGEO, qui sur son site (www.srbg.be) a présenté ces cartes en couleurs, avec les tableaux des données (de Saint Moulin L. et Wolff E., 2006).

\section{LA VIE ÉCONOMIQUE ET SOCIALE DES CAMPAGNES}

LES GROUPES ETHNIQUES TRADITIONNELS, LEURS TECHNIQUES D'EXPLOITATION DE LA NATURE ET LEURS TECHNIQUES D'ENCADREMENT

\section{Généralités}

On trouvera des rubriques généralement bien au point sur différents groupes ethniques congolais dans le tome consacré à I'Afrique et au Moyen Orient d'une grande encyclopédie sur les cultures mondiales (Middleton J. \& Rassam A., 1995). Quelque peu curieux est, dans une autre collection américaine, l'ouvrage qu'un professeur africain de l'Université d'Atlanta (Géorgie) consacre aux cultures et aux coutumes du Congo. Ce qui est dit, par exemple sur l'art traditionnel ou sur la mai- son villageoise, est d'une brièveté déconcertante. II faut dire que les rubriques sont nombreuses: religion, littérature, art, architecture, cuisine, habillement, coutumes sociales et style de vie, mariage, famille, "gender roles», musique et danse (Tshilemalema Mukenge, 2002).

\section{Les ethnies et l'ethnicité}

Les affrontements entre groupes ont donné un sens tragique à la notion d'ethnie. Plusieurs colloques internationaux ont été consacrés à cette question. Dans le but de contribuer à une meilleure connaissance du pays par ses habitants et dans l'espoir ainsi de leur permettre de développer une culture de la paix, L. de Saint Moulin a dressé des cartes ethniques régionales du 
Congo. II reconnaît d'abord que la notion d'ethnie est très floue. Si l'ethnie est considérée généralement comme une unité supérieure à la tribu, les deux termes sont utilisés souvent l'un pour l'autre. Les dénominations sont de provenance variable et souvent ont été données par des voisins. Ainsi, pour L. de Saint Moulin, l'ethnie est une construction établie sur des bases diverses mais non systématiques. «Les identités ethniques..., écrit-il, sont des stratégies sociales. Mais elles ne sont pas une simple traduction du présent. Elles sont des constructions historiques». Ses cartes veulent renouveler celles qui accompagnaient l'Introduction à l'ethnographie du Congo, de Jan Vansina (1966). Celui-ci cherchait à représenter la situation avant l'occupation européenne mais il reconnut par la suite que cet objectif était fallacieux. La situation représentée était en fait celle de la fin de la période coloniale. Les cartes de L. de Saint Moulin sont réparties en huit grands ensembles au 1/5 000 000. Deux annexes fort utiles donnent la liste des ethnies et tribus par territoire, soit 281 au total et celle des collectivités (759), subdivisions administratives des territoires (de Saint Moulin L., 1998). L'auteur revient sur le sujet en 2002, en rappelant comment la notion d'ethnie s'est modifiée pendant la période coloniale.

Dans la trame administrative coloniale, comment s'est faite la prise en considération des ethnies, comment celles-ci ontelles été identifiées et se sont-elles affirmées, de quelle façon leur rôle s'est-il maintenu depuis l'indépendance? $P$. Salmon traite ces questions dans le cas de l'ethnie zande, unité culturelle composite (Salmon P., 1998).

Voici une pièce à verser au débat sur l'origine et la signification des ethnies actuelles et sur celles de leur nom. Les Topoke du territoire d'Isangi, en aval de Kisangani, dans la Province Orientale (Haut-Zaïre), sur l'Equateur, sont connus sous huit noms plus ou moins différents. Le mot Topoke lui-même signifierait simplement «nous ne comprenons pas», première réponse sans doute aux questions des agents coloniaux. Leur vraie dénomination est-elle encore inconnue? (Bilusa Baila Boingaoli, 1993).
Sur la difficulté à définir une ethnie et en tout cas à accepter un ethnonyme, L. de Heusch rappelle le cas des Tetela. Cet ethnonyme est un produit de l'histoire coloniale. II s'agit en fait de Nkutshu. Le terme tetela est apparu au moment où les troupes de Ngongo Lutete ont enrôlé (de force) les hommes des villages nkutshu qu'ils conquéraient (de Heusch L., 1995). Le politologue américain Thomas Turner a étudié longuement les Tetela. II décrit, dans un livre écrit directement en français, tous les éléments historiques, généalogiques réels ou imaginaires et les interventions extérieures qui ont construit cette ethnie. On ne peut comprendre la trajectoire de Patrice Lumumba si l'on ne tient pas compte de ce qu'il appartenait à l'ethnie tetela mais que cette appartenance n'a pas empêché (et au contraire a peut-être favorisé) sa vision transethnique de la politique congolaise (Turner T., 2000).

Comment être un Luba au XXe siècle? Le livre qui pose cette question traite en fait uniquement des Luba du Kasai, ce groupe qui a connu une forte expansion pendant la période coloniale, qui a fourni aux centres miniers du Katanga une grande partie de leur main-d'oeuvre et, qui, avec un certain soutien des autorités publiques et de l'Eglise catholique, a occupé beaucoup de postes subalternes dans l'administration. Ces Luba du Kasai ont été expulsés par la suite en 1960 des terres lulua, près de Luluabourg, et surtout du Katanga minier (une nouvelle fois en 1992). Ainsi cette identité Luba du Kasai «relève moins d'un univers de traditions ancestrales que d'une recomposition sociale et culturelle de l'époque coloniale». Ils ont joué du christianisme comme d'un nouveau cadre identitaire (Kalulami Pongo M., 1997). Très curieusement ce livre ne parle pas des Luba du Katanga ni de leurs liens avec les Luba du Kasai ni des grandes constructions politiques luba précoloniales. II ignore en outre totalement les recherches menées par P. Petit, sur l'ethnie luba.

Or Pierre Petit, qui défendra, en 2003, à I'ULB, une thèse sur les rites familiaux et royaux des Luba, avait écrit, en 1996, un article très riche sur l'ethnicité luba. L'ethnonyme est attesté avant l'occupa- 
tion coloniale d'abord sous sa forme swahilisée, Warua. L'ethnie luba «doit... se concevoir non pas comme un ensemble discret mais comme un ensemble polarisé, auquel un individu peut s'identifier ou non, selon les contextes». A son extension maximum (vers 1800), le royaume luba était composé de cercles concentriques avec décroissance progressive du pouvoir royal depuis la capitale. Le terme luba a parfois été utilisé pour désigner uniquement le roi (par l'explorateur Cameron, par exemple), parfois aussi les dignitaires de la cour royale et tout ce qui se trouve au centre du royaume. Chaque souverain construisait sa capitale, d'abord un campement de chasseurs, le musumba, puis le dipata, ensemble constitué par le palais entouré des maisons des dignitaires. La construction du dipata était accompagnée de sacrifices humains (généralement d'un "pygmée» autochtone), les victimes devenant ainsi les premiers occupants de la terre et les protecteurs des hommes vivant sur ces lieux. A la mort du roi, la capitale était progressivement abandonnée mais son site conservait ses caractères sacrés. Le pays est ainsi constellé de sites historiques (Petit P., 1996 a). Sur ces capitales disparues mais dont les sites sont encore visibles, parmi les royaumes voisins, notamment le royaume kanyok, voir l'article de R.Ceyssens, commenté dans le paragraphe sur les villes précoloniales. (Ceyssens R., 2007). Le roi luba est le prolongement vivant de ses prédécesseurs comme le matérialisent trois institutions: le culte des ancêtres royaux, l'épreuve d'intronisation qui marque l'accord des esprits au candidat-roi, l'emploi de reliques pour transmettre un principe spirituel (Petit P., 1996 b).

C'est pour le Rwanda, avant et après la tragédie de 1994, que la notion d'ethnie a suscité le plus de commentaires. Danielle de Lame, avant 1994, avait rappelé que l'hypothèse hamite (avec les Ethiopiens, les Tutsi auraient été apparentés à des Caucasiens comme les Berbères) avait gardé ses partisans jusqu'en 1960. D'autre part, toutes les informations recueillies par les missionnaires mais aussi par les anthropologues l'avaient été auprès d'informateurs Tutsi (de Lame D., 1993).

Pendant l'année qui a vu la fin du pouvoir tutsi et le triomphe électoral des Hutu, comment les trois groupes (Tutsi, Hutu et Twa) se percevaient-ils? Une enquête faite sur 252 Rwandais, à l'époque, avait montré que les réponses des personnes interrogées reflétaient les préjugés de chaque caste vis-à-vis des deux autres tout en concédant que les relations politiques entre les castes avaient changé (Codere H., 1993).

Tout Rwandais était intégré à l'un des 18 clans qui mêlaient Tutsi, Hutu et Twa. A partir d'un échantillon de plusieurs dizaines de milliers d'individus, en 1960, d'Hertefelt a fait l'hypothèse que certains clans ont été à l'origine de groupements territoriaux, autour d'un bloc central plus densément peuplé (Vidal Cl., 1993).

On trouvera d'autres commentaires sur les aspects ethniques des événements du Rwanda, dans les ouvrages et articles que nous avons cités dans la partie historique de cette chronique. Voir notamment de Saint Moulin L., 2002; Chrétien J.-P., 1997; Chrétien J.-P. et Mururi M., 2002; Chrétien J.-P. et Prunier G., 2003; Reyntjens F., 1993; Vansina J., 1993 et 2001; Williame J.-C., 1997.

Au Burundi, on a vu récemment un groupe revendiquer une identité ethnique. I s'agit des Bangwana, c'est-à-dire des princes tutsi de sang royal qui formaient un groupe politique dans l'ancien royaume du Burundi et se prétendaient une catégorie sociale distincte des Hutu, des Tutsi et des Twa. Favorisés parfois par l'administration coloniale pour les postes de chefs coutumiers, ils ont perdu toute importance après l'avènement de la République. Depuis les réformes constitutionnelles de 1992, ils cherchent à retrouver un rôle politique et veulent être reconnus comme une ethnie à part entière. Leur résurgence a été une des manifestations de la crise de la citoyenneté burundaise et est une expression supplémentaire du clivage ethnique (Mworoha E. et Chrétien J.-P., 2003).

Les tensions qualifiées d'ethniques au Sud-Kivu, particulièrement dans le territoire d'Uvira, entre Barundi et Banyamu- 
lenge d'une part, Bafuliiru et Bavira, de l'autre, sont surtout des conflits fonciers, résultats d'une dynamique territoriale d'appropriation de la terre par des immigrés récents (Bosco Muchukiwa, 2006). Un ouvrage traite des mêmes problèmes au Maniema (N'Sanda Buleli L., 2005).

Les institutions traditionnelles et leur transcription dans la société moderne

Chez les Mbo, groupe de cultivateurs et de pêcheurs, sur les rives de l'Epulu, dans I'Ituri, l'institution du Nkumi, rite de circoncision, est un puissant facteur d'intégration des groupes en présence, face aux pygmées mbuti. Cette institution forme la base d'une confédération politique qui transcende les différences linguistiques et tribales et a même été acceptée par des groupes musulmans (Towles J.A., 1993).

Les Luntu, qui habitent la province du Kasai oriental, près de Dimbelenge, ont une association du léopard, comme les Mongo, qui remplit d'importantes fonctions politiques et religieuses (Petridis C., 2000). Chez les Songye (Kasai oriental), le titulaire d'une charge politique cède sa place, après quelques années, à un membre d'un autre groupe de parenté de sorte que chaque groupe accède, à son tour, au pouvoir (Anthoine C., 1996).

II paraît indéniable que Chokwe et Lunda (aLuund) sont deux groupes distincts, parfois antagonistes dès avant la période coloniale. Pourtant, dans le sud du Kwango, les Lunda n'hésitent pas à recourir aux Chokwe pour des interventions thérapeutiques, en faisant appel à leurs devins, guérisseurs et autres spécialistes rituels (De Boeck F., 1993).

II faut citer, dans ce domaine des rites thérapeutiques, l'article du regretté Stefan Bekaert sur les Sakata (rive droite du Kasai, province de Bandundu) (Bekaert S., 1996).

Dans la même province, au sud du Kasai, un article décrit les structures de parenté des Yansi du territoire de Bagata. II montre comment les stratégies matrimoniales assurent la circulation des femmes et des titres fonciers. L'appropriation de la terre concerne l'affectation à un usage et dispa- raît avec cet usage, par exemple lorsque la jachère n'est plus cultivée; défricher un champ garantit ainsi des titres fonciers à sa descendance. La législation de l'Etat moderne ne tient pas compte du système traditionnel et est donc une source de conflits (Mfukula Moke Key W.-R., 1994).

Revenant en pays lele, une trentaine d'années après les recherches qu'elle y avait menées, Mary Douglas constate qu'un mouvement anti-sorciers, dirigé par deux prêtres catholiques à partir de la fin des années septante, a eu pour résultat de faire ranger tout ce qui était du domaine des rites traditionnels dans le domaine des démons et du diable, ce qui donne ainsi, au sein du christianisme, une place aux croyances locales dans la sorcellerie (Douglas M., 1999).

Pendant l'époque coloniale, à l'exception de L. de Heusch et de J. Maquet, on a très peu comparé les institutions du Rwanda avec celles des autres civilisations interlacustres. Les spécialistes du Rwanda par exemple se seraient même peu préoccupés de comparer les institutions du Rwanda avec celles du Burundi (Trouwborst A., 1993).

Une institution cherche à refaire surface au Burundi, celle des bushingantahe qui étaient chargés autrefois d'arbitrer les conflits sur les collines avec aussi une mission de conseil auprès de la population. Depuis les réformes de 1992, ils cherchent à intervenir dans les élections communales. Un conseil de 40 bushingantahe a été créé pour émettre des avis sur les parlementaires nommés pour représenter la société civile. Mais l'avenir de cette institution ne paraît pas assuré (Deslaurier C., 2003).

Sur les relations entre le pouvoir "moderne» et les chefs détenteurs du pouvoir traditionnel, et notamment sur les malentendus ou les ambiguïtés du rôle que s'attribuent les deux parties, on lira le cas des relations du chef lunda de Kahemba avec le pouvoir mobutiste dans un article de F. De Boeck (1998 a). Les chefs traditionnels interprètent les actes du pouvoir, qui cherche à se les attacher, comme une reconnaissance de leur suzeraineté. Le poids du chef luunda du sud du Kwango par rapport à celui du Katanga s'est ren- 
forcé depuis l'exploitation artisanale du diamant et le trafic du diamant angolais.

Les techniques traditionnelles d'exploitation de la nature

Une analyse originale des fronts pionniers, qui attaquent la grande forêt de I'Ituri depuis un siècle, s'inspire de concepts écologico-culturels et de critères utilisés pour des études du même type en Amazonie. Elle envisage la concurrence entre les groupes, leur perception de l'espace comme ressource et la façon dont se structurent les relations de pouvoir. L'Etat colonial ou postcolonial n'est pas intervenu institutionnellement dans cette colonisation des terres (Rösler M., 2004).

Voici une description des techniques et de leur évolution pour des riverains de la Ngiri et de l'Ubangi, en forêt équatoriale: aménagement de l'habitat (hameaux sur des tertres artificiels, villages-îlots), techniques de navigation, de pêche, d'agriculture, techniques artisanales (vannerie, tissage, métallurgie, poterie -apanage des femmes-, extraction de l'huile, du vin de palme, du sel végétal) (Mumbanza mwa Bawele, 1995).

Pour d'autres peuples de la grande forêt, étudiés tout particulièrement près de Mbandaka, dans le bassin de la Ruki, voici un article sur les Elinga, c'est-à-dire des gens d'eau disséminés dans l'aire mongo (Kanimba Misago, 1995).

Les pêcheurs Ntomba, autour du lac Tumba, dans la forêt inondée, exercent leur activité dans un contexte magico-religieux. Ils dépendent du bon vouloir des génies bilima qui dispensent les poissons pour les femmes, le gibier pour les hommes. Les campements de pêche et la pêche collective sont régis par des maîtres de pêche, descendants du premier occupant qui avait conclu un pacte avec le génie, chef du lieu (Pagezy $\mathrm{H}$., 2006). Sur les problèmes alimentaires des Ntomba, voir plus loin.

Sur l'avenir des peuples des forêts tropicales, une vaste étude a été menée dans le cadre d'un programme financé par la Commission Européenne. Elle a fait l'objet de gros rapports et d'un CD-rom de pho- tos en couleurs, où les relations entre ces peuples et leur milieu sont étudiées de façon approfondie (Bahuchet $S$. et de Maret P., 2001). Le premier des quatre rapports est une présentation générale de la question que l'on retrouve succinctement dans un article qui rappelle, dans son titre (Forêts des tropiques, forêts anthropiques), que la grande forêt équatoriale est un milieu profondément transformé par l'homme (de Maret P. et al., 2002). Le volume III concerne l'Afrique centrale. Son chapitre 5 traite de la RDC Le gel de la coopération européenne n'a pas permis d'y mener des recherches aussi approfondies que dans d'autres pays. Les gouvernements Kabila ne sont pas apparus non plus très soucieux de la protection de l'environnement et ont envisagé pour soutenir leurs actions, notamment leurs actions militaires, d'intensifier l'exploitation forestière. L'étude couvre quatre sites de recherches principaux: Kinshasa, Kikwit, le Kivu, le parc national de la Garamba. A Kinshasa, ont été spécialement abordées la déforestation liée aux besoins alimentaires, la ruralisation de certains quartiers de la ville, la pénurie du bois de chauffe et du charbon de bois, la nécessité d'un plan stratégique global impliquant les acteurs locaux de tous niveaux et la communauté internationale. A Kikwit, c'est aussi le thème de la déforestation de I'hinterland de la ville qui a été traité. Le Parc National de la Garamba, menacé par le déclin de sa population de grands mammifères, a été pris comme exemple d'un transfert de responsabilité aux institutions locales qui auraient à réglementer elles-mêmes les captures permises aux chasseurs locaux. Malgré une situation particulièrement troublée et violente, des enquêtes ont pu être menées parmi les populations riveraines des deux Parcs Nationaux du Kivu (Virunga et Kahuzi-Biega) notamment sur leur perception du rôle et de l'importance de leurs écosystèmes forestiers. Parmi les questions qui se sont posées, il y a celle du maintien de groupes pygmées dans le Parc National de Kahuzi-Biega. Les Pygmées sont le groupe qui a été le plus marginalisé par la création des Parcs Nationaux. L'étude envisage aussi les 
effets écologiques des événements qui ont bouleversé le pays (guerres civiles, opérations militaires, mouvements de réfugiés, etc.) (de Maret $P$. et al., 2000). Le type et la liste des questions qui ont été posées au cours des enquêtes sont exposés succinctement dans un article qui explique la nature et le but du projet qui a été poursuivi (de Maret et al., 2004).

Les Hema du Sud, peuple pasteur du nord-est du Congo (Haut Ituri), au-dessus du lac Albert, autour de Bunia, sont décrits dans une vaste étude ethnohistorique, genre particulièrement pratiqué par des missionnaires, à partir des traditions orales (l'auteur est un Père Blanc d'Afrique) mais aussi des sources écrites de l'époque coloniale. Cette étude contient une grande masse de données mais n'apparaît pas toujours d'une très grande clarté. Elle fournit quelques indications sur les relations entre les Hema et les groupes paysans auprès desquels ou au milieu desquels ils vivent mais ne fait que quelques allusions aux tensions et au risque d'affrontements entre groupes ethniques tels qu'ils se sont produits au cours de la dernière décennie. Les Hema sont apparentés aux Hima de la région interlacustre. Les Hema du Sud ont conservé l'usage de la langue nyoro (ils seraient originaires du Bunyoro en Uganda) tandis que les Hema du Nord ont adopté la langue des groupes lendu parmi lesquels ils sont implantés. L'activité pastorale joue un rôle plus important chez les Hema du Sud que chez les Hema du Nord (Thiry E., 1996). On signalera une bibliographie des Shi du Sud-Kivu (autour de Bukavu) (Mwenze D., 1999).

Sur la persistance d'activités traditionnelles, on retiendra le mémoire que Pierre Petit consacre aux sauniers du Katanga et des régions voisines de la Zambie, c'est-à-dire dans une aire qui se trouve à l'intérieur des royaumes luba, lunda et bemba et de l'espace linguistique lubabemba. L'extraction du sel, qui se pratique en saison sèche, assure une production annuelle de 20 à 40 tonnes par saline soit 1000 à 1500 tonnes pour l'ensemble de la région étudiée. Cette acti- vité s'est maintenue à peu près telle qu'elle était à la fin du XIXe siècle. La crise économique actuelle, qui impliquerait un repli sur des activités traditionnelles, n'explique qu'en partie sa persistance. Celleci correspond surtout au fait que le saunage représente un fait social total qui intègre les sphères de la vie économique, politique et symbolico-religieuse et est perçu comme un élément de patrimoine. Les populations du Katanga gardent leur préférence à un sel traditionnel et les chefs locaux mettent toute leur énergie à défendre leurs droits sur les salines. Le mémoire décrit avec finesse les processus techniques, les réseaux sociaux autour de cette activité, notamment par l'utilisation monétaire du sel, les circuits commerciaux et le cadre religieux qui, comme dans le cas cité plus haut des capitales des rois luba, comporte un culte aux esprits territoriaux rendu possible, au départ, par une cérémonie sacrificielle, qui pouvait être jadis la mise à mort de l'inventeur des lieux (Petit P., 2000).

Les paysages agraires, l'habitat et l'habitation

On trouvera quelques exemples de structures des paysages agraires d'Afrique centrale dans un article de réflexions sur les caractères originaux des paysages agraires de l'Afrique tropicale (Nicolaï $\mathrm{H}$., 1996).

Sur l'habitat dispersé des hautes terres orientales, on citera plus loin des articles sur ses problèmes et sur de nouvelles opérations de regroupement.

Sur le thème de la maison «traditionnelle», un des thèmes privilégiés des géographes de la première moitié du dernier siècle en Europe et des ethnologues en Afrique, presque plus rien n'a été publié après 1965. Un article sur la maison cheffale des Pende est donc particulièrement bienvenu. La recherche a été menée en 1987 et 1988. Alors que beaucoup de Pende, du Kwilu comme du Kasai, y compris les chefs, construisent de plus en plus de maisons «en dur» (en briques adobe, par exemple), la case "officielle» du chef, sa résidence, le kibulu, ne peut être construite en matériaux durables. Le 
kibulu est une case carrée de $3 \mathrm{~m}$ de côté, faite de matériaux végétaux avec un toit de paille en coupole dont l'extrémité supérieure est ornée d'une sculpture et avec un vestibule précédant la porte. Cette case dure rarement plus de douze ans et doit donc être reconstruite périodiquement. A la mort du chef, le kibulu est abandonné. La construction du kibulu du nouveau chef obéit à un rituel précis. Des semences de millet sont enfouies dans le trou (le «ventre»), où sera planté le poteau central et qui deviendra ainsi le "coeur du village» et un symbole de fertilité. Le kibulu est donc un espace intensément religieux. On notera que le chef ne peut pénétrer dans la deuxième chambre du kibulu. Seul un ministre en a le droit ce qui signifierait symboliquement que le chef n'a pas tous les pouvoirs. Le caractère non durable de la case cheffale, son «impermanence», serait une autre expression de cette limitation du pouvoir. Des têtes sculptées au sommet des piquets de la palissade, devant l'entrée, surveillent d'ailleurs l'intérieur, donc le chef. La reconstruction périodique de la case cheffale exprime aussi la renégociation du contrat social entre le chef et son peuple (Strother Z., 2004). Signalons que la même auteure a publié d'importants ouvrages sur l'art des Pende et tout particulièrement sur leurs masques (Strother Z., 1998, 2005, 2008). Pour elle, les danses masquées ont été, pendant la période coloniale, des manifestations de l'identité pende et une forme de résistance au colonisateur. Nombre de nouveaux personnages ont été introduits dans l'univers des masques. Cette période a connu ainsi une grande création artistique, ce qui ne sera plus le cas après l'accession du Congo à l'indépendance.

On trouvera dans un article de De Boeck F. (1995), sur lequel nous reviendrons plus loin, des informations sur l'organisation de l'espace dans l'habitation des Lunda du sud du Kwango et plus spécialement sur l'opposition entre le devant et le derrière. Devant la maison, l'espace est surtout masculin, délimité et réaffirmé chaque matin par le chef de famille, lors du balayage de la cour. C'est là qu'il accueille les visiteurs. L'arrière est un domaine féminin. Les enfants s'y attroupent et les femmes s'y asseyent en préparant les repas. On y emmagasine le bois pour le feu, on y sèche le manioc et les arachides, on y trouve le mortier, la passoire, la houe, etc.

\section{Les systèmes fonciers}

Dans le cadre d'un grand programme de l'administration coloniale pour une prise en compte des structures foncières dans l'application des grands projets de développement rural, une enquête avait été menée en 1960, dans un groupe tetela du territoire de Lubefu (actuel Kasai oriental). Restée inédite, elle vient d'être publiée (Derolez C., 2006).

En pays nande, dans la localité de Luhotu (Nord-Kivu), une analyse multivariée indique qu'il n'y a pas de relation entre le système foncier et le système d'exploitation du sol. Une loi foncière de la fin du régime mobutiste, qui aurait dû protéger les petits paysans, a été utilisée pour permettre l'accaparement des terres par des fonctionnaires et a accéléré l'individualisation des droits fonciers (Mafikiri Tsongo A., 1994). La question est reprise par le même auteur pour montrer comment se combinent les règles coutumières et les lois foncières nationales. Cette combinaison entretient un sentiment d'incertitude donc d'insécurité pour les paysans traditionnels. L'article analyse tout particulièrement les problèmes que posent les acquisitions foncières des immigrés rwandais dans la zone de Masisi où les autochtones (Hunde) estiment avoir perdu ainsi une grande partie de leurs terres. II en est résulté des affrontements sanglants dans les années qui ont précédé la rédaction de cet article, affrontements qui prendront une ampleur accrue dans les années suivantes (Mafikiri Tsongo, 1996).

Les questions foncières sont traitées de façon plus générale dans un article qui montre qu'à côté du droit traditionnel et du droit formel moderne, apparaît un troisième système juridique. Par exemple, dans la province de Bandundu, on a autorisé l'accaparement individuel par les exploitants eux-mêmes de terres exploitées sur un mode traditionnel alors 
que ces terres relevaient de la communauté. Des agriculteurs, qui se désignent comme de "petits fermiers», ont réussi, avec l'accord du chef de terre traditionnel et grâce à quelques papiers obtenus des fonctionnaires locaux, à développer un nouveau droit foncier informel, ce qui crée une grande insécurité juridique. Au fond l'Etat a reconnu ainsi indirectement l'autorité des chefs de terre traditionnels supprimée théoriquement par la loi foncière de 1973 (Beke D., 1996).

LES PROBLÈMES DES HAUTES TERRES ORIENTALES (RWANDA, BURUNDI, KIVU, ITURI)

Les problèmes des régions du Congo oriental, du Rwanda et du Burundi sont replacés dans le cadre général de l'Afrique des Grands Lacs dans un article qui analyse les ruptures et les recompositions engendrées par les guerres dans la production agricole (les baisses de production ont été en partie compensées par l'aide alimentaire internationale), les déplacements temporaires ou définitifs, les relocalisations autour de centres administratifs. II prend notamment comme exemple les conséquences des regroupements forcés de paysans hutu, organisés par une armée à dominante tutsi au Burundi, qui ont déstructuré des campagnes entières et entraîné des situations de disette. II ne croit pas que les solutions aux problèmes de ces territoires puissent être envisagées en dehors d'un modèle à prépondérance rurale, ni sans l'arrêt de l'emballement démographique (Cazenave-Piarrot A., 2007).

Dans l'Ituri, des densités de population supérieures à 300 habitants au $\mathrm{km}^{2}$ se rencontrent au pied des Monts Bleus au dessus du lac Albert. Ici aussi on trouve les mêmes problèmes d'érosion des sols, de déboisement ainsi que les conflits fonciers entre agriculteurs proprement dits et éleveurs de bétail. Les auteurs de l'article pensent qu'une redistribution de la population pourrait aplanir beaucoup de difficultés. Mais, dans les conditions actuelles du pays, cette option est-elle réaliste et qui pourrait se charger de la promouvoir et de l'effectuer? (Nyori I. A. et Buga B.L., 1996).

\section{Rwanda}

Plusieurs grandes études portant sur les paysanneries des hautes terres ont été accomplies ou publiées au début de la dernière décennie du XXe siècle.

Un économiste, à partir de l'étude des pratiques d'élevage, a analysé les transformations de la colline de Gafunzo, dans la préfecture de Gitarama, au Rwanda. Une grande partie de la population se trouve dans une situation d'auto-subsistance. La croissance démographique et la raréfaction des terres disponibles la contraignent à une intensification des techniques. Dans le domaine de l'élevage, cela s'est marqué par le recours à la stabulation permanente du bétail. Une minorité de la population a accès à des emplois salariés (auprès de l'administration ou d'organismes de développement) ce qui, malgré une certaine redistribution de ses revenus, qui se fait souvent de caractère ostentatoire ou symbolique, n'empêche pas l'apparition de tensions avec les autres groupes. La création d'une entreprise hydraulique à laquelle n'a accès qu'une partie de la population est un autre facteur de disparité. L'étude analyse l'articulation de ces facteurs avec la dynamique propre de la colline (Migeotte F., 1997).

Un important ouvrage d'anthropologie rurale qui reprend l'essentiel d'une thèse soutenue, en 1996, à la Vrije Universiteit Amsterdam, décrit la vie et les problèmes d'une «colline» (c'est-à-dire d'une communauté rurale) du Rwanda, la colline de Murundi et son annexe de Gizebeya, qui forment ensemble la zone de développement rural de Munzanga, dans le centreouest du Rwanda (on regrettera, au passage, qu'aucune carte ne permette de la situer avec précision). Ce petit espace rural compte 180 enclos (ou familles nucléaires). L'auteure, Danielle de Lame, rappelle d'abord les caractéristiques générales des sociétés rurales du Rwanda dans une perspective historique. Elle décrit les liens sociaux, le vécu quotidien, les systèmes de relations. Elle analyse ensuite les échanges économico-symboliques fondamentaux qui font circuler les boissons, les vaches et les femmes. La 
lecture de cette étude anthropologique holiste d'une communauté rurale est indispensable à qui veut essayer de comprendre, de l'intérieur, le milieu rural rwandais (de Lame D., 1996). Une postface expose la vision de l'auteure sur les événements dramatiques de 1994. Elle y rappelle cette phrase atroce d'un de ses informateurs en 1990: "Quand on a semé trop serré, il faut sarcler». Bien qu'elle affirme avoir été, au cours de son enquête, pessimiste sur l'évolution du pays, il n'en reste pas moins que le titre même de l'ouvrage (le calme avant la tempête) témoigne que l'on croyait encore avant 1990 que le calme exprimait au moins une volonté ou un souhait de cohésion et qu'il n'annonçait pas de tempête.

Dans une autre publication, la même auteure montre comment toute étude en milieu rural est indissociable d'une anthropologie du changement socio-culturel (de Lame D., 1997). Or les enquêtes en milieu rural sont affectées de nombreux biais. II en est ainsi dans la détermination des priorités pour lesquelles l'opinion du paysan est sollicitée. En fait, celui-ci n'a généralement le choix que parmi les alternatives que le chercheur ou le développeur lui présente et qu'il croit les plus pertinentes. Les réponses sont ainsi en partie suggérées aux paysans interrogés.

Un autre ouvrage fondamental, issu lui aussi d'une thèse doctorale, cette fois de géographie, a été publié sur le Rwanda. Après avoir décrit les traditions rurales, F. Bart montre comment le paysan rwandais a relevé le défi démographique et a réagi aux crises qui le frappaient: accroissement de la superficie cultivée vers les sommets, mise en valeur des marais, colonisation des régions orientales plus basses et moins peuplées, intensification de l'exploitation y compris des techniques d'élevage (bovins à l'étable, chèvres au piquet), recherche de revenus monétaires, soit issus de l'agriculture (bière de banane, café) soit provenant de quelques gains salariés, par exemple au service d'organismes de développement et d'ONG diverses. Mais le pays s'est trouvé menacé par des situations de blocage dont les solutions ne pourront être trouvées dans les campagnes ellesmêmes, où les terres sont proches de la saturation. On s'étonnera qu'aucune référence ne soit faite au problème politique, c'est-à-dire aux tensions entre Hutu et Tutsi (c'est à peine si les mots sont utilisés dans le texte), sous prétexte qu'elles n'auraient eu aucun effet géographique visible et échapperaient ainsi au domaine traité par le géographe. Les événements qui ont suivi ont démenti cette vue des choses. Ou bien s'agissait-il de points auxquels le chercheur ne pouvait toucher sans danger pour ses possibilités de travail ? (Bart F., 1993). Ce livre avait déjà été signalé déjà dans notre chronique précédente en même temps que la thèse dont il était issu mais nous le citons à nouveau en raison de la date de sa parution.

Le rôle attribué aux femmes s'est-il modifié récemment au Rwanda? Dans le Rwanda ancien et pendant toute la période coloniale, le corps de la femme, la maison familiale et son enclos étaient régis par les mêmes règles rituelles que le royaume. Une fois mariées, les femmes devaient cultiver le champ de leur mari et limitaient leurs déplacements aux nécessités du ménage et à quelques visites à leurs parents. Pendant la deuxième république (après 1973), une forte croissance liée à l'aide étrangère (et non à un véritable développement) a provoqué un afflux d'argent dans les régions rurales qui a accru les inégalités sociales car il a profité surtout aux fonctionnaires et aux commerçants. Mais cela a entraîné aussi une certaine ouverture sur le monde extérieur. La crainte du manque de terres a changé aussi la perception de l'enfant comme force de travail pour les champs. La représentation du monde s'en est trouvée modifiée. Les événements de 1994 ont tué, semble-t-il plus d'hommes que de femmes si bien que les femmes font désormais près de $70 \%$ des chefs de ménage. Leur rôle s'est donc modifié. Cependant, dans les camps de réfugiés au Kivu, on a constaté que les hommes ont maintenu leur rôle. Deux faits sont à noter. D'une part, les femmes rwandaises ne participent pas aux activités commerciales comme les femmes d'Afrique occidentale par exemple, car elles doivent accepter la 
protection d'un homme. D'autre part, triste constatation, faut-il considérer comme une évolution vers l'égalité avec les hommes le fait que les femmes ont participé aussi activement que leurs maris aux massacres de1994 et au dépouillement des victimes? (de Lame D., 1999).

F. Bart nous donne une intéressante lecture, pratiquement un demi-siècle après sa réalisation, du livre que Pierre Gourou avait consacré à la répartition et à la densité de la population au Ruanda-Urundi. En rappelant la nouveauté de cette étude, à l'époque (c'est le premier livre que Gourou a consacré à l'Afrique) et le modèle de démarche géographique qu'il constitue, il montre ce qu'il faut retenir encore aujourd'hui de ses mises en garde, notamment sur le drainage des marais et sur les tentatives de regroupement de la population en villages (Bart F., 2000).

On trouvera la description d'une région particulièrement peuplée, le sud-ouest du Rwanda, et une vue générale de ses problèmes dans un article qui la qualifie de collines de turquoise (cette expression avait été utilisée pour dénommer l'opération militaro-humanitaire française qui a traversé cette région en 1994) (Prioul C., 1995).

Une étude portant sur le nord-ouest du Rwanda et plus spécialement sur deux secteurs représentatifs du géosystème des hautes terres de la crête Congo-Nil (Birembo) et d'un géosystème appalachien (Tare) montre comment les systèmes ruraux ont réagi face à la pression démographique: conquête des territoires marginaux, avec disparition progressive des pâturages, recul de moitié des surfaces en jachère, modification de l'éventail cultural (extension de la bananeraie, diminution de la part relative des céréales, forte progression des tubercules, patates douces, pommes de terre et surtout manioc qui est devenu le produit ayant le plus haut taux de monétarisation). Mais dès le milieu des années 80 , la disette se généralise. A partir de 1990, le recours à l'aide alimentaire devient permanent. Les ménages s'appauvrissent. La reproduction du modèle d'organisation sociale et spatiale est de plus en plus difficile, ce qui entraîne une déstructuration sociale. Malaise d'une classe d'âge masculine confrontée au manque de terre et au désoeuvrement. Les milices ont fait miroiter aux yeux de ces jeunes les terres de leurs futures victimes. Au moment où l'article a été rédigé, la situation foncière était en outre très incertaine suite au retour des réfugiés (Amelot $X$., 1997).

Deux pédologues, après avoir constaté que l'agriculture rwandaise avait atteint son point de rupture vers 1990, estiment cependant que la production vivrière pourrait encore être accrue par une utilisation plus rationnelle des terres et une spécialisation des cultures en fonction de la qualité des terres (Gallez A. et Van Ranst E., 1997).

\section{- Les revenus des paysans}

Une enquête menée en juin-août 1990 à Kirarambongo, localité du sud-ouest du Rwanda, à $30 \mathrm{~km}$ de Butare, montre que les revenus monétaires en milieu rural sont loin d'être homogènes (voir plus haut les publications de D. de Lame). Les commerçants et les fonctionnaires ont des revenus plus élevés que les agriculteurs. Parmi ces derniers, les riziculteurs (qui sont généralement jeunes) ont les revenus les plus élevés, puis viennent les caféiculteurs et enfin ceux qui pratiquent une agriculture de subsistance. II n'est pas étonnant que les paysans les mieux dotés en terres et en ressources monétaires soient aussi les plus dynamiques (Marysse S. et Ndayambaje Waeterloos E., 1993).

L'appauvrissement qu'a connu le Rwanda à partir de 1985, a été un des facteurs des événements de 1994. Le pays aurait atteint les limites de son modèle de développement et était entré dans une phase de décroissance. L'aide internationale avait eu des effets pervers en finançant plus de la moitié des dépenses du budget de fonctionnement de l'Etat. Les crises du prix de l'étain, entraînant la faillite de la société mixte d'exploitation de la cassitérite, ajoutées à celle du café ont imposé le recours au FMI et à la Banque Mondiale. L'ajustement structurel imposé par ces organismes a empêché le gouvernement de venir en aide aux paysans 
victimes de la dévaluation du café. Ainsi s'est formé un terreau favorable à une politique de violence (Marysse S., de Herdt T. et Ndayambaje E., 1994).

Un aperçu général des contraintes naturelles et techniques auxquelles doivent faire face les paysans rwandais aboutit à la conclusion que la majorité de ceux-ci sont acheteurs nets de produits vivriers alors que les emplois non agricoles sont rares. Les problèmes que les paysans considèrent comme les plus importants sont la faible fertilité des terres, le risque de pluies insuffisantes, le manque de terre et d'engrais. Leur situation ne pourra être améliorée sans un ensemble complexe de mesures à court et à moyen terme requérant un partenariat équitable de la part des pays industrialisés (Ngwabije, 1994).

\section{Burundi}

Contrairement à l'image que l'on a généralement du paysan burundais, celle d'un défricheur acharné, déclenchant l'érosion des sols par sa culture des fortes pentes, il serait en fait très soucieux de la gestion et de la conservation de la biomasse. Un article en donne quelques exemples. II faudrait aider le paysan en lui fournissant les moyens nécessaires (Cochet $\mathrm{H}$., 1996). Le même auteur a consacré une thèse d'agronomie sur le devenir de l'agriculture et des agriculteurs burundais. II défend l'hypothèse d'une capacité endogène de la petite paysannerie à se développer et à accumuler. II définit un modèle d'évolution du système dominant qu'il nuance en fonction des particularismes régionaux. Le dernier chapitre de sa thèse est une critique des rôles de l'Etat et de l'encadrement technicien et insiste sur leur responsabilité majeure dans la crise actuelle (Cochet $H_{\text {., }}$ 2001). On trouvera un bref compte rendu de cette thèse dans Charlery B. (2003), revue Autrepart, 27, pp. 133-143. $\mathrm{H}$. Cochet, également, dans deux articles publiés en 2000 et en 2001, s'interroge sur la diversité et l'âge des paysages agraires du Burundi. II propose d'abord de reconnaître au Burundi au moins sept grands paysages agraires: I'Imbo (région basse au fond du fossé du lac Tanganyika), les Mumirwa (escarpement occidental), les hauts versants abrupts du Mugamba-nord, le paysage des «mille collines» des plateaux centraux, le sudest (Kirimiro, Buyogoma, est du Bweru et sud du Bugesera), les franges périphériques de basse altitude (Kumoso, sudest du Bweru, nord du Bugesera). II s'interroge aussi sur l'ancienneté des traits constitutifs de ces paysages. La forte couverture arbustive, par exemple de Grevillea, est récente, de même que les innombrables parcelles de caféiers. La grande extension des bananeraies ne commence pas avant le début du XXe siècle. Récente aussi la place importante occupée par le taro (sous les bananiers), la patate douce, les pommes de terre, le manioc, qui étaient presque inexistants voici un peu plus d'un siècle, quand sorgho, maïs, éleusine et haricot constituaient les cultures et l'alimentation de base. Et maïs et haricots ne remontent pas non plus très loin dans le temps. «Le système agraire d'aujourd'hui apparaît ainsi comme éminemment moderne». Le Burundi a donc connu deux révolutions agraires majeures; l'une qui correspond à l'adoption du maïs et des haricots (XVIIe et XVIIIe siècles), l'autre qui correspond à la période coloniale. Les plantes cultivées ont changé avec les systèmes d'assolement. II y a eu récupération des déjections nocturnes des bovins, mise en valeur des bas-fonds, etc., mais l'outillage technique est resté ce qu'il était depuis la toute première révolution de l'introduction du fer. L'auteur, s'il reconnaît que la grande extension de la bananeraie pour la bière représente une déperdition alimentaire certaine, fait remarquer aussi que la bananeraie produit une biomasse importante, se substituant ainsi à la fumure du bétail dans la reconstitution de la fertilité du sol et fournit aux paysans des ressources monétaires (Cochet H., 2000).

Au Burundi encore, dans les Mwira, versant escarpé oriental du fossé du lac Tanganyika, les fortes pentes, l'intensité des précipitations, la baisse de la température (au dessus de 2000 m, le bananier donne mal, de même que la patate douce et les haricots au dessus de $1900 \mathrm{~m}$ ), la pression 
démographique (300 et parfois 400 habitants au $\mathrm{km}^{2}$ ) sont des contraintes très fortes et nécessiteraient, outre une politique de conservation et de restauration des sols (les techniques de labour favorisent l'érosion), des opérations de reboisement (les feux pour l'aménagement de pâturages ont détruit le couvert végétal) et une diversification des activités économiques. L'exode rural vers Bujumbura est d'ailleurs important (Nkurunziza F.-X., 1996).

Une étude d'économie agricole, pendant une durée de cinq ans, de 58 exploitations des deux collines voisines de Campazi et Muruta, sur le versant oriental de la crête Congo-Nil (1800 à 2000 m d'altitude), où la population est passée de 398 habitants au km² en 1965 à 613 en 1990, montre que les paysans burundais se sont intégrés de plus en plus à l'économie de marché en étendant les cultures de rente (café, thé, tabac, pomme de terre), en abandonnant partiellement les cultures vivrières traditionnelles (sorgho, éleusine), en recherchant du travail salarié en dehors de l'exploitation. L'analyse de photos aériennes de 1959, 1975 et 1984 a permis de mesurer par exemple le recul des pâturages (13 à $15 \%$ par an) et la disparition des jachères de longue durée. Cependant l'agriculture reste dans une logique de subsistance avec tout compte fait de simples ajustements pour maintenir les équilibres fondamentaux alimentaires, monétaires et de fertilité des sols. Mais le système atteint ses limites. Très curieusement rien n'est dit sur la culture de la banane, ni sur la production de bière. Serait-ce que l'altitude est trop élevée pour cette production? (Verhaegen E. et Degand J., 1993).

Une grosse enquête franco-burundaise a porté sur les régions du sud-est du Burundi, le Buyogoma et le Kumoso, qui se comportent comme une périphérie dans le système spatial du pays (Gahama J. et Thibon C., 1994). La première partie s'interroge sur les aspects répulsifs qu'avaient les deux régions: perception d'un environnement malsain, d'espaces peu peuplés, hantés par les esprits et les sorciers. Cet ensemble se tournait davantage vers le Buha (Tanzanie) que vers le reste du Burundi. La deuxième partie traite la démographie du front pionnier et la structure des exploitations, la troisième, les contacts, les échanges et les mutations auX XIXe et XXe siècles. On retiendra notamment les communications sur l'évolution des paysages et le développement agricole, avec des cartes donnant des exemples de paysages agricoles de montagne, de haut plateau, de vallée d'altitude, de crête, etc. (Ndayirukye S., 1994), sur les densités de population dans leurs relations avec l'occupation du sol et sa fertilité (Bidou J.-P., 1994 a), sur les types d'exploitations agricoles à partir d'une analyse en composantes principales (Bidou J.P., 1994 b) et sur la mise en valeur des marais (Nsabimana T. et Manirakisa, 1994).

La guerre civile a ruiné l'économie du Burundi. Cependant l'agriculture vivrière a permis la subsistance d'une population particulièrement dense. Les recettes des cultures de rente sont en forte baisse. L'auteur de l'article envisage les possibilités de sortir de la crise (Cazenave-Piarrot A., 2005).

\section{L'emprise du café}

L'importance du café dans l'économie des hautes terres est décrite dans un ouvrage consacré à cette question en Afrique et en Amérique latine (Tulet J.-Ch., Charlery B., Bart F. et Pilleboué J., 1994). $F$. Bart analyse plus particulièrement le cas du Rwanda où les structures de production sont atomisées (les parcelles individuelles de café ont de 7 à 8 ares seulement). La culture, encouragée ou même souvent imposée par le pouvoir colonial puis par la suite par le pouvoir national, se fait en dessous de l'altitude de $2000 \mathrm{~m}$. Deux régions principales entre 1400 et 1900 m d'altitude: le Plateau central et les régions riveraines du lac Kivu. Le café est le principal pourvoyeur de revenus monétaires pour le paysan et de devises pour l'Etat. D'où la forte vulnérabilité des exploitations paysannes et de l'économie rwandaise en général aux crises des prix, particulièrement à la crise de 1985 (Bart F., 1994). La croissance démographique et la remise en cause de la sécurité alimentaire compromettent le fonctionnement du système et entraînent l'affaiblissement de l'Etat (Uwizeyimana L., 1996 et 2001). 
Au Burundi, où la culture du café avait été rendue obligatoire dès 1931, l'Etat, largement tributaire du café pour ses ressources en devises, exerce sa mainmise sur la filière. Comme dans les régions caféières, qui sont aussi les régions les plus densément peuplées, la production vivrière est devenue souvent déficitaire, beaucoup de paysans comptent sur le café pour couvrir leurs dépenses alimentaires. Il est possible d'étendre la culture dans certaines régions ou d'accroître les rendements. Mais dans certaines parties du pays, l'extension de la caféiculture menacerait la sécurité alimentaire (Bidou J.E., 1994C).

Dans le Kivu, au Congo, les plantations européennes de café qui avaient été créées avant 1960, ont été pour la plupart abandonnées par leurs anciens propriétaires. Certaines avaient été reprises par des barons du régime mobutiste. Dans les années 80, la production est désormais essentiellement assurée par les paysans (67000 ha, 140000 planteurs). Le café, en 1988, était le deuxième produit d'exportation du Congo-Zaïre et faisait $85 \%$ des recettes d'exportations de produits agricoles. La contrebande à l'exportation aurait porté sur 10 à $30 \%$ de la récolte (Bushoki Batabiha J., 1994). Dans leur introduction à l'ouvrage collectif dans lequel cette étude est publiée, les éditeurs affirment que le Kivu est le type même de la montagne coupée du monde extérieur à la fois par la distance, l'insécurité et la faillite du système de transport. Le propos est quelque peu excessif mais il est vrai que la caféiculture y est pratiquement abandonnée à elle-même, enclavée dans une région aux infrastructures déficientes et tributaire de circuits parallèles de commercialisation passant par les pays voisins. Cela avait été écrit avant les événements de 1994 et la série de guerres intérieures. La situation n'a donc pu s'améliorer.

\section{La question de l'habitat dispersé}

Depuis l'époque coloniale, on s'interroge sur les avantages et les inconvénients de l'habitat dispersé qui caractérise les campagnes rwandaises. Pour une meilleure fourniture des services à la population, on a préconisé souvent de regrouper les enclos. La plupart des tentatives en ce sens avaient échoué. Après 1994, diverses opérations d'installation de villages groupés ont été effectuées comme mesures d'urgence. II s'agissait de donner un toit à des gens qui n'en avaient pas comme les exilés tutsi revenus au pays ou les réfugiés hutu rentrés du Congo et dont les maisons avaient été détruites ou occupées par des Tutsi. Parfois on a voulu regrouper des populations hostiles au nouveau pouvoir pour mieux les surveiller. Des opérations du même type ont visé la colonisation de l'est du pays. D'autres ont répondu aussi à un objectif de développement (points de fixation d'un éventuel exode rural) et à un objectif de réconciliation nationale. L'auteure de l'article a recherché l'avis des habitants concernés. Celui-ci a été majoritairement positif. Elle s'est interrogée sur l'avenir et les possibilités de généralisation de ces opérations à l'ensemble du pays (Imbs F., 2000). On a vu plus haut que des opérations du même type avaient été effectuées au Burundi.

Les fortes densités rurales et la capacité des terroirs

Les fortes densités agricoles atteintes sur des surfaces du Kivu d'altitude ont conduit des pédologues à calculer les potentialités de quatre surfaces-tests de $2500 \mathrm{~km}^{2}$ chacune (Lubero, Butembo, Masisi et Sud-Kivu au nord-ouest de Bukavu). Pour chaque échantillon, la surface cultivable est déterminée en fonction des sols et du relief. La production potentielle par le système traditionnel est calculée en tenant compte de la durée des jachères et des disponibilités en maind'oeuvre. La densité potentielle est calculée sur la base d'une ration alimentaire de $2200 \mathrm{kcal}$ par personne. C'est dans l'échantillon de Masisi que, grâce à la présence d'un saupoudrage de cendres volcaniques, les meilleurs résultats peuvent être obtenus en culture traditionnelle (297 habitants au $\mathrm{km}^{2}$ ). L'intensification de l'agriculture apparaît indispensable pour 
résoudre les problèmes des surfaces qui ont déjà plus de 300 habitants au $\mathrm{km}^{2}$ et sur lesquelles les paysans cultivent, sans mesures de protection, des pentes supérieures à 70 \% (Sys $\mathrm{C}$. et Van Ranst $\mathrm{E}$., 1996).

Les pédologues offrent de nouveaux instruments pour la définition d'une politique agricole au Rwanda. En numérisant les 43 cartes topographiques du pays au 1/50 000 et les cartes pédologiques correspondantes et en les connectant à une base de données des profils géologiques, on peut, en appliquant les techniques des systèmes d'information géographique, (SIG) établir de nombreuses cartes thématiques (classification des terres, terres irrigables, vulnérabilité des sols, etc.). L'article en donne quelques exemples (Imerzoukene S. et Van Ranst E., 2001). Voir aussi sur le même sujet l'article de Verdoodt A. et Van Ranst E. (2006).

Divers auteurs préconisent une agriculture plus "écologique» au Rwanda car ils considèrent que le modèle d'agriculture appliqué depuis 1970 a échoué à résoudre les problèmes de surpopulation et de dégradation du sol. Deux d'entre eux préconisent d'associer le fumier à des engrais minéraux comme le travertin et les cendres volcaniques pour maintenir la fertilité du sol (Stache N. et Drechsel P., 1997).

\section{Eau et aménagement}

Un colloque réunissant des enseignantschercheurs des départements de géographie de I'Université du Burundi, de I'Université Nationale du Rwanda et de I'Institut Supérieur Pédagogique de Bukavu s'est tenu en mai 1991 à Bujumbura sur le thème L'eau et l'aménagement dans l'Afrique des Grands Lacs. Dix-neuf communications ont traité de l'évaluation des ressources en eau et de leur aménagement, de l'aménagement des marais, de l'approvisionnement en eau potable, de l'exploitation des ressources halieutiques, de l'hydroélectricité, des transports lacustres, etc. (Sirven P., éd., 1993). Ce sera le dernier grand colloque scientifique organisé sous la coupole de la coopération française dans ces régions, qui cessera d'y fonctionner après 1994. II a traité notamment la question de l'approvisionnement en eau potable dans les espaces ruraux qui reste un problème général de santé publique. D'une part, il y a toujours le problème de la corvée d'eau. D'autre part, le stockage est une source de contamination (Gatera F., 1993). Au Burundi, on a mis la priorité sur l'alimentation en eau potable des centres hospitaliers en espérant une généralisation ultérieure de l'opération (Ndayishmiye J.-P., 1993). Par défaut de maintenance, de nombreux aménagements ne sont plus utilisables. Dans ce dernier pays cependant, vers 1990, un quart de la population rurale est desservie en eau potable. (Nsabimana S., 1993 b). Dans la préfecture de Ruhengeri, au Rwanda, où les pluies sont abondantes et les sources nombreuses, la population peine à maîtriser l'érosion des versants et les inondations tandis que les habitants des sommets souffrent d'une pénurie d'eau (Nzamwita D., 1993).

Les mêmes problèmes se posent, on s'en doute, dans les provinces congolaises voisines du Kivu, au Congo. A Bukavu, le ravitaillement en eau potable suit malaisément la croissance rapide de la ville et souffre de la vétusté du réseau ainsi que des carences dans le traitement des eaux (Muzalia Wakyebwa K., 1993). Dans le Bwisha, région très peuplée coincée entre le parc des Virunga et la frontière du Rwanda et de l'Uganda, de grands projets d'adduction d'eau étaient en cours de réalisation avant 1994. Trois petites centrales hydro-électriques avaient été installées dans le sud de cette région (Ilunga L., 1993). De même, dans la province du Sud-Kivu, la plaine de la Ruzizi a des pluies peu abondantes mais peut disposer de grandes ressources en eaux fluviales. De grands aménagements hydrauliques pour l'irrigation et la production d'électricité ont été réalisés à la fin de la période coloniale et par la suite. Plusieurs ne fonctionnent plus (llunga $L$. et Londa L., 1993). La Région des Grands Lacs est propice à divers aménagements hydroélectriques. Certains barrages peuvent jouer un rôle régional, en animant des pôles de développement, comme celui sur la Ruzizi, ou pourraient le jouer lors- 
qu'ils seront réalisés, comme celui de Rusumo sur la Kagera. De petits barrages locaux fonctionnent également au Rwanda, au Burundi et au Kivu mais les réseaux sont mal ou peu connectés (Ndayirague G., 1993).

Les changements dans les fonds de vallée et les régions basses

\section{- Les situations anciennes}

Alors que l'histoire traditionnelle de l'Afrique des Grands Lacs est généralement associée aux complexes agricoles et pastoraux des collines et des plateaux, J.-P. Chrétien insiste sur l'importance qu'avaient les régions basses, rives des lacs, fonds marécageux des vallées, avant la fin du XIXe siècle. C'est là qu'on produisait les poteries, le sel, qu'on fabriquait les pirogues. On y pratiquait la pêche beaucoup plus qu'on ne l'a souvent prétendu. Des bas-fonds étaient aménagés en plates-bandes séparées par des canaux. Ces techniques d'irrigation se seraient même développées encore pendant le XIXe siècle. II y avait une véritable civilisation «aquatique». Mais les marques de celle-ci auraient été effacées par le dépeuplement causé à la fin du XIXe et au début du XXe siècle par la maladie du sommeil (Chrétien J.-P., 2007). Un géographe burundais rappelle d'ailleurs que, d'après les récits des premiers Européens qui visitèrent le Burundi, les marais étaient exploités pour l'agriculture, l'élevage, la chasse. II retrace l'évolution de leur exploitation pendant l'époque coloniale et postcoloniale (Nsabimana T., 1993a).

\section{- L'aménagement récent}

Dans le Buyenzi, région très peuplée du nord du Burundi, les marais, qui étaient utilisés pour des cultures de saison sèche mais restaient inondés le reste de l'année, ont été transformés en rizières depuis 1980. Mais la production a été affectée à plusieurs reprises par des maladies du riz et parfois par la mévente du produit (Bidou J.E., 1993).

Le sud-est du Rwanda a les pluies les plus faibles du pays et est aussi peu peuplé. Son histoire tectonique et géomorphologique y a créé de vastes étendues de lacs et de marais. Le gouvernement y a donc encouragé des mouvements de colonisation à partir des régions très peuplées. Cela ne s'est pas fait sans conflits ente les «autochtones» et les immigrants (Barambirwa J.B., 1993).

Des marais ont été mis en valeur aussi dans le Kivu voisin, non loin de Bukavu, depuis 1950. Ils donneraient des résultats convenables pour les cultures maraîchères (Sebakunzi Ntibibuka et Birembano Bahati, 1993)

De nombreux projets combinant pisciculture et utilisation agricole des marais continuent à être exécutés au Rwanda. Un colloque leur a été consacré à Butare, en 2001. La plupart des communications préconisent le recours à des systèmes agro-piscicoles inspirés de ceux qui sont pratiqués au Mexique et en ExtrêmeOrient et plusieurs insistent sur l'intérêt du riz irrigué (ou inondé) d'autant plus que le riz semble bien accepté par toutes les couches de la population. II est recommandé que toute opération soit précédée d'une étude d'incidence et de faisabilité conduite par une équipe multidisciplinaire et que les communautés locales soient associées à toutes les phases du projet. Un objectif prioritaire est la réhabilitation de marais aménagés mais actuellement dégradés. Les marais de haute altitude doivent être préservés car ils constituent des châteaux d'eau (Kanangire C.K., Micha J.C., Gashagaza J.-B.M., 2001).

La pisciculture avait démarré au Rwanda, dans les années 40, avec trois espèces de Tilapia. Depuis les années 80, on a eu recours à des souches plus résistantes aux basses températures, ce qui permet de pratiquer leur élevage jusqu'à 2200 m d'altitude. Depuis 1989, le Tilapia est associé au Clarias. L'association de ces espèces complémentaires en ce qui concerne leur alimentation, donne des rendements supérieurs de $50 \%$ à ceux de l'élevage d'une seule espèce. Dans les stations piscicoles gouvernementales, on élève en même temps des porcs, des poules, des canards, des lapins, des chèvres et des moutons. Cette formule 
est préconisée pour résoudre les problèmes de l'alimentation paysanne (Verheust J., Rurangwa E. et Ollevier F., 1995).

\section{- La pêche dans les lacs}

La pêche artisanale dans le lac Tanganyika au Burundi s'est développée surtout après 1950. Ses récoltes sont écoulées principalement à Bujumbura (Ndayirukiye S., 1993). La pêche est active aussi dans le lac Kivu. A Kalengera, près de Bukavu, une centaine de familles vivent uniquement de la commercialisation des produits de leur pêche. Mais elles devraient être mieux encadrées (Kanyingini Mwenyimali, 1993). Comme, dans ce lac, le milieu est anaérobique en profondeur, la productivité est plus grande près du littoral que dans la zone pélagique. L'introduction de la sardine Limnothrissa miodon avait permis d'augmenter les ressources de la pêche. On a introduit de nouvelles méthodes dont celle au filet maillant. On a déterminé la dimension optimale des mailles de celuici mais il faudrait procéder à un survey scientifique de la masse des poissons pour éviter toute exploitation exagérée (Kanyingini B. et al., 1997).

Dans les lacs du Rwanda (Muhazi et Mugesera), à la fin des années 1980, on a introduit un Protoptère. Mais comme celui-ci, qui consomme des mollusques gastéropodes, consomme aussi des alevins de Tilapia, il faudrait un suivi pour vérifier s'il ne perturbe pas la communauté ichthyologique actuelle (Micha J.C. et Gashagaza J.-B., 2002).

\section{LES PROJETS DE DÉVELOPPEMENT AGRICOLE ET RURAL}

Sur les interventions de l'Etat en matière agricole du début de la période coloniale jusqu'à la fin du XIXe siècle (notamment les cultures obligatoires, les corvées imposées aux paysans, les plantations en collaboration, les paysannats, les coopératives, etc.), on lira le livre de J. Mokili (1998).

A l'occasion d'une présentation de l'ouvrage de la Fondation Roi Baudouin, dont il a été le principal coordinateur "Développement rural en Afrique centrale 1910-1960/62» et dont nous avons rendu compte dans notre chronique précédente, V. Drachoussoff rappelle que le monde rural de l'Afrique centrale est confronté aujourd'hui à cinq impératifs: survie, déblocage et relance, recherche, développement, protection de l'environnement. II estime qu'on ne pourra y répondre efficacement qu'en commençant dans un cadre régional ou local progressivement étendu en gardant toujours à l'esprit que c'est aux Africains qu'il importe d'élaborer leur type de société moderne (Drachoussoff V., 1994).

Voici un exemple de reconversion qui semble avoir réussi; celle de Yuki, sur la rive droite du Kasai, à $110 \mathrm{~km}$ en aval d'llebo. Jadis poste à bois pour les steamers, il s'est reconverti, à partir des années 50 en exploitation forestière envoyant à Kinshasa ses grumes et aussi du charbon de bois produit dans des fours traitant les déchets. Dans les années 80, ouverture d'une petite piste d'aviation, création d'une école, établissement de plantations de caféiers dans les espaces déboisés avec des cultures vivrières sous les arbustes. Indice d'une augmentation du niveau de vie: le taux de natalité aurait diminué fortement. La population a changé d'attitude envers son environnement forestier et accepte des mesures de protection (Paelinck H., 2004).

La population des campagnes affirmerait aujourd'hui de plus en plus sa volonté de se prendre en charge. D'où l'abondance des projets de développement issus des communautés de base et soutenus par des ONG (zaïroises à l'époque de l'article). En 1993, 64 \% des organismes travaillant en milieu rural sont laïques, $36 \%$ confessionnels (parmi ceux-ci, $48 \%$ catholiques, $42 \%$ protestants, $10 \%$ kimbanguistes et autres). Cependant, malgré le nombre croissant des projets, la paupérisation croît ainsi que la part de la population marginalisée. Outre les causes générales (détérioration du contexte politique et socio-économique), des facteurs d'explication doivent être recherchés dans un mauvaise ou une insuffisante prise en compte des conditions du milieu, dans l'amateurisme 
des promoteurs, dans la non-participation des communautés et des populationscibles à la conception et parfois à la gestion du projet, dans la persistance des contraintes socio-culturelles, dans les lacunes du partenariat nord-sud (relations de subordination, imprécision du rôle de chaque partenaire, absence d'actes juridiques dans les accords de financement) (Makwala ma Mavambu J., 1995). On trouvera dans un autre article, d'autres réflexions sur cette question et notamment sur les effets de l'arrêt de financements extérieurs qui a entraîné l'abandon de réalisations en cours (Lubana Ngiyenze Amena, 1992).

On aura trouvé des exemples de projets de développement rural inspirés et gérés par des ONG dans les collines rwandaises étudiées par D. de Lame et F. Migeotte dont il a été question plus haut. De façon générale, on peut dire que les ONG travaillent avec enthousiasme mais aussi parfois avec une certaine ambiguïté qui expose à quelques dérapages. On a fêté, en 1995, à l'Université de Gand, le 25e anniversaire de l'une d'entre elles, le Centre de Développement Intégral de Bwamanda (C.D.I. Bwamanda), non loin de Gemena (Equateur). Conçue au départ, à la fin des années 60, par un missionnaire et des médecins pour faire participer la population à un programme de santé publique, elle a cherché ensuite à améliorer les revenus de cette population pour qu'elle puisse subvenir elle-même à un programme social. Elle a mis sur pied une mutuelle d'assurance-santé. D'abord centrée sur le développement de la production vivrière, elle a aidé, pour accroître les revenus monétaires, à développer la culture du café et assurer sa commercialisation, sous la forme d'une production éthique avec le label Max Havelaar. Etendant son aire d'action à une population de près de 500000 habitants, elle a pu être considérée comme un véritable modèle. Mais elle a dû aussi s'occuper des systèmes de communication nécessaires à l'exportation de la production. Devant la déficience de l'Etat, elle doit entretenir 400 $\mathrm{km}$ de pistes (en signant des conventions avec l'Office des Routes) et construire des ponts. Elle a constitué une petite flotte de remorqueurs-pousseurs et de barges pour assurer le transport jusqu'à Kinshasa. Elle a créé aussi diverses structures de conditionnement des produits dans l'Ubangui et à Kinshasa. Bref, elle a diversifié ses activités et est devenue une véritable entreprise (Zana Aziza Etambala et Marysse S., 1995). Elle a même envisagé de s'étendre au Bandundu en prenant en charge (ou en parrainant) la reprise par une communauté locale d'établissements huiliers appartenant à une firme d'origine portugaise. Suite à une inspection, le gouvernement congolais a estimé en 1997 qu'elle ne pouvait plus être considérée comme une association sans but lucratif mais comme une société privée, et donc soumise à la législation sur le personnel salarié. Le cas du DPP (Développement Progrès Populaire) d'Idiofa, au Kwilu, est une histoire du même type. Au départ, dans les premières années d'indépendance, petite coopérative créée par un missionnaire et une personnalité locale à Mateko, cette ONG s'est étendue à tout le diocèse d'Idiofa, aménageant des structures d'animation rurale et de développement communautaire. Considérée longtemps, elle aussi, comme un modèle, elle a dû assurer le transport de ses produits vers Kinshasa, construire des ponts, ouvrir ou rouvrir des routes. La dispersion de ses efforts l'a menée, semble-til, au bord de la faillite en 1996 (M'Pene Ngaluley Z., 2002). D'autres ONG, trop étroitement associées à la personne de leur fondateur (souvent un missionnaire) et parfois dirigées par lui de façon autoritaire, n'ont pas survécu au départ de celui-ci (voir l'exemple cité dans l'article de F. Migeotte).

Une conséquence inattendue d'un projet de développement est la propagation de rumeurs débouchant sur des psychoses et allant jusqu'à perturber la vie sociale des régions rurales. Après la rébellion muleliste, l'association Mouvement Progrès Populaire (MPP), qui donna par la suite naissance au DPP Idiofa dont il a été question dans le paragraphe précédent, a été créée par un missionnaire européen et un enseignant congolais. Elle a introduit dans le diocèse de Mateko (nord-ouest du territoire d'Idiofa) l'élevage bovin, au cours de la seconde moitié de 
la décennie 1960. Aussitôt une rumeur a circulé dans les villages affirmant que des mitumbula, c'est-à-dire des ravisseurs à la solde de certains Blancs, capturaient des Noirs pour les transformer, dans des laboratoires, à la suite d'injections diverses et d'un régime alimentaire particulier, en vaches destinées à la production de viande. Au départ, on situait le laboratoire à Kinshasa ou dans les grandes villes minières, puis ce fut dans le centre de formation d'éleveurs et d'agents vétérinaires, dans le diocèse même. Une des causes de ce «malentendu» a été sans doute l'absence de toute participation de la population à la définition et à la mise en oeuvre du projet, qui a été perçu comme une nouvelle manifestation de Blancs (le MPP était même surnommé le Mouvement des Bapères, c'est-à-dire des missionnaires blancs). L'auteur aurait pu rappeler que la rumeur dont il parle n'a été que la réactivation d'une rumeur ancienne (notamment pendant la dernière guerre mondiale) selon laquelle les Blancs s'emparaient la nuit de Noirs sur les routes pour en faire du corned beef (Tshiala L., 2000).

Au moment de la Conférence nationale souveraine, on a pu considérer que les ONG d'origine nationale allaient contribuer à la formation d'une société civile. Une étude portant sur les ONG du SudKivu présentait ce processus comme en marche avant 1994. Une des premières ONG avait été le Comité Anti-Bwaki (1982-1983) qui comportait une prise en charge par des communautés locales de leurs problèmes de malnutrition et de façon générale de leurs problèmes sanitaires. Deux Instituts supérieurs à Bukavu avaient fourni les cadres de ces mouvements qui s'étaient structurés au niveau régional. Grâce à cette structure, les ONG du Sud-Kivu ont donc pu participer à la Conférence nationale souveraine. Une plaquette qui expose les péripéties de cette participation est brièvement résumée dans une note de la revue ZaïreAfrique (Kikassa F., 1995). Mais nous ne savons pas quel aura été le rôle de ces ONG dans la période de troubles et de guerres que le Kivu a connue peu après. Sur le rôle des ONG pour la formation d'une société civile en milieu urbain, voir le chapitre "Géographie urbaine».

\section{LE DÉVELOPPEMENT RÉGIONAL}

Beaucoup de chercheurs se sont intéressés au développement régional. L'un d'eux montre que les projets de développement du Katanga doivent prendre en compte les spécificités des sous-régions qu'il y a reconnues. II analyse les grands projets agricoles qui ont été entrepris dans ces différents ensembles, à la fin des années 70 ou dans la deuxième partie de la décennie 80 (Bushabu Mbengele-Ming, 1998).

Dans le Bulletin Géographique de Kinshasa, plusieurs articles (datés de 1992 mais ils avaient été en fait publiés après notre chronique antérieure qui ne les a donc pas cités) traitent de questions analogues. L'un cherche à définir, pour toute la partie sud du pays, du BasCongo au Katanga, les régions prioritaires pour des projets de développement rural sur base de critères de population, de production agricole et de l'existence éventuelle d'organismes de développement (Mlibwa Wazye, 1992). Un autre énumère les données disponibles sur les régions riveraines du Kasai dans la province de Bandundu et propose des thèmes d'études (Noti N'Sele Zoze, 1992). Un autre encore s'interroge sur les stratégies spatiales à mener dans le cadre du développement régional, analysant quelques grandes opérations, insistant sur les faiblesses des petites, proposant d'évaluer le développement régional à partir de groupes-cibles, de diviser le pays en compartiments d'aménagement et préconisant l'autonomie régionale dans le cadre d'un système fédéral (Mashini, 1993). Les espoirs qui ont été mis dans une décentralisation territoriale des responsabilités ont été déçus. La politique de décentralisation des dernières années de l'ère mobutiste a été à peine amorcée. Elle n'a créé qu'une dualité de pouvoirs et ne s'est accompagnée d'aucune stratégie de développement. Le cas du Kwango-Kwilu est pris comme exemple (Mashini 1995).

Le Bas-Uele a été affecté fortement par la 
révolte dite des Simba (1964-1966) qui a détruit une grande partie de ses infrastructures et provoqué de grands massacres. La remise en ordre de la région a été gênée par les interventions bureaucratiques du parti MPR puis par la zaïrianisation. Depuis 1980, cette sous-région est négligée de plus en plus par le pouvoir central. La libéralisation de l'extraction minière, c'est-à-dire ici surtout de l'or, a détourné les paysans, les élèves des écoles et le personnel enseignant de leurs activités normales et a conduit finalement à davantage de précarité (Mily Denda-Sakala D., 1996).

Nous avons rappelé comment les constructions régionales se font et se défont en fonction des évolutions économiques et politiques. Le Kwilu, que nous prenons en exemple, s'est développé pendant la période coloniale comme un espace producteur d'huile de palme, sous le contrôle de grandes sociétés huilières. Cette économie s'est fortement dégradée depuis 1960. Les Plantations Lever (exH.C.B.) ont même revendu des usines et des plantations à des hommes politiques (en même temps hommes d'affaires) locaux. L'ouverture d'une route macadamisée a permis à la région de devenir un important bassin d'alimentation de Kinshasa, surtout pour les cossettes de manioc. La dégradation ultérieure de cette route a rendu quelque vigueur aux transports fluviaux (organisés par des entrepreneurs privés). Le Kwango-Kwilu est devenu une «périphérie» de Kinshasa. Hommes d'affaires et commerçants de la capitale contrôlent désormais une part importante de ses activités. L'article fait allusion à une activité qui s'est développée fortement après la date de sa publication: les trafics transfrontaliers portant sur le diamant de l'Angola et même l'extraction de diamant sur le territoire congolais, notamment dans la vallée du Kwango (voir notamment les articles de F. De Boeck et de Maboloko cités plus loin) (Nicolaï H., 1996b).

Le Kasai est un autre exemple de restructuration du territoire. Le processus est bien décrit dans un article de Kabamba. Déjà recentré autour de Luluabourg (actuel Kananga) après la construction du rail
B.C.K., le Kasai a connu des tensions ethniques en 1959-1960 entre Luluwa et Luba qui ont contraint ces derniers à se replier sur leurs territoires d'origine dans l'actuel Kasai oriental. II a vu le retour d'une partie de ses ressortissants (près de 100 000) installés dans les régions minières du Katanga lors de la sécession de cette province en 1960. De 1991 à 1994, c'est près d'un demi-million de Kasaïens qui ont été «refoulés» du Katanga, dans une seconde vague «d'épuration ethnique». En 1960, le Kasai était un espace essentiellement agricole, fournissant en produits vivriers le Katanga industriel, avec deux centres d'exploitation du diamant (Tshikapa et Bakwanga). Depuis lors, le Kasai a connu l'effondrement de son économie agricole, la crise de ses transports terrestres (rail et route), la disparition des paysannats cotonniers de Ngandanjiga, la libéralisation de la production de diamant, une croissance importante de sa population urbaine (aujourd'hui $35 \%$ de la population totale) dont la grande agglomération de Mbuji Mayi, coeur de la région diamantaire, et de nombreuses villes, petites ou moyennes. Actuellement l'espace est dominé par la région du triangle KanangaMbujimayi-Mwene Ditu où se trouvent les pôles d'encadrement politico-administratif des deux provinces du Kasai occidental et du Kasai oriental, les principaux équipements socio-économiques, les principaux foyers de production artisanale du diamant. Au sud-ouest, le pôle diamantaire secondaire de Tshikapa. Le nord, couvert par la grande forêt, est resté en dehors de l'évolution économique générale et toutes ses activités agricoles sont en déclin (Kabamba K., 2002). L'auteur, qui s'intéresse au concept de territorialité, a consacré une thèse de doctorat aux relations à la ville dans la campagne environnante de Kananga. II part de l'idée que, pour les paysans, la ville fait partie d'une aire d'appropriation qui permet la satisfaction des besoins de survie face à la crise et qui se base sur un sentiment d'appartenance ethnique, la ville étant considérée comme lieu d'identité dans les représentations et les imaginaires. La relation à la ville est aussi une relation au pouvoir (Kabamba K., 2000). 


\section{LES PROBLÈMES ALIMENTAIRES}

Un colloque a développé le thème des relations entre l'alimentation, les traditions et le développement en Afrique tropicale (Devisch R., De Boeck F., Jonckers J., 1995). Après un long chapitre introductif (De Boeck F. et Devisch R., 1995), plusieurs communications concernent les territoires qui nous intéressent.

Celle qui traite des Zande s'occupe davantage de leurs traditions agricoles que de leurs problèmes alimentaires. Les Zande réfutent la qualification de nomades qui leur a été parfois attribuée parce qu'ils se déplaçaient à travers trois frontières. C'était en fait surtout pour échapper aux impôts et corvées. Ils vivaient dispersés dans un réseau inextricable de chemins. Les autorités coloniales voulurent mettre de l'ordre dans cette "sauvagerie». Au Soudan, dans le cadre du Zande Scheme (pour la culture du coton), les autorités anglaises ont disséminé les exploitations loin des routes parce qu'ils considéraient que c'était dans la nature des Zande. Sur le territoire de l'actuelle RCA, les Français, au contraire, les ont rapprochées des routes, parfois par la force. Les politiques agraires coloniales, comme les projets de développement aujourd'hui, ont mal compris les interdépendances entre la culture de rapport et le contexte des pratiques et des croyances culturelles et ont abouti souvent à des répercussions qui n'avaient pas été prévues (Grootaers J.-L., 1995). Hélène Pagézy, spécialiste de l'alimentation des Ntomba, qui habitent les forêts inondées, autour du lac Tumba, récapitule les résultats de ses études dans une communication très fouillée. Les villages et les champs des Ntomba sont installés sur les parties hautes. Les agriculteurs (des Oto) vivent en relation étroite avec les Twa. Le régime alimentaire est à base de manioc et de produits animaux avec une sauce d'huile d'Elaeis. Pendant les saisons sèches (deux fois par an), les Oto installent des campements de pêche sur les rivières, les Twa leur fournissent du bois pour le fumage du poisson. Pendant ce temps, les femmes restées au village s'occupent des champs puis rejoignent les maris. Cette période des campements, pendant laquelle l'alimentation est riche en protéines, permet aux villageois de récupérer leur capacité biologique. Pendant la période des pluies, les protéines sont surtout fournies par la chasse mais celle-ci est aléatoire. La grande saison des pluies est vécue comme une saison de «faim». On retiendra les rituels particuliers concernant les femmes primipares. Pendant un an ou plus, elles sont exemptées de tout travail agricole (et même domestique). Les relations sexuelles leur sont interdites pendant trois ans, d'où l'espacement des naissances. Ces jeunes mères, à peine sorties de l'adolescence, peuvent ainsi achever leur croissance tout en portant puis en allaitant leur enfant. Tout ce système est cependant en voie de dégradation et des formes de malnutrition apparaissent chez les enfants des Oto comme chez ceux des Pygmées. Les pêcheurs vendent presque tout leur poisson et n'en envoient presque plus au village. Le gibier se raréfie suite à la pression démographique qui oblige aussi à cultiver de plus en plus loin des villages. On remarquera aussi une conséquence du remplacement des commerçants portugais par des commerçants libanais. Les premiers avaient souvent, en plus de leur commerce en ville avec leur famille portugaise, une plantation dans les villages, avec une femme congolaise et avaient des relations étroites et nombreuses avec les villageois, entretenant les routes, réparant les camions, intervenant activement dans les transports. Les Libanais vivent essentiellement en ville (Pagezy H., 1995).

Si les bières restent le véhicule de toutes les ritualisations sociales et la marque quotidienne des liens sociaux et du rapport à l'argent, la production de la bière de banane est devenue une des sources principales de revenus monétaires dans le monde rural rwandais. Sur une colline où la bière de sorgho reste relativement plus importante que la bière de banane, l'expansion récente de cette dernière a été sensible: premier «cabaret» en 1990, quinze en une dizaine d'années. La bière de sorgho garde son rôle social traditionnel. Elle est consommée surtout lors des 
fêtes. Sa production est l'affaire de la femme. Celle de la bière de banane est l'apanage des hommes. Le développement de sa production a été ici indirectement lié à l'implantation d'un projet de développement qui a apporté des ressources monétaires à la colline. II en a été de même pour la consommation de la bière en bouteille (de Lame D., 1995). Sur la consommation des boissons alcoolisées en Afrique, on consultera le livre d'A. Huetz de Lemps qui comporte un chapitre sur les vins de palme, un souschapitre sur la bière de banane dans les Hautes Terres et un sous-chapitre sur la puissance de l'industrie de la bière en R.D.C.(Huetz de Lemps A., 2001).

Les famines et les disettes étaient fréquentes avant la période coloniale et même pendant celle-ci. Chez les Luunda du sud du Kwango, c'était le cas dans la région des Mabeete («vallées» en contrebas du haut plateau près de Panzi, groupement Nzofu), où l'alimentation repose sur le manioc qui connaît des crises répétées et où, faute de routes convenables, les habitants ne peuvent se procurer des ressources monétaires par la vente de leurs produits (sinon la vente des chenilles pour Kinshasa). L'article comporte différents récits de famines et en fait l'analyse anthropologique. Lors des famines, il n'était pas rare d'échanger des enfants contre de la nourriture. On comptait sur les rites pratiqués par le chef luunda «pour faire cesser la faim» (De Boeck F., 1995).

Le Rwanda a connu de nombreuses famines. Celle dite Rwakayikura (19271930) (sa dénomination ferait allusion au chef tutsi dont la chefferie en aurait été le noyau initial) est la première où il y a eu interaction entre la population africaine et un encadrement européen. Elle est survenue après plusieurs mauvaises saisons agricoles, à une époque où l'Est du pays était encore mal relié au réseau commercial et s'est donc trouvé isolé. II y a eu au moins 30000 morts. Plus de 100000 personnes ont émigré. Beaucoup d'hommes ont quitté temporairement les rugos pour se procurer des vivres sur des marchés éloignés. On a consommé de nombreux produits de substitution. La mortalité a été, semble-il, plus forte chez les hommes que chez les femmes (qui tenaient la marmite). Des solidarités traditionnelles se sont dissoutes. Les Européens ont été très sensibles à cette crise mais celle-ci a donné à l'administration coloniale l'occasion d'accélérer sa pression et de mieux contrôler l'espace territorial. Elle a introduit de nouvelles cultures dont le manioc ce qui ne s'est pas fait sans de fortes résistances. Elle a aussi, par la suite, développé le réseau routier, notamment pour favoriser les échanges de nourriture. De même le rôle des missionnaires s'est renforcé. Leurs rencontres plus nombreuses avec la population ont entraîné de nombreuses conversions. En échange de la fourniture de vivres, ils ont pu faire effectuer de nombreux travaux (Cornet A., 1995).

Dans la collectivité de Kafuba (Kasai occidental, Territoire de Kazumba, à 130 $\mathrm{km}$ de Kananga), les menaces de difficultés alimentaires sont fréquentes malgré une production importante de maïs. C'est qu'une part importante de celui-ci est transformée en alcool pour assurer de meilleures rentrées d'argent (Bidinkama Kupa Ngandu et Bilowa Bidiku, 1993).

Toutes ces publications concernent des milieux ruraux. Elles montrent que les problèmes alimentaires se rencontrent dans toutes les parties du pays. Une étude macro-économique indique que la proportion de la population congolaise sous-alimentée a augmenté de $29 \%$ de 1990-92 à 1997-99. Ce serait la plus forte augmentation mondiale pendant cette période. Cette situation est due essentiellement à l'accroissement de la pauvreté et touche donc fortement la population urbaine car la production agricole ne paraît pas avoir diminué. La commercialisation ne paraît pas non plus avoir été défaillante. Le manioc reste la culture de sécurité, notamment suite à la guerre mais il est victime de maladies et de parasites. L'article contient des tableaux de la consommation alimentaire par année de 1961 à 2000, de l'approvisionnement de Kinshasa, des prix des divers produits, de la consommation annuelle à Kinshasa, de 1975 à 2000, et des résultats d'enquêtes nutritionnelles (Tollens E., 2003).

Une étude plus ancienne et dont les 
données sont aujourd'hui certainement très vieillies, affirme que la zaïrianisation avait non seulement diminué la production en décourageant les investissements mais surtout qu'elle avait désorganisé les systèmes d'approvisionnement, de production et de commercialisation. Par rapport à 1982, I'habitant du Congo-Zaïre de 1990 consomme davantage de glucides mais beaucoup moins de lipides. La consommation de protides s'est légèrement améliorée surtout grâce à l'accroissement de la consommation de viande et de volaille importées. La consommation des légumineuses a fortement diminué (Muzigwa Kashema, 1993).

La hausse des prix des produits alimentaires en 2008 s'est fait sentir dans l'alimentation des ménages congolais. En deux mois (de mai à juillet), le coût du panier de la ménagère a augmenté de $42 \%$. L'indice des prix alimentaires est passé de 288 en juin 2007 à 366 en juin 2008. Si l'on exprime la vulnérabilité alimentaire en nombre de repas par jour, on constate que les ménages à un seul repas, à 2 repas et à 3 repas, qui faisaient respectivement $6 \%, 79 \%$ et $15 \%$ du total des ménages en 2004 font $19 \%$, $73 \%$ et $8 \%$ en 2008. La part des ménages très vulnérables a donc augmenté de $216 \%$, celle des ménages non vulnérables a diminué de $46 \%$. Pour sécuriser les approvisionnements, les experts de la Banque Mondiale proposent de concentrer les efforts sur une augmentation de la production agricole dans un certain nombre de régions (à haute potentialité agricole, à population rurale importante et avec un bon accès aux marchés) en préconisant des méthodes et des structures qui ressemblent très fortement à celles des paysannats pendant la colonisation (Tshingombe Mulubay F., 2008).

L'accroissement de la pauvreté des habitants des campagnes est manifeste. Un article général l'étudie pour l'ensemble de l'Afrique sudsaharienne en prenant quelques exemples en Afrique centrale (effets des prélèvements de l'Etat sur les ressources des paysans au Burundi, effets du déclin des transports terrestres dans le Kasai occidental, effets de la diminution relative de la population masculine des campagnes, etc.) (Mwanza $\mathrm{H}$. et Kabamba K., 2002).

Pour accroître la consommation en protéines des populations africaines, surtout dans les grandes villes, les autorités coloniales ont voulu, après la deuxième guerre mondiale, développer la pêche maritime. A la suite de plusieurs expéditions, notamment celle d'A. Capart, on créa en 1950 les Pêcheries Maritimes au Congo. Elles connurent un certain succès mais se dégradèrent fortement après 1974. La zaïrianisation les a dépecées. La flotte disparut aussi presque complètement par suite de naufrages ou d'arraisonnements par les Angolais. L'usine construite pour traiter les poissons existait encore vers 1990 mais les installations frigorifiques étaient en panne depuis longtemps (Lederer A., 1992).

\section{LE SECTEUR INFORMEL}

\section{GÉNÉRALITÉS}

L'économie informelle est une composante essentielle de l'économie nationale. A partir des années 80, le secteur informel aurait acquis au Zaïre un poids équivalent à celui du secteur formel. L'ouvrage qui fournit cette information à partir de l'évolution du PIB, reconnaît trois segments dans ce secteur: le capitalisme sauvage, le segment du petit artisanat et des services, les activités de survie. Dans le segment du «capitalisme sauvage», il constate que l'informel existe aussi au niveau de l'Etat puisque, en 1994, des ministres ont laissé imprimer l'équivalent de plusieurs dizaines de millions de dollars de fausse monnaie. Une grande partie des exportations de diamant a suivi des voies informelles (contrebande) pour un montant équivalant à la totalité des dépenses de l'Etat en 1995. Le même ouvrage étudie l'évolution du secteur du petit artisanat et des services depuis 1946 et, à partir des 
résultats d'une enquête menée à Kinshasa, décrit les stratégies de survie des citadins. Des indicateurs sanitaires montrent l'accroissement de la pauvreté pendant la période dite de transition. La malnutrition globale a augmenté de 1991 à 1995 avec des pics saisonniers, au mois de mars particulièrement, avant les récoltes de la dernière partie de la saison des pluies et quand l'acheminement des produits vivriers par la route est le plus difficile. L'ouvrage cherche aussi à estimer l'efficacité des stratégies d'intervention qui ont été utilisées pour lutter contre la pauvreté, notamment pour la population la plus touchée par les mesures du plan d'assainissement structurel. Le dernier chapitre (par Lubuimi Mukaya Lalaby) décrit le rôle du secteur informel dans la gestion des déchets (De Herdt T. et Marysse S., 1996).

Voici quelques considérations générales macro-économiques sur le secteur informel à partir du portrait de l'homo economicus kinois et de ses relations avec les groupes dont il a besoin pour assurer sa survivance et avec lesquels il doit partager ses revenus. L'économie informelle est une force cohérente. Elle comporte un mélange de marchés concurrentiels et de redistribution des terres, de capitaux, etc. II serait dangereux de formuler des politiques économiques et sociales qui l'ignoreraient totalement (Leclercq H., 1993)

Un colloque, organisé conjointement, en décembre 1994, par l'Association belge des Africanistes et l'Institut Africain, a été consacré aux relations entre les phénomènes informels et les dynamiques culturelles en Afrique (de Villers G., 1996). Trois thèmes: 1) le droit foncier et les pratiques foncières, 2) les identités et les changements culturels, 3) la linguistique et la création langagière. Plusieurs communications concernant spécifiquement le Congo sont citées dans d'autres paragraphes de cette chronique. G. de Villers, dans un bref article, s'interroge sur la capacité que pourrait avoir le secteur informel à aider à la recomposition des systèmes politiques et sociaux, à la restructuration d'un "ordre» social qui serait le résultat d'une synthèse entre des formes d'origine africaine et des formes apportées par la mondialisation (de Villers G., 2000). MacGaffey J. (1995) avait, elle aussi, insisté sur l'instabilité des entreprises du secteur informel, même celles d'une certaine dimension, doutant qu'elles puissent apporter une solution satisfaisante face à l'insécurité permanente. Sur un thème voisin, mais en relation avec les pratiques politiques, voir Peemans J.-P. (1997).

C'est dans les villes que le secteur informel prend les formes les plus diverses. Un livre sur "l'économie de la débrouille» en donne de nombreux exemples à Lubumbashi, Mbuji Mayi, Kisangani et Kinshasa à partir d'enquêtes menées par des chercheurs congolais comportant des interviews et des récits de vie recueillis de 1995 à 1997 (de Villers G., Jewsiewicki B. et Monnier L., 2002). Ces cas concernent le plus souvent des personnes qui ont une occupation officielle (dans l'enseignement, l'administration, l'université, le milieu hospitalier, etc.) mais qui, faute de toucher leur salaire, donc victimes de ce qu'on a appelé la «désalarisation», sont contraintes d'exercer une autre activité dans la sphère informelle. A Kisangani, par exemple, où, à la fin des années 90, les taxis ont pratiquement disparu (et où même les camions se font de plus en plus rares), les transports de personnes se font souvent en toleka. Le toleka est un vélo transportant un client sur son porte-bagage. Le plus souvent il n'appartient pas au conducteur (le porteur) mais est loué à son propriétaire qui en tire ainsi quelques ressources (Omasombo Tshianda J., 2002). Un autre cas significatif est celui d'infirmières de l'hôpital universitaire de Kinshasa, qui, pour survivre, sont contraintes de vendre, aux mères de la maternité, des pulls qu'elles tricotent, des confitures ou des beignets qu'elles fabriquent, des produits cosmétiques ou des bijoux de pacotille que leur envoient des parents d'Europe, etc. (Ndaywel è Nziem I., 2002). Un chapitre traite le cas des cambistes, partie visible du marché des devises, dont les centres se sont déplacés dans la ville de Kinshasa: au départ le beach Ngobila (d'où partent les navettes pour Brazzaville) puis un quartier de Gombe qui fut surnommé Wall Street, 
puis éparpillement en dehors de cette zone, notamment à Matonge (De Herd Y. et Marysse S., 2002). Une communication traite aussi de la paupérisation des familles petites-bourgeoises après 1991, c'est-à-dire à partir de la crise qui a accompagné la «transition démocratique». Auparavant ces familles vivaient décemment grâce à des salaires certes très médiocres mais qui étaient payés (Falangani Myondo Pashi, 2002).

La situation des jeunes médecins issus de l'Université de Lubumbashi en 1997 et 1998 est par certains côtés plus difficile encore car ils considèrent que l'image de leur profession ne leur permet pas d'inscrire leurs stratégies économiques dans une logique de la «débrouille». Ils persistent dans une logique de dépendance à l'égard du seul travail salarié tout en espérant qu'un jour la situation s'améliorera et, objectif idéal, qu'ils pourront créer une clinique privée (il y en a aujourd'hui plus de deux cents dans la ville). Certains émigrent vers l'Afrique du Sud après souvent une sorte de stage en Zambie (Rubbers B., 2003).

\section{LE DÉVELOPPEMENT DE L'INFORMEL DANS L'EXPLOITATION MINIĖRE}

Une certaine libéralisation de l'exploitation minière, à la fin de l'ère mobutiste, et la crise, qui a frappé les grandes entreprises minières du Katanga, ont entraîné la prolifération de petites exploitations artisanales parfois clandestines. C'est dans le domaine du diamant surtout que celle-ci a pris le plus d'ampleur. De nombreux ex-salariés paupérisés par l'inflation ou l'arrêt ou les retards de paiement (employés, fonctionnaires, enseignants) ont pu, dans cette activité, «produire» des dollars. Comme une partie des gisements chevauche la frontière angolaise et que le diamant en Angola a été, à plusieurs reprises la grande ressource financière de la rébellion, on a là un monde particulièrement violent où cette activité participe aussi à une intense contrebande. Plusieurs articles de F. De Boeck décrivent le monde de ces garimpeiros à la frontière du Kwango et du Lunda Norte. Leur activité a contribué à une "dollarisa- tion» de l'économie de ces régions frontalières, dollarisation qui s'est étendue à une bonne partie de l'espace congolais. Des localités comme Kahemba et Tembo ont connu un boom remarquable. Kahemba est passé ainsi de 10000 habitants environ en 1984 à plus de 100000 dix ans plus tard pour retomber fortement après 1998. La recherche du diamant est assimilée à une opération de chasse, de capture, de domptage des diamants et des dollars. On y retrouve donc les rites des chasseurs. L'argent obtenu de cette façon par les Bana Lunda, ces jeunes gens, souvent des diplômés sans emploi, qui partent dans les régions frontalières, est consommé d'une façon généralement ostentatoire dans les villes comme Kikwit pour la bière, les filles, les appareils électroniques (De Boeck F., 2001). Un article traite de la répartition des rôles respectifs des hommes et des femmes dans cette chasse au diamant. Les femmes ne "creusent» pas ou ne plongent pas pour récolter les diamants mais elles fournissent des services aux jeunes gens: repas, commerces divers, services sexuels et parfois aussi participation à l'écoulement des diamants, du moins à un niveau élémentaire, le commerce à un niveau plus élevé étant entre les mains soit de personnages politiques ou militaires soit souvent d'étrangers comme les Libanais (De Boeck F., 1999, 2001).

Un article écrit par des géographes étudie les effets sur la géographie régionale des activités liées au diamant qui se sont développées dans cette zone frontalière. Celle-ci était une sorte de bout du monde pendant l'époque coloniale. Peu peuplée, dotée de sols généralement très médiocres, sur de hauts plateaux sableux, éloignée des centres actifs (Kinshasa et pays huilier du Kwilu), elle connaissait fréquemment de graves problèmes alimentaires. Depuis le début des années 80, l'exploitation artisanale de gisements alluviaux de diamant dans la vallée du Kwango, en territoire congolais, et la participation de jeunes Congolais à l'exploitation des mines du Lunda, ont entraîné un afflux de population et même donné naissance à de nouvelles agglomérations comme Tembo (près des 
chutes du Kwango) et accru fortement l'importance de l'ancien poste administratif de Kahemba qui est devenu une sorte de Far West. II n'y eut guère de transformation de la production agricole mais les flux à travers la frontière, souvent à caractère clandestin, se sont développés. Ils ont permis, pendant tout un temps, à des régions angolaises rebelles au gouvernement national, de recevoir par la route, du Congo-Zaïre, carburant, aliments, produits manufacturés et armements, qui ne pouvaient plus lui parvenir directement de la côte. Mais toutes ces situations sont précaires et fluctuent en fonction des aléas des guerres civiles. L'exploitation du diamant n'apparaît guère comme un ferment régional de développement ou d'unité (Maboloko Ngulambangu et Nicolaï H., 1999).

Un volume des Cahiers africains a repris la métaphore relative à la chasse comme titre d'un ouvrage collectif consacré à l'activité diamantaire (Monnier L., Jewsiewicki B. et de Villers G., 2001). On y lira notamment une présentation des Bana Lunda, avec plusieurs récits de vie particulièrement éclairants, par Sabakinu Kivilu et une communication sur l'apparition de seigneurs du diamant à Kisangani, par Omasombo Tshonda. Le cas de Kisangani est très significatif car le diamant n'y est exploité que depuis la découverte de quelques gemmes en 1986 à une quarantaine de kilomètres sur la route de Buta. Auparavant les sociétés minières considéraient que les ressources étaient beaucoup trop faibles pour permettre leur exploitation. Kisangani a connu une véritable fièvre du diamant. La communication se fonde sur une enquête menée par le prof. Bilusa (Université de Kisangani) de février 1993 réactualisée par l'auteur en 1996-97, dans une trentaine de carrières de la forêt où près de 20000 personnes vivent dans des villages de «creuseurs», commerçants, acheteurs. L'exploitation artisanale de l'or et du diamant et plus particulièrement ses répercussions sur l'environnement ont été étudiées dans l'ancienne Province Orientale (Haut-Zaïre). Notons qu'ici aussi, comme dans le cas des plongeurs du KwangoLunda, les accidents mortels qui frappent parfois les «creuseurs", lors de l'effondre- ment d'un puits par exemple, sont perçus souvent comme des sacrifices quasi nécessaires par lesquels on s'attache la bienveillance des esprits du lieu et qui garantissent la fécondité du gisement (Indring'ia N. et Bahuminga I.B., 1998). Cette exploitation a connu une forte accélération à partir de 1990 quand le processus de "démocratisation» a fait croire que toute forme de gouvernement hiérarchique relevait du défunt parti unique et n'avait donc plus de raison d'être. Ici aussi l'article décrit un certain nombre de personnages caractéristiques: le propriétaire ou PDG qui a découvert le gisement, l'ayant droit, c'est-à-dire le chef de terre à qui l'on verse des royalties, le fournisseur, homme d'affaires citadin ou riche commerçant qui fournit les capitaux et le matériel, le chef de camp, souvent déserteur de l'armée ou repris de justice qui surveille les travailleurs, les boulonneurs, manoeuvres des puits, le brigadier, agent de l'ordre recourant aux châtiments corporels, le trafiquant, en contact avec les acheteurs qui parcourent le pays.

Plusieurs publications traitent de l'utilisation légale ou illicite des revenus issus des diamants par les hommes politiques au pouvoir et les mouvements rebelles ainsi que des rivalités entre différents pays africains pour les contrôler. Le terme «gemmocratie» est utilisé pour qualifier les régimes qui recourent à cette ressource (Misser F. et Vallée O., 1997). Un séminaire a été consacré à cette question par l'Association Belge des Africanistes. On trouve aussi, dans le livre sur «La chasse au diamant», des mêmes auteurs, un article qui montre comment la Miba, qui produit les diamants de Mbuji Mayi et qui était parvenue à maintenir son activité grâce notamment à sa gestion par une société belge, actionnaire minoritaire, liée au groupe De Beers, a été pillée par Mobutu et ses ministres puis par ceux de Laurent Kabila (Misser F. et Vallée O., 2001). Un autre article évalue l'importance du diamant (formel et informel) dans l'économie congolaise (Leclercq $H$., 2001).

Pour le petit secteur informel et les activités de survie, on consultera plus loin le chapitre consacré aux villes. 


\section{L'ÉCONOMIE NATIONALE FORMELLE}

\section{L'ÉVOLUTION ÉCONOMIQUE}

Il va de soi que l'évolution économique du Congo a été traitée dans de nombreux articles de périodiques financiers ainsi que dans des rapports émanant des ministères concernés, dans les rapports annuels de la Banque du Zaïre-Congo et dans la revue «Notes de conjoncture». Nous ne citons ici que quelques exemples d'articles publiés dans des revues de sciences humaines. Des chroniques régulières de la revue Zaïre (Congo)-Afrique fournissent le texte ou des extraits, et souvent des commentaires, des multiples programmes gouvernementaux successifs de stabilisation et de relance de l'économie. Un article publié en 1993, par exemple, sur la politique économique zaïroise et ses perspectives n'a évidemment plus beaucoup de valeur en ce qui concerne les perspectives qui y étaient tracées mais il garde de l'intérêt pour sa synthèse de l'évolution économique du pays avant 1993. Le taux d'accroissement du PIB est devenu négatif depuis 1989 avec, chaque année, une valeur absolue de plus en plus forte (Muhinduka Di-Kuruba, 1993). Le numéro 321 de la revue (janvier 1998), expose le Programme de stabilisation et de relance de l'économie congolaise, communication du Gouvernement à la réunion des Amis de la République Démocratique du Congo qui s'est tenue a Bruxelles en décembre 1997. Dans le numéro 375 (2003), on trouvera des extraits du rapport de la Banque Centrale du Congo sur l'évolution économique du pays de 1990 à 2003. Le PIB, en termes réels, a reculé de $43,7 \%$, la plus forte diminution se produisant en début de période, soit 10,8 \% par an entre 1990 et 1993. Un chiffre montre le déclin de l'économie formelle: le nombre de salariés a diminué de $85 \%$ de 1990 à 2000, passant de $12,3 \%$ de la population active à 3,1\% (Institut National de la Statistique, 2003). Le numéro 383 (2004) «Les problèmes actuels...») comporte des articles de hauts fonctionnaires congolais ou internationaux sur les problèmes de l'économie, notamment un exposé du programme écono- mique du gouvernement (Masangu Mulambo, 2004), un bilan du programme intérimaire renforcé (Ngonga Nzinga, 2004), l'intervention du Fonds Monétaire International (de Kouwenaar A., 2004). Les dernières années du régime mobutiste ont été tumultueuses du point de vue économique.

\section{LA RÉFORME MONÉTAIRE}

La réforme monétaire de 1993 a créé une nouvelle unité de compte, le nouveau zaïre (NZ) qui s'est échangé contre 2 millions de zaïres anciens, 3 NZ contre 1 dollar américain. Elle a été accompagnée de manoeuvres douteuses, équivalant pratiquement à la fabrication par l'Etat luimême de fausse-monnaie quand la confection des billets a été confiée à des commerçants libanais qui ont été rémunérés par un prélèvement sur les billets qu'ils émettaient et qui, semble-il, ont imprimé cinq à six fois les mêmes séries (à l'insu ou non des responsables politiques?). Ces incidents et toutes les tentatives de réformes, dans cette période agitée, sont décrits dans le livre de Mutamba Lukusa (1999). Une autre expression du désordre monétaire, vrai cas d'école pour les économistes, a été, dans certaines parties du territoire, le refus de la monnaie émise par l'Etat central. Le nouveau zaïre de 1993 a été refusé par les habitants des deux Kasai qui ont continué à utiliser et à n'accepter que les anciens zaïres, circulant d'ailleurs en même temps que le dollar. Cela s'est produit dans une province qui avait une ressource locale forte (le diamant de Mbuji Mayi) qui lui a permis de soutenir l'ancienne monnaie. Ce refus de confiance dans la monnaie nationale a peut-être aussi été renforcé par l'écartement au plan national d'un leader politique local et aussi par le délabrement des moyens de communication qui a assuré un isolement relatif par rapport à la capitale. Cet espace monétaire kasaïen, qui se situait à la fois dans et hors de la zone monétaire nationale, en somme une révolte monétai- 
re, s'est maintenu jusqu'en 1998 alors qu'on s'attendait à ce qu'il disparût après un an par suite de l'usure de billets qui ne pouvaient être remplacés. Son histoire est décrite avec talent dans un livre qui la replace aussi dans le cadre de la théorie de la monnaie. II n'est pas absurde d'envisager que de nouveaux espaces monétaires de type kasaïen seraient possibles encore au Congo en cas de reproduction des mêmes conditions économiques et politiques (Kabuya Kalala et Matata Ponyo Mapon, 1999).

Un autre événement économique a été la dollarisation d'une partie de l'économie pour faire face à l'hyperinflation. Cette dollarisation est traitée succinctement dans le livre dont il vient d'être rendu compte. Une des sources initiales de dollars a été l'exploitation artisanale (parfois clandestine) du diamant et de l'or et leur trafic dans les régions frontalières dont nous avons déjà parlé. Voir aussi Ngonga Nzinga (1999).

\section{LE PILLAGE DES RESSOURCES}

Les rébellions armées et les troupes étrangères, qui ont occupé une partie du pays pendant la guerre de 1998, sont accusées d'avoir organisé un pillage des ressources. Qu'en a-t-il été à Kisangani? Le monopole d'achat de la production locale de diamant a été attribuée par les occupants rwandais à un commerçant libanais de la ville. Une partie des ressources locales a été dirigée vers I'Uganda. Plusieurs machines de l'usine textile auraient été transférées à Kigali. Les taxes locales (par exemple sur les cyclistes-taximen) auraient été confisquées par les occupants. Pendant la guerre, la principale source de revenus monétaires pour les habitants de Kisangani a été constituée par les prestations fournies aux fonctionnaires de la MONUC. La mortalité générale aurait été doublée par rapport aux années d'avant la guerre et la mortalité infantile multipliée par quatre (Kabamda P., 2004).

\section{L'APPARITION DU TÉLÉPHONE MOBILE}

Celle-ci a été un événement technique essentiel pour l'Afrique centrale au cours de la dernière décennie. Le téléphone mobile a bouleversé les relations entre les diverses parties du pays mais aussi à l'intérieur des grandes villes, les systèmes antérieurs (téléphones fixes et radio-téléphones) étant peu fiables ou très encombrants. On pourrait dire que le Congo a accédé directement à l'âge du téléphone mobile sans être passé par le stade du téléphone fixe. Aucune étude ne semble avoir encore été faite jusqu'à présent sur ce sujet. Par contre voici un article sur un service lié à la diaspora et qui est de plus en plus visible dans la ville: l'agence de la société de transfert de fonds par où transite une grande partie des fonds envoyés d'Europe (Bagalwa-Mafatano J. et Monnier L., 2002).

\section{LA MOBILITÉ ET LES TRANSPORTS}

Ce qu'on appelle traditionnellement la Voie nationale, et dont le tronçon principal est la liaison mi-ferroviaire mi-fluviale du Katanga au port de Matadi, était en 1993 (et est encore aujourd'hui), dans un état de forte détérioration. Les structures fluviales étaient menacées tant sur le Kasai (le quai d'llebo) que dans les passes mal entretenues du secteur maritime. Les communications étaient meilleures par le rail Tanzara vers Dar es Salaam auquel était promise une importance de plus en plus grande étant donné la part croissante prise par les clients asiatiques des produits katangais (Charlier J., 1993).

La dégradation de tous les modes et systèmes de transport hérités de la colonisation belge a paralysé progressivement les échanges dans tout le Congo. Un article en fait une analyse générale. La rupture des encadrements après 1960 a été un facteur essentiel. Une bonne partie de la qualité du réseau routier, pendant la période coloniale, était en effet la conséquence d'encadrements contraignants pour la population et notamment pour la population des campagnes. La zaîrianisation des entreprises de transports de toute taille a aggravé encore la situation. La dégradation des transports a handicapé le développement de l'agriculture tout particulièrement de l'agriculture vivrière destinée aux agglomérations urbaines. Cela 
vaut aussi pour les tentatives de relance de l'activité cotonnière dans le nord-est du pays. La société textile de Kisangani a été contrainte par exemple d'usiner du coton américain dans une proportion de $50 \%$. L'état des routes est trop aléatoire pour permettre la mise en place de chaînes de froid avec des camions frigorifiques (pour le ravitaillement en viande des grandes agglomérations par exemple à partir de régions éloignées comme l'Ituri). Un système qui a donné quelques résultats: la concession par l'administration centrale de l'entretien d'une route à une entreprise (société agroindustrielle) ou une institution (religieuse comme un diocèse par exemple) mais le tableau d'ensemble reste sombre. Des régions, dont l'isolement croît, apparaîtraient condamnées à la régression (Pourtier R., 1993).

Un gros mémoire étudie la problématique de l'intégration économique par les transports. II rappelle l'effondrement progressif de l'organisation des transports héritée de l'époque coloniale et l'émergence des acteurs privés depuis 1960. II insiste sur le dynamisme de ceux-ci dans la reconstruction du système, par exemple dans la création de nouveaux itinéraires comme ceux qui relient Kikwit aux centres miniers de la frontière angolaise. II analyse le rôle de certaines ONG comme le CDIBwamanda et le Développement Progrès Populaire Idiofa dont il a été question plus haut. Aucun investissement nouveau dans les transports n'aurait été effectué de 1990 à 1997 ni de la part de l'Etat ni de sources étrangères. Le gouvernement Kabila aurait remis en question la politique de privatisation et de restructuration des gouvernements de transition, le Sizarail, par exemple, redevenant la Société Nationale des Chemins de Fer du Congo (SNCC) (M'Pene Ngaluley Z., 2002).

La débâcle ferroviaire en RDC, manifeste dans le cas emblématique du rail MatadiKinshasa (Charlier J. et M'pene Z., 2006) est replacée dans le cadre général de l'avenir du rail en Afrique par R. Pourtier. Pour cet auteur, malgré la présentation périodique de projets utopiques, le rail ne pourra se maintenir que dans le transport des produits miniers (ce qui ne permet pas la constitution de réseaux) ou peut-être dans la desserte périphérique des grandes agglomérations (Pourtier R., 2007).

Une étude topologique du réseau routier congolais aboutit à des conclusions peu surprenantes. La connectivité est faible et mauvaise tant pour l'ensemble du pays que pour chaque province. II en est de même de l'accessibilité. Les résultats sont très théoriques car ils ne tiennent pas compte de l'état des voies (Bukome Itongwa D. et Kingoma Munganga J.-P., 2002).

Le désenclavement du Rwanda et du Burundi se fait principalement par la liaison avec l'Océan indien (ports de Mombasa et de Dar es-Salaam). Au départ du Rwanda, celle-ci utilise la route puis le rail en Uganda. Au départ du Burundi, le lac Tanganyika puis à Kigoma, le rail à travers la Tanzanie. Mais une part de plus en plus importante du trafic se fait par acheminement routier direct. Cependant de nouvelles formules intermodales s'appuyant sur des terminaux intérieurs en Tanzanie et en Uganda ont permis au chemin de fer de regagner du terrain (Charlier J., 1997).

Le lac Kivu est une voie de transport commode. Une flotte de pirogues assure une partie du ravitaillement vivrier des agglomérations de Bukavu et de Goma et même de l'île très fortement peuplée d'Idjwi (Gakinahe G., 1993).

Revoici l'idée d'une liaison fluviale entre l'Atlantique et Kinshasa contournant les rapides et les chutes. Des études menées depuis 1972 à l'Université de Liège concluent à la fiabilité d'un canal de navigation à grand gabarit (péniches de 6000 tonnes). Le projet comporterait un canal entre l'amont d'Inga et la région de Boma, avec une grande écluse ou un ascenseur funiculaire. A $200 \mathrm{~km}$ en amont d'Inga, un barrage en pierres lancées ajouterait une puissance d'au moins 15000 MW aux 39000 MW disponibles d'Inga. Ces travaux évidemment colossaux représenteraient une dépense (50 milliards d'euros dont 3 milliards pour le canal et les écluses) du même ordre de grandeur que celle de l'aménagement des Trois Gorges sur le Yang-Tsé (Arnould R., 2005). 


\section{LE PROBLÈME DE L'ENSEIGNEMENT UNIVERSITAIRE}

Un article rappelle la brève existence de I'Université protestante du Congo (Université Libre du Congo). Née peu après l'indépendance du Congo (la presse belge la qualifia, à l'époque, d'université «américaine»), elle s'installa de façon éphémère à Stanleyville en 1963 puis fut accueillie à Lovanium en 1964 avant de se limiter finalement à une Faculté de théologie protestante installée sur le campus de Kinshasa (Deelstra H., 2001).

R. Devisch peint un tableau désolant de la dégradation de l'université de Kinshasa depuis l'époque de Lovanium. Elle se trouve depuis des années sans budget de fonctionnement et sans aides substantielles. Fleuron de l'activisme catholique belge des années cinquante-soixante, elle s'écroula après sa nationalisation en 1971 puis s'effondra davantage encore après 1990. Certains professeurs, qui se sont opposés au pouvoir en place, se sont trouvés clochardisés. R. Devisch s'interroge sur le modèle que cette université a représenté et plaide pour une «endogénisation» des universités africaines du moins en sciences humaines, concept multidimensionnel assez flou qu'il serait trop long de développer ici (Devisch R., 1999).

Au cours des dix dernières années, les intellectuels congolais et particulièrement les professeurs des universités se sont demandé comment ils pourraient contribuer à rétablir la démocratie, la paix et la bonne gouvernance dans leur pays. En septembre 1999, l'Association des Professeurs de l'Université de Kinshasa, présidée par Sabakinu Kivilu, association qui a joué un grand rôle dans le maintien d'un fonctionnement acceptable de l'Université, a organisé un colloque sur «Démocratie et paix en RDC» (Sabakinu Kivilu, 1999). Dans la partie consacrée aux expériences historiques dans ce domaine au Congo, on retiendra une communication de Makwala dans laquelle il s'efforce de montrer que la société kongo traditionnelle comportait des instruments qui assuraient la paix et un certain type de fonctionnement démocratique. Diverses pratiques favorisaient la participation de ses membres à l'exercice du pouvoir, protégeaient leurs droits, les associaient au choix de leurs dirigeants, limitaient le pouvoir des chefs, assuraient l'instruction des jeunes gens destinés à devenir des chefs. Des mécanismes sauvegardaient la paix, comme le recours à la palabre et l'appartenance à des clans qui étaient représentés dans tout le pays kongo (Makwala, 1999). Une communication de Sabakinu Kivilu montre que si la Conférence nationale souveraine n'a pas abouti à son projet de grande réconciliation nationale, elle a été un facteur de mobilisation politique et sociale et a éveillé les consciences politiques (Sabakinu Kivilu, 1999). L. de Saint Moulin analyse quelques-uns des obstacles qui s'opposent à l'avènement de la démocratie en se fondant sur différentes enquêtes qu'il a menées avec ses étudiants dans différentes communes de Kinshasa sur la perception de la démocratie et des élections, sur celle de la justice et du mal. Plusieurs de ces enquêtes ont été citées plus haut dans la présente chronique (de Saint Moulin L., 1999). Parmi les nombreux thèmes abordés dans le même ouvrage, on notera une recherche sur le rôle que la femme peut jouer dans une culture de paix, une communication sur la question de la nationalité (par Kapeta Nzovu), une réflexion sur les dérives identitaires qui montre par exemple les identifications erronées et précipitées fondées sur le nom ou sur la langue parlée (par Obotela Rachidi N.). Une quatrième partie étudie les rapports entre les conditionnalités démocratiques et les exigences politiques et économiques. La cinquième partie traite des jeux et des enjeux de la paix en Afrique centrale face aux exigences internationales, avec notamment un article de Ndaywel è Nziem qui invite à réfléchir sur la notion d'Afrique centrale et sur le danger de croire que, par ses ressources naturelles, le Congo est un pays prédestiné à jouer un rôle précis dans le monde africain. Une communication de Ntumba Luaba sur la géopolitique des grandes 
puissances a été présentée plus haut dans cette chronique (p.306). Une autre publication dirigée par Sabakinu Kivilu s'interroge sur le rôle que doit jouer l'université congolaise dans le rétablissement de la paix et la construction de la démocratie. On y trouve, une communication de cet auteur sur l'émergence "d'une nouvelle conscience politique et sociale au sein du corps professoral de I'Université de Kinshasa» (Sabakinu Kivilu I., 2000). Les mêmes préoccupations apparaissent dans un colloque organisé à Bujumbura en 1998 (Gahama J., 2001).

Dans la foulée des mêmes préoccupations et plus précisément dans la recherche d'un projet de société, des séminaires ont été organisés sur la moralisation de la vie publique et sur les nouvelles institutions à mettre en place. Un Institut pour la Démocratie et le leadership politique (I.D.L.L.P.) créé par des professeurs des universités de Kinshasa et du Bas-Congo, dans le but de contribuer, par le travail scientifique et la vulgarisation, à l'émergence d'institutions démocratiques au Congo, a ainsi mis sur pied en 1999 et 2000 cinq séminaires et publié quatre fascicules sur le rôle de la police dans la reconstruction nationale, sur la justice et la société, sur les finances et la démocratie, sur l'administration et le développement. Chaque fascicule se termine par un rapport et parfois par des recommandations. Comme pour l'ouvrage de l'Association des professeurs de l'université de Kinshasa cité plus haut, ces séminaires et ces fascicules ont bénéficié du soutien financier de la Fondation Konrad Adenauer (Mabiala, 1999; Mutunda, 1999; Tshungu, 2000;
Makwala, 2000). De telles publications malheureusement aboutissent rarement dans des bibliothèques d'Europe. II est fort probable qu'il y en ait eu ainsi bien d'autres dont nous n'avons pu avoir connaissance.

Après la répression des étudiants décidée par les autorités publiques en 1990 (qui avait entraîné la rupture de la coopération belge), des fondations canadiennes ont aidé l'Université de Lubumbashi à survivre. Des associations africaines et américaines soutenues par les Fondations Ford et Rockefeller ont lancé un programme sur "Le rôle public des universités en Afrique». Dans le cadre de ce projet, un ouvrage a été publié sur l'histoire de l'Université de Lubumbashi de 1990 à 2002 (Jewsiewicki B. et Klauber V., 2003). L'historique même de I'Université y fait l'objet d'un rapport de Donatien Dibwa dia Mwembu, qui est le coordonnateur du projet à Lubumbashi. On retiendra par exemple que le recteur était parvenu à empêcher l'expulsion des membres du corps enseignant originaires du Kasai. II faut dire que cela aurait privé I'Université de la majorité de son personnel scientifique. Le livre est publié dans une collection soutenue par le CELAT (Canada) et intitulée "Mémoires lieux du savoir. Archives congolaises" dirigée par B. Jewsiewicki. Cette collection, publiée par L'Harmattan, Paris, compte aujourd'hui près d'une vingtaine de volumes. On y trouve de nombreux récits de vie (d'étudiants, de professeurs) mais aussi par exemple, une étude sur les Banyamulenge $(2001,292$ p.) et un aperçu historique «De l'Union Minière à la Gécamines» (2001, 256 p.).

\section{LES VILLES ET LES PROBLÈMES URBAINS}

\section{L'HISTOIRE URBAINE}

J.-L. Vellut a coordonné un numéro des Cahiers Africains où la problématique de I'histoire urbaine en Afrique noire est étudiée de façon pluridisciplinaire. On trouvera ci-dessous le compte rendu de plusieurs des articles qui le composent (Vellut J.-C., 2007)

\section{Les villes précoloniales}

On peut considérer comme des organismes urbains les capitales royales d'Afrique centrale, particulièrement celles des royaumes de la savane, kanyok, ruund (lunda) et luba dans le Kasai oriental et le Katanga. Leurs marques actuelles, visibles sur les photos 
aériennes accompagnant l'article qui les décrit, sont des îlots forestiers englobant les tombes des rois kanyok sur le lieu où se trouvait le sérail du roi. Ces espaces étaient entretenus par un esclave, gardien de la tombe, qui mettait le feu tout autour vers le mois d'avril pour empêcher les grands feux de brousse de la saison sèche de détruire l'îlot. Ces capitales politiques étaient aménagées généralement sur une crête, selon un modèle comportant un plan orienté et une organisation en quartiers. Au centre, le bâtiment prestigieux du roi; en aval, la cuisine pour le roi et ses invités, en amont une place semipublique pour les audiences et, plus en amont encore, la résidence de la reinemère où se trouvait l'entrée publique. Des quartiers homogènes (épouses fécondes, épouses stériles, notables, artisans, guerriers) s'organisaient tout autour, reproduisant l'image du pays. C'est à côté de cette capitale que venaient camper les commerçants étrangers, les hommes fuyant d'autres territoires et où se sont installés, par la suite, les représentants de l'autorité coloniale. II est fâcheux que ce thème très intéressant soit traité de façon fort confuse et que l'auteur, dans sa première ligne, situe le pays des Kanyok à des altitudes andines ou tibétaines (4000 m! sans doute s'agit-il de pieds et non de mètres!) (Ceyssens R., 2007). On trouvera aussi des informations sur la naissance et l'organisation des capitales luba dans les publications de P. Petit signalées plus haut (Petit P., 1996a, 1996b).

Les enclos royaux agro-pastoraux, qui constituaient les capitales de l'ancien Burundi, pouvaient-ils être considérés comme des villes? II est difficile de le soutenir car la population n'y était pas stable, il n'y avait pas d'échanges commerciaux à longue distance, ni de métiers spécialisés contrairement à ce qui existait par exemple au Buganda (Chrétien J.-C., 2004).

\section{L'urbanisme colonial et postcolonial}

Depuis 1960, peu d'études ont été publiées sur l'architecture de la ville coloniale. Depuis que les lieux de mémoire retiennent une attention croissante et que presque toutes les grandes villes congolaises ont atteint ou dépassé leur centième anniversaire, on s'y intéresse davantage. L'architecte John Lagae a consacré à ce sujet une thèse de doctorat à I'Université de Gand, en 2002. Une autre thèse a été soutenue à la KUL en 1994 par Bruno De Meulder sur l'architecture et la planification urbaines à Lubumbashi. Ces thèses sont restées inédites mais plusieurs publications et des expositions en ont repris et parfois développé certaines parties. Voici d'abord un article sur la maison coloniale idéale au Congo de 1885 à 1960. Le débat a concerné longtemps l'adaptation de la maison européenne aux conditions climatiques tropicales, y compris aux menaces de l'environnement sur la santé. On a généralement privilégié un modèle repris des colonies anglaises ou hollandaises, le bungalow. Le plus souvent au milieu d'une grande parcelle, il était pourvu d'une galerie extérieure, la barza. Parfois des bâtiments annexes étaient prévus pour loger la domesticité, comme dans les villes katangaises. Du modèle du bungalow, on passa à celui de la villa. C'est seulement au milieu des années 1950 que l'on a construit, à Léopoldville, les premiers grands immeubles à appartements. La préoccupation psychologique n'était pas négligée. La maison devait donner une sensation de bien-être à l'Européen isolé, puis, Iorsque celui-ci est venu d'Europe avec son épouse, l'objectif était qu'on puisse y vivre "comme chez soi», sousentendu «comme en Europe». Mais dans la carrière coloniale, la maison est toujours restée un lieu de résidence temporaire. On s'est préoccupé aussi de la maison des travailleurs africains qui aurait dû également pouvoir faire face aux menaces de l'environnement tropical mais qui devait être de construction simple, rapide et bon marché et rester compatible avec les habitudes de populations récemment arrivées de la campagne (Lagae J., 2000).

L'Illustration congolaise, une revue publiée entre les deux guerres et qui a repris certains rôles du Mouvement Géographique, comme celui de familiariser les Belges à leur action coloniale et 
susciter des vocations, consacre une grande part de son iconographie à des vues des villes du Congo. II n'est pas étonnant qu'un tiers de celles-ci concernent Elisabethville et Jadotville (beaucoup plus que Léopoldville) qui montrent le mieux l'Afrique moderne et industrielle construite par les Belges (Morimont F., 2001). Voir aussi, sur un sujet proche, mais pour la période 1946-1952, l'analyse de la représentation de la ville coloniale et des valeurs urbaines, dans une collection de photographies d'un organisme public d'information (Hiraux F., 2007).

La conception urbanistique de la capitale du Congo belge se réfère pour de nombreux points à certains quartiers de Bruxelles. Dès les années 1920, des plans ont été dressés pour le quartier où devaient s'installer l'administration générale de la colonie et les résidences des hauts fonctionnaires, c'est-à-dire le quartier de Kalina (actuellement Ngombe) en bordure du fleuve. Le premier modèle a été le quartier bruxellois du Parc où se côtoyaient pouvoir exécutif, direction des milieux d'affaires (y compris les affaires coloniales) et pouvoir législatif. Le Mont des Arts aussi a servi de référence. En tout cas les péripéties de la construction d'une résidence pour le gouverneur général, qui aurait été à la hauteur de sa fonction, sont très instructives. On imagina d'abord quelque chose qui ressemblait au bâtiment du Musée de Tervuren et au Palais Royal de Bruxelles, puis un palais de style mauresque avec une tour minaret, qu'on considérait comme bien adapté à un milieu tropical mais dont l'aspect était en contradiction avec les mythes fondateurs du Congo, tout particulièrement la campagne arabe. Le bâtiment, qui fut enfin construit dans les années 1950, n'était pas achevé en 1960 et fut utilisé comme siège du parlement du nouvel Etat. En fait, les autorités belges de Bruxelles ont toujours été réticentes à édifier une construction qui aurait pu faire croire que le gouverneur général disposait d'un pouvoir et d'une autonomie que l'on n'a jamais voulu lui accorder (Lagae J., 2007).

Autre phénomène de miroir mais en sens inverse cette fois: en 1960, des archi- tectes qui avaient travaillé sur les projets de planification des quartiers africains, en y appliquant certains théories urbanistiques, sont revenus en Belgique, ont constitué un bureau d'études et ont transféré ces théories à plusieurs projets dont ils ont été chargés à Bruxelles, notamment dans le Pentagone (Lagae J., 2008a). Le numéro 38 des Cahiers de la Fonderie, consacré au thème "Bruxelles et le Congo" comporte plusieurs articles sur la présence (ou le souvenir) du Congo dans la ville actuelle.

Que faire du patrimoine bâti colonial? II est commun de prétendre que la ville coloniale congolaise compte rarement des bâtiments de grande valeur architecturale ou de grande originalité. Les bâtiments étaient conçus par des ingénieurs ou des architectes des services techniques de l'administration. II y avait très peu d'architectes indépendants. La Grand-Poste de Kinshasa était à peu près le seul édifice public témoignant de quelque recherche. Elisabethville comportait davantage de bâtiments conçus par des architectes. Tout le centre de la ville européenne, près de la gare, affichait une incontestable homogénéité. Mais aujourd'hui, avec la reprise de l'activité économique, beaucoup de bâtiments sont détruits au profit de bâtiments plus «fonctionnels» ou plus «modernes». Problème de toutes les villes coloniales: faut-il préserver le patrimoine construit par le colonisateur? L'ICOMOS (International Council on Monuments and Sites) a banni l'expression d'héritage construit colonial pour la remplacer par celle d' "héritage construit partagé» (Shared Built Heritage) étant donné notamment qu'un Etat ou un organisme public peut difficilement avoir aujourd'hui comme projet de restaurer une marque du passé colonial. Dans le «patrimoine partagé» de Lubumbashi, il y a incontestablement le théâtre conçu par C. Strebelle, construit au milieu des années 50 avec de grosses subventions de l'Union Minière et du B.C.K. Le théâtre est devenu le siège du parlement de l'Etat du Katanga en 1960, a figuré sur les billets de la Banque Nationale du Katanga et est aujourd'hui le siège de l'Assemblée provinciale. Le centre culturel qui y était annexé est deve- 
nu le Musée national de Lubumbashi. La synagogue, construite en 1929 par l'architecte R. Cloquet, qui fut l'architecte de l'exposition internationale d'Elisabethille de 1931, témoigne d'une présence juive naguère importante et pourrait être considéré comme un autre élément de ce patrimoine ou du moins d'un «lieu de mémoire», ainsi que le cimetière israélite (mais certains ont contesté l'idée que la notion de «lieu de mémoire» conçue par Pierre Nora soit transposable en Afrique ex-coloniale). Le «lieu de mémoire» principal de Lubumbashi n'est-il pas la cheminée de l'Union Minière-Gécamines avec le crassier (aujourd'hui en voie de destruction)? (Lagae J., 2008b).

Sur les traces du passé à Lubumbashi, voir aussi le numéro des Cahiers africains, édité par Danièle de Lame et Donatien Dibwe Dia Mwembu (2005) et une communication, par ce dernier auteur, sur une opération de sensibilisation de la population de Lubumbashi à la mémoire de sa ville et notamment de son passé industriel, à travers plusieurs expositions organisées par le département d'histoire de l'université au musée national. Sur la question des musées en Afrique, où, trop associés à l'époque coloniale, ils seraient boudés par les autochtones, voir le livre édité par Bouttiaux A-M. (2007).

Les minorités urbaines dans la ville européenne. La ville coloniale comportait, dans sa population non-africaine, des communautés ayant conservé leur originalité. On trouvera des informations sur la communauté juive, qui était particulièrement présente dans les villes du Katanga (Juifs sépharades surtout, venus de l'île de Rhodes) dans plusieurs ouvrages (Bourla Errera M., 2000; Rahmani M., 2002; Benatar J. et Pimienta-Benatar M., 2000), ainsi que dans un livre sur la communauté juive le long du rail du Cap au Katanga (Macmillan H. et Shapiro F., 1999). La localisation des autres minorités (portugaise, grecque, italienne) n'était pas non plus sans signification.

Les églises occupent une place importante dans l'architecture urbaine coloniale, tant dans les quartiers européens que dans les quartiers africains. Là aussi, on a voulu parfois «tropicaliser», mais le plus souvent cela consistait à laisser l'air entrer et circuler. On a voulu aussi, mais plus rarement - et plus tardivement - «africaniser» (on disait «indigéniser») mais cela n'a souvent concerné que des détails de la décoration intérieure. II faudra attendre la période postcoloniale pour que de nouvelles recherches stylistiques apparaissent avec des architectes africains. On le voit bien dans le cas des églises catholiques de Kinshasa. Signe des temps: alors que l'église se dressait au milieu d'un espace public ouvert, elle est désormais dans un enclos ceint de hauts murs avec une grille métallique d'entrée (Mabiala Mantumba Ngoma, 2007).

\section{LA CROISSANCE URBAINE ET LES RÉSEAUX URBAINS}

L. de Saint Moulin continue à suivre la croissance des villes du Congo et les structures de son réseau urbain. Une de ses dernières publications sur ce sujet comporte des tableaux donnant la population des villes et cités en 1958, 1970, 1975, 1984 et 2004 et commente la situation de 2004. A cette date, la population urbaine fait $34,5 \%$ de la population totale et compte 25 villes de plus de 100000 habitants, 19 villes de 50000 à 100000 et 35 centres de moins de 50000 habitants (de Saint Moulin, L., 2007).

J.C. Bruneau avait constaté, dix ans plus tôt, que la crise avait ralenti l'accroissement urbain (5,6\% par an de 1970 à 1984 contre $8,7 \%$ de 1958 à 1970), avec une tendance à la stagnation pour Kananga, Matadi, Mbanza Gungu, Boma, Kikwit, Bandundu, Bukavu et même à la régression pour Kasongo et Kisangani. II affirmait aussi avoir constaté des phénomènes d'exurbanisation, c'est-à-dire de retours d'habitants des grandes villes dans les campagnes où la baisse du niveau de vie aurait été moins forte. Le poids des villes moyennes et des petites villes avait augmenté. Mbuji Mayi, Kolwezi, Tshikapa, Uvira et Butembo, pour des raisons quelque peu différentes d'une ville à l'autre, avaient cependant maintenu leur croissance. Malgré une 
certaine décélération, Kinshasa avait renforcé sa primauté (Bruneau J.-Cl., 1995). Mais les troubles de la fin du siècle ont provoqué une reprise de l'exode vers les grandes villes, postérieure à la publication de cet article. Nous n'avons pas de données précises sur l'ampleur de cette reprise ni sur son évolution.

Nous avions quelque peu actualisé, au même moment, un article antérieur sur les réseaux urbains en Afrique tropicale (Nicolaï H., 1995) et utilisé, dans une comparaison de la ville en Afrique et de la ville en Europe, quelques cas concernant le Congo en nous interrogeant notamment sur les nouveaux types de villes que produit l'Afrique et sur les nouveaux types de citadins (Nicolaï H., 1994 b).

Un petit atlas fort utile (20 planches en format A4) donne des informations sur la localisation et la hiérarchie démographique et administrative des villes, l'évolution récente de leur population (les données de base sont le plus souvent de 1994 mais les commentaires, y compris pour les autres planches, fournissent quelques données plus récentes), leur structure par rang, la relation avec la densité rurale environnante, leur structure démographique comparée, leur niveau d'équipements, leur consommation en eau et électricité, la part du secteur secondaire et celle du secteur tertiaire, leur place dans les régions écologiques et dans le système des voies de communication, leur dépendance alimentaire, leurs infrastructures de santé et de scolarisation, l'origine de leur population. On y trouvera aussi des schémas de l'organisation urbaine de Kinshasa, Lubumbashi, Mbuji Mayi et Kisangani. Le commentaire de chacune des vingt planches est suivi de recommandations sur les interventions éventuelles à envisager (Wolff E. et al., 2001).

Depuis 1960, la ségrégation coloniale qui existait dans les villes congolaises s'est transformée en ségrégation sociale. Dans les années 80, il y a eu tendance au développement spontané d'un habitat mixte où pauvres et riches vivent côte à côte, par suite de l'envahissement par les premiers des espaces verts des quartiers riches. La gestion foncière est devenue «spontanée». On estime qu'un quart seulement des parcelles foncières a fait l'objet d'un enregistrement réglementaire (Lelo Nzuzi, 1992).

Un document à verser au dossier des ségrégations ethniques des groupes minoritaires. Les Tetela, à Kananga, se regroupent dans quelques quartiers, utilisent le même pôle commercial péricentral et se distinguent par la pratique de la riziculture (Kabamba K. et Lukenga C.E., 2002).

\section{L'APPROVISIONNEMENT VIVRIER}

Sur le thème de l'approvisionnement vivrier, qui avait été développé assez fortement avant 1990, de nouvelles publications prolongent généralement des études antérieures comme par exemple la grande enquête sur la commercialisation des produits vivriers à Kinshasa dirigée par Eric Tollens dont il a été largement rendu compte dans notre chronique précédente (Nicolaï H., 1993) et qui avait été menée de mars 1987 à juin 1990. Elle est synthétisée dans un gros livre qui analyse la structure et la performance du système de commercialisation ainsi que les comportements des acteurs, les conditions physiques et institutionnelles, la production agricole dans le bassin d'approvisionnement (Bas-Congo, Bandundu et autres régions du Congo), les revenus paysans, la collecte et le transport, la fluctuation saisonnière des prix, la distribution à Kinshasa et la consommation. On y trouve une estimation de la performance de la chaîne de commercialisation. Les auteurs croient qu'une amélioration de celle-ci aurait des effets positifs sur la production et la consommation et font quelques recommandations en ce sens (Goossens F., Minten B., Tollens E., 1994). Mais E. Tollens s'interroge sur la possibilité pour ce système d'entraîner un véritable développement économique, notamment dans le cas des commerçants «par colis», c'est-à-dire qui louent une place sur un camion pour eux-mêmes et la marchandise qu'ils sont allés acheter dans la région rurale. Le système est peu efficace à cause de la durée des transports, des faibles quantités négociées par commerçant, des mauvaises conditions de 
conservation des produits et il évoluerait difficilement vers une économie formelle (Tollens E., 1994). Une étude prolonge cette enquête en insistant sur le marché du manioc et analyse le comportement des acteurs de ce marché (Goossens F., 1996). Voir aussi, pour la même époque, un article dans une revue française (Rapoport H., 1993).

A Kinshasa, quelques années plus tard, la situation n'a guère évolué. La sécurité alimentaire n'est assurée que grâce au recours à des activités d'agriculture et d'élevage urbains ou périurbains: élevage de poules pondeuses, maraîchage, transformation et commercialisation du manioc. Cela apparaît nettement dans les budgets de consommation des ménages à partir de relevés faits dans trois quartiers pauvres (Mukadi Kankonde et Tollens E., 2001). Cependant malgré les crises politiques, on ne peut pas dire que la situation se soit vraiment dégradée. En fait le problème d'approvisionnement en tant que tel a fait place à un problème de pauvreté. L'alimentation absorbe 39\% du revenu familial dans les communes les plus pauvres. II y a eu crise du manioc. De nouveaux produits de culture et de consommation se sont développés comme le niébé. L'approvisionnement par bateau depuis la province de l'Equateur et la Province Orientale a pratiquement cessé. Par contre, il a augmenté fortement à partir du Bandundu par suite de la dégradation du réseau routier (Tollens E., 2004).

Une étude plus modeste décrit les modes d'approvisionnement en produits vivriers de Kisangani. Les commerçants citadins prennent le camion pour aller se ravitailler dans les marchés ruraux le long des routes vers l'intérieur jusqu'à une centaine de kilomètres. Mais on utilise de plus en plus le vélo en raison de la diminution du nombre de camions et de camionnettes, conséquence de la crise et aussi de la paupérisation de nombreux consommateurs. En outre, en raison de l'état des routes, beaucoup de villageois apportent leurs produits en pirogues sur la Tshopo, la Lindi et le Congo. Des marchés sont apparus ainsi dans l'agglomération sur les berges de ces cours d'eau. Quelques- uns sont de véritables villages flottants (Baya Ki Malanda, 1999).

Des articles étudient l'approvisionnement en produits spécifiques. Ainsi, au début des années 1990, la viande consommée à Kinshasa venait pour $35 \%$ du NordKivu, pour $25 \%$ du Bandundu, pour 17\% du Bas-Congo et pour $15 \%$ de l'Ituri. On notera que du Bandundu, une partie de la production arrive à Kinshasa par «baleinières» artisanales (grosses pirogues de $5 \mathrm{t}$, avec deux moteurs hors-bord, qui peuvent charger une cinquantaine de bovins de 300 kg). De 1978 à 1990, il y a eu accroissement sensible de la production ( $3 \%$ par an) grâce à de grands projets soutenus par des organismes internationaux (FAO, PNUB, BIRD) ainsi que par le Canada et la France. La bourgeoisie urbaine (grands commerçants, hauts et moyens fonctionnaires, hommes politiques) a investi dans les grands élevages, bon placement en raison de l'instabilité monétaire. Mais, notamment par suite des coûts élevés du transport, la viande reste trop chère pour la grande masse de la population. La consommation individuelle a baissé. Les viandes importées, de second choix mais de moindre prix, font une forte concurrence à la viande indigène. La concurrence la plus forte vient du poisson congelé importé (le mpiodi c'est-à-dire du chinchard, poisson ressemblant à du maquereau mais de plus faible qualité) dont le prix est beaucoup plus bas (Nzeza K.Z.K. J.-P., 1999).

Le commerce des huiles végétales sur les marchés de Lubumbashi est un bon exemple des circuits commerciaux des produits vivriers. L'huile de palme arrive, par la voie ferrée, du Bandundu, du Kasai, du Maniema et du nord du Katanga, l'huile de maïs est importée de Zambie par la route. Pléthore d'intermédiaires. Le bidon d'huile n'est accessible qu'aux ménages aisés. Les autres achètent des bouteilles d'un demi-litre. Aussi les prix sur les marchés des quartiers pauvres, où la vente se fait au microdétail, sont-ils aussi élevés (sinon plus élevés) que sur les marchés des quartiers aisés (Assani A. et al., 1993).

Les vendeurs en dehors des marchés. De 
nombreux petits vendeurs attirés par les marchés s'installent tout autour. Ces vendeurs à la sauvette récupèrent parfois les déchets des marchés. Leurs produits, qui sont donc généralement de mauvaise qualité, posent un problème sanitaire (Kayembe C.P.M., Kakese K.B., Solotshi M., 1993).

La production vivrière de la ville. Une part non négligeable des aliments consommés dans la ville provient de la ville elle-même. Les Kinois par exemple ont généralement plusieurs arbres fruitiers dans leur parcelle (2, 5 en moyenne à Limete). Dix-neuf espèces ont été répertoriées à Limete, soit environ 47000 pieds. Les six espèces principales sont le manguier, l'avocatier, le palmier à huile, le papayer, le safoutier et une espèce non ligneuse, le bananier (que les auteurs classent parmi les arbres!). Elles produisent un peu plus de 4000 tonnes de fruits par an. II semblerait que la part relative de ces espèces varie au sein des différents quartiers de l'agglomération. On pourrait certainement accroître la production (Makumbelo E. et al., 2005 et 2002)

Une autre part de l'alimentation de base est produite par les citadins eux-mêmes, dans les espaces périurbains. Un groupe de maraîchères de Kinshasa, à majorité manianga (originaires du territoire de Luozi, dans le Bas-Congo; elles ont pris le relais, après 1954, des femmes Lari, groupe très proche mais du CongoBrazzaville) a été étudié dans une thèse de doctorat, Elles ont leurs potagers dans la commune de Ndjili (44 jardins) et dans la vallée de la Funa (60 jardins) en 1989 et 1990. De petits bâtiments en briques sont occupés par des gardiens de nuit. Certaines maraîchères ont une activité spécifique de pépiniéristes. Ces femmes assurent elles-mêmes la commercialisation de leurs produits (les hommes ne sauraient pas fixer les prix!) mais en associant cependant les hommes à la gestion de leurs revenus (pour ne pas nuire à l'image de leurs maris!). Elles veillent à assurer la transmission de leur propriété à leurs enfants et à en écarter la famille de leurs maris (en veillant cependant à se protéger des actes de sorcellerie que celle-ci pourrait commettre à leur égard). Ces femmes maraîchères sont donc des «acteurs économiques» importants, qui gèrent de façon autonome leurs entreprises, préservent bénéfices et capital des convoitises masculines et participent ainsi à un développement endogène, c'est-à-dire s'appuyant sur les ressources internes d'un groupe. Mais il n'est pas certain, cependant qu'elles changent en profondeur l'ordre établi par les hommes (Mianda D.M.G., 1996). Signalons en passant que la même auteure discute ce dernier problème dans une revue canadienne et ne croit pas à une réelle émancipation de la femme sous le régime mobutiste (Mianda D.M.G., 1995).

Soixante-cinq pour cent des légumes mis en vente et consommés dans l'agglomération de Kinshasa sont produits ainsi dans la ville même et dans sa périphérie immédiate. La pauvreté des sols essentiellement sableux exigerait l'utilisation d'engrais que les producteurs sont incapables d'acheter. II faudrait développer la production de compost utilisant les déchets domestiques, les déjections d'élevages de porcs et de poulets mais aussi les sous-produits d'industries urbaines (brasseries, usines de traitement du café), les gadoues, etc. (Boeckx P. et al., 2006).

Voici une étude préliminaire sur l'approvisionnement vivrier de Kikwit. On remarquera qu'une part importante arrive aujourd'hui à la ville par la rivière (Mpuru M.B., 1993).

A Kananga, des points de vente périphériques, qui sont soit des marchés hebdomadaires, soit des points de vente flottants installés à des carrefours ou à des ponts, sont des lieux où des consommateurs urbains ou des vendeurs des marchés urbains viennent rencontrer des producteurs-vendeurs de l'arrière-pays immédiat, c'est-à-dire d'un espace rural situé entre 15 et $30 \mathrm{~km}$ du centre-ville. Une enquête a été menée dans sept d'entre eux notamment pour délimiter l'aire de résidence de leur clientèle (Kabamba Kabata et Nyoka Mupangila, 1998).

Dans les villes petites et moyennes, la quasi-totalité des ménages utilisent des combustibles végétaux. A Kananga, on a évalué en 1998 à 595 tonnes la quantité 
totale de charbon de bois entrant chaque mois dans la ville. La plus grande part arrive par la route de Mbuji Mayi dont la moitié par vélo. Dans la forêt de Muanzangoma, au nord-est de la ville, la production de charbon de bois est l'activité principale de la population. La zone d'approvisionnement ne s'étend pas audelà d'une trentaine de kilomètres. $28 \%$ des ménages utilisent directement le bois qu'ils vont récolter eux-mêmes car ils n'ont pas les moyens d'acheter du charbon de bois sur les marchés. L'électricité n'est utilisée que dans une toute petite partie de la ville (dans la commune de Kananga même) (Bushabu M.-M., Mabira M. et Kapend M., 2002) Dans le cadre des relations ville-campagne, un article analyse les flux de véhicules motorisés entre Kananga et son arrière-pays immédiat (Ntumba K., 1999).

\section{L'EFFERVESCENCE DE LA VIE RELI- GIEUSE}

C'est dans les villes surtout que se manifeste ce que I'historien Ndaywel considère comme l'amorce d'une «troisième évangélisation» du Congo. Le groupe de prière (fraternité de prière) est devenu un lieu de mobilisation par excellence des citadins et apparaît comme une recette de survie. Le religieux envahit la ville et ses manifestations. La chanson religieuse est en passe de concurrencer la chanson de cabaret. Les espaces de prière ont fait leur apparition dans les communautés congolaises en Europe. Ndaywel parle même d'un climat de "surchristianisation» caractérisant «une volonté unanime d'assainissement moral de l'ensemble de la vie nationale» (Ndaywel I., 1993).

Cette «surchristianisation» se manifeste dans le paysage urbain de Kinshasa par la multiplication des lieux de culte, un au moins pour dix parcelles d'habitation (Kakese C., 2000). Armée de Victoire, Armée de l'Eternel, Restauration, Rachetés de Jésus-Christ, Arche de Noé, Manne cachée, Eglise de la Nouvelle-Jérusalem, etc., tous ces groupes sont fidèles à un type pentecôtiste issu du monde outreatlantique, surtout des Etats-Unis où la réussite matérielle apparaît comme une manifestation de l'amour de Dieu. Le Pentecôtisme a ainsi promu l'individualisme, donc une attitude envers le travail et l'argent, en opposition avec l'identité ethnique antérieure. Un phénomène inquiétant: l'attribution de beaucoup d'enfants des rues à la catégorie des enfants-sorciers, rangés dans le monde des démons (Elongo Likulunga V., 2002). Matangila rappelle qu'une enquête récente a dénombré plus de 3000 groupes de prières ou d'Eglises de réveil dans l'agglomération de Kinshasa dont 391 étaient régulièrement enregistrés par la Ligue des Eglises et Ministères de Réveil au Congo. Leur discours répond à un certain nombre d'enjeux: le salut de l'âme, la santé du corps, la protection contre les mauvais esprits, le bien-être matériel et social des conducteurs du groupe et de leurs adeptes et assume des fonctions messianiques, prophétiques et thérapeutiques (Matangila A., 2006).

L'anthropologue René Devisch, qui suit depuis des années des groupes de population yaka qui vivent à Kinshasa, a créé une expression originale pour décrire les effets de la crise, après les épisodes de pillages de septembre 1991 et janvier 1993. "Misère et incohérence de la vie, écrit-il, brisent les modèles postcoloniaux de progrès et les miroirs d'identification». Comme les citadins continuent à porter au fond d'eux-mêmes la loi parentale et ancestrale établie au village, au moins en tant que principes de perception et d'appréciation, ils cherchent refuge dans une solidarité de voisinage (une «villagisation" de la ville) et dans les églises de guérison, avec aussi un repli sur l'espace autour des mères (marché, maison, champs) (Devisch R., 1994 et 1995).

L'implantation des églises de guérison charismatiques et prophétiques dans toutes les couches de la société comble ainsi d'une certaine manière le vide éthique et législatif creusé par la désertion des tribunaux et la dissolution du parti-Etat. R.Devisch en a étudié la filiation à partir des enseignements des missionnaires protestants en pays kongo, qui accordaient une grande importance au Saint-Esprit. L'image du Saint-Esprit a été rapprochée de celle de l'esprit ancestral. 
Les églises de guérison ou de l'Esprit Saint sont des communautés charismatiques réunies autour d'un homme ou d'une femme considéré comme leur prophète ou leur chef spirituel. Elles ont repris les traditions des églises kimbanguistes et protestantes en ce qui concerne la Bible, les chants et les manuels de prières. Plutôt que de viser à transmettre l'Evangile, les prophètes ont pour tâche d'aider et de guérir les gens. L'union au Saint-Esprit donne lieu à des liturgies qui durent de cinq à six heures, avec parfois des épisodes à la limite de la violence. Ces églises auraient ainsi indirectement contribué au soulèvement populaire de décembre 1990 et de septembre 1991 parce qu'il s'agissait d'une réaction aux idéaux et aux modes de vie postcoloniaux et capitalistes, axés sur une consommation ostentatoire. Mais en promettant un nouvel ordre du monde et en donnant aux gens, par leur encadrement, la conscience d'avoir une certaine prise sur la vie publique, au moins dans leur voisinage, elles auraient peut-être désamorcé les risques d'explosions catastrophiques comme en ont connues le Rwanda, l'Angola et le Soudan (Devisch R., 1996). Les femmes y jouent un grand rôle (Devisch R.,1999)

Les nouvelles Eglises interviennent dans les soins de santé. A côté des guérisseurs initiés ou improvisés pratiquant des formes variées de soins, à côte de services médicaux modernes, Pentecôtistes et églises de guérison indépendantes pratiquent le traitement charismatique des maladies par la foi (Van Wolputte S., Devisch R., Le Roy J. et Lapika D., 2002). L'annonce de l'apocalypse. L'idée que la vie quotidienne actuelle, avec ses problèmes et ses malheurs, annonce la fin des temps, est présente dans une partie importante de la population de Kinshasa. Celle-ci y voit la mort d'une société dominée par le Mal (De Boeck F., 2001d). Cependant tout ce qui concerne la mort échappe au contrôle des Eglises de réveil. Les funérailles s'accompagnent souvent de manifestations de contestation politique, dans les chants notamment, surtout de la part des jeunes. Les «Ecuries» de jeunes jouent un rôle impor- tant dans leur organisation. Les funérailles ne sont plus l'affaire unique des vieux comme jadis mais les jeunes y prennent une part plus grande. La mort est devenue le moteur de toute une économie informelle nouvelle (De Boeck F., 2006).

Le rapport à la mort se présente-t-il de façon particulière dans les villes? II est certain déjà que, dans le Congo actuel, la relation à la mort telle qu'elle s'exprime par exemple dans les rites funéraires n'est plus la même que dans la société traditionnelle. La crise économique et politique, en aggravant la situation des gens, ajoute ses propres perturbations. Outre une communication (T.K. Biaya) portant sur la façon dont les artistes, peintres et chanteurs, représentent le thème de la mort, on trouvera, dans un livre dirigé par Grootaers J.-L. (1998), une étude, par ce dernier, des enterrements et des cimetières à Kinshasa à partir des articles de la presse locale. Le prix exorbitant d'un enterrement empêche d'inhumer dignement ses morts. La désaffectation de fait de certains cimetières transformés en potagers urbains ou en lotissements pose aussi des problèmes. Une communication décrit les rites funéraires classiques et les rites des jeunes (par G.M. Kamandji). De ces deux communications, on retiendra notamment que la difficulté à accomplir les rites traditionnels engendre la crainte d'une zombification des morts qui viendraient dès lors hanter les vivants. On trouvera aussi des exemples assez hallucinants sur les pratiques de séquestration des nouveauxnés et des cadavres par les institutions hospitalières, tant publiques que privées, pour essayer de recouvrer les honoraires et frais non payés. Le ministre de la santé a dû les interdire par une circulaire officielle. On lira aussi la tendance des jeunes, lorsqu'un des leurs meurt (par exemple du sida), à poursuivre, souvent dans la famille du mort, celui ou celle qui serait le sorcier responsable du décès. Ils n'hésitent pas non plus à racketter les passants et les automobilistes pour se procurer l'argent nécessaire aux funérailles. Dans un important chapitre, De Boeck F. (1998), à côté de réflexions théo- 
riques sur ce sujet, fournit nombre de faits éclairants. L'épidémie de la fièvre Ebola, qui a fait plus de 200 victimes en 1995 à Kikwit, illustre bien le désarroi des personnes concernées. D'abord dans les explications qui en font le produit d'une sorcellerie moderne: vengeance d'un missionnaire américain chassé parce qu'il se transformait la nuit en hippopotame dans la rivière Kwilu pour effrayer la population ou bien vengeance de celui qui était chef du village de Kipuka (un des sites de l'actuel Kikwit) à l'arrivée des colonisateurs et qui avait résisté à leur intrusion, vengeance contre ceux qui auraient prélevé de la terre sur sa tombe. Ensuite sur le sort de ces corps emballés dans du plastique et inhumés, dans des fosses anonymes par des gens euxmêmes habillés de combinaisons étanches. Une intéressante description aussi de ces sortes de revenants que sont certains Bana Lunda, jeunes gens partis chercher du diamant en Angola et rentrés au pays avec des marques sur le corps: marques de coups de fouet ou de blessures par balles ou éclats d'obus, membres amputés par l'explosion d'une mine, oreilles coupées par les combattants de l'UNITA ou les soldats du MPLA. Sur cette question, voir aussi De Boeck F., 1999.

\section{LES ÉTUDES SUR LES GRANDES AGGLOMÉRATIONS}

La plupart des exemples de problèmes urbains, qui ont été cités dans les paragraphes précédents, ont été pris dans les grandes agglomérations et plus précisément à Kinshasa et à Lubumbashi. Nous allons examiner maintenant les études qui concernent plus précisément des aspects spécifiques de ces agglomérations.

\section{Kinshasa}

- L'expansion de l'agglomération et les données socio-démographiques

A l'occasion du quatre-vingtième anniversaire de son accession au rang de cheflieu du Congo, voici un album de photo- graphies illustrant l'histoire de la ville (Eale B. J.-Cl., 2004)

L'utilisation d'images satellitaires SPOT de 1995 a permis de délimiter l'extension spatiale de l'agglomération de Kinshasa et de calculer la densité de population par rapport à la surface bâtie. La comparaison avec la situation de 1969 montre que l'extension spatiale s'est accompagnée aussi d'une densification de l'espace bâti qui a absorbé près des deux tiers de l'accroissement de la population (Delbart V. et Wolff E., 2002).

En 1995, malgré une tendance à la baisse de la fécondité, la taille du ménage est plus grande à Kinshasa que dans le milieu rural $(7,7$ membres contre une moyenne nationale de 5,4). Elle est en augmentation continue depuis 1974 marquant ainsi une évolution vers un ménagerefuge, conséquence de la crise de l'emploi et de celle du logement. II y a cohabitation des enfants mariés avec leurs parents. Le vieillissement de la structure de la population et le retard de l'âge au mariage annoncent une transition vers une plus faible fécondité. Les personnes de moins de 15 ans qui faisaient $50,3 \%$ de la population totale en 1974 ne font plus que $42,4 \%$ en 1995. La part des adultes est passée de 48,5\% en 1974 à $56,1 \%$ en 1995 (Ngondo a Pitshandenge, 1996).

Les enquêtes que L. de Saint Moulin organise sans relâche avec ses étudiants depuis de nombreuses années, dans différentes communes de Kinshasa, sont une mine d'informations originales dans des domaines variés. Nous en citons quelques-unes ici. Elles ont été publiées presque chaque année dans la revue Congo-Afrique, à laquelle nous renvoyons le lecteur. Le compte rendu de l'enquête fournit le plus souvent les données sociodémographiques des quartiers étudiés. Comme certains de ceux-ci ont été revisités à plusieurs années d'intervalle, on peut suivre ainsi l'évolution de ces données. Si les thèmes étudiés peuvent parfois paraître éloignés des préoccupations des géographes, les réponses aux questions posées sont souvent très instructives et révèlent des faits ou des facteurs dont on ne soupçonnait pas toujours l'importance. Voici comme exemple d'une 
enquête qui fournit indirectement des indications sur les changements socioéconomiques survenus dans la population en trois décennies, celle sur la perception de la justice (54\% des Kinois estiment que c'est dans l'exercice du pouvoir par les gouvernants qu'il y a le plus d'injustice). L'étude a porté en effet sur un échantillon construit de la même façon que celui qui avait été utilisé pour l'enquête menée par le même auteur en 1967: 1175 personnes réparties dans les différents quartiers de l'agglomération, sauf Nsele et Maluku. Les célibataires, par exemple, qui représentaient $25,6 \%$ des plus de 17 ans en 1967 sont passés à un peu plus de $50 \%$ en 1996. L'allongement des études et l'augmentation du coût de la mise en ménage ont retardé l'âge du mariage. Les niveaux d'études ont fortement augmenté: $8 \%$ d'hommes et $3 \%$ de femmes ayant fait des études supérieures en $1967,38,2 \%$ et $20,8 \%$ en 1996 . Le pourcentage des sans emploi parmi les hommes n'a pas sensiblement varié (un peu plus de $13 \%$ ). Par contre, il s'est fortement réduit chez les femmes $(29,9 \%$ en 1996 contre $92,4 \%$ en 1967). La part des catholiques et des protestants (et même des kimbanguistes) s'est réduite, respectivement de 6,4 , de 3,6 et de $4,5 \%$, au bénéfice des membres de multiples sectes chrétiennes $(+12,1 \%)$ et d'autres religions comme l'islam (de Saint Moulin L., 1996a). Une enquête portant sur différentes perceptions (utilité de l'instruction, causes de la délinquance, application de la justice, etc.) fournit des renseignements socio-démographiques de même type pour le quartier de Kindele, qui s'est développé le long de la route de l'Université à Kimwenza (de Saint Moulin L., 1996 b). II en est de même pour une enquête ultérieure menée dans la commune de Ngaba sur la perception de la mondialisation. Dans ce quartier modeste, $25 \%$ des hommes et $10 \%$ des femmes ont un diplôme d'études supérieures. Remarquons que l'idée d'une participation à la mondialisation comme voie obligée du développement y a été approuvée par $42,7 \%$ des enquêtés (il n'y a eu que $29,8 \%$ d'avis défavorables; $17,7 \%$ y ont vu aussi un moyen de dépasser les problèmes eth- niques). 60,1\% sont d'avis cependant que les étrangers exploitent le pays ou empêchent son vrai développement (de Saint Moulin L., 1998). Dans une autre enquête de ce type, en 1999, portant cette fois sur la perception des identités et des responsabilités sociales, dans un quartier voisin lui aussi de l'université, on trouvera d'intéressantes réponses (mais pas toujours inattendues) à des questions comme «où est-il préférable de trouver du travail?» chez le Blanc: 31,4\%, dans les services publics: $34,3 \%$, comme indépendant dans les activités informelles: 30,3\%, chez quelqu'un de son ethnie: $5,6 \%$ ou bien «pourquoi les jeunes s'enrôlent-ils dans l'armée?» pour avoir un emploi: 43,6 \% (de Saint Moulin L. et Kabongo Mpunga, 2000).

- Les activités commerciales et les marchés

Dans le cadre de ce thème de la géographie urbaine africaine, qui a été souvent privilégié, Kanene analyse ce qu'elle appelle le commerce «sédentaire» représenté surtout dans le centre-ville où elle distingue 1) le commerce européen, 2) le commerce libano-pakistanais, qui a supplanté, après 1960, le commerce des Grecs et des Portugais, 3) le commerce zaïrois qui fait seulement $11 \%$ des commerçants du centre-ville. De nombreux commerçants-transporteurs assurent le ravitaillement de la ville en produits alimentaires régionaux. Des femmes commerçantes internationales contrôlent le commerce des wax, des chaussures, des bijoux et de l'électro-ménager. L'article étudie aussi la genèse, la localisation et la structure des marchés (585 au total) et les relations entre les marchés et les commerçants "sédentaires» (Kanene Mpali Sitela, 1992). Ndjili la première cité satellite de Kinshasa, planifiée à la fin de la période coloniale sur la route du nouvel aéroport, s'est fortement agrandie par la suite en englobant et transformant les anciens villages de Kibanseke et de Macina pour atteindre une population estimée à 900000 habitants en 1990. Elle compte de nombreuses exploitations maraîchères dans la vallée de la Ndjili 
(voir plus haut le livre de Mianda G.). Ndjili fonctionne aujourd'hui comme le noyau central de la partie orientale de l'agglomération de Kinshasa. On y trouve deux marchés de gros pour les produits agricoles arrivant des régions rurales, essentiellement du Bandundu (Makumaya Nsamba Kiambamba, 1992).

- La pauvreté urbaine et les stratégies de survie

Un thème particulièrement développé dans les études urbaines est celui de la pauvreté. II est abordé dans des enquêtes menées par des organismes très divers. Une d'entre elles, exécutée en 1997, avec l'appui du PNUD et de I'UNICEF, a porté sur un échantillon de 1970 ménages (environ 16000 personnes). La taille moyenne du ménage est de 8,1 personnes (7,7 dans l'étude de Ngondo Pitshandenge citée plus haut), avec un logement de 2,5 chambres. $21 \%$ des chefs de ménage sont des femmes, 11 \% sont au chômage, 43 \% vivent du commerce informel, qui fournit un revenu moyen mensuel de 102 US \$. Parmi ceux qui ont un revenu, seuls $10 \%$ le considèrent comme suffisant. $80 \%$ des ménages sont raccordés à l'eau potable, 60 \% à l'électricité, $21,6 \%$ seulement ont accès aux soins de santé. Contrairement à ce qui apparaît dans une l'étude de Cordaid commentée plus loin, $72,1 \%$ des ménages se considèrent effectivement comme pauvres. Cette perception est très proche des données statistiques. En effet si l'on prend, comme seuil de pauvreté, une dépense journalière individuelle de $1 \$$, $78,5 \%$ des ménages sont pauvres (Mususa Ulimwengu J., 1998).

Voici une autre évaluation de la pauvreté faite en 1994 dans 4 des 51 divisions de la zone de Matete. Composition moyenne des ménages, 7,7 personnes (avec un maximum de 18 et une valeur modale de 15). Pour la consommation de calories, $80,6 \%$ de la population est en dessous de la limite de 2960 calories, 42,1\% en dessous de la norme de 2200. Si l'on considère le volume des dépenses, $61,3 \%$ de la population peuvent être considérés comme pauvres. 93,5\% des ménages consacrent plus de $50 \%$ de leurs dépenses à l'alimentation, ce qui est un autre critère de pauvreté (D'Zouza S., Makaya Mboko Mbenza et Kalendi Muhindo, 1995)

Un autre livre sur la pauvreté à Kinshasa a été réalisé par des chercheurs africains, avec le soutien d'une ONG (Cordaid), en grande partie néerlandaise, à partir des éléments d'une enquête menée dans les communes de Lingwala et de Ngaliema en avril 2003. L'étude analyse les caractéristiques socio-démographiques des ménages enquêtés, le cadre et la qualité de vie (nombre de chambres à coucher, nombre de personnes par chambre, statut d'occupation - l'échantillon comporte $49 \%$ de propriétaires -, raccordement à l'eau et à l'électricité, présence de latrines, évacuation des déchets), la vie quotidienne des ménages, l'accès à l'éducation et aux soins de santé, la fréquence et le volume des conflits (très faibles). Elle s'interroge sur la perception de la pauvreté par les habitants et les stratégies mises en oeuvre pour en sortir. En fait on ne se considère pas comme pauvre mais comme momentanément «en manque». Les habitants de Ngaliema apparaissent plus pauvres que ceux de Lingwala. L'étude s'interroge aussi sur le rôle que I'ONG qui a organisé l'enquête pourrait jouer pour relever le niveau de vie. On remarquera que peu de personnes enquêtées connaissent son action (Lelo Nzuzi F. et Tshimanga Mbuyi Cl., 2004).

Un autre livre sur la pauvreté, publié lui aussi en 2004, exprime un point de vue plutôt économique. II est le résultat d'une Table ronde organisée par l'Observatoire d'Economie et de Développement humain, unité de coopération scientifique réunissant la Faculté d'Economie et Développement des Facultés Catholiques de Kinshasa (FCK) et les Facultés Universitaires Saint Ignace d'Anvers (UFSIA) (Vivre et survivre à Kinshasa, 2004). II s'agissait de faire le point au sortir de cinq années de guerre succédant aux pillages de 1991 et 1993. La plupart des études ont concerné la commune de Kisenso, certaines en vue d'accompagner le fonctionnement d'un projet de 
coopération, le Fonds Social Urbain. Parmi les nombreux thèmes traités, citons une analyse multidimensionnelle de la pauvreté dans les ménages de Kisenzo, par Cl. Tshimanga (pp. 181-180) et une étude de l'évolution de la pauvreté monétaire de 1997 à 2002, par T. de Herdt, dans laquelle l'auteur cherche à construire un indicateur de la pauvreté monétaire basé sur des données d'enquêtes budgétaires. La reprise de l'exode vers la ville, à cause de la guerre, a exercé une forte pression sur le marché immobilier. De moins en moins de personnes sont propriétaires de leur logement. On renonce de plus en plus à utiliser l'électricité pour la cuisine, ce qui a entraîné par ricochet, une augmentation du prix du bois. On notera aussi une étude de la situation socio-économique des «navetteurs» de Kisenso, avec une foule de tableaux statistiques dont certains sont pleins d'intérêt mais d'autres parfois apparaissent peu utiles (par Lelo Nzuzi F., Dickens E., Loka Kongo et Mvubu Ntoto). Une communication fait le bilan de l'impact du Fonds Social Urbain sur la qualité de vie. Des améliorations sont incontestables. Le FSU a soutenu 44 «Initiatives Locales de Développement» dans le secteur du drainage et des voiries, la lutte anti-érosive, la réhabilitation d'écoles, l'appui à des activités génératrices de revenus (vendeuses de pain, couturières, maraîchage, élevage de volaille, de porcs, etc.), l'aménagement de points d'eau, de latrines, les programmes de formation pour 130 ONG. Mais que deviendra tout cela quand le projet se terminera (sa durée prévue était de quatre ans)?(Kamavu Dungo). Notons aussi une mise au point sur les petites et moyennes entreprises de production dans la commune de Kalamu (Ali Emedi A). Certaines communications étendent leurs préoccupations à l'ensemble du Congo, comme celle sur la «pauvreté féminine et sécurité alimentaires des ménages en RDC» (Bambila Mulumba $\mathrm{H}$.). Une autre traite le cas de l'accessibilité aux soins de santé dans la commune de Ngaba (Kebela Kebela P.). D'autres envisagent des stratégies de survie spécifiques.

On trouvera une étude de la structure et des déterminants du budget de consommation des ménages dans les quartiers pauvres de Kisenso, Kindele et Makala dans l'ouvrage de Kankonde Mukadi et Tollens E., cité plus haut (N'toto M'vubu, 2001).

Voici une description pittoresque et le vocabulaire des petits métiers de l'informel: mamans bipupula (qui grattent le sol des marchés), bana cirage (cireurs de chaussure), kadhafi (vendeurs d'essence au détail), bana cabria (rôtisseurs publics), ba cambistes (changeurs de monnaie), bana-Koweit (cambrioleurs en liaison avec les receleurs du marché), bana mayi (vendeurs d'eau ambulants), dactylographes (écrivains publics), etc. (Lukeso P., 2005).

Dans l'ouvrage Vivre et survivre à Kinshasa, une communication analyse tout particulièrement, dans le secteur du petit commerce, le fonctionnement des liglabos, c'est-à-dire des échoppes mobiles, sans local fixe, les stratégies développées par les boutiquiers, y compris les petites fraudes sur les poids et mesures et les procédés à la limite de la magie pour attirer la clientèle. II semble que l'auteur ne mette pas vraiment en doute l'efficacité de ces derniers. II conclut d'ailleurs qu' «il y a un «mystère» dans cette façon congolaise de survivre, on dirait qu'il y a une puissante main invisible qui soutient la traversée tumultueuse de la pirogue congolaise devant les tempêtes dévastatrices dans cet océan de la misère vers des horizons inconnus». Ce n'est pas la première fois que nous rencontrons dans des ouvrages scientifiques, ou que nous avons entendu dans des réunions scientifiques, y compris des soutenances de thèse, des réflexions surprenantes de ce type (l'auteur n'hésite pas à la souligner dans le texte) sur la prétendue puissance d'une magie africaine qui expliquerait ce que le chercheur ne peut expliquer (Ngunu Kasai D.P., 2004).

Pourrait-on vivre uniquement des ressources d'une activité agricole dans la ville? L'analyse comptable de plus de 300 exploitations de divers quartiers de Kinshasa (120 exploitations maraîchères produisant de l'amarante, 40 exploitations 
de tilapia, 60 exploitations porcines, 120 exploitations de poules pondeuses, 3 exploitations de champignons), arrive à la conclusion qu'aucun exploitant n'arrive à rentabiliser son exploitation (la vente de la production ne couvrant pas les frais fixes) parce que celle-ci est trop petite, parce que le niveau de formation de l'exploitant est insuffisant et qu'il manque de crédit. Mais peut-on croire qu'il s'agisse vraiment d'un simple passe-temps? De tels résultats ne dépendent-ils pas aussi du système de comptabilité qui est utilisé? On notera, fait particulièrement intéressant, que de plus en plus d'hommes manifestent de l'intérêt pour le maraîchage (ils sont $80 \%$ dans l'échantillon retenu), que beaucoup d'exploitants ont un niveau d'instruction élevé (30\% des éleveurs de poules pondeuses ont fait des études supérieures ou universitaires) (Kinkela Savy et Mpanzu Balomba, 2004).

Pour lutter contre la pauvreté, le microcrédit (épargne et crédit) est souvent préconisé. Un projet de ce type, soutenu par la faculté catholique de Kinshasa et la faculté catholique d'Anvers, est discuté dans un colloque (Erpicum R. et Luzolele, 1998)

Theodore Trefon a rassemblé une douzaine de communications de 18 auteurs, congolais pour la plupart, sur le thème des réponses populaires à la faillite de l'Etat. Y sont envisagés différents aspects de la vie quotidienne: les activités de débrouille, l'approvisionnement en eau et en produits alimentaires, la santé publique, le rôle des ONG, les transferts d'argent des immigrés en Europe, l'évolution de la composition du ménage en rapport avec le déclin économique, les enfants de la rue et le monde occulte, les phénomènes de violence dans la musique et la politique, etc. (Trefon T., 2004). Dans une autre publication, T. Trefon analyse les aspects de la pauvreté dans la ville. Malgré un certain ralentissement de la croissance démographique, l'espace urbain et périurbain continue à être phagocyté par les habitations, les cultures vivrières, la destruction des forêts pour le bois de chauffe. Les espaces intraurbains disponibles sont utilisés pour les potagers. La ville se «rurali- se». La hausse considérable des prix du charbon de bois a entraîné une réduction du nombre de repas chauds. La viande de gibier devient de plus en plus rare. La voie d'eau prend une part accrue dans l'approvisionnement de la ville. On assiste à une surexploitation de la mosaïque écologique (Trefon Th., 2000).

Pour survivre, l'habitant de Kinshasa combine ainsi des comportements traditionnels et des comportements qui expriment sa vision de la «modernité». II utilise de multiples réseaux de solidarité: commerciaux, communautaires, religieux, parentaux. Mais, face à ses problèmes et à ceux du pays, il ne semble pas parvenir à transformer ses discours et ses attentes en une mobilisation politique (Trefon Th., 2002).

Un phénomène largement lié à la pauvreté est la prostitution. On trouve, à Kinshasa, en 1994, cinq catégories de prostituées: 1) les prostituées d'hôtels, de maisons de passe, 2) les prostituées de la rue (les «londoniennes», surnommées ainsi en raison de leur tenue à l'occidentale), 3) les prostituées des bicoques, les Mingando, généralement plus vieilles, dans les quartiers populaires, 4) depuis une dizaine d'années, les phazeures, adolescent(e)s qui passent la nuit en dehors du toit familial, dans la rue, des hangars, etc., 5) les prostituées clandestines (et occasionnelles), femmes mariées, étudiantes, écolières, etc.. L'information sur le sida est très déficiente dans tous ces groupes où l'analphabétisme est très fréquent (Nzilambi Nzila $\mathrm{E}$. et al., 1996).

Un autre fait caractéristique de la ville congolaise actuelle - et de nombreuses autres villes africaines - est l'abondance des enfants de la rue que les guerres civiles et les effets du sida ont multipliés. On trouvera quatre communications sur les enfants de la rue et les jeunes filles de Kinshasa et un sur les jeunes de la rue de Kisangani dans un ouvrage collectif consacré à ce phénomène en Afrique Noire (Marguerat Y. et Poitou D., 1994; Muwalawala K., Mukenz-Dibayam Kabn, Mnongompasi a'Nkuba, Mpey-Nka Ngubusim). De nombreux termes s'appliquent à ces enfants: moineaux, vaga- 
bonds, shege, enfants abandonnés. On distingue généralement les «enfants de la rue» qui passent leur vie dans la rue et ne gardent pas leur contact avec leur famille et les «enfants dans la rue» qui rentrent chez eux le soir. Avec la situation de guerre, sont apparus aussi les "enfants séparés» et les "enfants associés aux forces et groupes armés». Kakudji expose les méthodes qui lui ont permis de nouer des contacts avec ces enfants et de reconstituer leurs itinéraires de vie, dans l'est du Congo où l'UNICEF a monté un programme de recherche sur leur réinsertion (Kakudji A., 2006). Une grande menace pèse sur eux, celle d'être accusés de sorcellerie (De Boeck F., 2000) et rangés dans le monde des démons (Elongo Likulunga V., 2002). Sur le rôle des enfants dans la société urbaine congolaise actuelle, dans et hors des familles, et la question des enfants-sorciers, voir aussi les articles de De Boeck F. (2005) et De Boeck F. et Honwana A. (2000) et sur les shege de Kinshasa, ceux de Biaya Tshikala K. (2000 et 2005). Le numéro 80 (décembre 2000) de Politique africaine est consacré à ce thème (Enfants, jeunes et politique) qui englobe aussi celui des enfants-soldats (Honwana A., 2000). Sur les enfants-sorciers, voir aussi Tonda J., 2008.

Des bandes de jeunes transforment la rue en scènes de violence la nuit. Ces bakumbusu (gorilles en lingala) se livrent à des combats inspirés des gorilles, inventés semble-t-il dans la dernière décennie du Congo colonial, combinant une lutte traditionnelle des Mongo avec des pratiques de combat asiatiques et orientales. Elles proposent un modèle de masculinité différent de celui du sapeur et du staffeur (Pype K., 2007).

\section{- La mobilité urbaine}

Dans l'énorme agglomération qu'est aujourd'hui Kinshasa, et dans les conditions de précarité de ses habitants, les problèmes des transports urbains apparaissent difficilement solubles. Non seulement il y a sous-équipement des quartiers récents mais il y a dégradation de la situation des quartiers anciens. On comp- te seulement un autobus pour 8000 habitants. Mwanza ma Mwanza a soutenu une thèse sur ce sujet. II en a tiré un livre qui en reprend quelques parties et qui fournit notamment une vue historique très complète de la question. Après un rappel des tendances lourdes de la croissance urbaine et des structures socio-économiques, il analyse les rapports, études et projets techniques successifs ainsi que les études académiques. II a mené une enquête auprès des transporteurs urbains et a analysé les déplacements quotidiens en insistant sur les déplacements des écoliers qui en représentent une part importante (55\% des déplacements journaliers). II s'interroge sur l'échec d'une tentative de monopole public et décrit les stratégies d'adaptation de la population à la crise (Mwanza ma Mwanza, 1997). Dans des articles antérieurs, il avait décrit l'atomisation de l'offre privée et la baisse de la capacité (1995) et montré qu'une décentralisation des équipements scolaires pourrait contribuer à réduire quelque peu les problèmes de mobilité pour les écoliers. II avait proposé qu'une cellule spéciale soit chargée d'établir et d'ajuster à intervalles réguliers un canevas opérationnel (1996). Dans un article plus récent, il revient sur la nécessité, dans la période de crise que traverse le pays, de minimiser les déplacements scolaires. L'analyse des facteurs de choix des établissements scolaires montre que ces choix sont surtout qualitatifs. II faudrait donc améliorer la qualité des écoles des quartiers périphériques ou des quartiers défavorisés et, de façon générale, améliorer l'offre en services socio-collectifs (Mwanza wa Mwanza, 2000)

- Les problèmes de gestion urbaine et d'environnement

La gestion de Kinshasa pose de multiples problèmes: problèmes de pentes, problèmes de spéculation foncière, soit de petite spéculation, une famille achetant plusieurs terrains, soit de spéculation capitaliste lorsque l'achat porte sur de grands espaces encore libres, problèmes de services urbains (malgré les efforts 
faits dans certains quartiers), de transport, d'enseignement, de services de santé, problèmes de moyens pour les collectivités locales qui ont à leur charge toute une série de services, avec la circonstance aggravante que des services restant à la charge de l'Etat ne sont pas rendus, problèmes d'environnement. Très curieusement, dans les quartiers dits d'habitat spontané, l'insécurité foncière n'est pas aussi grande qu'on pourrait le croire, d'abord parce que l'administration n'a pratiquement aucune capacité de modifier les situations acquises, ensuite parce que les acquisitions foncières se font dans des cadres semi-traditionnels qui leur assurent une certaine garantie (Kankonde Mbuyi, 1993).

Nous avons évoqué déjà les problèmes typiques de l'environnement tropical urbain, celui des ravines d'érosion, à propos de Kikwit et d'Uvira (voir le chapitre consacré à la géomorphologie). Un livre d'un professeur de l'Université de Kinshasa l'envisage avec l'ensemble des problèmes environnementaux de l'agglomération. II évoque d'abord la façon dont une partie de la population aborde encore ces problèmes en leur attribuant des causes surnaturelles sur lesquelles elle n'aurait donc pas de prise (érosion déclenchée par des sorciers, inondations provoquées par des monstres vivant dans les fleuves et les rivières, maladies considérées généralement comme n'ayant aucun lien avec l'insalubrité du milieu). II rappelle les effets sur la végétation environnante de l'accroissement d'une agglomération, qui compterait 8,2 millions d'habitants en 2007. A l'intérieur du périmètre urbain, les arbres qui ne portent pas de fruits utilisés par la population sont condamnés à fournir du bois de construction ou du bois de feu. Certes, après un certain temps, les quartiers nouveaux se couvrent d'arbres fruitiers plantés à l'intérieur des parcelles mais, en dehors de celles-ci, tous les arbres non fruitiers disparaissent rapidement. L'auteur a établi personnellement plusieurs rapports demandés par le Ministère des Travaux publics sur les ravines d'érosion. Les mesures prises par les services techniques avec le soutien d'organismes internationaux n'ont le plus souvent qu'un effet éphémère. La seule méthode efficace serait d'empêcher l'installation anarchique d'habitations sur les pentes dépassant une certaine valeur (4\%?). La ville basse souffre d'inondations pendant la saison des pluies (crues des rivières dont le lit a parfois été encombré par les alluvions apportées par les ravines d'érosion, remontée de la crue du fleuve dans les régions les plus basses, remontée de la nappe phréatique, mais aussi rupture d'un bassin d'orage mal entretenu, etc.). Ces inondations peuvent causer en outre de gros dégâts aux cultures rizicoles pratiquées dans certaines régions basses. Un autre problème est l'absence d'évacuation des ordures ménagères. Des décharges non contrôlées se développent autour de chaque marché. Les ordures sont déversées dans les collecteurs d'évacuation des eaux ou dans les ravines (pour les combler et dans le but de lutter ainsi contre l'érosion?). Seuls quelques quartiers bénéficient d'une collecte organisée privée ou publique. Quelques ONG en organisent dans des quartiers populaires. Certes des chiffonniers récupèrent une partie des déchets, surtout le papier, et des ordures sont utilisées comme compost par les maraîchers mais cela est insuffisant. II faudrait une éducation de la population. L'auteur compte beaucoup sur les jeunes pour amorcer une prise de conscience des problèmes environnementaux et faire disparaître le sentiment d'une ville-poubelle, conséquence d'une mauvaise gouvernance et surtout de la pauvreté. Le livre contient beaucoup de détails pittoresques (Lelo Nzuzi F., 2008).

\section{- La vie et la culture urbaines}

Pour l'étude de la ville vue par ses habitants, les publications de l'équipe de l'anthropologue Filip De Boeck sont une source indispensable. Dans l'une d'elles, des anthropologues et un architecte discutent avec des Kinois (un écrivain, un psychiatre, un spécialiste des droits de l'homme, etc.) de la spécificité de l'urbanité en Afrique centrale et dessinent la géographie d'un imaginaire dans lequel 
la ville est mise en relation avec le corps, la sexualité, l'argent, la mort et la folie (Deboeck F., Van Synghel K. et Lombune Kalimasi V., 2005). Un autre livre illustre les imaginaires sociaux et culturels des habitants de Kinshasa. II reprend une partie des très belles photographies d'une exposition réalisée par ses auteurs, commandée par la Communauté Flamande et qui avait obtenu le Lion d'Or à la Biennale d'Architecture de Venise (De Boeck F. et Plissart M.-F., 2005).

Si l'on veut sentir le pouls de l'agglomération kinoise, écouter les rumeurs et percevoir jusqu'aux plus humbles des problèmes de la vie quotidienne, on lira les recueils des chroniques publiées dans des périodiques de la capitale et quelques périodiques européens par Lye M. Yoka. Ces textes très vivants, pleins d'humour ou parfois très profonds, ont un défaut mineur mais quelque peu gênant, celui de ne pas toujours être datés. Ils couvrent les deux dernières décennies du $X X{ }^{e}$ siècle et traitent notamment les événements et les effets de la transition politique entamée en 1990. Dans le deuxième recueil, on trouvera, par exemple, une chronique sur la guerre du 26 août 1998 avec d'intéressantes réflexions sur les formes prises par la violence des habitants contre les envahisseurs et leurs prétendus complices, sur la diabolisation des détenteurs de téléphones portables ou d'appareils satellitaires soupçonnés d'appartenir à une communauté ennemie omniprésente Peut-être y décèlerait-on parfois un peu trop d'indulgence, ou de complaisance, envers les auteurs des violences (Yoka Lye M., 1995, 1999)

Voici une nouvelle contribution à un thème qui a inspiré déjà divers auteurs: la comparaison entre Kinshasa et Brazzaville (Gondola C., 1997).

C'est dans les villes que la musique africaine a évolué le plus fortement. La musique «traditionnelle» a été longtemps mal connue (à part le stéréotype des chants des piroguiers de l'Uele). On s'intéressait davantage à l'ethnographie des instruments de musique. Dans les villes, entre les deux guerres, on assiste à une adaptation des danses et des chants villageois accompagnant certaines céré- monies sociales (comme les deuils et les funérailles). C'est là aussi que les musiciens africains sont en contact avec les chansons venant d'Europe puis, par l'intermédiaire de l'Europe, avec la musique négro-américaine. C'est là aussi que les missionnaires ont cherché à africaniser les chants liturgiques (Romain V., 2001). Sur le développement de la rumba à Léopoldville, pendant l'époque coloniale, on lira l'article de Munda Tehebwa, 2008. La musique moderne congolaise est essentiellement un produit de la ville. A partir de groupes de jeunes musiciens qui ont fait leur apparition à Léopoldville pendant la deuxième guerre mondiale, elle a été le terrain par excellence où l'Africain a pu échapper au patronage européen. Depuis les années 70, il y a eu effacement de la musique brazzavilloise par rapport à la musique kinoise et déplacement d'une partie des espaces de création vers Paris (Strasbourg-St-Denis) et Bruxelles (Matonge). On retiendra aussi des phases d'inhibition locale par les logiques politiques (socialisme scientifique au Congo-Brazzavillle, authenticité au Zaïre) qui ont contraint les musiciens à célébrer les régimes en place, mais aussi une mondialisation de la production ainsi que des phases de renouvellement. La musique congolaise "demeure solidaire de l'évolution sociale et politique» (Gondola C.D., 1993).

La vie au quotidien dans les villes est illustrée de façon naïve mais vigoureuse dans la peinture populaire. Celle-ci commence d'ailleurs à être connue en Europe et en Amérique du Nord (Knockaert A., 2004; Jewsiewicki B., 2002).

Sur divers aspects de la culture urbaine, particulièrement dans le domaine de la littérature et surtout pendant l'époque coloniale, on consultera les ouvrages de (ou édités par) Halen P. (1993). L'un de ceux-ci traite des images de l'Afrique et du Congo dans la littérature des lettres françaises de Belgique.

\section{Lubumbashi}

Lubumbashi a fait l'objet d'études récentes entreprises dans le cadre de son Université en collaboration avec des 
centres de recherches de Belgique et du Canada. Ainsi un projet de recherches élaboré en commun avec des centres de I'Université de Bruxelles et de l'Université de Liège a créé un Observatoire des changements urbains (devenu par la suite un centre de recherches de I'Université de Lubumbashi) qui a dressé des images précises et détaillées de la situation. Plusieurs ouvrages en rendent compte, sous la direction ou sous la plume de Pierre Petit. Voici d'abord, sous sa direction, un premier rapport des recherches faites de juin à octobre 2000 (Petit P., 2001) puis un article qui commente les premiers résultats. Par suite du regroupement de Kasaïens "refoulés» d'autres villes du Katanga et de l'afflux de réfugiés de guerre, la ville aurait atteint 1200000 habitants en 2002. Rappelons qu'en 1994, la population de la ville dont la croissance avait fortement ralenti, était estimée à 750000 habitants (Bruneau J.Cl., 1995). L'analyse d'un échantillon de 84 ménages répartis dans les différents quartiers montre que, par rapport à 1973, il y a augmentation de la taille des ménages: 7,5 personnes en 2000 contre 6,6 en 1973. II y a diversification des ressources budgétaires (63\% des ménages pratiquent au moins une forme de commerce quel que soit leur niveau social). La part du travail salarié s'est fortement réduite. Celle des dépenses alimentaires dans le budget a diminué: $44,6 \%$ en 2000 contre $61,5 \%$ en 1973 , dans un budget total moyen mensuel actuel de l'ordre de 160 US \$ par ménage. Mais c'est grâce à l'accroissement de l'autoproduction vivrière y compris dans les parcelles habitées. L'ingéniosité du vocabulaire de l'économie seconde, dont l'article fournit quelques exemples, traduit bien les difficultés de la vie en ville (Petit P., 2002). Celles-ci sont développées dans un ouvrage publié en 2003, avec le même éditeur, qui, outre de nombreuses informations sur la vie quotidienne, comporte une étude des budgets ménagers permettant une comparaison avec l'enquête de Houyoux J. et Lecanoet Y. publiée en 1975 (Petit P., 2003). Ces difficultés apparaissent aussi très bien dans le domaine de l'alimentation auquel est consacré un autre ouvrage, intitulé Byakula, c'est-à-dire «les aliments» (Petit P., 2004). Sont étudiés ainsi 1) les réseaux d'approvisionnement (aliments de brousse, agriculture périurbaine, calendrier alimentaire, réseaux formels et réseaux informels); 2) la sphère domestique de l'alimentation (repas, préparation, conservation, la journée alimentaire, la part dans les budgets ménagers); 3) les conceptions sur la disette, la malnutrition et la prise en charge de ces problèmes; 4) les réseaux sociaux et les interdits alimentaires; 5) les représentations populaires et les aspects symboliques de l'alimentation.. On verra notamment dans ce dernier et gros chapitre que, si la crise a contraint les Lushois à renoncer à certaines habitudes de consommation et à en adopter d'autres, cela s'est toujours fait en respectant les interdits.

Un autre ouvrage résume les résultats de l'enquête portant sur un dixième des ménages de l'agglomération en juilletaoût 2002, analyse les sources de revenus et évalue la qualité de l'habitat (Nkuku Khonde et Rémon M., 2006).

L'Observatoire sur le changement urbain a dénombré en 2003, 2000 enfants dans la rue et 700 enfants de la rue, Ces derniers dorment dans des abris précaires, les «karemas» (sous un étal de marché, dans une cabine téléphonique, dans un kiosque de magasin). Ces shege sont à la fois, pour les autorités politiques et la police, un instrument informel à manipuler, des victimes à sauver, des êtres insignifiants à bafouer et une force dangereuse à réprimer. «Du fait de la logique d'action des représentants de l'ordre et des enfants de la rue, la tension sous-jacente à leurs rapports est traversée par un courant alternatif, entre collaboration et confrontation» (Kahola Tabu O. et Rubbers B., 2008).

\section{Les relations quotidiennes avec l'Etat.}

La situation peut paraître paradoxale. Alors que l'Etat est défaillant et quasi inexistant et ne fournit plus en tout cas tous les services qu'il doit au citoyen, les fonctionnaires sont omniprésents dans la vie quotidienne: douaniers ou agents du fisc préle- 
vant des taxes officielles ou officieuses, policiers réclamant des amendes pour des délits réels ou imaginaires, employés municipaux percevant une rémunération occulte pour la délivrance de tout document officiel, etc. Ces scènes de la vie courante sont décrites dans les interviews d'acteurs caractéristiques de la vie urbaine: un transporteur (à vélo) de charbon de bois, un boulanger (grec), un marchand de matériaux de construction, un taximan, un tenancier de nganda (lieu de rencontre de type snack-café), une commerçante du marché, un pasteur, des changeurs d'argent, un tenancier d'un cybercafé, etc. (Trefon Th., 2007). Voir aussi sur le secteur informel dans la ville, l'article de Rubbers B. (2007).

La multiplication des ONG locales depuis la fin des années 80 en milieu urbain (auparavant elles étaient mal tolérées par le régime mobutiste) serait une étape vers l'éveil d'une société civile. Le cas est traité à Lubumbashi, en comparaison avec la situation à Cotonou (Pirotte G. et Poncelet M., 2002; Poncelet M. et al., 2006).

Voici quelques contributions mineures à des aspects de la vie sociale et économique Un signe de différenciation sociale a été l'apparition de grandes antennes paraboliques orientables (plus de 2,7 m de diamètre) permettant de capter les émissions de télévision retransmises par satellite. Elles sont possédées par 0,6\% des ménages et sont localisées presque exclusivement dans la «ville» (centre d'affaires et quartiers résidentiels aisés) et sont absentes des quartiers populaires (Lufuma Kapenda A., 2000).

Une recherche intéressante, mais qui n'a pas donné de résultat concluant, a été menée dans un échantillon de 1000 ménages répartis dans 28 quartiers, sur la corrélation entre les caractéristiques de l'environnement et la nature des maladies dont ces ménages ont souffert. Les quartiers ont été classés par l'analyse factorielle puis il a été procédé à une analyse combinée de la matrice de l'environnement et de la matrice des maladies. Aucune corrélation n'a pu être établie, en grande partie sans doute parce que les maladies sont mal identifiées par la population (Assumani S., Kakese B. et Solotshi M., 1993).

\section{LES AUTRES VILLES}

Le petit commerce reste un élément de stabilité sociale. A Kisangani, ville qui compte 330000 habitants au début de la décennie 1990, il emploie 12000 personnes dont une bonne part de façon occasionnelle. II y a 1100 étals dans les rues et 247 boutiques dans les quartiers où les grandes artères sont équipées en électricité. II y a une vingtaine de marchés de jour et 46 marchés nocturnes (alimentaires d'appoint). Les Libanais ont repris la plupart des commerces "zaïrianisés» qui ont fait faillite et tiennent donc le commerce «moderne» (Baya Ki Malanda, 1993).

Un autre exemple de la relation avec l'administration nous est fourni par l'analyse du fonctionnement du cadastre, notamment à Mbuji Mayi. Documents et autorisations ne s'obtiennent pas par des voies directes mais par un système indirect de relations avec les différents membres de l'administration où chacun de ceux-ci perçoit sa part (Piermay J.L., 1993).

La libéralisation de l'exploitation et de la commercialisation du diamant artisanal a accéléré encore la croissance démographique de Mbuji Mayi, qui compterait vers 1995 environ 700000 habitants. Dès 1984, 10000 «diamantaires» y sont recensés officiellement. Cette activité a entraîné le développement d'un réseau parallèle de postes et de télécommunications, version informelle congolaise de $\mathrm{DHL}$, les coursiers-convoyeurs (les «tindeurs») qui transportent le diamant à Kinshasa et en rapportent des valises d'argent-papier. Nombreux hôtels, bars, restaurants. L'aéroport est le deuxième du pays pour le transport des voyageurs. Dans le Kasai occidental, Tshikapa, autre ville du diamant, est aussi en pleine expansion (Nzuzi Lelo, 1995).

Bien que le chef-lieu ait été transféré à Bandundu depuis 1967, Kikwit est resté la ville la plus importante de sa province (200 000 habitants en 1998? les autorités 
communales parlent de 450 000). Et cela malgré l'absence d'industrie, la pénurie d'eau (on puise dans les rivières) et d'électricité (la ville est en permanence dans l'obscurité), les menaces de l'érosion (une quarantaine de ravines déchirent la ville, voir l'article de Salomon, 1998, cité plus haut). C'est que Kikwit bénéficie de sa relative proximité de Kinshasa, par la route, de sa localisation dans une région bien peuplée et à forte activité agricole, d'une population relativement instruite et fortement christianisée (la radio locale est celle de l'évêché). Formée essentiellement de gens venant des régions proches, sa population a conservé de nombreux liens avec le milieu rural et par conséquent continue à être contrôlée, dans une certaine mesure, par les anciens du milieu rural, ce qui se marquerait par l'absence d'enfants de la rue (du moins une bien plus faible proportion qu'à Kinshasa) ou de petits voleurs à la tire. La majeure partie des ménages recourent à l'agriculture pour assurer leur subsistance. Fait remarquable: la ville est à prédominance féminine. Les jeunes hommes sont attirés par les zones minières de l'Angola (où ils travaillent de façon clandestine) ou de la zone frontalière (exploitations de diamant de Tembo, dans le lit du Kwango). Le ménage moyen est plus petit qu'à Kinshasa (6,8 membres contre 7,9). La situation sanitaire est préoccupante. La ville a connu en mars 1995 une épidémie de fièvre Ebola (Ngondo a Pitshandenge, 2000b). Dans la vie économique de la ville, les Bana Lunda ont joué un rôle non négligeable. Mais le bilan social est sans doute assez lourd: déstabilisation des foyers, développement du sida, accroissement du coût de la vie, perte d'une partie scolarisée de la population masculine. Par contre on notera une amélioration de I'habitat, le développement du plurilinguisme (le portugais est parlé dans la ville), des investissements dans de nombreux petits commerces mais aussi dans l'hôtellerie, les bars, etc. et des activités qu'on ne s'attendrait pas à trouver dans une ville intérieure comme des cambistes ou des comptoirs de diamant. Au total, la présence et les activités des Bana Lunda auraient contribué à la résistance à la crise (Sima J., 2001).

Au confluent du Kwango et du Kwilu, Bandundu, cet ancien poste colonial assoupi (il s'appelait Banningville de 1932 à 1966), dont le rang administratif a varié au cours du temps (chef-lieu d'un grand district avant 1932 puis simple chef-lieu de territoire), port en déclin, est devenu, à la suite d'une réorganisation du Zaïre en 1969, le chef-lieu de la grande province, qui porte son nom et qui est constituée de toute la partie orientale de l'ancienne province de Léopoldville. II a reçu ainsi de nouveaux équipements et a vu s'installer de nombreux fonctionnaires. Sa vie politique était dominée par des «barons» du pouvoir Mobutu. On y parle d'ailleurs le lingala. La ville, qui avait 64000 habitants en 1970, en aurait 134000 en 1990. La ville semble s'imposer difficilement dans sa province en raison de l'existence d'une ville plus importante, Kikwit (Maboloko Ngulambangu C.E. et Mbenga Mpiem Ley, 1995).

Qu'est devenue la ville de Kigali, après la catastrophe de 1994? Un rapport établi pour un organisme de l'ONU, indique qu'un an plus tard, la ville a retrouvé le même nombre d'habitants qu'avant le génocide (en 1993, 262 000; en avril 1994, 80000 morts; en juillet 1994, moins de 90000 habitants; en 1995, 270 000) mais la composition a totalement changé. Au cours des massacres, peu d'immeubles ont été détruits. La ville est tenue désormais par des élites tutsi et l'on peut craindre un développement fondé sur des exclusions ethniques et sociales (Pérouse de Montclos M.-A., 2000). Sur les enfants de la rue à Kigali, voir Lenoble-Bart A., 1996. 


\section{LA DIASPORA}

Ecrit d'une plume alerte, pourfendant certaines idées reçues et jetant sur les faits un regard souvent caustique, un livre s'interroge sur les causes de la migration africaine vers l'Europe, sur les problèmes rencontrés dans le pays d'arrivée et sur la contribution que la migration peut apporter au développement du pays de départ. II s'intéresse principalement aux étudiants (pendant longtemps les Congolais de Belgique ont été surtout des étudiants) et aux demandeurs d'asile. La déception devant la difficulté à obtenir un emploi correspondant à ses diplômes et devant l'obligation d'accepter des travaux considérés comme dégradants, transforme le jeune immigré, comme le dit la chanson du musicien congolais Vital, en une «feuille morte». II paraît que le livre lors de sa publication, avait suscité quelque émotion parmi la communauté africaine de Bruxelles, inquiète que l'auteur dévoilât les astuces ingénieuses mais illicites utilisées pour contourner les obstacles que l'administration belge cherchait à opposer aux immigrants ou pour bénéficier d'aides diverses. Dans sa conclusion dans l'ensemble peu optimiste, l'auteur plaide notamment pour le rapatriement au Congo, rapatriement éventuellement assorti d'une amnistie pour les capitaux qui ont été transférés en Europe par les bénéficiaires du régime mobutiste (Mayoyo Bitumba Tipo Tipo, 1995). Sur le même thème, mais avec une perspective plus historique, voir aussi la publication de Zana Aziza Etambala Z.A. (1993), qui avait présenté une thèse à la KUL en 1989 sur "Présences congolaises en Belgique, 1885-1940». Elle décrit l'arrivée de Congolais, depuis les expositions d'Anvers de 1885 et 1894, celle de BruxellesTervuren en 1898, les petits boys, souvent enfants de chefs envoyés par des officiers, des enfants venant vers 1900 pour acquérir une instruction de niveau primaire et devenir des auxiliaires de missions, des jeunes Congolais qui, se trouvant en Belgique, se sont enrôlés dans l'armée belge et ont combattu sur le front en 1914 la création de l'Union Congolaise (Société de Secours et de Développement Intellectuel et Moral de la Race Congolaise!) à Bruxelles, en 1919, l'arrivée aussi de quelques commerçants, et, au milieu des années 50, celle des premiers étudiants universitaires que se disputent Bruxelles et Louvain.

En 1991, il y a un peu plus de 13000 Africains non méditerranéens dans l'agglomération de Bruxelles dont 8000 Congolais, avec quelques noyaux de concentration à «Matonge» (près de la Porte de Namur), dans l'ouest du Pentagone et dans quelques îlots à Laeken. Cette population a un niveau d'instruction généralement élevé, les adultes étant souvent venus pour des études supérieures ou post-universitaires et n'étant pas rentrés au pays, parfois en raison des conditions politiques. Elle est cependant marginalisée dans l'accès à l'emploi (28 \% seulement de personnes actives). L'étude, subsidiée par la Région de Bruxelles-Capitale, analyse les processus de survie, décrit des exemples d'itinéraires et de processus d'intégration et fait quelques propositions, notamment pour que cette population puisse mieux valoriser son savoir dans la société d'accueil (Mashini Dhi Mbita Mulenghe, 1998). Les Congolais sont plus nombreux en France qu'en Belgique (22 740 en 1993 contre 14606 en Belgique). Lututala a mené une enquête qui montre que $14 \%$ seulement des migrants à Paris ont l'intention de retourner définitivement dans leur pays, $12 \%$ sont indécis, tous les autres ont décidé de rester en France. II montre la difficulté à estimer le montant des transferts monétaires, l'intérêt d'enquêter au Congo sur la façon dont les fonds transférés sont utilisés. II établit un tableau de la pertinence et des limites des sources de données que l'on peut recueillir (sources documentaires et enquêtes). II a ressenti la difficulté pour un Congolais, qui continue à résider dans son pays, d'enquêter auprès des Congolais de la diaspora, qui, malgré souvent la précarité de leur propre situation, manifestent fréquemment un sentiment de supériorité envers ceux qui sont restés au pays (Lututala B., 2006). 
Sur le rôle des membres de la diaspora dans les trafics entre l'Europe et le Congo, voir MacGaffey J. et Bazenguissa, 2000.

Un exemple de transferts d'encadrements du Congo à la communauté congolaise installée en Suisse (3 100 personnes envi- ron): dans un groupe de demandeurs d'asile arrivés à partir de 1980, des femmes ont reconstitué des tontines et des mutuelles informelles (likembela et moziki) analogues à celles de Kinshasa (Bagalwa Mapatano J., 2000).

\section{CONCLUSION}

Avec cette chronique, qui couvre une grosse quinzaine d'années et qui est la vingt et unième de sa série, c'est donc soixante années de publications sur le Congo, le Rwanda et le Burundi qui ont été passées en revue, d'abord par Pierre Gourou de 1949 à 1960, par Pierre Gourou et nous-même de 1961 à 1964, par nous-même à partir de 1965.

S'il apparaît difficile et sans grande signification de tenter un bilan de la période couverte par cette chronique, on ne peut s'empêcher de faire quelques constatations.

La figure 1 confirme ce qui a été dit dans l'introduction de cette chronique, c'est-àdire, dans le domaine des sciences humaines, la forte proportion des études menées en milieu urbain (un peu plus de $40 \%$ ) et, pour l'ensemble des recherches, une localisation prédominante sinon exclusive, d'une part au sud-ouest d'une ligne allant du confluent Congo-Kasai au sud du lac Tanganyika et, d'autre part dans les hautes terres orientales (Kivu, Ituri, Burundi, Rwanda).

Dans des pays déchirés par des événements dramatiques, des guerres civiles, des passages de troupes armées diverses, l'effondrement de l'économie et de l'Etat et la crise de l'enseignement et des universités, cette période n'a guère été propice à une recherche scientifique détachée des contingences matérielles. Cela se marque dans les thèmes étudiés. II y a eu pléthore de thèmes politiques traités souvent par des observateurs extérieurs. Mais pour les autres domaines, en dehors de la recherche historique proprement dite où le point de vue africain s'affirme progressivement, il y a eu peu d'orientations nouvelles. On a progressé très modérément dans les domaines habituels. On éprouve, à maintes reprises, l'impression de redites, plusieurs auteurs traitant parfois des mêmes sujets en allant jusqu'à utiliser des titres à peu près identiques. Le domaine rural, très négligé, a été traité souvent à partir de recherches antérieures à 1994. Les études urbaines ont été nettement plus fournies mais sur des thèmes déjà abordés dans la décennie antérieure. Là aussi il y a surabondance de certains sujets comme par exemple les activités du secteur informel qui tend à être considéré désormais comme un élément permanent du système économique et social.

Pour les espaces qui ont été frappés par les événements les plus violents, nous manquons, du moins à notre connaissance, de travaux qui permettraient de faire le point sur les changements qui en ont résulté et finalement sur la façon dont les problèmes se posent aujourd'hui. Tout au plus disposons-nous de témoignages de journalistes. Nous connaissons mal les caractères et les effets des interventions de nouveaux agents extérieurs, comme ceux qui, à la faveur des événements et des changements gouvernementaux, se disputent les anciennes concessions minières. Aucune étude précise n'a été faite jusqu'à présent sur les effets des interventions de la Chine que sa faim de matières premières conduit à s'intéresser de plus en plus au Congo.

Un vaste champ s'ouvre certainement aux chercheurs. Le rétablissement de la paix et l'aboutissement des réconciliations nationales devraient leur permettre de l'exploiter. Mais il est à craindre que ce ne soit pas dans l'immédiat. Au moment d'achever cette chronique, nous avons sous la main un de ces bons ouvrages de vulgarisation et de documentation qui vient d'être publié en 2009 (Huart A. et Tombeur F.). Le titre 
Congo, un pays magnifique, est d'un enthousiasme communicatif, peut-être un peu trop appuyé (le livre est destiné à servir de document de référence pour tout le système éducatif du pays). Son optimisme certes ne manque pas de fondements. Qui pourrait nier en effet les immenses poten- tialités de ce territoire? Mais leur mise en valeur exige, tant dans les campagnes que dans les villes, une évolution des encadrements sociaux et politiques, qui est bien lente à se manifester et qui ne peut être accomplie que par les habitants eux-mêmes.

\section{RÉFÉRENCES BIBLIOGRAPHIQUES}

\section{ABRÉVIATIONS UTILISÉES POUR LES REVUES ET LES ÉDITEURS CITÉS DANS CETTE BIBLIOGRAPHIE}

ARSOM: Académie Royale des Sciences d'Outre-Mer, Bruxelles; Koninklijke Academie voor Overzeese Wetenschappen.

Bull. ARSOM: Bulletin des Séances de I'ARSOM: Mededelingen der Zittingen van de KAOW

Bull. SOBEG: Bulletin de la Société Belge d'Etudes Géographiques. Tijdschrift van de Belgische Vereniging voor Aardrijkskundige Studies.

RBG: Revue Belge de Géographie (Bulletin de la Société Royale Belge de Géographie), jusqu'à l'année 1999 incluse. Belgeo: Revue Belge de Géographie (depuis 2000), Belgisch Tijdschrift voor Geografie, Belgische Zeitschrift für Geographie, Belgian Journal of Geography

GEO-ECO-TROP: Journal International de Géographie et d'Ecologie Tropicales, édité à Liège. Depuis 2006, la géologie est aussi mentionnée dans le titre.

MRCA: Musée royal de l'Afrique centrale, Tervuren.

Lorsqu'un article comprend plusieurs auteurs, les noms du deuxième et du troisième sont repris séparément avec un renvoi au nom du premier.

Pour certains auteurs congolais, nous n'avons pu établir avec certitude leur nom principal. En cas d'erreur de notre part, nous prions les auteurs de nous excuser.
- ACHARD F., voir MALINGREAU J.-P. (1998).

- Africa's Great Rift: Diversity and Unity, voir DE DAPPER M. \& DE LAME D. (2006).

- AFRICA-TERVUREN, MRCA (2008), Carte administrative et routière de la République Démocratique du Congo, échelle: 1/ 2500000.

- ALEXANDRE J. (2002), Les cuirasses latéritiques et autres formations ferrugineuses tropicales. Exemple du Haut Katanga méridional, MRCA, Tervuren, Sciences Géologiques, Annales, série in$8^{\circ}, 107,118 \mathrm{p}$.

- ALEXANDRE J., ALONI K., DE DAPPER M. (1994), «Géomorphologie et variations climatiques au Quaternaire en Afrique centrale», GEO-ECO-TROP, 16, 1-4; Colloquium Climatic Changes and Geomorphology in Tropical Environments (Brussels, May 1992), ARSOM, pp. 167205
- ALEXANDRE J., voir DIBANGA B. (1996); DEMAREE G. (1998).

- ALI EMEDI A. (2004), «Les PMEA de production et la création d'emploi dans la commune de Kalamu», Vivre et survivre à Kinshasa. Problématique du développement humain, pp. 127-150.

- ALONI K., voir ALEXANDRE J. (1994).

- AMAT-ROZE J.-M. (1995), «Entre développement et sous-développement, l'infection à $\mathrm{VIH}$ et le SIDA en Afrique subsaharienne, dans SIDA et développement en Afrique, Bull. ARSOM, 41, supplément n¹, pp. 79-88.

- AMAT-ROZE J.-M. (2001), «L'infection $\mathrm{VIH} /$ Sida en Afrique sudsaharienne, bilan géographique de deux décennies épidémiques, Bull. ARSOM, 47, 2, pp. 11128.

- AMBE G.A., DELCARTE E \& MALAISSE F. (1999), «Les lianes à eau, providence du villageois assoiffé en savanes 


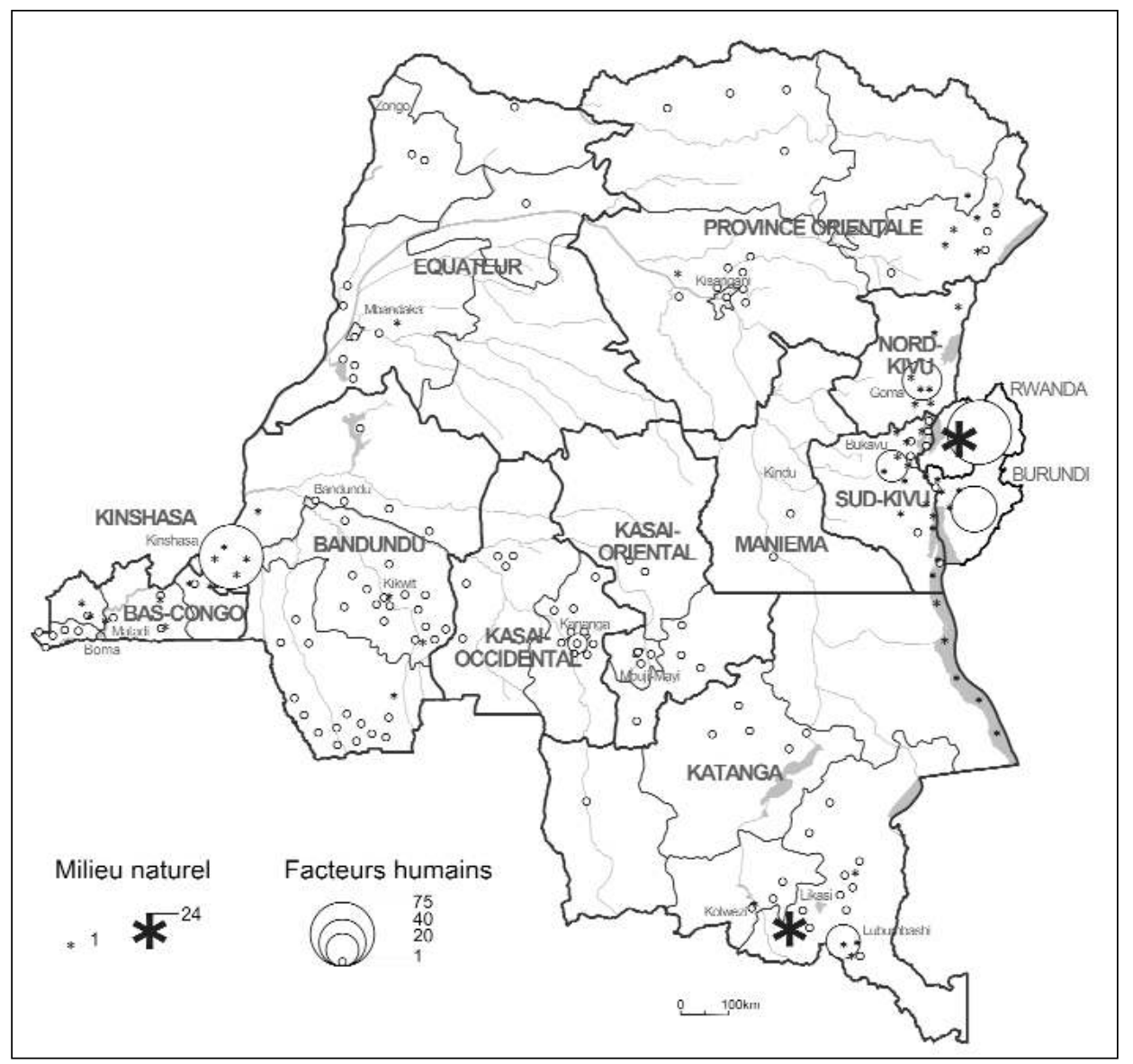

Figure 1. Localisation des références bibliographiques. Les références qui concernent l'ensemble du pays ne sont pas reprises sauf pour le Burundi et le Rwanda.

Fond de carte: E. Wolff. Infographie: Q. Rombaux

soudano-guinéennes: étude quantitative et qualitative de la sève de Cissus populnea Guill. Perr (Vitacae)», GEO-ECOTROP, 23, pp. 107-121.

- AMELOT X. (1997), «Pression démographique et dynamique des systèmes ruraux au Rwanda», Les Cahiers d'OutreMer, 197, pp. 7-26.

- ANDRÉ C. \& PLATTEAU J.-P. (1997), «Land relations under unbearable stress: Rwanda caught in the Malthusian trap", L'Afrique subsaharienne: les perspectives en matière de sécurité, Colloque 2 et 3 décembre 1997, Institut Supérieur de
Défense, pp. 57-107

- ANTHOINE C. (1996), «Le forgeron qui devint roi. Idéologie politique de la chefferie songye des Kalebwe (Zaïre)", Civilisations, 43, 2, pp. 15-44.

- ARBYN M., DEDEURWAERDER M., MIAKALA M., BIKANGI N., BOELAERT M. (1995), «Surveillance de l'état nutritionnel de la population de Kinshasa, Zaïre (1991-1994)», Annales de la Société belge de Médecine tropicale, 75, pp. 115124.

- ARNAUT K. \& VANHEE H. (2001), «De Postkoloniale Afrikanistiek in België en de 
Verenigde Staten. Een interview met Jan Vansina", BVA-ABA FORUM, 21, pp. 1119.

- ARNOULD R. (2005), «La canalisation du fleuve Congo en aval de Kinshasa: un défi pour le XXIe siècle», Bulletin de la Société Géographique de Liège, 46, pp. 99-117.

- ASIDI N., voir KARCH S. (1993).

- ASSANI A. A. (1993), "Etude statistique du nombre de jours secs et de la longueur des périodes sèches au cours de la saison des pluies à Lubumbashi», GEO-ECO-TROP, 17, 1-4, pp.1-14.

- ASSANI A.A. (1994), "Subdivision et caractérisation objectives des saisons au Zaïre au moyen de l'analyse en composantes principales», Bull. SOBEG, LXIII, 2, pp.251-269.

- ASSANI A.A. (1999 a), «Variation temporelle et persistance des épisodes secs en saison des pluies à Lubumbashi», Sécheresse, 10, pp. 45-53.

- ASSANI A. A. (1999 b), «Analyse de la variabilité temporelle des précipitations (1916-1996) à Lubumbashi (CongoKinshasa) en relation avec certains indicateurs de la circulation atmosphérique (oscillation australe) et océanique (El Niño, La Niña)», Sécheresse, 10, pp. 245252.

- ASSANI A. (2001), «Evolution des précipitations dans l'arc cuprifère congolais durant la seconde moitié du XXe siècle», GEO-ECO-TROP, 25, 1-4, pp. 1-16

- ASSANI A. A., BATUBENGA K., N'TUMBA K., SOLOTSHI M. (1993), "Le commerce des huiles végétales sur les marchés de Lubumbashi», RBG, 117, 4, pp. 147-155.

- ASSANI A. \& KALOMBO K. (1995), «Analyse statistique des maximums annuels de pluie journalière à Kolwezi (Zaïre)», GEO ECO-TROP, 19, 1-4, pp. 115.

- ASSANI A. A. \& KALOMBO K. (1997), «Le climat du Bas-Congo (CongoKinshasa). Mise au point sur les facteurs explicatifs", Bull. SOBEG, LXV, 1, pp. 121-131.

- ASSANI A.A., voir DEMAREE G. (1998); KALOMBO (1996, 1999-2000).

- ASSUMANI S., KAKESE B. \& SOLOTSHI M. (1993), "Santé et dégradation d'un environnement urbain de l'Afrique tropicale: le cas de la ville de Lubumbashi (Zaïre)», GEO-ECO-TROP, 17, 1-4, pp. 171-189.

- BAERT G. \& VAN RANST E. (1998), «Importance of Organic Matter for Sustainability of Agro-Ecosystems in Lower Zaire», in DEMARÉE G., ALEXANDRE J., DE DAPPER M. (guest eds.), International Conference on Tropical Climatology, Meteorology and Hydrology. In Memoriam Franz Bultot, Brussels 22-24 May 1996, pp. 730-744.

- BAERT G., voir BOECKX, P. (2006).

- BAgALWA MAPATANO J. (2000), "L'usage féminin des tontines. La "maison congolaise" en Suisse", in MONNIER L. \& DROZ I. (dir.), Côté jardin, côté cour: l'anthropologie de la maison africaine, Paris, P.U.F., pp. 181-207.

- BAGALWA-MAFATANO J., MONNIER L., (2002), "Les sociétés de transfert de fonds entre la Suisse et la République démocratique du Congo", Autrepart (IRD), 22, pp.121-146.

- BAGALWA R., voir MAVANGA T. (1999).

- BAKAJIKA BANKAJINIKA (1997), Epuration ethnique en Afrique. Les «Kasaiens» (Katanga 1961-Shaba 1992), Paris, L'Harmattan, Coll. Eudes africaines, 244 p.

- BAKER A.J.M., voir MALAISSE F. (1997).

- BALENCIE J.-M., DE LA GRANGE A. (dir.) (1999), Mondes rebelles. Guerres civiles et violences politiques, Paris, Michalon.

- BAMBILA MULUMBA H., (2004), «Pauvreté féminine et sécurité alimentaire des ménages en RDC», Vivre et survivre à Kinshasa. Problématique du développement humain, pp. 105-126.

BANGAMWAMBO F.X. et al. (1991), Les relations interethniques au Rwanda à la lumière de l'agression d'octobre 1990: genèse, soubassements et perspectives, Ruhengeri, Editions Universitaires du Rwanda, 352 p.

- BARAMBIRWA J.-B. (1993), «Contraintes hydrobiologiques de mise en valeur du sud-est du Rwanda», L'eau et l'aménagement dans l'Afrique des Grands Lacs, Colloque Bujumbura, 1991, Talence, CRET, pp. 3-19.

- BART F. (avec la coll. de BART A.) (1993), Montagnes d'Afrique, terres 
paysannes. Le cas du Rwanda, Coll. «Espaces Tropicaux», 7, Talence, Centre d'Etudes de Géographie Tropicale, Presses Universitaires de Bordeaux, $588 \mathrm{p.}$

- BART F. (1994a), «La place de la caféiculture de montagne en Afrique», in TULET J-Ch. et al. (dir.), Paysanneries du café des hautes terres tropicales. Afrique et Amérique latine, Paris, Karthala, pp. 48-51.

- BART F. (1994b), «Le café au Rwanda en marge de l'économie vivrière", in TULET J-Ch. et al. (dir.), Paysanneries du café des hautes terres tropicales. Afrique et Amérique latine, Paris, Karthala, pp. 120-144.

- BART F. (2000), «A propos de Pierre Gourou (1953), La densité de la population au Ruanda-Urundi», in NICOLAÏ. H., PÉLISSIER P. \& RAISON J.-P (dir.), Un géographe dans son siècle. Actualité de Pierre Gourou, Paris, Karthala, Géotropiques, pp.123-127.

- BART A. \& BART F., voir IMBS F. (1994).

- BARUTWANAYO M., voir VAN BORTEL W. (1996).

- BATUBENGA K., voir ASSANI A. A. (1993).

- BAYA KI MALANDA (1993), «Le petit commerce à Kisangani», Bulletin Géographique de Kinshasa, GEOKIN, IV, 1, pp. 7-23.

- BAYA KI MALANDA (1999), «Les modes d'approvisionnement en produits vivriers de Kisangani (Zaïre)», Les Cahiers d'Outre-Mer, 207, pp. 323-331.

- BAZENGUISSA R.(2004), «Les réfugiés et les enjeux locaux au nord-est du Congo", in GUICHAOUA A. (dir.), Exilés réfugiés, déplacés en Afrique centrale et orientale, Paris, Karthala, pp. 379-423.

- BAZENGUISSA-GANGA R., voir MAC GAFFEY J. (2000).

- BEGHIN Y., voir TELLIER V. (1996).

- BEKE D. (1996), «Droit foncier informel: un troisième système juridique à côté du droit traditionnel et du droit formel moderne», in DE VILLERS G. (dir.), Phénomènes informels et dynamiques culturelles en Afrique, Cahiers Africains, Afrika Studies, 19-20, pp. 25-32.

- BEKE D. (2000), «Jef Van Bilsen, de onafhankelijkheid van Congo et de visie op Lumumba», Afrika Focus, 16, 1-2, pp. 35-60.

- BEKAERT S. (1996), "Olifant-transformatie in Zaïre: een Sakata genezingsritueel ethnomethodologisch bekeken», Medische Antropologie, Amsterdam, pp. 278-305.

- BELEPE BOPE MABINTCH (2004), «La statuaire royale kuba», in MABIALA MANTUBA-NGOMA P. (dir.), La Nouvelle Histoire du Congo. Mélanges eurafricains offerts à Frans Bontinck, C.I.C.M., Cahiers Africains, 65-66-67, pp. 73-90.

- BENATAR J. \& PIMIENTA-BENATAR M. (2000), De Rhodes à Elisabethville: l'odyssée d'une communauté Séphardie, Paris, Ed. SHAC.

- BERGONZINI L. (1995), «Les écoulements de la Lukuga à l'exutoire du lac Tanganyika», Rapport annuel 1993-1994 du Département de Géologie et de Minéralogie, MRCA, Tervuren, pp. 79-89.

- BERGONZINI L. (1998a), «Les fluctuations du niveau du lac Tanganyika et les termes de référence de son bilan hydrique», in DEMARÉE $G$. et al., International Conference on Tropical Climatology, Metorology and Hydrology, In Memoriam Franz Bultot, Brussels, May 1996, ARSOM, pp. 613-637.

- BERGONZINI L. (1998b), Bilans hydriques des lacs (Kivu, Tanganyika, Rukwa et Nyassa) du Rift est-africain, Tervuren, MRCA, Annales, Sciences géologiques, 103, $183 \mathrm{p}$.

- BERGONZINI L. \& RICHARD Y. (2004), «Relationship between the Zonal Circulation over the Equatorial Indian and Pacific Oceans and the East African Lakes: Victoria, Tanganyika and NyassaMalawi Level Fluctuations», in DEMARÉE et al., Second International Conference on Tropical Climatology, Meteorology and Hydrology, ARSOM, pp. 41-60.

- BERTRAND J. (1997), «Les précédents ougandais», Du Zaïre au Congo, Afrique contemporaine, 183, pp. 31-43.

- BIAYA TSHIKALA K. (2000), «Jeunes et cultures de la rue en Afrique urbaine (Addis Ababa, Dakar et Kinshasa)", Politique africaine, 80, pp. 12-31.

- BIAYA TSHIKALA K. (2005), "Youth Street Culture in Urban Africa. Addis Ababa, Dakar and Kinshasa», in HON- 
WANA A. \& DE BOECK F. (eds.), Makers \& Breakers. Children \& Youth in Postcolonial Africa, Oxford, James Currey, Trenton, Africa World Press, Dakar, Codresria, pp. 215-228.

- BIDINKAM KUPA NGANDU \& BILOWA BIDIKU (1993), "Culture et usages actuels dans la Collectivité de Kafuba au Kasaï-Occidental», Zaïre-Afrique, 271, pp. 42-48.

- BIDOU J.E. (1993), «Riziculture et aménagement des marais dans le Buyenzi», L'eau et l'aménagement dans l'Afrique des Grands Lacs, Colloque Bujumbura 1991, pp. 104-127

- BIDOU J-P. (1994a), «Densités de population, occupation du sol et fertilité dans le Buyogoma», in GAHAMA J. \& THIBON C. (dir.) (1994), Les régions orientales du Burundi. Une périphérie à l'épreuve du développement, Paris, Karthala, pp. 113-135.

- BIDOU J.-P. (1994b), «Exploitations agricoles au Kirimiro et au Buyogoma. Un cycle de vie?», in GAHAMA J. \& THIBON C. (dir.) (1994), Les régions orientales du Burundi. Une périphérie à l'épreuve du développement, Paris, Karthala, pp. 292315.

- BIDOU J. E. (1994c), «Burundi: l'engrenage caféier», in TULET J.-Ch. et al., (dir.), Paysanneries du café des hautes terres tropicales. Afrique et Amérique latine, Paris, Karthala, pp. 146-176.

- BILUSA BAILA BOINGAOLI (1993), «Recherche sur l'identité ethnique du peuple Topoke (Haut-Zaïre). Contribution à I'histoire socio-culturelle du Zaïre», Mélanges Pierre Salmon, II, Histoire et ethnologie africaines, édités par THOVERON G. \& LEGROS H., Civilisations, $X L I$, 1-2, pp. 103-116.

- BIREMBANO BAHATI, voir SEBAKUNZI NTIBIBUKA (1993).

- BIZIMANA B.L., LUMBU B.M., LUKANDA M., MANDUNGU K., CIBAMBULA M. \& MVUEMBA F. (1993), «Evolution sédimentologique du canal d'amenée du barrage hydroélectrique d'Inga (Zaïre)», GEO-ECO-TROP, 17, 1-4, pp. 15-19.

- BIZIMANZ M., voir SIMONARD T. (1994).

- BLANCHART G., DE DEURWAERDE J., NÈVE G., ROBEYNS M. \& VAN BOST P.
(1993), Le rail au Congo belge, tome 1, 1890-1920, Bruxelles, Blanchart et Cie, 400 p.; (1999), tome 2, 1920-1945, 433 p., BLANCHART G. (2008), tome 3, 19451960, Drogenbos, Eder, $432 \mathrm{p}$.

- BLONDEEL W., FOUTRY F., VAN MENSEL F. (2000), «Kongo, schoolvoorbeeld van kolonialisme: een gesprek op 30 juni 1980 van W.Blondeel, V. Foutry en F. Van Mensel met prof. dr. A.A.J. van Bilsen (RUG)», Afrika Focus, 16, 1-2, pp. 1-33.

- BOBB S. (1999), Historical Dictionary of Democratic Republic of the Congo (Zaire), Revised edition, African Historical Dictionaries, 76, The Scarecrow Press, $598 \mathrm{p}$.

- BOECKX P., VAN CleEMPUT O., BAERT G., MAFUKA P. \& TABA N. (2006), "Improvement of Soil Fertility in the Region of Kinshasa (DR Congo) by Using Readily Available Organic Matter », in DE DAPPER M. \& DE LAME D. (eds.), Africa's Great Rift: Diversity and Unity, AfricaTervuren, ARSOM, pp. 340-341.

- BOFEKO ETAKA (2004), «Les Rwandais à Bamanya en 1997", Annales Aequatoria, 25, pp. 536-547.

- BOMBOKO F., voir BOUVIER P. (2004).

- BONNEFILLE R., SCHWARTZ D. \& GDR ECOFIT (2002), «Histoire récente des forêts humides de l'Afrique centrale: de l'Holocène moyen à actuel, perspectives pour l'avenir», Paris, Historiens et Géographes, 37, pp. 225-285.

- BONTINCK F. (1988), Les Missionnaires de Scheut au Zaïre: 1888-1988, Kinshasa, Maison Provinciale C.I.C.M, 72 p.

- BONTINCK F. (1995 a), "Les chameaux du Pool», Zaïre-Afrique, 293, pp. 179-186. - BONTINCK F. (1995 b), «Les Mindele, porteurs d'étoffes», Annales Aequatoria, 16, pp. 135-152.

- BOSCO MUCHUKIWA (2006), Territoires ethniques et territoires étatiques. Pouvoirs locaux et conflits interethniques au Sud-Kivu (R.D. Congo), Anvers, Centre d'Etude de la Région des Grands Lacs d'Afrique, Paris, L'Harmattan, 213 p. - BOSKI T., voir KASEBA (1997).

- BOSMANS M., voir MICHIELSEN K. (2008).

- BOURDEAUX Q., BUYCK B., MALAISSE F., MATERA J., MARLIER M., WATHELET 
B. \& LOGNAY G. (2003), «Wild edible mushrooms from a Zambezian Woodland area (Copperbelt Province, Zambia)», GEO-ECO-TROP, 27, 1-2, pp. 33-44.

- BOURDOUX P., MAHANGAIKO LEMBO E., DE CLIPPELE C., BERTOCCHI I. \& FELEIMA MOHERESSE P. (2005), “Correction de la carence en iode en Afrique centrale», Bull. ARSOM, 51, 4, pp. 469504.

- Bourla eRRERA M. (2000), Moïse Levy. Un rabbin au Congo (1937-1991), Bruxelles, La Longue Vie.

- BOUTTIAUX A.-M. (2007), Afrique: musées et patrimoines pour quels publics?, Paris, Karthala, $176 \mathrm{p}$.

- BOUVIER P. \& IVOR M. (1997), "Institutions et pouvoir au Rwanda et au Burundi», in VERHASSELT Y. (éd.), Rwanda-Burundi. Journée d'étude, Bruxelles, ARSOM, pp. 127-149.

- BOUVIER P. (en coll. avec BOMBOKO F.) (2004), Le dialogue intercongolais: anatomie d'une négociation à la lisière du chaos. Contribution à la théorie de la négociation, Cahiers Africains, 63-64, Tervuren, MRCA, Paris, L'Harmattan, $326 \mathrm{p}$.

- BRAECKMAN C. (1994), Rwanda. Histoire d'un génocide, Paris, Fayard, 341 p. - BRAECKMAN C., CROS M.-F., DE VILLERS G., FRANÇOIS F., REYNTJENS F., RYCKMANS F. \& WILLAME J.-C. (1998), Kabila prend le pouvoir, Les prémices d'une chute. La campagne victorieuse de l'AFDL, Le Congo d'aujourd'hui, Bruxelles, GRIP, Editions Complexe, $180 \mathrm{p}$

- BRANCHU P. (2001), Cycle des éléments majeurs et traces dans les grands lacs de rifts tropicaux (lacs Tanganyika et Malawi). Processus et enregistrements biogéochimiques, MRCA, Tervuren, Annales, Sciences géologiques, 106, $374 \mathrm{p}$.

- BRASSEUR D., GOYENS P. \& VIS H. (1998), «L'intolérance au lactose et la malnutrition protéino-énergétique chez l'enfant dans la région des Grands Lacs», Bull. ARSOM, 44, 4, pp. 619-632.

- BRUGAILLÈRE M.-C. (1993), «Un journal au service d'une conquête: le Mouvement géographique (1884-1908)», in HALEN P. \& RIEZ J. (éd.), Images de l'Afrique et du Congo/Zaïre dans les let- tres françaises de Belgique et alentours, Actes du colloque interuniversitaire de Louvain-la-Neuve, (4-6 février 1993), Textyles, Bruxelles, pp. 20-35.

- BRUNEAU J.-Cl. (1995a), "Crise et déclin de la croissance des villes au Zaïre. Une image actualisée», RBG, 119, 1-2, pp. 103-114.

- BRUNEAU J.-Cl. (1995b), "Crise et déclin de la croissance des villes au Zaïre», Revue de géographie du Cameroun, 12, 1, pp. 15-23.

- BRUNEEL H., VANDEN EEKHOUT A., MOLISHO D., BURKE J., DEGROOF D. \& PÉPIN J. (1994), «Contrôle de la trypanosomiase à T. b. gambiense: évaluation d'une stratégie basée sur le traitement des suspects sérologiques par un don unique de diminazène», Annales de la Société belge de Médecine tropicale, 74, 3, pp. 203-215.

- Bruxelles et le Congo, LAURO A. (directrice scientifique) (2008), Les Cahiers de la Fonderie, Revue d'histoire sociale et industrielle de la région bruxelloise, 38, juin 2008.

- BUELENS F. (2007), Een financieeleconomische geschiedenis, EPO, $671 \mathrm{p}$.

- BUKOME ITONGWA D. \& KINGOMA MUNGANGA J.-P. (2002), «Connectivité et accessibilité du réseau routier de la République démocratique du Congo", Bull. Société géographique de Liège, 42, pp. 61-75.

- BULKENS A. (1998), «At men dan echt uit kalebassen? Proto-bantoe en de domesticatie van cucurbitaceae», Afrika Focus, 14, 1, pp. 67-84

- BULTOT F. \& GELLENS D. (1994), Sur le caractère stationnaire et cyclique des précipitations au Rwanda, Bruxelles, ARSOM, Classe des sciences techniques, Mémoires in- $8^{\circ}$, Nlle Série, 19, 1 , $53 \mathrm{p}$.

- BUSHABU MBENGELE-MING (1998), «Tout développement régional dans le tiers-monde implique la prise en compte des spécificités sous-régionales et des programmes d'actions intégrées: le cas du Katanga (Congo)», Bull. SOBEG, pp. 91-102.

- BUSHABU M.-M., MABIRA M. \& KAPEND M. (2002), «Les besoins énergétiques des ménages de Kananga (RDC)», 
Bull. Société géographique de Liège, 42, pp. 53-60.

- BUSHOKI BATABIHA J. (1994), «La dissolution de la caféiculture au Kivu (Zaïre)», in TULET J.-Ch. et al. (dir.), Paysanneries du café des hautes terres tropicales. Afrique et Amérique latine, Coll. «Hommes et Sociétés», Paris, Karthala, pp. 178-191.

- CAMBRÉZY L. (1997), «Un aspect méconnu de la crise rwandaise, les réfugiés de Nairobi», Politique africaine, décembre 1997.

- CAMBRÉZY L. (1998), «Une enquête chez des réfugiés urbains: le cas des exilés rwandais à Nairobi», in LASSAILLYJACOB V. (éd. scientifique), Communautés déracinées dans les pays du Sud, Autrepart, Cahiers des Sciences humaines, nelle série, $\mathrm{n}^{\circ}$ 5, ORSTOM, pp. 79-94.

- CASTRYCK G. (2007), "Moslims in Usumbura (1897-62). Sociale geschiedenis van de islamitische gemeenschappen van Usumbura in de koloniale tijd », Revue Belge d'Histoire contemporaine, XXXVII, 1-2, pp. 257-266.

- CAZENAVE-PIARROT A. (2004), «Burundi: une agriculture à l'épreuve de la guerre civile», Les Cahiers d'OutreMer, 226-227, pp. 313-338.

- CAZENAVE-PIARROT A. (2007), «Ruptures et recompositions rurales en Afrique des Grands Lacs», in VENNETIER P. (dir.), A propos de développement rural en Afrique Noire, coll. Espaces enclavés, 1, IOM, ADES, DYMSET, pp.7-36.

- CEYSSENS R. (2007), «Urbanisme et vision de la ville dans les royaumes de la savane», in VELLUT J.-L. (dir.), Villes d'Afrique. Explorations en histoire urbaine, Cahiers Africains, 73, pp. 25-40.

- CHARLERY de la MASSELIÈRE C. (1994), «Versants du Rwanda: la gestion de l'incertitude», Bordeaux, Les Cahiers d'Outre-Mer, 185, pp.7-22.

- CHARLET J.-M. (guest editor) (1997), Colloque international Cornet. Centenaire des premières études sur la géologie shabienne (Zaïre), "Gisements stratiformes de cuivre et minéralisations associées", 5-9 septembre 1994, Mons, Faculté polytechnique de Mons et ARSOM, $482 \mathrm{p}$.
- CHARLET Ch. \& LORIS H. (2005), «The Uraniferous Metallogenic Mineralization of the Katanga-Zambia Province», Bull. ARSOM, 51, 4, pp. 507-538.

- CHARLET J.-M. \& MAKABU G. (2000), «La prospection des gisements cachés d'uranium et métaux associés par les techniques gaz, le cas du Katanga», Bull. ARSOM, 46, 3, pp. 319-338.

- CHARLIER J. (1993), «La Voie nationale du Zaïre», Bull. ARSOM, 39, 2, pp. 271276.

- CHARLIER J. (1995), «La lutte contre la jacinthe d'eau au Congo-Zaïre», Bull. ARSOM, 41, 2, pp. 225-233.

- CHARLIER J. (1998), «Pourra-t-on sauver les connaissances hydrologiques du bassin congolais/zaïrois?», in DEMARÉE G. et al., International Conference on Tropical Climatology, Meteorology and Hydrology, In Memoriam Franz Bultot, Brussels, May 1996, ARSOM, pp. 492-496.

- CHARLIER J. (1997), «Les problèmes du désenclavement du Rwanda et du Burundi», in VERHASSELT Y. (éd.), Rwanda-Burundi. Journée d'étude, Bruxelles, 10 janvier 1997, ARSOM, pp. 87-110.

- CHARLIER J., M'PENE Z. (2006), «L'axe ferroviaire Matadi-Kinshasa, clé de voûte du système des transports de la République Démocratique du Congo», Le chemin de fer en Afrique, Paris, KarthalaPRODIG-SEDET, pp. 147-159.

- CHARTRY G., voir TACK L. (2006).

- CHRÉTIEN J.-P. (1993), Burundi: I'histoire retrouvée. 25 ans de métier d'historien en Afrique, Paris, Karthala, $509 \mathrm{p}$.

- CHRÉTIEN J.-P. (1995), Rwanda: les médias du génocide, Paris, Karthala, 397 p.

- CHRÉTIEN J.-P. (1997), Les défis de l'ethnisme: Rwanda et Burundi: 19901996, Paris, Karthala, 400 p.

- CHRÉTIEN J.-P. (2000), L'Afrique des Grands Lacs: deux mille ans d'histoire, Paris, Aubier, $411 \mathrm{p}$.

- CHRÉTIEN J.-P. (2003), L'Afrique des Grands Lacs: deux mille ans d'histoire, Paris, Flammarion, coll. Champs, $411 \mathrm{p}$.

- CHRÉTIEN J.-P. (2004), «Les capitales royales de l'Afrique des Grands Lacs peuvent-elles être considérées comme des villes?», Journal des africanistes, 74, 
1-2, pp. 277-298.

- CHRÉTIEN J.-P. (2006), «La face cachée de la région des Grands Lacs: l'histoire des vallées et des lacs", International Conference "Africa's Great Rift. Diversity and Unity», Royal Academy for Overseas Sciences, Royal Museum for Central Africa, Brussels, 29-30 September 2005, pp. 113-135.

- CHRÉTIEN J.-P. \& DUPAQUIER J.-F. (2007), Burundi 1972, au bord des génocides, Paris, Karthala, 494 p.

- CHRÉTIEN J.-C. \& GAHAMA J. (1995), «Les options d'une indépendance sous tutelle au Rwanda et au Burundi», in AGERON C.R. \& MICHEL M. (dir.), L'ère des décolonisations, sélection de textes du colloque "décolonisations comparées», Aix-en-Provence, 30 septembre3 octobre 1993, pp. 22-238.

- CHRÉTIEN J.-P. \& MUKURI M. (dir.) (2002), Burundi, la fracture identitaire: Logiques de violence et certitudes ethniques, 1993-1996, Paris, Karthala, 468 p. - CHRÉTIEN J.-P. \& PRUNIER G. (dir.) (2003), Les ethnies ont une histoire, $2^{\mathrm{e}}$ édition (la première est de 1989), Paris, Karthala, $435 \mathrm{p}$.

- CIBAMBUlA M., voir BIZIMANA B.L. (1993).

- CiRABA M., voir MUNyOlOlO Y. (1994).

- Conférence sur la Territoriale (1998), Kinshasa, 12-14 février 1998, CongoAfrique, 324, pp. 213-237.

- COCHET H. (1996), «Gestion paysanne de la biomasse et développement durable au Burundi», ORSTOM, Cahiers des Sciences humaines, 32, 1, pp. 133151.

- COCHET H. (2001), Crises et révolutions agricoles au Burundi, Paris, INAPGKarthala, $468 \mathrm{p}$.

- COCHET H. (2000), «Diversité et modernité des paysages agraires du Burundi», in NICOLAÏ H., PÉLISSIER P. \& RAISON J.-P (dir.) (2000), Un géographe dans son siècle. Actualité de Pierre Gourou, Paris, Karthala, Géotropiques, pp. 93-107.

- COCHET H. (2001), "Crises et révolutions agricoles au Burundi: les leçons de l'histoire», Table Ronde, 27-11-1998, Collection Espaces tropicaux, Pessac,
Dymset, 16, pp. 161-177.

- CODERE H. (1993), «Perception of caste in Rwanda 1959-1960», Liber Amicorum Marcel d'Hertefelt, Essais anthropologiques (sous la dir. de P. WYMEERSCH), Cahiers Africains, Afrika Studies, 4-5, Bruxelles, Institut AfricainCEDAF, pp. 93-113.

- COOSEMANS M., voir VAN BORTEL W. (1996).

- CORNELIS S. (2008), «Culture coloniale, acteurs culturels et arts visuels au Congo (1920-1950)», in QUAGHEBEUR M. et BIBIANE TSHIBIOLA (dir.), Aspects de la culture à l'époque coloniale en Afrique centrale, 9, pp.145-170.

- CORNELISSEN E. (2002), «Human Responses to Changing Environment in Central Africa between 40000 and 12000 B.P.», Journal of World Prehistory, 16, pp. 197-236.

- CORNET A. (1995), «Famine noire et regards blancs: la famine Rwakayikura dans le Rwanda des années 20», in DEVISCH R., DE BOECK F., JONCKERS D. (dir.) (1995), Alimentation, traditions et développement en Afrique intertropicale, Paris, L'Harmattan, pp. 180-203.

- CORnet A. (1996), Histoire d'une famine, Rwanda 1927-1930: crise alimentaire entre tradition et modernité, Louvainla-Neuve, UCL, Centre d'histoire de l'Afrique, $156 \mathrm{p}$.

- CORNET A. (2005), Action sanitaire et contrôle social au Rwanda (1920-1940). Femmes, missions et politiques de santé, thèse UCL, 5 volumes.

- CORNET A. \& BONTINCK F. (éd.) (2001), Emeri Cambier: correspondance au Congo (1888-1899); un apprentissage missionnaire, Institut Belge de Rome, Bruxelles, $478 \mathrm{p}$.

- CROMBOIS J.-F. (1998), «Le Congo et l'ethnographie: colonialisme, sociologie et ethnologie en Belgique avant 1914", Civilisations, $X L V, 1-2$, pp. 55-84.

- CROS M.-F. \& MISSER F. (2006), Géopolitique du Congo (RDC), Collection Géopolitique des États du monde, Bruxelles, Editions Complexe, 143 p.

- DALEY P. (2006), «Ethnicity and Political Violence in Africa: the Challenge to the Burundi State», Political Review, 25, 6, pp. 657-679. 
- DARCIS L. (2007), «Les Belbase: une réalisation peu connue de l'expansion belge en Afrique de l'Est», Bull. ARSOM, 53, 2, pp. 131-146.

- DE BOECK F. (1993), «Symbolic and diachronic study of inter-cultural therapeutic and divinatory roles among aLuund (Lunda) and Chokwe in the Upper Kwaango (South Western Zaire)», Afrika Focus, 9, 1-2, pp. 73-104.

- DE BOECK F. (1994), «When hunger goes around the land: hunger, famine and the catalysing of societal and cultural order in Luunda land", Man, 28, pp. 1-25. - DE BOECK F. (1995), «"Lorsque la faim court le pays": la faim et l'alimentation chez les Luunda du Zaïre», in DEVISCH R., DE BOECK F., JONCKERS D. (dir.) (1995), Alimentation, traditions et développement en Afrique intertropicale. Paris, L'Harmattan, pp. 85-115.

- DE BOECK F. (1998a), «Identité, résistance et 'effervescence' sociale: perspectives locales et globales au Zaïre», in DE VILLERS G. (dir.), Phénomènes informel et dynamiques culturelles en Afrique, Cahiers africains, Afrika Studies, 19-20, pp. 184-218.

- DE BOECK F. (1998b), «Au-delà du tombeau: histoire, mémoire et mort dans le Congo/Zaïre postcolonial», in GROOTAERS J.-L. (dir.), Mort et maladie au Zaïre, Cahiers Africains, 31-32, pp. 129-172.

- DE BOECK F. (1998c), «Domesticating Diamonds and Dollars: Identity, Expenditure and Sharing in Southwestern Zaire (1984-1997)», Development and Change, 29, 4, pp. 777-810.

- DE BOECK F. (1999), «Dogs breaking their Leash. Globalisation and Shifting Gender categories in the Diamond Traffic between Angola and DRCongo (19841997)», in DE LAME D. \& ZABUS C. (éd.), Changements au féminin en Afrique noire. Anthropologie et littérature, Tervuren, MRCA, Paris, L'Harmattan, pp. 87-114.

- DE BOECK F. (1999a), «Zombification en de postkolonie: een opstel over geschiedschrijving, herinnering en dood in Congo-Kinshasa», Bull. ARSOM, 41, 3, pp. 285-304

- DE BOECK F. (2000a), «'De pandemonium wereld'. Kinderen en hekserij in R.D.
Congo», Medische Antropologie, 12, 1, pp. 4-22.

- DE BOECK F. (2000b), «Le 'deuxième monde' et les enfants-sorciers en République démocratique du Congo", Politique africaine, 80, pp. 32-57.

- DE BOECK F. (2001a), “Comment dompter diamants et dollars: dépense, partage et identité au sud-ouest du Zaïre», in MONNIER L., JEWSIEWICKI B. \& DE VILLERS G. ( 2001), Chasse au diamant au Congo/Zaïre, Cahiers Africains, Afrika Studies, 45-46, pp. 171-208.

- DE BOECK F. (2001b), "Garimpeiro Worlds: Digging, Dying and 'Hunting' for Diamonds in Angola», Review of African Political Economy, 90, pp. 549-562.

- DE BOECK F. (2001c), «'Des chiens qui brisent leur laisse': mondialisation et inversion des catégories de genre dans le contexte du trafic de diamant entre l'Angola et la République démocratique du Congo", in MONNIER L., JEWSIEWICKI B. \& DE VILLERS G. ( 2001), Chasse au diamant au Congo/Zaïre, Cahiers Africains, Afrika studies, 45-46, pp. 209-232.

- DE BOECK F. (2001d), «Dancing the Apocalypse in Congo: Time, Death and Double in the realm of the apocalyptic Interlude", Millenarian Movements in Africa and the Diaspora, Bull. ARSOM, 47 (suppl.), in cooperation with Belgian Association of Africanists, pp. 55-76.

- DE BOECK F. (2005), «The Divine Seed. Children, Gift and Witchcraft in the Democratic Republic of Congo", in $\mathrm{HON}$ WANA A. \& DE BOECK F. (eds.), Makers \& Breakers. Children \& Youth in Postcolonial Africa, Oxford, James Currey, Trenton, Africa World Press, Dakar, Codresria, pp. 188-214.

- DE BOECK F. (2006), "Youth, Death and the Urban Imagination. A case from Kinshasa», Bull. ARSOM, 52, 2, pp. 113-125. - DE BOECK F. \& DEVISCH R. (1995), «Alimentations 'bonnes à penser' et 'bonnes à développer'», in DEVISCH R., DE BOECK F., JONCKERS D. (dir.), Alimentation, traditions et développement en Afrique intertropicale, Paris, L'Harmattan, pp. 9-41.

- DE BOECK F. \& HONWANA A. (2000), «Faire et défaire la société: enfants, jeunes et politique en Afrique», Politique 
africaine, 80 , pp. 5-11

- DE BOECK F. \& PLISSART M.-F. (2005), Kinshasa: récits de la ville invisible, Bruxelles, Editions Luc Pire, 288 p. (version anglaise sous le titre: Kinshasa. The imaginery City).

- DE BOECK F., VAN SYNGHEL K. \& LOMBUNE KALIMASI V. (2005), De gesproken stad: gesprekken over Kinshasa, Kessel-Lo, Literarte, 210 p. (avec un DVD).

- DE BOECK F., voir DEVISCH R. (1995).

- dE CLIPPELE C., voir BOURDOUX P. (2005).

- DE DAPPER M. (ed.) (2004), Tropical Forests: A State of the Art at the Turn of the Century, ARSOM, $143 \mathrm{p}$.

- DE DAPPER M. (ed.) (2005), International Symposium Tropical Forests in a Changing Global Context, Brussels, 8-9 November 2004, UNESCO-MAB, ARSOM, $350 \mathrm{p}$

- DE DAPPER M. \& DE LAME D. (guest eds.) (2006), Africa's Great Rift: Diversity and Unity, International Conference, Brussels, 29-30 September 2005, Africa Tervuren, ARSOM, $300 \mathrm{p}$.

- DE DAPPER M., voir ALEXANDRE J. (1994), DEMARÉE G. (1998).

- DEDEURWAERDER M., voir ARBYN M., (1995).

- DE DONCKER F., voir VAN DEN HERREWEGEN M. (2006).

- DE DORLODOT Ph. (1995), Les réfugiés rwandais à Bukavu au Zaïre. De nouveaux Palestiniens?, Groupe Jérémie, Paris, L'Harmattan, $254 \mathrm{p}$.

- DEELSTRA H. (2001), «Bijdrage tot de ontstaansgeschiedenis van de universiteiten in Congo", Bull. ARSOM, 47, 2, pp. 177-186.

- DEGAND J., voir VERHAEGEN E. (1993).

- DE GRAEVE G., voir TELLIER V. (1996).

- DE HERDT T. (2004), «L'évolution de la pauvreté monétaire à Kisenso entre 19972002. Que pouvons-nous dire, ou vaut-il mieux se taire?», Vivre et survivre à Kinshasa. Problématique du développement humain, pp. 211-252.

- DE HERDT T. et MARYSSE S. (1996), L'économie informelle au Zaïre. (Sur)vie et pauvreté dans la période de transition, Cahiers Africains, Afrika Studies, 21-22, Bruxelles, Institut Africain-CEDAF, Paris,
L'Harmattan, $194 \mathrm{p.}$

- DE HERDT T. \& MARYSSE S. (2002), «La réinvention du marché par le bas. Circuits monétaires et personnes de confiance dans les rues de Kinshasa», in DE VILLERS G., JEWSIEWICKI B. \& MONNIER L. (éd.) (2002), Manières de vivre: économie de la "débrouille» dans les villes du Congo/Zaïre, Cahiers africains, Afrika Studies, 49-50, pp. 171-194.

- DE HERDT T., voir MARYSSE S. (1994).

- DE HEUSCH L. (1995), "La beauté est ailleurs: pour en finir avec les masques tetela. Notices d'histoire et d'ethnographie nkutshu», in DE HEUSCH L. (textes réunis par), Objets-signes d'Afrique, Tervuren, MRCA, Annales, Sciences humaines, 145, pp. 175- 206.

- DELACOLLECTE C., TAELMAN H. \& WÉRY M. (1995), «An etiologic study of hemoglobinuria and blackwater fever in the Kivu Mountain, Zaire», Annales de la Société belge de Médecine tropicale, 75, pp. 33-41.

- DELACOLLECTE C., voir VAN BORTEL W. (1996).

- DE LAME D. (1993), «Instants retrouvés. Rwanda, regards neufs au fil du temps», Liber Amicorum Marcel d'Hertefelt, Essais anthropologiques (sous la dir. de WYMEERSCH P.), Cahiers Africains, Afrika Studies, 4-5, Bruxelles, Institut Africain-CEDAF, pp. 115-131.

- DE LAME D. (1995), «La bière en bouteille et le lait de la houe, paraboles d'une colline rwandaise", in DEVISCH R., DE BOECK F., JONCKERS D. (dir.) (1995), Alimentation, traditions et développement en Afrique intertropicale, Paris, L'Harmattan, pp. 116-153.

- DE LAME D. (1996), Une colline entre mille ou le calme avant la tempête. Transformations et blocages du Rwanda rural, Tervuren, MRCA, Annales, Sciences humaines, 154, $358 \mathrm{p}$.

- DE LAME D. (1997), "L'étude anthropologique comme rencontre interculturelle, une expérience rwandaise», Bull. ARSOM, 43, 4, pp. 495-510.

- DE LAME D. (1999), «Changing Rwandan vision of woman and land, in the heart of the house, at the outskirts of the world», Afrika Focus, 15, 1-2, pp. 3-12.

- DE LAME D. (2003), «Deuil, commé- 
moration, justice dans les contextes rwandais et belge. Otages existentiels et enjeux politiques», Politique africaine, 92, pp. 39-55.

- DE LAME \& DIBWE DIA MWEMBU D. (éd.) (2005), Tout passe. Instantanés populaires et traces du passé à Lubumbashi, Tervuren, Africa Museum, Paris, L'Harmattan.

- DE LAME D., voir DE DAPPER M. (2006). - DE LANGHE E., SWENNEN R. \& VUYLSTEKE D. (1994-1995), "Plantain in the Early Bantu World», Azania, XXIX-XXX, pp. 147-160.

- DELBART V. \& WOLFF E. (2002), «Extension urbaine et densité de la population à Kinshasa: contribution de la télédétection satellitaire», Belgeo, 1, pp. 45-59.

- DELCARTE E., voir AMBE G.A. (1999)

- DELHAL J. (2001), «Les sciences de la Terre», in HALLEUX R. et al. (dir. scientifique), Histoire des sciences en Belgique 1815-2000, vol. 2, ch. 14, Les sciences d'outre-mer, Bruxelles, La Renaissance du Livre, Dexia, pp.250-255. - DEMARÉE G., ALEXANDRE J., DE DAPPER M. (guest eds.) (1998), International Conference on Tropical Climatology, Meteorology and Hydrology, In Memoriam Franz Bultot, Brussels, 22-24 May 1996, ARSOM, 798 p.

- demarée G., DE DAPPER M., ALEXANDRE J. (eds.) (2004), Second International Conference on Tropical Climatology, Meteorology and Hydrology. Climate-related Risks Analysis and Sustainable Development in Tropical Areas, Brussels, 13-14 December 2001, Royal Meteorological Institute of Belgium, ARSOM, $357 \mathrm{p}$.

- DEMARÉE G., DERASSE S. \& ASSANI A. (1998), «Extreme Value Distributions of the Rainy Season Maximum Daily Precipitation Depths at Lubumbashi, Zaire», International conference on Tropical Climatology, Meteorology and Hydrology, In Memoriam Franz Bultot, Brussels, May 1996, ARSOM, pp.507515.

- DEMARÉE G. \& MOHYMONT B. (2004), «Intensity-Duration-Frequency (IDF) Curves for Yangambi, Congo, based upon Longterm High-frequency Precipitation Data Set.
Part I: The History of the Observations and the Data (Demarée G.R.); Part II: The Establishment of Intensity-DurationFrequency Curves for Precipitation in Yangambi, Congo (Mohymont B. et Demarée G.R.)», in DEMARÉE G. et al., Second International Conference on Tropical Climatology, ARSOM, pp. 245-265. - DE MARET P. (1995), "Histoires de croisettes", in DE HEUSCH L. (textes réunis par), Objets-signes d'Afrique, Tervuren, MRCA, Annales, Sciences humaines, 145, pp. 133-145.

- de MARET P. \& LEGROS H. (1993), «Tippo Tip à Mulongo. Nouvelles données sur le début de la pénétration araboswahili au Shaba», in THOVERON G. \& LEGROS H., éditeurs, Mélanges Pierre Salmon, II, pp. 377-401; Civilisations, XLI, 1-2, pp. 377-401

- de maReT P., BAHUChet S., GRENARD Fr. \& GRENARD P. (2002), «Forêts des tropiques, forêts anthropiques», Bull. ARSOM, 48, 2, pp. 97-114; voir aussi une communication des mêmes auteurs et avec le même titre in DE DAPPER M. (ed.), Tropical Forests: a State of the Art at the Turn of the Century, ARSOM, pp. 9-30.

- de maret P., DE MEROdE E., NZABANDORA NDI MUBANZI J., MANGALA BATUKEZANGA R., TREFON T., WEIS G. (2000), «République démocratique du Congo", in BAHUCHET S. \& DE MARET P. (éd. scientifiques), Les Peuples des Forêts Tropicales Aujourd'hui, Bruxelles, Programme Avenir des Peuples des Forêts Tropicales (APFT), Volume III, Région Afrique Centrale, pp. 397-455.

- DE MEESTER T. (1998), «Nationaliteit in Belgisch Congo: constructie en verbeelding", Afrika Focus, 14, 1, pp. 7-16.

- DEPAEPE M. (1994), Kongo, een tweede Vaderland. De kolonie in het onderwijs en het onderwijs in de kolonie (1908-1960), leper, Stedelijk Onderwijsmuseum

- DERASSE S., voir DEMAREE G. (1998).

- DEROLEZ C. (2006), «Les Lole. Une tribu Tetela (R.D.Congo)», Annales Aequatoria, 27, pp. 219-364.

- DE SAINT MOULIN L. (1993), «Cent ans de présence jésuite en Afrique centrale», 
Zaïre-Afrique, pp. 545-571.

- DE SAINT MOULIN L. (1995a), «L'évolution des densités de la population du Zaïre», RBG, 119, 1-2, pp. 95-102.

- DE SAINT MOULIN L. (1995b), «La perception de la démocratie et des élections dans la zone de Matete à Kinshasa", Zaïre-Afrique, 274, pp. 453-474.

- DE SAINT MOULIN L. (1996a), «Kinshasa, trente ans après. Une enquête sur la perception sociale de la justice», Zaïre-Afrique, 305, pp. 197-220.

- DE SAINT MOULIN L. (1996b), «Les problèmes sociaux à Kindele (Kinshasa)», Zaïre-Afrique, 309, pp. 489-508. - DE SAINT MOULIN L. (1998), «La mondialisation dans la Commune de Ngaba à Kinshasa», Congo-Afrique, 327, pp. 418442.

- DE SAINT MOULIN L. (1998), «Conscience nationale et identités ethniques. Contribution à une culture de la paix», Congo-Afrique, 330, 12 figures, pp. 587-630. L'article a été réédité, avec le même titre, dans Congo-Afrique (2003), 372, pp. 93-136.

- DE SAINT MOULIN L. (1999), «Les obstacles majeurs à l'avènement de la démocratie en RDC», in SABAKINU KIVILU (dir.), Démocratie et paix en République démocratique du Congo, Presses de I'Université de Kinshasa, pp. 57-69.

- dE SAINT MOULIN L. (2002), «L'évolution du sentiment ethnique et son rôle dans les conflits actuels en République Démocratique du Congo", Bull. ARSOM, 48, 4, pp. 457-471.

- DE SAINT MOULIN L. (2005), «Essai de recensement et perception des élections dans la commune de Ngaba à Kinshasa», Congo-Afrique, 399, pp. 480-506.

- de SAINT MOULIN, L. (2006a), «Analyse par territoire et ville des résultats de l'enrôlement des électeurs et du référendum sur le projet de Constitution», CongoAfrique, 402-403, pp. 9-34.

- DE SAINT MOULIN, L. (2006b), «Les leçons du premier tour de l'élection présidentielle en RDC», Congo-Afrique, 408, pp. 332-354.

- DE SAINT MOULIN, L. (2007a). «Analyse des résultats du second tour de l'élection présidentielle en RDC», Congo-Afrique,
411, pp. 37-54.

- DE SAINT MOULIN, L. (2007b), «Croissance de Kinshasa et transformation du réseau urbain en République démocratique du Congo depuis l'indépendance», in VELLUT, J.-L. (dir.), Villes d'Afrique. Explorations en histoire urbaine, Cahiers Africains, 73, pp.40-65. - DE SAINT MOULIN L., KABONGO MPUNGA (2000), «La perception des identités et des responsabilités sociales à Kinshasa. Résultats d'une enquête au sud des communes de Lemba et de Kisenzo", Congo-Afrique, 347, pp. 355379.

- DE SAINT MOULIN (L.), avec la coll.de KALOMBO TSHIBANDA (J.-L.) (2005), Atlas de l'organisation administrative de la République Démocratique du Congo, Kinshasa, Centre d'Etudes pour l'Action Sociale CEPAS, $239 \mathrm{p}$.

- DE SAINT MOULIN L., WOLFF E. (2006), «Les résultats de l'élection présidentielle en RDC», Belgeo, 4, pp. 465-471.

- DES FORGES A.L. (1999), Aucun témoin ne doit survivre: le génocide du Rwanda, rédigé par Alison Des Forges sur la base des recherches d'Alison Des Forges et al., Human Rights Watch, Fédération Internationale des Ligues des Droits de l'Homme, Paris, Karthala, 931 p. (le titre de la version anglaise est: Leave none to tell the story).

- DESLAURIER Ch. (2003), «Le "bushingantahe" peut-il réconcilier le Burundi?», Politique africaine, 92, pp. 76-96.

- DESLAURIER Ch. (2003), «Le bushingantahe au Burundi. De l'institution locale au patrimoine institutionnel national», in PERROT CI.-H. \& FAUVELLE-AYMAR F.-X. (dir.), Le retour des rois. Les autorités traditionnelles et l'Etat en Afrique contemporaine, Paris, Karthala, pp. 401-417.

- DE VILLERS G. (dir.) (1994), BelgiqueZaïre. Une histoire en quête d'avenir, Actes des rencontres de Bruxelles (ULB, 7-8-9 octobre 1993) organisées par l'Institut Africain, le Centre National de Coopération au Développement, l'Association Belge des Africanistes, Cahiers Africains, 9-1011, Bruxelles, Institut Africain-CEDAF, Paris, L'Harmattan, $349 p$

- DE VILLERS G. (1995a), De Mobutu à Mobutu: trente ans de relations Belgique- 
Zaïre, Bruxelles, De Boeck-Wesmael, 255 p. - DE VILLERS G. (1995b), “L'”Africanisme" belge face aux problèmes d'interprétation de la tragédie rwandaise", Politique Africaine, 53, pp. 121-132.

- DE VILLERS G. (1995c), «Les études africaines en Belgique. Evolution et perspectives», Bull. ARSOM, 41, 3, pp. 329349.

- de VILLERS G. (dir.) (1996), Phénomènes informels et dynamiques culturelles en Afrique, Actes des Journées d'étude organisées les 16 et 17 décembre 1994 à Bruxelles par l'Association Belge des Africanistes, avec la collaboration de I'Institut Africain-CEDAF, Cahiers Africains, 19-20, Bruxelles, Institut Africain-CEDAF, Paris, L'Harmattan, 286 p.

- DE VILLERS G. (2000), «Formes et dynamiques de l'informel», in MONNIER L. et DROZ I. (dir.), Côté jardin, côté cour: l'anthropologie de la maison africaine, Paris, P.U.F., pp. 247-263.

- DE VILLERS G. (2009), République démocratique du Congo. De la guerre aux élections. L'ascension de Joseph Kabila et la naissance de la Troisième République (janvier 2001-août 2008), Cahiers africains, 75, Africa Tervuren, Paris, L'Harmattan, $478 \mathrm{p}$

- DE VILLERS G., JEWSIEWICKI B. et MONNIER L.(éditeurs) (2002), Manières de vivre: économie de la "débrouille" dans les villes du Congo/Zaïre, Cahiers africains, 49-50, Tervuren, Institut AfricainCEDAF, Paris, L'Harmattan, 205 p.

- DE VILLERS G. \& OMASOMBO TSHONDA J. (1997), Zaïre. La transition manquée: 1990-1997, Cahiers Africains, $27-$ 28-29, Tervuren, Institut Africain-CEDAF, Paris, L'Harmattan, $302 \mathrm{p}$.

- DE VILLERS G. \& WILLAME J.-C. (en coll. avec OMASOMBO J. et KENNES E.) (1998), République démocratique du Congo. Chronique politique d'un entredeux-guerres. Octobre 1996-Juillet 1998, Cahiers Africains, 35-36, Tervuren, Institut Africain-CEDAF, Paris, L'Harmattan, 371 p. - DE VILLERS G. (en coll. avec OMASOMBO J. ET KENNES E.) (2001), République démocratique du Congo. Guerre et politique. Les trente derniers mois de L.D. Kabila (août 1998-janvier 2001), Cahiers Africains, 47-48, Tervuren, Institut
Africain, Paris, L'Harmattan, 346 p.

- DEVISCH R. (1994), «De spiegeling voorbij: de verdorpelijking van Kinshasa, Bull. ARSOM, 40, 2 (publié en 1995), pp. 141-165

- DEVISCH R. (1995a), «La villagisation de Kinshasa, RBG, 119, 1-2, pp. 115-121. - DEVISCH R. (1996), "Le pillage de Jésus": les églises de guérison et la villagisation de Kinshasa, in DE VILLERS G. (dir.), Phénomènes informels et dynamiques culturelles en Afrique, Cahiers d'Etudes Africaines, 19-20, Bruxelles, Institut Africain, Paris, L'Harmattan, pp. 91-138.

- DEVISCH R. (1998), «La violence à Kinshasa, ou l'institution en négatif», Cahiers d'études africaines, 150-152, pp. 441-469.

- DEVISCH R. (1999a), «Les Eglises de guérison à Kinshasa et la villagisation matricentrée de la ville, in DE LAME D. et ZABUS Ch., Changements au féminin en Afrique Noire. Anthropologie et littérature, vol. I., Anthropologie, Paris, L'Harmattan, pp. 203-214.

- DEVISCH R. (1999b), «Les universités en Afrique noire et les savoirs endogènes, Bull. ARSOM, 45, 3, pp. 261-293).

- DEVISCH R., DE BOECK F., JONCKERS D. (dir.) (1995), Alimentation, traditions et développement en Afrique intertropicale, Paris, L'Harmattan, $303 \mathrm{p}$.

- DEVISCH R., voir VAN WOLPUTTE S. (2002).

- DEWAELE St., voir MUCHEZ Ph. (2006).

- DE WASSEIGE C. (2006), «Utilisation des images satellites pour le suivi de la déforestation dans le Parc national des Virunga», in DE DAPPER M. \& DE LAME D. (eds.), Africa's Great Rift. Unity and Diversity, Africa-Tervuren, ARSOM, p. 522.

- DIBANGA B. \& ALEXANDRE J. (1996), «Une terrasse fluviale associée à un seuil rocheux dans une région tropicale à saison sèche (la Kafubu à Kamibikila, Shaba, Zaïre)», Bull. SOBEG, LXV, 1, pp. 35-47.

- DIBWE DIA MWEMBU (1993), «La mortalité infantile à l'Union Minière du HautKatanga. Une analyse préliminaire (19291970)», Zaïre-Afrique, 278, pp. 487-500.

- DIBWE DIA MWEMBU (1993), «La fonc- 
tion des femmes africaines dans les camps de travailleurs de I'Union Minière du Haut-Kartanga (1925-1960)», ZaïreAfrique, 272, pp. 105-118.

- DIBWE DIA MWEMBU D. (2001), Bana Shaba abandonnés par leur père: structures de l'autorité et histoire sociale de la famille ouvrière au Katanga 1910-1997, Paris, L'Harmattan, $259 \mathrm{p}$

- DIBWE DIA MWEMBU D. (2004), «Le poids des sources orales dans l'écriture et la réécriture de l'histoire contemporaine au Katanga", in MABIALA MANTUBANGOMA P. (dir.), La Nouvelle Histoire du Congo. Mélanges eurafricains offerts à Frans Bontinck, C.I.C.M., Cahiers Africains, 65-66-67, pp. 35-46.

- DIBWE DIA MWEMBU D. (2005), «Relecture de l'histoire et transformation des rapports entre les Katangais et les Kasaïens du Katanga», in DIBWE DIA MWEMBU D., NGANDU MUTOMBO M., Vivre ensemble au Katanga, préface d'E. Kennes, Paris, L'Harmattan, pp. 15-178.

- DIBWE DIA MWEMBU D. (2007), «Lubumbashi: histoire et mémoire d'une ville industrielle», in VELLUT J.-L. (dir.), Villes d'Afrique. Explorations en histoire urbaine, Cahiers Africains, 73, Africa Tervuren, F.N.R.S., Chaire d'études africaines de I'UCL, Paris, L'Harmattan, pp.131-144.

- DICKENS E., voir LELO NZUZI (2004).

- DIKUMBWA N. \& MBENZA M. (1999), «A propos de l'origine des îlots de forêt dense sèche zambézienne du Katanga méridional», GEO-ECO-TROP, 23, pp. 1530.

- DONNEN Ph., voir HENNART Ph. (1996), PORIGNON D. (2006).

- DOUGLAS M. (1999), «Sorcery accusations unleashed: the Lele revisited", Africa, 69, 2, pp. 177-193.

- DOUMENGE C. (1998), «Forest diversity, distribution and dynamics in the Itombwe Mountains, South-Kivu, Congo Democratic Republic», Mountain Research and Development, USA, 18, 3, pp. 249-264.

- DOUTRELEPONT H. (2000), voir MER-

CADER J.

- DRACHOUSSOFF V. (1994), «Afrique centrale: un pari sur l'avenir?», Bull. ARSOM, 40, Suppl. $\mathrm{n}^{\circ}$ 1, pp. 67-81.
- DRAMAIX M., voir MUGISHO E. (2002), PORIGNON D. (2006).

- DRIESMANS I. (2002), "L'évolution de l'économie marchande des paysans africains de Lisala (Congo belge) et du Haut-Ogooué (Moyen-Congo) pendant la crise des années trente», Revue Belge d'Histoire Contemporaine, XXXII, 3-4, pp. 459-496.

- DUCHAUFOUR H., voir SIMONARD (1994).

- DUMONT P., HANON M. (1997), «Le Plateau des Kundelungu, paléograben ou aulacogène?", in CHARLET J.-M. (guest ed.) (1997), Colloque international Cornet, 5-9 septembre 1994, Mons, Faculté polytechnique de Mons et ARSOM, pp. 51-89. - D'ZOUZA S., MAKAYA MBOKO MBENZA \& KALENDI MUHNIDO (1995), «Evaluation du niveau de pauvreté: cas de la Zone de Matete», Zaïre-Afrique, 294, pp. 219-236.

- EALE B. J.-Cl. (coord.) (2004), 80 ans LEO-KIN 1923-2003. Cheminement d'une capitale, Ed. CMCT FCB, $100 \mathrm{p}$.

- EHESS (éd.) (1998), «Disciplines et déchirures. Les formes de la violence», Cahiers d'études africaines, 150-152, $526 \mathrm{p}$

- ENGELS P., voir TSHIBANGU K. (1997).

- ERPICUM M., voir KALOMBO (1996)

- ERPICUM R., Dr LUZOLELE LOLA NKALALA (1998), «Table ronde sur la lutte contre la pauvreté, Kinshasa, 22-24 janvier 1998», Congo-Afrique, 235, pp. 270-280.

- ERRERA M., voir TACK L. (2006)

- EWANGO C.E.N. (2000), voir MERCADER J.

- FALANGANI MYONDO PASHI (2002), «Paupérisation des familles petites-bourgeoises et transformations des valeurs en période de crise», in DE VILLERS G., JEWSIEWICKI B. \& MONNIER L. (éd.) (2002), Manières de vivre: économie de la "débrouille» dans les villes du Congo/ Zaïre, Cahiers africains, 49-50, pp. 113140.

- FERNANDEZ-ALONSO M., voir TACK L. (2006).

- FRANCESCHI G., voir TACK L. (2006).

- FRANÇOIS A. (1997), «Etude géologique de l'Arc cuprifère du Shaba. Progrès réalisés entre 1950 et 1980», in 
Colloque International Cornet, Fac. Polyt. Mons et ARSOM, pp. 21-50.

- FUKUYAMA K., voir SANGA-NGOIE K. (1996),

- GAHAMA J. (dir.) (1998), Démocratie, bonne gouvernance et développement pour une paix durable dans la Région des Grands Lacs: contribution des universités et des universitaires, Actes du colloque de mai 1998 à Bujumbura, Bujumbura, Ed. Université du Burundi, $402 \mathrm{p}$.

- GAHAMA J. (2001), Le Burundi sous administration belge: la période du mandat 1919-1939, 2e édition, Paris, Karthala, $467 \mathrm{p}$.

- GAHAMA J. \& THIBON C. (dir.) (1994), Les régions orientales du Burundi. Une périphérie à l'épreuve du développement, Paris, Karthala, 638 p.

- GAHAMA J., voir CHRÉTIEN J.-C. (1995).

- GAKINAHE G. (1993), «Les transports sur le lac Kivu», L'eau et l'aménagement dans l'Afrique des Grands lacs, Colloque Bujumbura 1991, Coll. Pays enclavés, n5, CRET, Talence, pp. 309-324.

- GALLEZ A. \& VAN RANST E. (1997), «Intensification de la production vivrière et aptitude des terres au Rwanda. Etat de la question et perspectives de développement», in VERHASSELT $Y$. (Actes édités par), Rwanda-Burundi, Journée d'étude, Bruxelles, ARSOM, pp. 35-61.

- GALLEZ D., éditeur (2001), Fernand Allard. Journal du Congo (1905-1907), un apprentissage missionnaire, Institut Belge de Rome, Bruxelles, 338 p.

- GARIN B., JEANNEL D., KAZADI K., COMBE P., SINGA L. \& DE THÉ G. (1993), «Introduction of HIV1 in a rural city of Zaire», Annales de la Société belge de Médecine tropicale, 73, pp. 143-147.

- GASHAGAZA J.-B.M., voir KANANGIRE C.K (2001), MICHA J.-C. (2002).

- GATERA F. (1993), "L'utilisation de l'eau à la maison dans la commune de Nyamutera (Rwanda)», L'eau et l'aménagement dans l'Afrique des Grands Lacs, Colloque Bujumbura 1991, pp. 177191.

- GAUD M. (dir.) (1997a), Du Zaïre au Congo, Afrique contemporaine, 183, Dossier spécial, Paris, La Documentation
Française.

- GAUD M. (1997b), «Chronologie politique du Congo-Zaïre-Congo», Du Zaïre au Congo, Afrique contemporaine, 183, pp. 67-87.

- GAUSSET Q. (2001), «Aids, Herald of the Apocalypse», Bull. ARSOM, 47, Suppl., pp. 143-144.

- GELADÉ V. (2002), «Les débuts de la navigation à vapeur sur le Haut-Congo (1882-1898)», Revue Belge d'Histoire Contemporaine, XXXII, 3-4, pp. 383-418.

- GELLENS D., voir BULTOT F. (1994).

- GIRALOWSKI J. (2002), La déforestation en Afrique centrale. Les facteurs de la dégradation des forêts denses humides équatoriales dans la République démocratique du Congo, Varsovie, Editions Académiques DIALOG, 82 p., 3 cartes h.-t.

- "Gisements stratiformes de cuivre et minéralisations associées», voir Colloque international Cornet et CHARLET J. (1997).

- GOMA EPIDEMIOLOGY GROUP (1995), «Public health impact of Rwandan refugee crisis; what happened in Goma, Zaire, in July 1994», The Lancet, 345, pp. 339-344. - GOMMES R. (1998), «Some Aspects of Climate Variability and Food security in sub-saharan Africa», International Conference on Tropical Climatology, Meteorology and Hydrology, In Memoriam Franz Bultot, Brussels, May 1996, ARSOM, pp. 665-673.

- GONDOLA C.D. (1993), «Musique moderne et identités citadines, le cas du Congo-Zaïre, Afrique contemporaine, 168, numéro spécial "Villes d'Afrique» (sous la dir. de P. Hugon et de R. Pourtier), La documentation française, pp. 155-168.

- GONDOLA C.D. (1997), Villes miroirs. Migrations et identités urbaines à Brazzaville et Kinshasa 1930-1970, Paris, L'Harmattan, $455 \mathrm{p}$.

- GONDOLA C.D. (2002), The History of Congo, Westport, Conn., Grenwood Press, XVIII + $215 \mathrm{p}$.

- GOOSSENS F. (1996), Cassava production and marketing in Zaire. The market of Kinshasa, Leuven, Leuven University Press, $178 \mathrm{p}$.

- GOOSSENS F., MINTEN B., TOLLENS E. 
(1994), Nourrir Kinshasa. L'approvisionnement local d'une métropole africaine, Coll. Zaïre. Histoire et Société, préface de G. Verhaegen, KULeuven, Paris, L'Harmattan, $403 \mathrm{p}$.

- GOOSSENS P. (2007), «Phoenix Rising in an Uncertain World. New Mining Activities in Katanga», Bull. ARSOM, 53, 3, pp. 361-385.

- GOTANĖGRE J.F. (1993), «Analyse géographique de l'incidence du V.I.H. et du SIDA au Rwanda en 1990», Les Cahiers d'Outre-Mer, 46, n¹83, pp. 233-252.

- GOTANĖGRE J.F. (1996), «Surpopulation et guerre: l'exemple du Rwanda", Afrique et îles de l'Océan indien. La difficile maîtrise des densités, Bull. de la Société languedocienne de géographie, 30, 1-2, pp. 33-55.

- GOUROU P. voir NICOLAÏ H. (1996).

- GOYENS P. (1997), «Rwanda en Burundi: sanitaire toestand", in VERHASSELT Y. (Actes édités par), RwandaBurundi. Journée d'étude, Bruxelles, ARSOM, pp. 81-96.

- GOYENS P., PORIGNON D., MUGISHO SORO'GANE E., TONGLET R., HENNART Ph., VIS H.L. (1996), «Humanitarian Aid and Health Services in Eastern Kivu, Zaire. Collaboration or Competition?», Journal of Refugee Studies, 9, 3, pp. 268280.

- GOYENS P. voir BRASSEUR D. (1998), VIS H. (1994, 1996, 1997).

- GROOTAERS J.-L. (1995), «"Shifting civilizers" among "shifting cultivators": a history of agricultural development in Central African Zandeland», in DEVISCH R., DE BOECK F., JONCKERS D. (dir.) (1995), Alimentation, traditions et développement en Afrique intertropicale, Paris, L'Harmattan, pp.155-179.

- GROOTAERS J.-L. (dir.) (1998), Mort et maladie au Zaïre, Cahiers Africains, 3132, Collection «Zaïre, Années 90», vol. 8, Bruxelles, Institut Africain-CEDAF, Paris, L'Harmattan, $172 \mathrm{p}$.

- GROOTAERS J.L. (1999), «Zande Prophetesses at the Articulation of Local Culture and World Religion», in DE LAME D. \& ZABUS Ch., Changements au féminin en Afrique Noire. Anthropologie et littérature, Paris, L'Harmattan, vol. I, pp. 183-201.
- GUICHAOUA A. (1995), Les crises politiques au Burundi et au Rwanda, 19931994, analyses, faits et documents, Villeneuve d'Ascq, Université des sciences et technologies de Lille, $794 \mathrm{p}$.

- GUICHAOUA A. (dir.) (2004), Exilés, réfugiés, déplacés en Afrique centrale et orientale, Paris, Karthala, 1066 p.

- GUICHAOUA A. (2005), Rwanda 1994: les politiques du génocide à Butare, Paris, Karthala, $497 \mathrm{p}$.

- GUISSET J. (dir.) (2003), avec la coll. du MRAC, Le Congo et l'Art belge 18801960, Tournai, La Renaissance du Livre, $280 \mathrm{p}$

- HABIYAREMYE F.X., ROCHE E. (2003), «Incidence anthropique sur le milieu montagnard du graben centrafricain: complément phytodynamique aux interprétations palynologiques», GEO-ECO-TROP, 27, 12, pp. 53-62.

- HAHN B. H., voir SHARP P.M. (2008).

- HALEN P. (1993), Le petit Belge avait vu grand: une littérature coloniale, Bruxelles, Ed. Labor, 397 p.

- HALEN P. \& RIESZ J. (1993b), Images de l'Afrique et du Congo dans les lettres françaises de Belgique et alentour, Actes du colloque de Louvain-la-Neuve, 4-6 février 1993, Bruxelles, Tetxyles, 372 p.

- HALEN P. \& RIESZ J. (textes réunis par) (1995), Littératures du Congo-Zaïre, Actes du colloque international de Bayreuth, 22-24 juillet 1993, Amsterdam, Rodopi, XOV-424 p.

- HALLEUX R., VANDERSMISSEN J., DESPY-MEYER A., VANPAEME G. (direction scientifique) (2001), Histoire des sciences en Belgique 1815-2000, vol. 2, Bruxelles, La Renaissance du Livre, Dexia, 312 p. (voir ch. 14, Les sciences d'outre-mer, pp. 235-265).

- HANON M., voir DUMONT P. (1997)

- HARROY J.-P. (1993), "Contribution à l'histoire jusque 1934 de la création de I'Institut des parcs nationaux du Congo Belge, in Mélanges Pierre Salmon II, Histoire et ethnologie africaines, Civilisations, XLI, 1-2, pp. 427-442.

- HECQ J. (1994), «Recherche et conservation», Bull. ARSOM, 40, Suppl. $\mathrm{n}^{\circ}$ 1, pp. 83-94.

- HEINTZE B. (2004), «Le voyage d'exploration de Max Buchner au royaume 
lunda 1878-1882», in MABIALA MANTUBA-NGOMA P. (dir.), La Nouvelle Histoire du Congo, Cahiers Africains, 65-66-67, pp. 333-364.

- HENNART Ph., PORIGNON D. \& DONNEN Ph. (1996), «La nutrition et le couple mère-enfant», Symposium "L'Enfance dans le Tiers Monde", Bruxelles, 20-22 octobre 1994, Actes publiés sous la direction de SYMOENS J.-J., Bruxelles, ARSOM, pp. 103-114.

- HENNART Ph., voir GOYENS Ph. (1996), MUGISHO E. (2002), PORIGNON D. (1995 et 2006).

- HENRY E. (2008), «Le Mouvement Géographique, entre géographie et propagande coloniale, Belgeo, pp. 27-46.

- HERBOSCH A., voir KASEBA M.K. (1997).

- HIRAUX F. (2007), «Représentation des villes et valeurs urbaines dans la colonie belge: parcours historien dans une collection photographique 1946-1952», in VELLUT J.-L. (dir.), Villes d'Afrique. Explorations en histoire urbaine, Cahiers Africains, 73, Africa-Tervuren, FNRS, Chaire d'Etudes africaines UCL, Paris, L'Harmattan, pp. 113-144.

- HOCHSCHILD A. (1998), King Leopold's Ghost. A Story of Greed, Terror and Heroism in Colonial Africa, Boston, New York, Houghton Mifflin Co, 366 p.

- HOCHSCHILD A. (1998), Les fantômes du Roi Léopold II. Un holocauste oublié, Paris, Belford, 435 p. Une édition de poche en 2007, Les Fantômes du roi Léopold. La terreur coloniale dans l'État du Congo 1884-1908, Texto, Paris, Tallandier, $618 \mathrm{p}$.

- HONWANA A. (2000), «Innocents et coupables: les enfants-soldats comme acteurs tactiques», Politique africaine, 80, pp. 58-78.

- hONWANA A., voir DE BOECK F. (2000).

- hUART A., tOMBU C. (2009), Congo, pays magnifique, Weyrich Africa, $216 \mathrm{p}$.

- HUETZ DE LEMPS C. (1994), «Un potentat arabe, trafiquant d'ivoire et négrier, en Afrique centrale», Les Cahiers d'OutreMer, 47, n¹87, pp. 350-363.

- HUETZ DE LEMPS A. (2001), Boissons et civilisations en Afrique, Collection Grappes et Millésimes, Bordeaux, Presses Univer- sitaires de Bordeaux, $658 \mathrm{p}$.

- HUNT N.R. (2005), «Hommes et femmes, sujets du Congo colonial», in VELLUT J.-L. (dir.), La mémoire du Congo, le temps colonial, Africa-Tervuren, MRCA, Editions Snoeck, pp. 51-57.

- ILIFFE J. (2006), The African AIDS Epidemic. A History, Athens, Ohio University Press, Oxford, James Currey, Cape Town, Double Storey/a Juta Company, $214 \mathrm{p}$.

- ILUNGA L. (1993), «Approvisionnement en eau et en hydro-électricité dans le Bwisha», Colloque de Bujumbura, 1991, Coll. Pays enclavés, $n^{\circ} 5$, Talence, CRET, pp. 342-360.

- ILUNGA L. (2006), «Etude des sites majeurs d'érosion à Uvira (R.D Congo)», GEO-ECO-TROP, 30, 2, pp. 1-12.

- ILUNGA L., LONDA L. (1993), «L'eau et l'aménagement dans la plaine de la Ruzizi, partie zaïroise», L'eau et l'aménagement dans l'Afrique des Grands Lacs, Colloque Bujumbura, 1991, Coll. Pays enclavés, $n^{\circ} 5$, Talence, CRET, pp. 71-90.

- ILUNGA L. \& MUGIRANEZA A. (2006), «Méthode pour déterminer la date probable des semailles à Kigali et ses environs», GEO-ECO-TROP, 30, 2, pp. 13-30. - ILUNGA L., MUHIRE I. \& MBARAGIJIMANA C. (2004), «Saisons pluviométriques et origine des pluies au Rwanda, GEO-ECO-TROP, 28, 1-2, pp. 61-68.

- ILUNGA L., MWININYINKONDO K.B. (1993), «Le régime pluviométrique et le calendrier agricole sur les hautes terres du Kivu (est du Zaïre)», L'eau et l'aménagement dans l'Afrique des Grands Lacs, Colloque 1991, Bujumbura, Coll. Pays enclavés, $\mathrm{n}^{\circ} 5$, Talence, CRET, pp. 43-55.

- ILUNGA L. \& TSHENDA A. (2004), «Facteurs physiques du ruissellement à Kigali (Rwanda)», GEO-ECO-TROP, 28, 1-2, pp. 53-60.

- IMBS F. (1997), «Retours et reconstruction au Rwanda. Et pourtant ils vivent!», Géopolitique d'une Afrique médiane, Hérodote, 86-87, pp. 150-181.

- IMBS F. (2000), «La villagisation au Rwanda: politique d'urgence et politique de développement», in NICOLAï $\mathrm{H}$., PÉLISSIER P. \& RAISON J.-P., Un géo- 
graphe dans son siècle. Actualité de Pierre Gourou, Paris, Karthala, Géotropiques, pp. 259-274.

- IMBS F., BART F. et BART A. (1994), «Le Rwanda: les données socio-géographiques», Hérodote, 72-73, pp. 246269.

- IMERZOUKENE S. \& VAN RANST E. (2001), «Une banque de données pédologiques et son S.I.G. pour une nouvelle politique agricole au Rwanda», Bull. ARSOM, 47, 3, pp. 299-329.

- INDRING'IA.N. \& BAHUMIGA L.B., "L'exploitation artisanale de l'or et du diamant dans le Haut-Zaïre (1982-1995)", Les Cahiers d'Outre-Mer, 202, Bordeaux, pp. 157-170.

- INSONI A. (2007), «Facteurs géographiques et manifestations du VIH/Sida. Cas de la ville de Lubumbashi», Congo-Afrique, 420, pp. 791-815.

- INSTITUT NATIONAL DE LA STATISTIQUE ET BANQUE CENTRALE DU CONGO (2003), «Economie congolaise de 1990 à 2000", Congo-Afrique, 375, pp. 282-291.

- IPALAKA YOBWA J., voir WOLFF E. (2001).

- JACKSON S. (2001), «"Nos richesses sont pillées!" Economie de guerre et rumeurs de crime au Kivu», Politique africaine, 84, p.117-135.

- JACQUES C. et PIETTE V. (2003), «La femme européenne au Congo belge: un rouage méconnu de l'entreprise coloniale. Discours et pratiques (1908-1940)", Bull. ARSOM, 49, 3, pp. 261-293.

- JANSSENS P.G. (1995), «Eugène Jamot et Emile Lejeune. Pages d'histoire», Annales de la Société belge de Médecine tropicale, 75, pp. 1-12.

- JANSSENS P.G. (1996), «La trypanosomiase en Angola à l'aube du $20^{\mathrm{e}}$ siècle. Réflexions sur les épidémies du bassin du Cwanza et du Congo (notes historiques)», Bull. ARSOM, 42, 3, pp. 537569.

- JEANNEL D., voir GARIN B. (1993).

- JEDWAB J. (1999), «Le platine au Kivu. Mystères, mythes, erreurs et vérités autour du platine de Lubero», Bull. ARSOM, 45, 2, pp.177-191

- JEFRENOVAS V. (2000), «Treacherous waters: the politics of history and the pol- itics of genocide in Rwanda and Burundi», Africa, 70, 2, pp. 298-308.

- JEURISSEN L. (2002), «Les ambitions du colonialisme belge pour la "race mulâtre" (1918-1940)", Revue Belge d'Histoire Contemporaine, XXXII, 3-4, pp. 497-535.

- JEURISSEN L. (2003), Quand le métis s'appelait «mulâtre». Société, droit et pouvoir coloniaux face à la descendance des couples eurafricains dans l'ancien Congo belge, Cahiers Migrations, 29, Louvain-la-Neuve, Bruylant-Academia et Sybidi, $120 \mathrm{p}$.

- JEWSIEWICKI, B. (2002), Mami Wata. La peinture urbaine au Congo, Coll. Le Temps des Images, NRF, Gallimard, Paris, $236 \mathrm{p}$.

- JEWSIEWICKI B. (dir.) (2003), Musique urbaine au Katanga: de Malaika à Santu Kimbangu, Paris, L'Harmattan, $180 \mathrm{p}$

- JEWSIEWICKI B. (2004), «(Auto) représentation d'une société "moderne" en (dé)construction. De la modernisation coloniale à la globalisation», in MABIALA MANTUBA-NGOMA P. (dir.), La Nouvelle Histoire du Congo. Mélanges eurafricains offerts à Frans Bontinck, C.I.C.M., Cahiers Africains, 65-66-67, pp. 251-266.

- JEWSIEWICKI B. \& KLAUBER V. (édité par) (2003), Université de Lubumbashi 1990-2002. Société en détresse. Pari sur l'avenir, Paris, L'Harmattan, $241 \mathrm{p}$.

- JEWSIEWICKI B. voir DE VILLERS G. (2002).

- JONCKERS D., voir DEVISCH R. (1995).

- JUAN-TRESSERAS J. (2000), voir MERCADER J.

- KABAMBA B. \& LANOTTE O. (1999), "Guerres au Congo-Zaïre (1996-1999)», in MATHIEU P. \& WILLAME J.-C. (dir.), Conflits et guerres au Kivu et dans la région des Grands Lacs, Cahiers Africains, 39-40, pp. 99-159.

- KABAMBA K. (2000), Relations à la ville et territorialité dans la campagne environnante de Kananga (RD Congo), Thèse de doctorat en géographie, Université de Liège, $179 \mathrm{p}$.

- KABAMBA K. (2002), «Dynamique territoriale du Kasayi (Congo-Kinshasa). Incidences des changements socio-politiques et économiques sur la recomposition spatiale», Bull. Société géographique 
de Liège, 39, 2, pp. 101-114.

- KABAMBA K., LIKENGA C.E. (2002), «La minorité Tetela à Kananga (R.D. du Congo)», Espaces publics et marqueurs culturels dans les villes d'Afrique noire, Géographie et Cultures, 41, pp. 75-92.

- KABAMBA KABATA \& NYOKA MUPANGILA (1998), «Les points de vente périphériques de Kananga. Spécificités, fonctions et attractivité», Bull. Société géographique de Liège, pp. 103-112.

- KABAMBA K., voir MWANZA H. (2002).

- KABAMDA P. (2004), "Où va la valeur ajoutée dans les territoires occupés par les rebelles et comment la Ville de Kisangani en est affectée?», CongoAfrique, 388, pp. 481-504.

- KABANDA T. (1993), «L'eau en région volcanique au nord-ouest du Rwanda: abondance et pénurie", L'eau et l'aménagement dans l'Afrique des Grands Lacs, Colloque Bujumbura 1991, Coll. Pays enclavés, $n^{\circ} 5$, CRET, Talence, p. 5769.

- KABONGO MALU E. (2007), Epurations ethniques en RD Congo (1991-995): la question Luba-Kasaï, Espace Kinshasa, Paris, L'Harmattan, $207 \mathrm{p}$.

- KABONGO MPUNGA, voir DE SAINT MOULIN L (2000).

- KABONYI NZABANDORA C. (2007) «Etude palynologique de la séquence sédimentaire de Musisi-Karashoma II, Sud Kivu. Synthèse de l'évolution environnementale du Sud Kivu au cours des deux derniers millénaires», GEO-ECOTROP, 31, pp. 147-170.

- KABUYA KALALA \& YAV KARL YAV (1994), «L'espace monétaire kasaïen: leçons de la survivance d'une monnaie déchue», Zaïre-Afrique, 290, pp.197-214.

- KABUYA KALALA \& MATATA PONYO MAPON (1999), L'espace monétaire kasaïen. Crise de légitimité et de souveraineté monétaire en période d'hyperinflation au Congo (1993-1997), avec une préface de M. Norro et une postface «De nouveaux espaces monétaires kasaïens sont-ils possibles en RDC? », par Tshiunza Mbive, Cahiers Africains, 41, Tervuren, Institut Africain-CEDAF, Paris, L'Harmattan, $148 \mathrm{p}$.

- KADENDE-KAISER R. M., KAISER P.J. (1997), «Modern Folklore, Identity and
Political Change in Burundi», African Studies Review, 40, 3, pp. 29-54.

- KAFARHIRE MURHULA T. (2003), "L'autre visage du conflit dans la crise des grands Lacs. Mémoire historique sur la crise de la nationalité au Kivu», CongoAfrique, 374, pp. 221-249.

- KAHOLA TABU O., RUBBERS B. (2008), «Entre collaboration et confrontation: l'ambivalence des rapports entre pouvoirs publics et enfants de la rue à Lubumbashi (RDC)», Autrepart, 47, pp. 35-41.

- KAKESE C. (2000), «La géographie religieuse des villes en RDC. Un facteur de sous-développement de l'espace vital», Alternative, 3, Kinshasa, Saint-Paul, pp. 12-15.

- KAKESE K. B., voir KAYEMBE C.M.P. (1993), ASSUMANI S. (1993).

- KAKUDJI A. (2006), «Les enfants en rupture en RDC. Analyse d'une expérience d'enquête collective», in TREFON Th. \& PETIT P. (dir.), Expériences de recherche en République démocratique du Congo, Civilisations, LIV, 1-2, pp. 125-133.

- KALENDI MUHNIDO, voir D'ZOUZA S. (1995).

- KALOMBO K. (2001), «Occurrence des intensités des pluies et leurs effets sur l'environnement dans une région tropicale (région de Lubumbashi, sud-est de la R.D. du Congo)», GEO-ECO-TROP, 25, 14, pp. 17-28.

- KALOMBO K. (1995), «La pluviométrie pendant la période de sécheresse récente (depuis 1980) dans le sud-est du Zaïre», GEO-ECO-TROP, 19, 1-4, pp. 1734

- KALOMBO K., ASSANI A. (1999, publié en 2000), «La pluviométrie de la côte atlantique près de l'embouchure du fleuve Congo: le contact entre le domaine de la mousson atlantique et la zone sous l'influence du courant de Benguela», Publications de l'Association internationale de climatologie, 12, pp. 73-80.

- KALOMBO K., ERPICUM M. \& ASSANI A. A. (1996), «Evolution chronologique des précipitations dans le sud-est du Zaïre. Comparaison avec celle des pays voisins», Publications de l'Association Internationale de Climatologie, 9, pp. 446-453.

- KALOMBO K., voir ASSANI A.A. (1995 
et 1997).

- KALULAMBI PONGO M. (1993), "Christianisme et image de l'Autre en Afrique belge. Les catégories de langage dans les stratégies de domination, Cahiers d'Etudes Africaines, 130, XXXIII2, pp. 275-293.

- KAMAVU DUNGO (2004), «Aperçu sur l'impact des institutions et du changement institutionnel sur la qualité de vie dans une localité: le cas de Kisenso à Kinshasa», Vivre et survivre à Kinshasa. Problématique du développement humain, pp.151-180.

- KANANGIRE C.K., MICHA J.C., GASHAGAZA J.-B. M. (2001), Aménagement des marais au Rwanda, Actes du Colloque Butare, 5-7 juin 2001, Namur, ETEC (Eco-Technologie des Eaux Continentales), Presses Universitaires de Namur, $118 \mathrm{p}$.

- KANENE MPALI SITELA (1992), "L'espace commercial de Kinshasa», Bulletin Géographique de Kinshasa, GEOKIN, III, 2, pp. 179-215.

- KANIMBA MISAGO (1995), «Les Elinga de la Ruki», Annales Aequatoria, 16, pp. 229-258.

- KANYINGINI MWENYIMALI (1993), «La pêche de Limnothrissa miodon dans la région de Bukavu», L'eau et l'aménagement dans l'Afrique des Grands Lacs, Colloque 1991 Bujumbura, Collection Pays enclavés, Talence, CRET, $n^{\circ} 5, \mathrm{pp}$. 247-258.

- KANKONDE MBUYI (1993), «Kinshasa - Problèmes de gestion urbaine, d'infrastructure et d'approvisionnement en nourriture», chapitre 6 de STREN R.F. \& WHITE R.R. (dir.), Villes africaines en crise. Gérer la croissance urbaine au sud du Sahara. Côte d'Ivoire,-Kenya-NigeriaSénégal-Tunisie-Zaïre, Coll. Villes et entreprises, Paris, L'Harmattan, 288 p. (il s'agit de l'édition française d'un livre publié en anglais chez Westview Press).

- KANYINGINI B., MICHA J.-C., VAN DEN HAYTE J., PLATTEAU J.P., WATONGOKA H., MÉLARD C, WILONDIA M.K et ISHIMBISHO M. (1999), Pêche du sambaza au filet maillant dans le lac Kivu, Presses Universitaires de Namur, 187 p.

- KAPEND M., voir BUSHABU M.M. (2002).
- KARENZI P.E. (1994), «Biomass in Rwanda», in HALL D. O. \& MAO Y. S. (eds.), Biomass Energy and Coal in Africa, Zed Books Ltd. in association with African Energy Policy Research NetworkAFREPEN, Gabarone, pp. 68-130.

- KARCH S., GARIN B., ASIDI N., MANZAMBI Z., SALAUN.J. \& MOUCHET J. (1993), «Moustiquaires imprégnées contre le paludisme au Zaïre», Annales de la Société belge de Médecine tropicale, 73, pp. 37-53.

- KASEBA M.K., PAEPE R., BOSKI T., HERBOSCH A. \& NAUD J. (1997), «Genèse et évolution des profils bauxitiques latéritiques sur des surfaces d'aplanissement post-éocènes au Bas-Zaïre (Zaïre)», Colloque international Cornet, Mons, 5-9 septembre 1994, Faculté Polytechnique de Mons, ARSOM, pp. 409-442.

- KASEREKA M., voir MUNYOLOLO Y. (1994).

- KAZADI E., voir GARIN B. (1993)

- KAYEMBE C.M.P., KAKESE K.B. \& SOLOTSHI M. (1993), "Caractéristiques socio-économiques des vendeurs des abords de quatre grands marchés de Lubumbashi: cas de Lusonga, Kenya, Kamalondo et Katuba \|», Bulletin Géographique de Kinshasa, GEOKIN, IV 1, pp. 25-36.

- KAYEMBE KALAMBAYI P. \& KIMPANGA DIANGA P. (2006), «Défis pour le traitement et le contrôle de la tuberculose en République démocratique du Congo", in DE DAPPER M. \& DE LAME D., Africa's Great Rift: Diversity and Unity, AfricaTervuren, ARSOM, pp. 239-247.

- KEBELA KEBELA P. (2004), «Santé pour tous: identification des facteurs explicatifs de l'accessibilité aux soins de santé dans la commune de Ngaba», Vivre et survivre à Kinshasa. Problématique du développement humain, pp. 49-84.

- KENNES E. (1997), «Du Zaïre à la R.D. du Congo: la vieille termitière terrassée par le vent de la savane», Afrika Focus, 13, 1-4, pp. 31-77.

- KENNES E., en coll. avec MUNKANA N'GE (2003), Essai biographique sur Laurent Désiré Kabila, Cahiers Africains, Afrika Studies, 57-58-59, Tervuren, Institut Africain-CEDAF, Paris, L'Harmattan, $436 \mathrm{p}$. 
- KENNES E., voir DE VILLERS G. (1998 et 2001).

- KERStiNG P. (2006), «Histoire Holocène des paysages du sud du Rwanda», Les Cahiers d'Outre-Mer, 235, pp. 399-412.

- KIKASSA F. (1995), "Chronique d'une Société civile en formation au Sud-Kivu», Zaïre-Afrique, 293, pp. 158-159.

- KIMONYO J.-P. (2008), Rwanda. Un génocide populaire, Paris, Karthala, Coll. Hommes et sociétés, 546 p.

- KIMPANGA DIANGA P., voir KAYEMBE KALAMBAYI P. (2006).

- KINGOMA MUNGANGA J.-P., voir BUKOME ITONGWA D. (2002).

- KINKELA SAVY \& MPANZU BALOMBA (2004), «Quid de la professionnalisation des "exploitants agricoles" à Kinshasa", Vivre et survivre à Kinshasa. Problématique du développement humain, pp. 85103.

- KLAUBER V., voir JEWSIEWICKI B. (2003).

- KNOCKAERT A. (2004), «A propos d'un ouvrage sur le Congo léopoldien. Les fantômes du Roi Léopold II de Adam Hochschild», Congo-Afrique, 381, pp. 4752.

- KNOCKAERT A. (2004), «A propos de la peinture populaire urbaine congolaise. I. Kin moto na Bruxelles. Quand Kinshasa réchauffe Bruxelles. II. Mami Wata. La peinture urbaine au Congo", CongoAfrique, 386, pp. 366-373.

- KÖNIG D. (1994), «Dégradation et érosion des sols au Rwanda», Bordeaux, Les Cahiers d'Outre-Mer, 185, pp. 35-47.

- KOYO J.-P. (2005), «L'aménagement durable des forêts tropicales face à la multiplicité des fonctions attendues des arbres et des écosystèmes forestiers: le cas du Basin du Congo", in DE DAPPER M., International Symposium. Tropical Forests in a Changing Global Context, pp. 19-38.

- KOUWENAAR A. (2004), «Le Fonds Monétaire international inclut la troisième Revue du programme Economique du Gouvernement», Congo-Afrique, 383, pp. 173-177.

- KUVIBIDILA S., YU L., ODE D., MBELE V. \& WARRIER R.J. (1993), «An epidemiological study of hemoglobin levels and prevalence of anemia in young children from Bas-Zaire», Annales de la Société belge de Médecine tropicale, 73, pp. 227-234

- La Compagnie de Jésus et l'Evangélisation en Afrique Centrale. Esprit, histoire et perspectives (1994), Centenaire de l'arrivée des Jésuites dans la Mission du Kwango 1893-1993, Actes du Colloque, Kimwenza, décembre 1993, Editions Loyola, Kinshasa, 384 p.

- LAGAE J. (2001), «In search of a "Comme chez soi". The ideal colonial house in Congo (1885-1960)", in VELLUT J.-L., Itinéraires croisés de la modernité. Congo belge (1920-1950), Cahiers Africains, 43-44, pp. 239-282 (28 photos, plans et dessins h.-t.).

- LAGAE J. (2007), «Léopoldville, Bruxelles: villes-miroirs? L'architecture et l'urbanisme d'une capitale coloniale et métropole africaine», in VELLUT J.-C. (dir.), Villes d'Afrique. Explorations en histoire urbaine, Cahiers Africains, 73, Africa-Tervuren, U.C.L., Paris, L'Harmattan, 31 fig. h.-t., pp. 67-99.

- LAGAE J. (2008a), "Kinshasa comme "chantier bruxellois". L'émergence d'une métropole "moderne" dans une colonie belge 1946-1960», Les Cahiers de la Fonderie, 38, Bruxelles et le Congo, pp. 24-31.

- LAGAE J. (2008b), «From "Patrimoine partagé" to "Whose Heritage"? Critical Reflections on Colonial Built Heritage in the City of Lubumbashi, Democratic Republic of the Congo", Afrika Focus, 21, 1, pp. 11-30.

- LAMBIN E. (1998), «Land-cover Dynamics in Africa. Observations by Remote Sensing and Spatial Modelling", Bull. ARSOM, 44, 4, pp. 645-656.

- LAMY E. \& DE CLERCK L. (éd.) (2004), L'ordre juridique colonial belge en Afrique centrale. Eléments d'histoire, Recueil d'études, Bruxelles, ARSOM, pp. 359-438.

- LANGENBERG V.T. (2008), On the limnology of Lake Tanganyika, Thesis Wageningen University, The Netherlands, $212 \mathrm{p}$.

- LANOTTE O. (2003), Guerres sans frontières en République démocratique du Congo, Bruxelles, Editions Complexe, GRIP, 264 p. 
- LARAQUE A. \& OLIVY J.-C. (1998), «Deux systèmes hydrologiques mitoyens mais opposés du Congo-Zaïre: la Cuvette congolaise et les plateaux Tékés», International Conference on Tropical Climatology, Meteorology and Hydrology, In Memoriam Franz Bultot, Brussels, May 1996, ARSOM, pp. 593-606.

- LAURENT P.-J. (1999), «Déstabilisation des paysanneries du Nord-Kivu: migrations, démocratisation et tenures», in MATHIEU P. \& WILLAME J.-C. (dir.), Conflits et guerres au Kivu et dans la région des Grands Lacs, Cahiers Africains, 39-40, pp. 63-83.

- LAURENT P.-J., voir MATHIEU P. (1999).

- Lauro A. (2005), Coloniaux, ménagères et prostituées au Congo belge (1885-1930), Bruxelles, Editions Labor-Histoire, $272 \mathrm{p}$.

- LAURO A., voir Bruxelles et le Congo (2008).

- LAVREAU J., voir TACK L. (2006).

- Le calleneC S., M'BOloko E. (1995), «L'évolution comparée des deux Congo, belge et français 1956-1965», in AGERON C.R. \& MICHEL M. (dir.), L'ère des décolonisations, sélection de textes $d u$ colloque "décolonisations comparées», Aix-en-Provence, 30 septembre3 octobre 1993, pp.209-222.

- LECLERCQ H. (1993), “L'économie populaire informelle à Kinshasa, approche macro-économique», ZaïreAfrique, 271, pp. 17-36.

- LECLERCQ H. (2001), «Le rôle économique du diamant dans le conflit congolais», in MONNIER L., JEWSIEWICKI B. \& DE VILLERS G. ( 2001), Chasse au diamant au Congo/Zaïre, Cahiers Africains, 45-46, pp. 47-78.

- LEDERER A. (1992). "La pêche maritime au Congo/Zaïre», Bull. ARSOM, 38, 2, pp. 303-343.

- LEDERER A. (1993), «Incident de frontière au Kivu», Mélanges Pierre Salmon II, Histoire et ethnologie africaines, édités par THOVERON G. \& LEGROS $\mathrm{H}$., Civilisations, $X L I, 1-2$, pp. 415-426.

- LEDERER A. (1995), "Le problème du "waterpest", Bull. ARSOM, 41, 2, pp. 235241.

- LEGROS H. (1995), «Le cheminement historique de l'identité des Yeke du Shaba
(Zaïre)», Bull. ARSOM, 41, 2, pp. 137-156. - LEGROS H. (1996a), Chasseurs d'ivoire. Une histoire du royaume yeke du Shaba (Zaïre), Editions de l'Université de Bruxelles, Institut de Sociologie, 259 p.

- LEGROS H. (1996b), «Les discours de la parenté. Idéologie politique et manipulations lignagères chez les Yeke du Shaba (Zaïre)", Cahiers d'Etudes Africaines, 143, pp.397-419.

- LEGROS H., voir DE MARET, 1993.

- LELO NZUZI (1992), "Gestion foncière et production de l'habitat urbain au Zaïre», GEOKIN, Bulletin géographique de Kinshasa, pp. 241-263.

- LELO NZUZI (1995), «L'apport du diamant artisanal à l'essor de l'économie urbaine informelle à Mbuji Mayi au Zaïre", RBG, 119, 1-2, pp. 161-173.

- LELO NZUZI (1999), "Les guerres au Congo-Kinshasa et la destruction du Parc National des Virunga», Acta Geographica, 120, IV, Paris, pp. 59-62.

- LELO NZUZI F. (2008), Kinshasa. Ville et Environnement, préface de Léon de Saint Moulin, Paris, L'Harmattan, $282 \mathrm{p}$.

- LELO NZUZI F., DICKENS E., LOKA KONGO \& MVUBU NTOTO (2004), "L'étude sur la situation socioéconomique des travailleurs navetteurs de la commune de Kisenso à Kinshasa», Vivre et survivre à Kinshasa. Problématique du développement humain, pp. 253288.

- LELO NZUZI F. \& TSHIMANGA MBUYI Cl. (2004) (dir.), Pauvreté urbaine à Kinshasa, Préface du Professeur Léon de Saint Moulin, La Haye, Cordaid, 167 p.

- LEMARCHAND R. (1996), Burundi: Ethnic Conflict and Genocide, Washington, Woodrow Wilson Center Press, 206 p. Ce livre avait été publié, en 1994, sous le titre: Burundi: ethnicide as discourse and practice.

- LEMARCHAND R. (2002a), «Le génocide de 1972 au Burundi: Les silences de l'Histoire», Cahiers d'Etudes Africaines, 167, XLII-3, pp. 551-567.

- LEMARCHAND R. (2002b), «A History of Genocide in Rwanda», Journal of African History, 43, pp. 307-311.

- LENOBLE-BART A. (1996), «Les enfants de la rue à Kigali: sortir de l'impasse?», Politique africaine, 63, pp. 72-78. 
- LE PAPE M (1997), «La presse et les réfugiés rwandais au Congo-Zaïre», $D u$ Zaïre au Congo, Afrique contemporaine, 183, pp. 57-66.

- LE ROY J., voir VAN WOLPUTTE S. (2002),

- LESH R.W., voir WILLIAM D.B. (1995).

- Les problèmes actuels de l'économie congolaise (2004), Congo-Afrique, 383, pp. 154-177.

- Les Temps Modernes (1995), Les politiques de la haine. Rwanda, Burundi 1994-1995, juillet-août, 583, 315 p.

- LETEINTURIER B. \& MALAISSE F. (1994), «De la réhabilitation des sites pollués par l'exploitation du cuivre en Afrique centro-australe», Bull. ARSOM, 45, 4, pp. 535-554.

- LETEINTURIER B. \& MALAISSE F. (1999), «The copper flora of Katanga: a phytogeographical analysis», GEO-ECOTROP, 23, pp. 31-48.

- LETEINTURIER B., voir MALAISSE F. (1997).

- LOKA KONGO, voir LELO NZUZI (2004).

- LONDA L., voir ILUNGA L. (1993), LELO NZUZI (2004).

- LORIAUX F., MORIMONT F. (sous la dir. de VELLUT J.-L.) (1996), Bibliographie historique du Zaïre à l'époque coloniale 1880-1960:Travaux publiés en 19601996. Centre d'histoire de l'Afrique, Coll. Enquêtes et documents d'histoire africaine, 14, Louvain-la Neuve, Tervuren, $\mathrm{V}+325 \mathrm{p}$.

- LORIS H. voir CHARLET J.M. (2005).

- LOUETTE M. (2006), «Data Mining of the Zoological Collections in the Royal Museum for Central Africa (RMCA), Tervuren, for Study and Conservation in the Albertine Rift», in DE DAPPER M. \& DE LAME D. (eds.), Africa's Great Rift: Diversity and Unity, Africa-Tervuren, ARSOM, pp. 73-81.

- LUBANA NGIYENZE AMENA (1992), «Processus de démocratisation et réalité socio-économiques du Zaïre en 1992. Le cas des projets de développement rural», Bulletin de Géographie de Kinshasa, GEOKIN, VII, 2, pp. 267-287.

- LUBOYA N., voir TELLIER V. (1996).

- lufuma kAPENDA A. (2000), «La répartition spatiale des antennes paraboliques à Lubumbashi (RD Congo). Premier inventaire et essai d'analyse socio-spatiale», Bull. Société Géographique de Liège, 39, 2, pp. 91-99.

- LUGAN B. (2007), Rwanda, Contreenquête sur le génocide, Toulouse, Privat, $330 \mathrm{p}$.

- LUISHYE TSHITALA E. (1993), «Les Kasaïens refoulés du Shaba à Kananga. Combien sont-ils? Comment sont-ils traités? Rapport sur la situation des refoulés kasaïens du Shaba arrivés à Kananga du 19 octobre 1992 au 28 août 1993», Zaïre-Afrique, 278, Kinshasa, pp. 511-514.

- LUKANDA M., voir BIZAMINA N.L. (1993)

- LUKESO P. (2005), «Et le chômage engendra le "sous-informel" ", CongoAfrique, 392, pp. 82-103.

- LUMBU B., voir BIZAMINA B.L. (1993).

- LUTUTALA B. (2006), “L'ubiquité résidentielle des migrants congolais. Une enquête auprès des migrants à Paris", in TREFON Th. \& PETIT P. (dir.), Expériences de recherche en République démocratique du Congo, Civilisations, LIV, 1-2, pp. 117-124.

- LUTUTALA MUMPASI (1997), «L'élargissement de l'espace de vie des Africains: comment le pays des "oncles" européens devient aussi celui des "neveux" africains», Revue Tiers Monde, 150, pp. 333346.

- LUYINDULA N., voir MAKUMBELO E. (2002 et 2005).

- LUZOLELE LOLA NKALALA, voir ERPICUM R. (1998).

- MABIALA MANTUBA NGOMA (dir.) (1999), Police et reconstruction nationale, Kinshasa, Publications de l'Institut pour la Démocratie et le Leadership politique, $154 \mathrm{p}$.

- MABIALA MANTUBA-NGOMA P. (dir.) (2004), La Nouvelle Histoire du Congo. Mélanges eurafricains offerts à Frans Bontinck, C.I.C.M., Cahiers Africains, 6566-67, Africa-Tervuren, Paris, L'Harmattan, $472 \mathrm{p}$.

- MABIALA MANTUMBA NGOMA (2007), «L'architecture chrétienne catholique de Kinshasa (1908-1988)», in VELLUT J.L. (dir.), Villes d'Afrique. Explorations en histoire urbaine, Cahiers Africains, 73, pp. 
101-112.

- MABOLOKO NGULAMBANGU C.E. \& MBENGA MPIEM LEY (1995), «Bandundu, une ville secondaire d'Afrique tropicale. Le rôle du contrôle étatique dans la production de l'espace», R.B.G., 119, 1-2, pp. 145-160.

- MABOLOKO NGULAMBANGU C.E. \& NICOLAÏ H. (1999), «Frontière, diamant et pauvreté. Le cas de la frontière CongoAngola au Kwango», R.B.G., 123, 4, pp. 265-275.

- MABIRA M., voir BUShABU M.-M. (2002).

- MAcGAFFEY J. (1995), «Chaotic Social Change in Zaire. Its Opportunities and problems for Entrepreneurs", in ELLIS S. \& FAURÉ Y.-A. (dir.), Entreprises et entrepreneurs africains, Paris, Karthala, ORSTOM, pp. 187-194.

- MAcGAFFEY J. \& BAZENGUISSAGANGA R. (2000), Congo-Paris: transnational traders on the margins of the law, Oxford, James Currey, Bloomington, Indian University Press.

- MAcGAFFEY W. (1993), «Anthropology of History", Liber Amicorum Marcel d'Hertefelt, Cahiers Africains, 4-5, Institut Africain-CEDAF, pp. 153-162.

- MACGAFFEY W. (2005), «Changing Representations in Central African History», Journal of African History, 46, pp. 189-207.

- MACMILLAN H. \& SHAPIRO F. (1999), Zion in Africa: the Jews of Zambia, London, I.B.Taurus.

- MAFIKIRI TSONGO A. (1994), La problématique foncière au Kivu montagneux (Zaïre), Cahiers du Cidep, Louvain-laNeuve, Academia, Paris, L'Harmattan, $181 \mathrm{p}$.

- MAFIKIRI TSONGO (1996), «Pratiques foncières, phénomènes informels et problèmes ethniques au Kivu (Zaïre)", in DE VILLERS G. (dir.), Phénomènes informels et dynamiques culturelles en Afrique, Cahiers Africains, 19-20, pp. 46-63.

- MAFIKIRI TSONGO A., voir MATHIEU P. (1999)

- MAHANGAIKO LEMBO E., voir BOURDOUX P. (2005)

- MAINDO M.N.A. (coord.) (2007), Des conflits locaux à la guerre régionale en Afrique Centrale. Le Congo-Kinshasa oriental 1996-2007, Paris, L'Harmattan, $310 \mathrm{p}$.

- MAKABU G., voir CHARLET J.M. (2000)

- MAKAMU NGANGULA MBELA (2002), "Charles TEBA, fonctionnaire de l'E.I.C. et du Congo belge à Boma (1901-1910?). Un des pionniers de l'intelligentsia congolaise", Congo-Afrique, 365, pp. 293-314.

- MAKAYA MBOKO MBENZA, voir D'ZOUZA (1995).

- MAKUMAYA NSAMBA KIAMBAMBA (1993), «Développement et problèmes spécifiques des infrastructures commerciales dans l'agglomération ndjiloise avant septembre 1991», Bulletin Géographique de Kinshasa GEOKIN, III, 2, pp. 217-240.

- MAKUMBELO E., PAULUS J.J. s.j., LUYINDULA N. \& LUKOKI L. (2002), «Inventaire des espèces végétales mises en culture dans les parcelles en milieu urbain. Cas de la commune de Limete, Kinshasa, République Démocratique du Congo», Tropicultura, 20, 2, pp. 89-95

- MAKUMBELO E., PAULUS J.J. s.j., LUYINDULA N. \& LUKOKI L. (2005), «Apport des arbres fruitiers à la sécurité alimentaire en milieu urbain tropical: cas de la commune de Limete-Kinshasa, République Démocratique du Congo», Tropicultura, 23, 4, pp. 245-252.

- MAKWALA ma MAVAMBU ye BEDA J. (1995), «Projets de développement et transformation du milieu rural au Zaïre. Limites des actions entreprises", Revue Belge de Géographie, 119, pp. 73-83.

- MAKWALA ma MAVAMBU ye BEDA J. (1999), «Démocratie et paix dans la société traditionnelle Kongo", in SABAKINU KIVILU (dir.), Démocratie et paix en République démocratique du Congo, Presses de l'Université de Kinshasa, pp. 11-26.

- MAKWALA ma MAVAMBU ye BEDA (dir.) (2000), Administration publique, outil du développement de la nation congolaise, Kinshasa, Publications de l'Institut pour la Démocratie et le Leadership politique, Konrad-Adenauer Stiftung, $166 \mathrm{p}$.

- MALAISSE F. (1994), «Cuivre et végétation au Shaba (Zaïre)», Bull. ARSOM, 40, 4, pp. 561-580.

- MALAISSE F. (1997), Se nourrir en forêt claire. Approche écologique et nutrition- 
nelle, Centre Technique de Coopération Agricole et Rurale CTA, Wageningen, Les Presses Agronomiques de Gembloux, 350 fig. et photos dont les trois quarts en couleurs, $384 \mathrm{p}$.

- MALAISSE F. (2002), «Campeophagy in Africa: a state of knowledge report", GEO-ECO-TROP, 26, 1, pp. 37-56.

- MALAISSE F. (2004), "Ressources alimentaires non conventionnelles», Tropicultura, numéro spécial du vingtième anniversaire, pp. 30-36.

- MALAISSE F., BAKER A.J.M. \& LETEINTURIER B. (1997), "Les espèces de Buchnera L. (Scrophulariaceae) des gisements cupro-cobaltifères du HautKatanga (République Démocratique du Congo)», GEO-ECO-TROP, 21, 1-4, pp. 51-64.

- MALAISSE F. \& PARENT G. (1997), «Chemical composition and energetic value of some edible products provided by hunting or gathering in the open forest (Miombo)», GEO-ECO-TROP, 21, 1-4, pp. 65-71.

- MALAISSE F., voir AMBE G.A. (1999), BOURDEAUX Q. (2003), LETEINTURIER B. (1994 et 1999), TSHIBANGU K. (1995, 1997).

- MALINGREAU J.-P., ACHARD F. \& MAYAUX P. (1998), Vegetation Map of Central Africa, Trees Tropical Ecosystem Environment Observation by Satellite, Joint Research Centre, Monitoring of Tropical Végetation Unit.

- MALINGREAU J.-P., voir MAYAUX P. (2000 a et b).

- MALOBA MAKANGA J.D., voir SAMBA G. (1999-2000).

- MAMDANI M. (2001), When Victims Become Killers: Colonialism, Nativism and Genocide in Rwanda, Princeton, N.J., Princeton Univ. Press, $X V I+364$ p. Ce livre traite surtout du cas des Banyarwanda du Congo, dans le Nord et le Sud-Kivu.

- MANDUNGU K., voir BIZAMINA B.L. (1993).

- MANKOTO S. \& MALDAGUE M. (2005), «Stratégie systémique appliquée à la gestion de la biodiversité. Cas de la Réserve de biosphère de Lukil (RDC)», in DE DAPPER M. (ed.), International Symposium. Tropical Forests in a Changing Global Context, pp. 115-126.
- MANZAMBI Z., voir KARCH S. (1993).

- MARCHAL J. (1996a), L'Etat libre du Congo: paradis perdu. L'histoire du Congo 1876-1900, 2 vol. II s'agit de l'édition française d'un livre publié en néerlandais en 1989.

- MARCHAL J. (1996b), E.D. Morel contre Léopold II. L'histoire du Congo 19001910, Paris, L'Harmattan (édition néerlandaise en 1985).

- MARCHAL J. (1999), Travail forcé pour le cuivre et pour l'or. L'histoire du Congo 1910-1945, tome 1.

- MARCHAL J. (2000), Travail forcé pour le rail. L'histoire du Congo 1910-1945, tome 2 .

- MARCHAL J. (2001), Travail forcé pour I'huile de palme de Lord Leverhulme, L'histoire du Congo 1910-1945, 395 p. Une édition anglaise en 2008, sous le titre, inspiré de celui du livre d'A. Hochschild (Leopold II's Ghost), Lord Leverhulme's ghosts: colonial exploitation in the Congo, Verso Books, $256 \mathrm{p}$, avec une introduction d'A. Hochschild.

- MARECHAL P. (2005) «La controverse sur Léopold II et le Congo dans la littérature et les médias", in VELLUT J.-L. et al. (dir.), La mémoire du Congo. Le temps colonial, Africa Tervuren, MRCA et Editions Snoeck, pp. 43-49.

- MARGUERAT Y. \& POITOU D. (éd.) (1994), À l'écoute des enfants de la rue en Afrique Noire, Paris, Fayard, 623 p.

- MARYSSE S., DE HERDT T., NDAYAMBAJE E. (1994), Rwanda. Appauvrissement et ajustements structurel, Cahiers Africains, 12, Bruxelles, Institut AfricainCEDAF, Paris, L'Harmattan, 86 p.

- MARYSSE S., NDAYAMBAJE E., WAETERLOOS E. (1993), Revenus ruraux au Rwanda avant l'ajustement structurel. Cas de Kirarambongo, Cahiers du Cidep, 19, Louvain-la-Neuve, Academia, Paris, L'Harmattan, $58 \mathrm{p}$.

- MARYSSE S. \& REYNTJENS F. (dir.), L'Afrique des Grands Lacs. Annuaire, Paris, L'Harmattan. Cet annuaire (de 300 à près de 500 pages) chevauche chaque fois deux années. Le premier, publié en 1997, est l'annuaire 1996-1997. Celui de 2005-2006, publié en 2006, porte le soustitre: Dix ans de transitions conflictuelles. - MARYSSE S., voir DE HERDT T. (1996), 
ZANA AZIZA ETAMBALA (1995).

- MASANGU MULAMGO J.-C. (2004), "La RDC accède au "point de décision" de l'Initiative en faveur des Pays pauvres très endettés», Congo-Afrique, 383, pp. 155-158.

- MASHINI DHI MBITA MULENGHE (1992), «Développement régional et stratégie spatiale. Quelles alternatives pour les espaces africains? L'exemple du Kwango-Kwilu (Sud-Ouest du Zaïre)», Bulletin géographique de Kinshasa GEOKIN, IV, 2, pp. 121-197.

- MASHINI DHI MBITA MULENGHE (1995), «Le rôle controversé de l'Etat au Zaïre et l'échec de la politique de décentralisation. L'exemple du Kwango-Kwilu», R.B.G., 119, pp. 135-144.

- MASHINI DHI MBITA MULENGHE (1998), «L'intégration socio-économique de la population originaire d'Afrique noire dans la région de Bruxelles-Capitale», R.B.G., 122, 1, pp. 55-70.

- MASHINI DHI MBITA MULENGHE, voir NICOLAÏ H. (1996), WOLFF E. (2001).

- MATHIEU P. \& MAFIKIRI TSONGO (1998), «Guerres paysannes au NordKivu 1937-1994», Cahiers d'Etudes africaines, 150-152, pp. 385-416.

- MATHIEU P. \& WILLAME J.C. (dir.), (1999), Conflits et guerres au Kivu et dans la région des Grands Lacs. Entre tensions locales et escalade régionale, Cahiers Africains, 39-40, Institut Africain-CEDAF, Tervuren, Paris, L'Harmattan, $218 \mathrm{p}$.

- MATANGilla A. (2006), «Pour une analyse du discours des Eglises de réveil à Kinshasa», in TREFON Th. \& PETIT P. (dir.), Expériences de recherche en République démocratique du Congo, Civilisations, LIV, 1-2, pp.77-84.

- MATHIEU P., LAURENT P.-J., MAFIKIRI TSONGO A., MUGANGU S. (1999), «Cohabitations imposées et tensions politiques au Nord-Kivu, 1939-1994: une trajectoire conflictuelle", in MATHIEU P. et WILLAME J.C. (dir.), Conflits et guerres au Kivu et dans la région des Grands Lacs. Cahiers Africains, 39-40, pp.13-20.

- MATHIEU P. \& MAFIKIRI TSONGO A. (1999), «Enjeux fonciers, déplacements de population et escalades conflictuelles (1930-1995)», in MATHIEU P. et WILLAME J.C. (dir.), Conflits et guerres au Kivu et dans la région des Grands Lacs. Cahiers Africains, 39-40, pp. 21- 62.

- MATHIEU P., MAFIIRI TSONGO A., MUGANGU MATABORO S. (1999), "Insécurisation et violence. Quelques réflexions sur les causes et remèdes possibles des escalades conflictuelles", in MATHIEU P. et WILLAME J.C. (dir.), Conflits et guerres au Kivu et dans la région des Grands Lacs. Cahiers Africains, 39-40, pp. 85-96.

- MAVANGA T., CIRIMWAMI M., MUHUGIRWA B., BAGALWA R. \& MUNDALA M. (1999), «Recrudescence des glissements de terrain suite à la réactivation sismique du bassin du lac Kivu, Région de Bukavu (République Démocratique du Congo)", MRCA, Département Géologie-Minéralogie, Rapport annuel 1997-1998, pp. 285-298.

- MAY J.P. (1995), «Policies on Population, Land Use and Environment in Rwanda», Population and Environment, 16, 4, pp. 321-334.

- MAY J. (1996), «Pression démographique et politiques de population au Rwanda 1962-1994», Population et Société, Bulletin mensuel d'information de I'Institut National d'Etudes Démographiques, 319, Paris, 4 p.

- MAYAUX P. et al. (1997), Carte de végétation de l'Afrique centrale à 1: 5000000 (bilingue français-anglais), Trees Tropical Ecosystems Environment Observation by Satellite, Joint Research Centre, European Commission, $35 \mathrm{p}$.

- MAYAUX P., DE GRANDI G. \& MALINGREAU J.-P. (2000a), "Central African Forest Cover Revisited: A Multisatellite Analysis», Remote Sensing Environment, 71, pp. 183-196.

- MAYAUX P. \& MALINGREAU J.-P. (2000b), «Le couvert forestier d'Afrique centrale: un nouvel état des lieux», Bull. ARSOM, 46, 4, pp. 475-486, 1 carte h.-t. en couleurs au 1/6000 000.

- MAYAUX P., voir MALINGREAU J.-P., (1998).

- MAYOYO BITUMBA TIPO TIPO (1995), Migration Sud/Nord. Levier ou obstacle? Les Zaïrois en Belgique, Cahiers africains, 13, Coll. Zaïre, années 90, volume 4, Bruxelles, Institut Africain-CEDAF, Paris, L'Harmattan, $167 \mathrm{p}$.

- MAYOYO BITUMBA TIPO-TIPO (2006), 
La Deuxième Guerre Occidentale contre le Congo. Offensive des médias et dessous des cartes, Espace Kinshasa, Paris, L'Harmattan, 283 p.

- MBARAGIJIMANA C. voir ILUNGA L. (2004).

- MBAYI R., voir SAMBA G. (1999-2000).

- MBELE V., voir KUVIBIDILA S. (1993).

- MBENGA MPIEM LEY, voir MABOLOKO (1995).

- MBENZA M., voir DIKUMBWA M. (1999).

- MEEUWIS M. (2005), «Het activisme van de verminking", FORUM, BVABA, 25, pp. 2-7, version française, «L'activisme de la mutilation», Ibid., pp. 8-12.

- MEEX P. (1997), «Historique du réseau triangulé au Congo belge/Zaïre», Bull. ARSOM, 43, pp. 193-215.

- MÉLICE A. (2001), «Le kimbanguisme: un millénarisme dynamique de la terre aux cieux», Millenarian Movements in Africa and the Diaspora, Bull. ARSOM, 47 (suppl.), in cooperation with Belgian Association of Africanists, pp. 35-54.

- MÉLICE A. (2006), «Un terrain fragmenté. Le kimbanguisme et ses ramifications», in TREFON Th. \& PETIT P. (dir.), Expériences de recherche en République démocratique du Congo, Civilisations, LIV, 1-2, pp. 67-76.

- MERCADER J., RUNGE F., VRYDAGHS L., DOUTRELEPONT $H_{\text {., }}$ EWANGO C.E.N., JUAN-TRESSERA J. (2000), «Phytoliths from archaeological sites in the tropical forest of Ituri, Democratic Republic of Congo", Quaternary Research, 54, 1, pp. 102-112.

- MERCADER J., ROVIRA S., GÓMEZRAMOS D. (2000), «Spared technologies: forager-farmer interaction and ancient iron metallurgy in the Ituri rain forest, D.R.C.», Azania, 35, pp. 107-122.

- MERCADER J. \& BROOKS A (1998), «Long-term settlement of lowland tropical rainforests, the evidence from Ituri, D.R. Congo", Journal of Human Evolution, 34, pp. A14-A15.

- MERCADER J. \& BROOKS A. (2001), «Across forests and savannahs. Later Stone assemblages from Semliki and Ituri, Democratic Republic of Congo», Journal of Anthropological Research, 57, pp. 197 217.
- MERCADER J., GARRALDA M.D., PEARSON O. \& BAILEY R. (2001), «EightHundred-Old Human Remains from the Ituri Tropical Forest, D.R.C.; the Rock Shelter Site of Matangai Turu Northwest», American Journal of Physical Anthropology, 115, pp. 24-37.

- MFUKALA MOKE KEY W.-R. (1994), «Stratégies matrimoniales et appropriation de la terre chez les Yansi. Pour une solidarité entre générations», Afrika Focus, 10, 1-2, pp. 81-105.

- MIAKALA M., voir ARBYN M. (1995).

- MIANDA D.M.G. (1995), «Dans l'ombre de la démocratie au Zaïre: la remise en question de l'émancipation mobutiste de la femme», Revue canadienne d'études africaines, 29, 1, pp. 51-78.

- MIANDA D.M.G. (1996), Femmes africaines et pouvoir. Les maraîchères de Kinshasa, préface de C. CoqueryVidrovitch, postface de B. Verhaegen, Collection Zaïre, Histoire et Société, Paris, L'Harmattan, 197 p.

- MICHA J.-C. \& GASHAGAZA J.-B. (2002), «Réussite de l'introduction du protoptère Protopterus aethiopicus Heckel dans le lac Muhazi (Rwanda)», Bull. ARSOM, 48, 3, pp. 315-218.

- MICHA J.C., voir KANANGIRE C.K. (2001), KANYINGINI B.(1999).

- MICHEL R. (1998), «Palaeoenvironmental Evidence for Synchronous Changes in Calcite Flowstones Formation, Speleothems Erosion and/or (Re)Dissolution and Equatorial Wetter Conditions in the Tropical Karst of Mount Hoyo (N.E.Zaire)", International Conference on Tropical Climatology, Meteorology and Hydrology, In Memoriam Franz Bultot, May 1996, Brussels, pp. 65-85.

- MICHIELSEN K., BOSMANS M., TEMMERMAN M. (2008), «Reducing HIV/AIDS in Young People in Sub-Sahara Africa: Gaps in Research and the Role of Theory", Afrika-Focus, 21, 1, pp. 31-43.

- MIDDLETON J. \& RASSAM A. (eds.) (1995), Encyclopaedia of world cultures, Volume IX, Africa and the Middle East, Boston, G.K. Hall.

- MIGEOTTE F. (1997), Une colline rwandaise à travers ses pratiques d'élevage, introduction de D. de Lame, Tervuren, MRCA, Annales Sciences 
humaines, vol. 160, $99 \mathrm{p}$.

- MIKOKORO C., voir SIMONARD T. (1994).

- MILY DENDA-SAKALA D. (1996), «Les sous-régions rurales de la Province Orientale dans la dynamique de l'histoire récente du Zaïre: de 1960 à 1985 (cas du Bas-Uélé)», Afrika Focus, 12, 4, pp. 191218.

- MINANI BIHUZO R. (2007), «La problématique des ressources minières en RDC: état des lieux et perspectives", CongoAfrique, 417, pp. 501-518.

- MINTEN B., voir GOOSSENS F (1994).

- MISSER F. \& VALLÉE O. (1997), Les gemmocraties: l'économie politique du diamant africain, Paris, Desclée de Brouwer, $243 \mathrm{p}$.

- MISSER F. \& VALLÉE O. (2001), «Du scandale zaïrois au Congo gemmocratique», in MONNIER L., JEWSIEWICKI B. \& DE VILLERS G. ( 2001), Chasse au diamant au Congo/Zaïre, Cahiers Africains, 45-46, pp. 27-46.

- MISSER F., voir CROS M.-F. (2006).

- MLIBWA WAZYE (1992), «Essai de classement régional des Zones rurales des régions du sud du Zaïre suivant certains critères de développement rural», Bulletin Géographique de Kinshasa, GEOKIN, III, 1, pp. 89-100.

- MOEYERSONS J. (1994), «Les essais récents de la lutte anti-érosive au Rwanda", Bordeaux, Les Cahiers d'OutreMer, 185, pp. 65-78.

- MOEYERSONS J. (2001), «The palaeoenvironmental significance of Late Pleistocene and Holocene creep and other geomorphic processes, Butare, Rwanda», Palaeoecology of Africa and Surrounding Islands, 27, pp. 37-50.

- MOEYERSONS J. (2003), «The topographic treshholds of hillslope incision in south-western Rwanda», Catena, 50, pp. 381-400.

- MOEYERSONS J., NYSSEN J., DECKERS J., MITIKU H. \& POESEN J. (2004), "The Climatic Significance of LatePleistocene and Early-to MiddleHolocene Mass Movements and their Present-day Remobilization in Rwanda and Ethiopia», in DEMARÉE G. et al., Second International Conference on Tropical Climatology, Meteorology and
Hydrology, ARSOM, pp. 63-79.

- MOEYERSONS J., voir SIBAMANA C. (1996).

- MOHYMONT B., voir DEMARÉE G. (2004).

- MOKILI J. (1998), Politiques agricoles et promotion rurale au Congo-Zaïre 18851997, Paris, L'Harmattan, 554 p.

- MOLIBA BANKANZA J.C. (2004), «Classification des régimes pluviométriques en République démocratique du Congo et en Angola», in DEMARÉE G. et al. (ed.), Second International Conference Tropical Climatology, Meteorology and Hydrology, ARSOM, pp. 295-305.

- MOLISHO D., voir BRUNEEL H. (1994). - MONNIER L., JEWSIEWICKI B. \& DE VILLERS G. (éd.) (2001), Chasse au diamant au Congo/Zaïre, Cahiers Africains, Afrika Studies, 45-46, Tervuren, Institut Africain-CEDAF, Paris, L'Harmattan, 240 p.

- MONNIER L., voir DE VILLERS G. (2002).

- MONSEMBULA J.-C. et al. (2005), "Gestion de la faune sauvage et commerce du gibier en Afrique centrale», in DE DAPPER M. (ed.), International Symposium. Tropical Forest in a Changing Global Context, ARSOM, pp. 282-292.

- MORIMONT F. (2001), «Les villes coloniales belges à travers L'lllustration congolaise», in VELLUT J.-L. (dir.), Itinéraires croisés de la modernité. Congo belge (1920-1950), Cahiers Africains, 43-44, pp. 24-56.

- MORIMONT F., voir LORIAUX F. (1996).

- MOSCOL-OliVEIRA M. \& ROCHE R. (1997), «Analyse palynologique d'une séquence sédimentaire holocène à Musisi-Karashoma (Kivu, R.D Congo). Influences climatiques et anthropiques sur l'environnement», GEO-ECO-TROP, $21(1-4)$, pp. 1-26.

- MOUCHET J., voir KARCH S. (1993).

- MOYA MAMONABWA H. (1993), «Le centenaire de la Paroisse de Kimwenza (1893-1993)», Zaïre-Afrique, 278, pp. 503-510.

- MPASI ZIWA MAMBU (1995), «Connaissances empiriques des mécanismes météorologiques, rites agraires et 
techniques agricoles du Bas-Zaïre. Une analyse d'ethnoclimatologie», RBG, 119, 1-2, pp. 85-95.

- MPAWENAYO B. (1996), Les eaux de la plaine de la Ruzizi (Burundi). Les milieux, la flore et la végétation algale, Bruxelles, ARSOM, Classe des Sciences naturelles et médicales, Mémoires, nouvelle série, tome 23, fasc. 2, $236 \mathrm{p}$.

- M'PENE NGALULEY Z. (2002), La problématique de l'intégration économique par les transports au Congo (R.D.C.), Paris, L'Harmattan, Louvain-la-Neuve, Bruylant Academia, 205 p.

- MPEY-NKA NGUB'USIM (1994), «Cette créativité qui fait survivre les jeunes de la rue à Kisangani», in MARGUERAT $Y$. et POITOU D. (éd.), À l'écoute des enfants de la rue en Afrique Noire, Paris, Fayard pp.491-507 (repris de Zaïre-Afrique, 215, 1987).

- MPURU M.B (1993), «Approvisionnement vivrier immédiat de Kikwit. Aperçu préliminaire», Bulletin Géographique de Kinshasa-Géokin, VI, 1, pp. 37-48.

- MUBIALA MUTOY (1997), «Les NationsUnies et la crise des réfugiés rwandais", Zaïre-Afrique, 313, pp. 133-156.

- MUCHEZ Ph. \& DEWAELE S. (2006), «Ore Deposits in the Neoproterozoic Rocks of the Katanga Province (Democratic Republic Congo). The Result of Multiple Mineralising Phases and Fluid Migrations», Bull. ARSOM, 52, 2, pp.185196.

- MUFOGOTO G. (2007), «Aux origines de l'actuel territoire d'Idiofa», CongoAfrique, 412-413, pp. 169-182.

- MUGIRANEZA A., voir ILUNGA L. (2006).

- MUGISHO E., DRAMAIX M., PORIGNON D., MUSUBAO E., HENNART P. (2002), «Evolution des données maternelles et périnatales recueillies en routine entre 1980 et 1998 à la maternité de référence de Rutshuru en République Démocratique du Congo. I. Décès maternels et interventions», Cahiers Santé, 12, pp. 245-251; «Il. Décès du nouveau-né et naissances de faible poids", Cahiers Santé, 12, pp. 252-255.

- MUGISHO SORO'GANE E., voir GOYENS Ph. (1996).

- MUHUGIRWA B., voir MAVANGA T. (1999).
- MUGIRANEZA A., voir ILUNGA L. (2006).

- MUHINDUKA DI-KURUBA (1993), «Aperçu de la politique économique zaïroise et perspectives», Zaïre-Afrique, 276, pp. 333-352.

- MUHIRE I. voir ILUNGA L. (2004).

- MUKADI KANKONDE et TOLLENS E. (éditeurs) (2001), Sécurité alimentaire au Congo-Kinshasa. Production, consommation et survie, Leuven, KULeuven, Paris, L'Harmattan, Collection Congo-Zaïre. Histoire et Société, 478 p.

- MUKAMBILWA K., voir MUNYOLOLO Y. (1999)

- MUKENZ-DIYAMBI KABN \& MNONGOMPASI A'NKUBA (1994), «La vie de sept jeunes filles de la rue à Kinshasa", in MARGUERAT Y. \& POITOU D. (éd.), À l'écoute des enfants de la rue en Afrique Noire, Paris, Fayard, pp. 398-404.

- MUKOSO NG'EKIEB F. (1993), Les origines et les débuts de la Mission du Kwango (1879-1914), Coll. Histoire du christianisme africain 1, C.A.E.K., Facultés Catholiques de Kinshasa, 319 p., 85 cartes et photos.

- MULINDA HABI BUGANZA (1993), "Aux origines du royaume de Ngoyo", Mélanges Pierre Salmon II, Civilisations, XLI, 1-2, pp. 165-187.

- MUMBANZA mWA BAWELE (1995), «L'évolution technologique des Bantu. Cas des riverains de la région équatoriale du Zaïre», Annales Aequatoria, 16, pp. 259-305.

- MUMBANZA mWA BAWELE (1997), «La pirogue dans l'ouest du bassin du Congo au milieu du 19 e siècle. Contribution à I'histoire de la navigation en Afrique précoloniale», Annales Aequatoria, 18, pp. 239-298.

- MUMBANZA mWA BAWELE (2003), «Villages entourés de fossés, abandonnés dans le Sud-Ubangi au milieu du 19e siècle? A quels peuples appartenaientils?», Annales Aequatoria, 24, pp. 53-76.

- MUMBANZA mWA BAWELE J.E. (2008), "Les sports et les loisirs à l'époque coloniale au Congo. Tradition et modernisme», in QUAEGHEBEUR M. \& BIBIANE TSHIBOLA (dir.), Aspects de la culture à l'époque coloniale en Afrique centrale, 9, Congo-Meuse, Paris, L'Har- 
mattan, pp. 226-251

- MUNDAL M., voir MAVANGA T. (1999).

- MUNKANA N'GE, voir KENNES E. (2003).

- MUNYOLOLO Y., WAFULA M., KASEREKA M., CIRABA M., MUKAMBILWA K., MUTUNDA MWEMBO (dir.) (1999), Justice et société en RDC, Kinshasa, Publications de l'Institut pour la Démocratie et le Leadership politique, $131 \mathrm{p}$.

- MUSUSA ULIMWENGU J. (1998), "Comment les Kinois perçoivent-ils la pauvreté?», Congo-Afrique, 329, pp. 523555.

- MUSUSA ULIMWENGU J., voir NGONGA NZINGA V (1999).

- MUTAMBA MAKOMBO KITATSHIMA J.M. (1998), Du Congo Belge au Congo Indépendant; 1940-1960. Emergence des «Evolués» et genèse du nationalisme, $688 \mathrm{p}$.

- MUTUMBA LUKUSA G. (1999), Congo/ Zaïre. La faillite d'un pays. Déséquilibre macro-économique et ajustements (19881999), Cahiers Africains, 37-38, Tervuren, Institut Africain-CEDAF, Paris, L'Harmattan, $190 \mathrm{p}$

- MUTUNDA MWEMBO (dir.) (1999), Justice et société en RDC, Kinshasa, Publications de l'Institut pour la Démocratie et le Leadership politique, $131 \mathrm{p}$.

- MUWALAWALA K. (1994), "Les formes d'oppression subies par les enfants de la rue à Kinshasa», in MARGUERAT Y. \& POITOU D. (éd.), À l'écoute des enfants de la rue en Afrique Noire, Paris, Fayard, pp. 361-375.

- MUZALIA WAKYEBWA K. (1993), «La distribution d'eau potable à Bukavu», in L'eau et l'aménagement dans l'Afrique des Grands Lacs, Colloque Bujumbura, 1991, pp. 193-216.

- MUZIGWA KASHEMA (1993), «À quand l'autosuffisance alimentaire du Zaïre? Une analyse comparative de l'assiette du Zaïrois par rapport à celle de quelques pays d'Afrique noire», Bulletin Géographique de Kinshasa, GEOKIN, IV, 1, pp. 93-106.

- MVUEMBA F., voir BIZIMANA B.L. (1993).

- MWANZA MA MWANZA (1995), «Kinshasa: stratégies et limites d'adapta- tion des transports urbains à la crise», R.B.G., 119, 1-2, pp. 123-134.

- MWANZA MA MWANZA (1996), «Transport et implantation des équipements socio-collectifs dans les métropoles africaines. Exemple de Kinshasa (Zaïre)», R.B.G., 129, 4, pp. 259-279.

- MWANZA ma MWANZA, (1997), Le transport urbain à Kinshasa. Un noeud gordien, Cahiers Africains, 30, Bruxelles, Institut Africain-CEDAF, Paris, L'Harmattan, 149 p. - MWANZA ma MWANZA (2000), «Choix des écoles et mobilité scolaire à Kinshasa», Bulletin de la Société Géographique de Liège, 39, pp. 79-89.

- MWANZA H. \& KABAMBA K. (2002), «Pauvreté et marginalisation rurales en Afrique au sud du Sahara», Belgeo, 1, pp. 3-16.

- MWENZE CHIRHU IWIRA NKINGI D. (1999), Bibliographie sur les Bashi du Sud-Kivu, Bibliothèque du centre d'Etudes des Religions Africaines, Facultés Catholiques de Kinshasa, 172 p. - MWOROHA E. \& CHRÉTIEN J.-P. (2003), «Le réseau princier des Baganwa au $X X e$ siècle. Du déclin politique à la nouvelle revendication de leur "ethnie" ", in PERROT Cl.-H. \& FAUVELLE-AYMAR F.-X. (dir.), Le retour des rois. Les autorités traditionnelles et l'Etat en Afrique contemporaine, Paris, Karthala, pp. 381-399.

- NAITHANI J., DELEERSNIJDER E., PLISNIER P.-D. \& LEGRAND S. (2004), «Preliminary Results of a Reduced-gravity Model of the Wind-induced Oscillations of the Thermocline in Lake Tanganyika», in DEMARÉE G., DE DAPPER M., ALEXANDRE J. (eds.), Second International Conference on Tropical Climatology, Meteorology and Hydrology, ARSOM, pp. 27-40.

- Nature et culture en République démocratique du Congo (2004), Tervuren, M.R.C.A, livre accompagnant l'exposition présentée au siège de l'Unesco, Paris, du 16 au 26 septembre 2004, au MRCA, Tervuren du 23 novembre 2004 au 2 octobre 2005, 120 p. II existe une édition néerlandaise et une version anglaise.

- NAUD J., voir KASEBA M.K. (1997).

- NDARISHIKANYE B (1998), «Le rapport Etat-paysannerie au centre du conflit eth- 
nique au Burundi», Cahiers d'Etudes africaines, 150-152, pp. 331-345.

- NDAYAMBAJE E., voir MARYSSE F. (1993 et 1994).

- NDAYIRAGUE G. (1993), «L'aménagement hydroélectrique dans la région des Grands Lacs", L'eau et l'aménagement dans l'Afrique des Grands Lacs, Colloque Bujumbura, 1991, pp. 323-342.

- NDAYIRUKIYE S. (1993), «La pêche artisanale dans le lac Tanganyika», Colloque 1991 Bujumbura, Collection Pays enclavés, Talence, CRET, n5, pp. 259-278.

- NDAYIRUKIYE S. (1994), «Evolution des paysages et développement agricole au Buyogoma», in GAHAMA J. \& THIBON C. (dir.) (1994), Les régions orientales du Burundi. Une périphérie à l'épreuve du développement, Paris, Karthala, pp. 71112.

- NDAYISIMIYE J.-P. (1993), «L'eau et la santé en milieu rural au Burundi», L'eau et l'aménagement dans l'Afrique des Grands Lacs, Colloque Bujumbura, 1991, pp. 146-158.

- NDAYWEL È NZIEM I. (1993), La société zaïroise dans le miroir de son discours religieux (1990-1993), Bruxelles, Institut Africain, Paris, L'Harmattan, 102 p.

- NDAYWEL È NZIEM I. (1994), «Importance et état du patrimoine scientifique et culturel de la Belgique concernant le Zaïre. Réflexions complémentaires», in DE VILLERS G. (dir.), Belgique-Zaïre. Une histoire en quête d'avenir, pp. 145-151.

- NDAYWEL È NZIEM I. (1995), «La littérature scientifique des années 90 sur le Zaïre. Eléments pour une historiographie du Zaïre postcolonial», Zaïre-Afrique, 299, Kinshasa, pp. 555-566.

- NDAYWEL È NZIEM I. (1996), «Le Zaïre de demain: identités et symboles, ZaïreAfrique, 303, pp. 78-98.

- NDAYWEL È NZIEM I (1997), Histoire du Zaïre. De l'héritage ancien à l'âge contemporain, Préface de T. Obenga, Postface de P. Salmon, Louvain-la-Neuve, Duculot, Afrique Editions, Agence de la Francophonie, 918 p. Une deuxième édition, en 1998, a été publiée sous le titre Histoire générale du Congo. De l'héritage ancien à la République démocratique, Paris, Bruxelles, De Boeck et Larcier, 955 p.
- NDAYWEL È NZIEM I. (1998), «Du Congo des rébellions au Zaïre des pillages", in Disciplines et déchirures. Les formes de la violence, Cahiers d'Etudes africaines, 150-152, pp. 417-439.

- NDAYWEL È NZIEM I. (2002), «Le milieu médical à l'épreuve de l'informel. "Survivre" comme infirmière aux Cliniques universitaires de Kinshasa", in DE VILLERS G., JEWSIEWICKI B. \& MONNIER L. (éd.) (2002), Manières de vivre: économie de la "débrouille» dans les villes du Congo/Zaïre, Cahiers africains, 49-50, pp. 141- 169.

- NDAYWEL È NZIEM I. (2004), «La nationalité congolaise à l'épreuve du séisme des Grands Lacs», in MABIALA MANTUBA-NGOMA P. (dir.), La Nouvelle Histoire du Congo, Cahiers Africains, 6566-67, pp. 407-427.

- NDAYWEL È NZIEM I., voir MUNYOLOLO Y. (1994).

- NEWBURY D. (1993), «Rwandan chronology in regional perspective. A reconsideration of the Ruganzu cycle», Liber Amicorum Marcel d'Hertefelt, Cahiers Africains, 4-5, pp. 163-208.

- NEWBURY D. (1997), «Irredentist Rwanda: ethnic and territorial frontiers in Central Africa», Africa Today, 44, 2, pp. 211-221.

- NEWBURY C. \& NEWBURY D. (2000), «Bringing the Peasants Back», in Agrarian Themes in the Construction and Corrosion of Statist Historiography in Rwanda, American Historical Review, CV, 3, pp. 831-877.

- NEWELL E. (1995), «Les foyers d'onchocercose au Burundi: leur étendue et la population à risque», Annales de la Société belge de Médecine tropicale, 35, pp. 273-281.

- NGBWAPKWA TE MOBUSA (2003), «L'exploitation du caoutchouc par l'Etat Indépendant du Congo dans le territoire de Banzyville, district de l'Ubangi (19001908)", Mélanges Pierre Salmon, II, Civilisations, XLI, 1-2, pp. 291-306.

- NGONDO A PITSHANDENGE S. (1995), «Le recensement pré-électoral, l'identification et l'enrôlement de la population dans le contexte zaïrois. Quelques problèmes pratiques», Zaïre-Afrique, 292, pp. 105-123. 
- NGONDO A PITSHANDENGE S. (1996), «Nucléarisation du ménage biologique et renforcement du ménage social à Kinshasa. Les retombées d'une transition démographique de crise», Zaïre-Afrique, 308, pp. 419-444.

- NGONDO A PITSHANDENGE S. (2000a), «De la mortalité maternelle en République Démocratique du Congo à l'aube du $3^{e}$ millénaire. Niveaux et facteurs explicatifs", Congo-Afrique, 347, pp. $410-436$

- NGONDO A PITSHANDENGE S. (2000b), «Kikwit: les problèmes de développement d'une ville régionale», Congo-Afrique, 342, pp. 80-101.

- NGONDO A PITSHANDENGE S. (2001), «Du vieillissement de la population en RDC: état de la question, mécanismes explicatifs, facteurs de promotion et propositions de stratégies», CongoAfrique, 358, pp. 488-505.

- NGONGA NZINGA V. (2004), «Bilan du Programme Intérimaire Renforcé et évaluation à mi-parcours du Programme Economique du Gouvernement (PEG)», Congo-Afrique, 383, pp. 159-168; «Le Programme Economique du Gouvernement (PEG)», pp. 169-173.

- NGOY B., voir TREFON Th. (2007).

- NGWABIJE R. (1994), «Le paysan rwandais et la problématique du développement agricole et rural: hiérarchisation des contraintes et esquisse des voies de solution», Bull. ARSOM, 40, suppl. 1, pp. 35-65.

- NGONGA NZINGA V. \& MUSUSA ULIMWENGU J. (1999), «Persistance de la dollarisation au Congo-Kinshasa: manifestation de la complémentarité et/ou de la substitution monétaire?», Cahiers Economiques et Sociaux, XXV, 1, IRESUniversité de Kinshasa.

- NGUNGU KASAI, D. P. (2004), «Analyse des mécanismes et stratégies de survie de la population de Kinshasa: "cas des boutiques et liglabos"», Vivre et survivre à Kinshasa. Problématique du développement humain, pp. 11- 48.

- NGWABIJE R. (1994), «Le paysan rwandais et la problématique du développement agricole et rural: hiérarchisation des contraintes et esquisse des voies de solution", Bull. ARSOM, 40,
Suppl. n¹, pp. 35-65.

- NGUB'USIM MPEG-NKA R. (2002), «Les années plastiques et le cycle triennal des années blanches à l'Université de Kinshasa de 1990 à 2001", CongoAfrique, 363, pp. 158-181.

- NICOLAï H., (1993a), «Progrès de la connaissance géographique du Zaïre, du Rwanda et du Burundi. Vingtième article. De 1989 à 1992", Bull. SOBEG, XLI, 2, pp. 235-306.

- NICOLAï H. (1993b), «Le Mouvement géographique. Un journal et un géographe au service de la colonisation du Congo", Mélanges Pierre Salmon (édités par THOVERON G. et LEGROS $\mathrm{H}$.), Civilisations, XLI, 1-2, pp. 257-277.

- NICOLAÏ H. (1994a), «Les géographes belges et le Congo", in BRUNEAU $\mathrm{M}$. et DORY D. (dir.), Géographie des colonisations XVe-XXe siècles, Paris, L'Harmattan, coll. Géotextes, pp. 51-65.

- NICOLAï H. (1994b), «Réflexions sur la ville africaine comparée à la ville européenne», Liber Amicorum Herman Van der Haegen, Acta Geographica Lovaniensa, 34, Heverlee, Louvain-laNeuve, pp. 479-488.

- NICOLAÏ H. (1995), «Les réseaux urbains de l'Afrique tropicale: origines, évolutions et tendances", in NZISABIRA J., Organisation de l'espace: le système dominant et son fonctionnement, Coll. Population et développement, $n^{\circ} 2$, Louvain-la-Neuve, Academia-Bruylant, Paris, L'Harmattan, annexe 1, pp.125147.

- NICOLAï H. (1996a), «Réflexions sur les caractères originaux des paysages agraires de l'Afrique tropicale", in DONNAY J.-P. \& CHEVIGNÉ Cl. (éd.), Hommage au Professeur Charles Christians, Société Géographique de Liège, numéro hors-série, pp. 316-319.

- NICOLAï H. (1996b), «Les transformations d'un espace africain: le Kwilu», SOBEG, pp. 15-34.

- NICOLAï H. (1997), «Composantes et implications démographiques du drame rwandais», Rwanda-Burundi. Journée d'étude, Bruxelles, 10 janvier 1997, ARSOM, pp. 11-33.

- NICOLAï H. (1998a), La répartition et la densité de la population au Kivu, 
Bruxelles, ARSOM, Mémoires in- $8^{\circ}$, Nouvelle série, Tome 24, fasc. 2, 64 p., 2 cartes h.-t. en couleurs.

- NICOLAï H. (1998b), «Geografia, popolazione e violenza nelle regione dei Grandi Laghi», Terra d'Africa, VII, Edizioni Unicopoli, Milano, pp.17-56.

- NICOLAï H. (2001), «La géographie», in HALLEUX R. et al. (dir. scientifique), Histoire des sciences en Belgique 1815 2000, 2, ch. 14, Les sciences d'outre-mer, La Renaissance du Livre, Dexia, pp. 247250.

- NICOLAï H. (2005), «L'image du Congo par la carte. De la boussole et de la planchette à l'avion», in VELLUT J.-L. et al., La mémoire du Congo. Le temps colonial, MRCA, Africa Tervuren, Editions Snoeck, 2005, pp. 139-149.

- NICOLAÏ H. (2007), «La géographie, les géographes et le Congo", Mondes et cultures, compte rendu annuel des travaux de l'Académie des Sciences d'Outre-Mer, Paris, tome LXVII, vol.1, pp. 337-349.

- NICOLAÏ H., GOUROU P., MASHINI DHI MBITA MULENGHE (1996), L'espace zaïrois. Hommes et milieux, Collection Zaïre. Histoire et Société, Bruxelles, Institut Africain-CEDAF, Paris, L'Harmattan, 607 p.

- NICOLAÏ H., voir MABOLOKO (1999).

- NKUKU KHONDE RÉMON M. (2006), Stratégies de survie à Lubumbashi (R.D. Congo). Enquête sur 14000 ménages urbains, Coll. Mémoires lieux de savoir. Archive congolaise, Paris, L'Harmattan, $131 \mathrm{p}$.

- NKURUNZIZA F.-X. (1996), «Analyse des principales contraintes de développement des régions de montagnes du Burundi», in DONNAY J.-P., CHEVIGNÉ Cl. (éd. scientifiques), Recherches de géographie humaine. Hommage au Professeur Charles Christians, Société Géographique de Liège, $\mathrm{n}^{\circ}$ hors-série, pp. 321-326.

- NOTERMAN J.-P., voir PORIGNON D. (1995).

- NOTI N'SELE ZOZE (1992), «Réflexions méthodologiques sur l'étude de mutations géographiques et crise de développement dans les régions riveraines du BasKasai (Zaïre)», Bulletin Géographique de Kinshasa, GEOKIN, III, 1, pp. 59-75.

- NSABIMANA T. (1993a), “L'aménage- ment des bas-fonds et marais du Burundi: une approche historique", L'eau et l'aménagement dans l'Afrique des Grands Lacs, Colloque Bujumbura 1991, Talence, CRET, pp. 92-103.

- NSABIMANA S. (1993b), «L'adduction d'eau potable en milieu rural au Burundi», L'eau et l'aménagement dans l'Afrique des Grands Lacs, Colloque Bujumbura, 1991, pp. 158-175.

- NSABIMANA T. \& MANIRAKISA J.-B. (1994), "Les enjeux de l'exploitation des marais», in GAHAMA J. \& THIBON C. (dir.) (1994), Les régions orientales du Burundi. Une périphérie à l'épreuve du développement, Paris, Karthala, pp. 137146.

- N'SANDA BULELI L. (2005), Ethnicité et "géopolitique" au Maniema (R.D. Congo), Collection Mémoires, lieux de savoir, Paris, L'Harmattan, $306 \mathrm{p}$.

- NTAGAND Ch., voir ROCHE E. (1998).

- N'TOTO M'VUBU (2001), «Budget de consommation des ménages: structure et déterminants. Cas de quelques quartiers pauvres de la ville de Kinshasa: Kisenso, Kindele et Makala», in KANKONDE MUKADI \& TOLLENS E., Sécurité alimentaire au Congo-Kinshasa. Production, consommation et survie, KULeuven, Paris, L'Harmattan, pp. 365-405.

- NTUMBA K. (1999), «Marchés ruraux et relations ville-campagne dans l'arrièrepays immédiat de Kananga (Congo)», Bull. Société Géographique de Liège, 36, pp. 93-101.

- NTUMBA LUABA LUMU (1999), «Grandes puissances et avenir de la République Démocratique du Congo", in SABAKINU KIVILU (dir.), Démocratie et paix en République Démocratique du Congo, Kinshasa, Presses de l'Université de Kinshasa, pp. 219-240.

- NYOKA MUPANGILA, voir KABAMBA KABATA (1998).

- NYORI I. A., BUGA B.L. (1996), «Surpopulation et environnement en Ituri (région du Haut-Zaïre, Zaïre)», Revue de géographie du Cameroun, 12, 2, pp. 1526.

- NZAMWITA D. (1993), «La maîtrise de l'eau sur le bassin-versant de la Kinoni (Rwanda)», L'eau et l'aménagement dans l'Afrique des Grands Lacs, Colloque 
Bujumbura 1991, pp. 21-42.

- NZEZA KABU ZEY-KONGO J.-P. (1999), «Le problème de l'approvisionnement de Kinshasa en viande locale», Les Cahiers d'Outre-Mer, LII, 206, pp.169-196.

- NZILAMBI NZILA E. et al. (1996), Profil des prostituées de Kinshasa, Zaïre, en 1994, Kinshasa, Centre M.T.S., 31 p. Un compte rendu a été publié par MUYENDO MILEMBE S. (1997), dans Zaïre-Afrique, 312, pp. 182-184

- NZONGOLA-NTALAJA G. (2003), The Congo from Leopold to Kabila: A people's history, London and New York, Zed Books, $304 \mathrm{p}$.

- OBOTELA R. N. (1997), «Le projet de société de la IIIe République à l'expérience des incertitudes politiques, démographiques et sociales», Zaïre-Afrique, 314, pp. 197-229.

- OBOTELA RASHIDI N. (2004), «La problématique des langues nationales en République démocratique du Congo ou l'histoire à l'épreuve des clivages socioculturels», in MABIALA MANTUBANGOMA P., La Nouvelle Histoire du Congo. Mélanges eurafricains offerts à Frans Bontinck, C.I.C.M., Cahiers Africains, 65-66-67, pp. 201-208

- ODE D., voir KUVIBIDILA S. (1993)

- OLIVY J.-C., voir LARAQUE A. (1998).

- OLLEVIER F., voir VERHEUST L. (1995).

- OLSON J. (1995), «Behind the recent tragedy in Rwanda», Questioning development. Growth? Destruction? Sustainabilty? Geojournal, 35, 2, pp. 217-222.

- OMASOMBO TSHONDA J. (2001), «Les diamants de Kisangani: de nouveaux seigneurs se taillent des fiefs sur le modèle de l'état zaïrois de Mobutu», in MONNIER L., JEWSIEWICKI B. \& DE VILLERS G. (2001), Chasse au diamant au Congo/Zaïre, Cahiers Africains, 45-46, Tervuren, Institut Africain-CEDAF, Paris, L'Harmattan, pp. 79-126.

- OMASOMBO TSHONDA J. (2002), «Vivre à Kisangani. Le cas de l'économie du transport», in DE VILLERS G., JEWSIEWICKI B. \& MONNIER L. (éd.) (2002), Manières de vivre: économie de la "débrouille" dans les villes du Congo/ Zaïre, Cahiers africains, 49-50, pp. 91111.

- OMASOMBO TSHONDA J. \& VERHAE-
GEN B. (1998), Patrice Lumumba. Jeunesse et Apprentissage Politique 1925-1956, Cahiers Africains, 33-34, Tervuren, Institut Africain-CEDAF, Paris, L'Harmattan, 265-p.

- OMASOMBO J. \& VERHAEGEN B. (2005), Patrice Lumumba, acteur politique. De la prison aux portes du pouvoir (juillet 1956-février 1960), Cahiers africains, 68-70, Africa-Tervuren, Paris, L'Harmattan, $405 \mathrm{p}$.

- OMASOMBO TSHONDA J., voir DE VILLERS G. (1997, 1998, 2001), VERHAEGEN B. (2006).

- PAELINCK H. (2004), «Sustainable Timber Production in the Democratic Republic Congo? Lessons from a Succesful Experience in the 1980s", in DE DAPPER M. (ed.), Tropical Forests: a State of the Art at the Turn of the Century, ARSOM, pp. 113-118.

- PAEPE R., voir KASEBA M.K. (1997), VAN OVERLOOP E. (1998).

- PAGÉZY H. (1995), «De l'adaptation nutritionnelle à la malnutrition: interactions écologiques et socio-culturelles dans l'alimentation des Ntomba du Zaïre», in DEVISCH R., DE BOECK F., JONCKERS D. (dir.) (1995), Alimentation, traditions et développement en Afrique intertropicale, Paris, L'Harmattan, pp. 272-303.

- PAGÉZY H. (1996), «Aspects psychoculturels de l'exploitation des ressources naturelles dans la région du lac Tumba (Zaïre)», in FROMENT A., de GARINE J., BINANA BIKOY C. \& LOUNGE J.F. (éd.), Bien manger et bien vivre. Anthropologie alimentaire et développement en Afrique intertropicale: du biologique au social, Paris, L'Harmattan, pp. 447-458.

- PAGÉZY H. (2006), «Le contexte magico-religieux de la pêche au lac Tumba: entre le "normal" et l'insolite», Journal des africanistes, 76, 2, pp. 44-62.

- PAJUEN H. (1998), «Peat accumulation Rate in Rwanda and Burundi. An Indication of Long-term Climatic Changes?», in DE MARÉE G. et al., International Conference on Climatology, Meteorology and Hydrology, In Memoriam Franz Bultot, Brussels, May 1996, ARSOM, pp.128-141.

- PARENT G., voir MALAISSE F. (1997).

- PAULUS J.J., voir MAKUMBELO (2002 
et 2005).

- PEEMANS J.-P. (1997), Crise de la modernisation et pratiques populaires au Congo-Zaïre. La quête démocratique, Coll. Point de vue, Paris, L'Harmattan, 206 p.

- PEEMANS J.-P. (1998), Le Congo-Zaïre au gré du XXe siècle. Etat, économie, société 1880-1990, préface de B. Verhaegen, Coll. Zaïre, Histoire et société, Paris, L'Harmattan, 286 p.

- PENDJE G. (1993), «Croissance et productivité de deux essences forestières plantées au Mayumbe, Zaïre: le Limba (Terminalia superba Engl. et Diels) et le Bilinga (Nauclea diderrichi (De Wild.) Merrill)», GEO-ECO-TROP, 17, 1-4, pp. 101-120.

- PÉROUSE dE MONTCLOS M.-A. (2000), Kigali après la guerre: la question foncière et l'accès au logement, Paris, Les Dossiers du CEPED, 57, 36 p.

- PETIT P. (1996a), «Au coeur du royaume. Réflexions sur l'ethnicité luba, Bulletin ARSOM, 42, 4, pp. 759-774.

- PETIT P. (1996b), «"Les charmes du roi sont les esprits des morts": les fondements religieux de la royauté sacrée chez les Luba du Zaïre», Africa, 66, 3, pp. 349366.

- PETIT P. (2000), Les sauniers de la savane orientale. Approche ethnographique de l'industrie du sel chez les Luba, Bemba et populations apparentées (Congo, Zambie), Bruxelles, ARSOM, Classe des Sciences morales et politiques, Mémoire in- $8^{\circ}$, Nouvelle série, Tome 52, fascicule 4, $141 \mathrm{p}$.

- PETIT P. (éd.) (2001), Lubumbashi 2000 : la situation des ménages dans une économie de précarité. Rapport des recherches effectuées durant la première session des travaux de l'Observatoire du changement urbain, juin-octobre 2000, $195 \mathrm{p}$.

- PETIT P. (2002), «Lubumbashi au présent: précarité et reconstruction dans la "crise" congolaise», Bull. ARSOM, 48, 2, pp. 123-141.

- PETIT P. (dir.) (2003), Ménages de Lubumbashi, entre précarité et recomposition, Paris, L'Harmattan, VI + 294 p.

- PETIT P. (dir.) (2004), Byakula: approche socio-anthropologique de l'ali- mentation à Lubumbashi, Bruxelles, ARSOM, $366 \mathrm{p}$.

- PETIT P., voir TREFON Th. (2006).

- PETRIDIS C. (2000), «Beknopte aantekeningen over een luipaardgenootschap en een rammasker bij de Luntu», Bull. ARSOM, 46, 2, pp. 113-132.

- PEYROT B. (1997), «Dynamiques paléoécologique et anthropogène de la forêt ombrophile de la dorsale Congo-Nil au Burundi», Les Cahiers d'Outre-Mer, 199, Bordeaux, pp. 271-292.

- PIERMAY J.L. (1993), «L'article 15, ou le Zaïre à la recherche d'articulations de rechange», Espaces africains en crise: formes d'adaptation et de réorganisation, Travaux de l'Institut de géographie de Reims, 83-84, pp. 94-107.

- PIROTTE G., PONCELET M. (2002), «Eveil des société civiles en milieu urbain et organisations non gouvernementales: les exemples de Cotonou et Lubumbashi », Autrepart, 23, pp. 73-88.

- PLISSART M.-F., voir DE BOECK F. (2005).

- PLISNIER P.-D. (1998), «Lake Tanganyika: recent Climate Changes and Teleconnections with ENSO», in DEMARÉE G. et al., International Conference on Tropical Climatology, Meteorology and Hydrology, In Memoriam Franz Bultot, Brussels, May 1996, ARSOM, pp. 228250.

- PLISNIER P.-D. (2004), «Probable Impact of Global Warming and ENSO on Lake Tanganyika» Bull. ARSOM, 50, 2, pp. 185-196.

- POITOU D., voir MARGUERAT Y. (1994).

- PONCELET M. (2001), «Les sciences sociales», in HALLEUX R. et al., Histoire des sciences en Belgique 1815-2000, 2, ch. 14, Les sciences d'outre-mer, Bruxelles, La Renaissance du Livre, Dexia, pp. 235-246.

- PONCELET M., PIROTTE G., STANGHERLIN G. \& SINDAYIHEBUGA E. (2006), Les ONG en ville africaine. Etude de cas à Cotonou (Bénin) et Lubumbashi (RDC), Louvain-la-Neuve, Academia Bruylant, $191 \mathrm{p}$.

- PONCELET M. (2008), L'invention des sciences coloniales belges, Coll. Homme et Société-Anthropologie, Paris, Karthala, $420 \mathrm{p}$. 
- PONCELET M., voir PIROTTE G. (2002). - PORIGNON D., NOTERMAN J.-P., HENNART Ph., TONGLET R., MUGISHO SORON'GANE E., ELONGO LOKOMBE T. (1995), "The Role of the Zairian Health Services in the Rwandan Refugee Crisis", Disasters, 19, 4, pp. 356-360.

- PORIGNON D., MUGISHO SORON'GANE E., ELONGO LOKOMBE T., KATULANYA ISU D., HENNART Ph., VAN LERGERGHE W. (1998), «How robust are district health systems? Coping with crisis and disasters in Rutshuru in Democratic Republic of Congo", Tropical Medicine and International Health, 7, pp. 559-565. - PORIGNON D., HENNART P., DRAMAIX M., DONNEN P. (2006), «Méthodologie de la recherche en santé: systèmes, savoirs et acteurs en République démocratique du Congo", Civilisations, LIV, 1-2, pp. 167-178.

- PORIGNON D., KAHINDO J.-B., PALUKU J.-L., DONNEN Ph., DRAMAIX M. \& HENNART Ph. (2006), «Health Research in Eastern Congo and Rwanda: A Tool for Improving Health System's Resilience and Peace-building Process", in DE DAPPER M. \& DE LAME D. (eds.), Africa's Great Rift: Diversity and Unity, Africa-Tervuren ARSOM, pp. 263-272.

- PORIGNON D., voir HENNART Ph. (1996).

- PORIGNON D., voir GOYENS Ph. (1996).

- POURTIER R. (1993), «Désorganisation des transports et spirale du sousdéveloppement au Zaïre», Politiques agricoles et initiatives locales, ORSTOM, Colloques et séminaires, pp. 49-69.

- POURTIER R. (1996), «La guerre au Kivu: un conflit multidimensionnel», Afrique contemporaine, 180, pp. 15-38.

- POURTIER R. (1997a), «Du Zaïre au Congo: un territoire en quête d'Etat», $D u$ Zaïre au Congo, Afrique contemporaine, 183, pp. 7-30.

- POURTIER R. (1997b), «Congo-ZaïreCongo: un itinéraire géopolitique au coeur de l'Afrique», Géopolitique d'une Afrique médiane, Hérodote, 86-87, pp. 6-41.

- POURTIER R. (1998), «Les refoulés du Zaïre identité, autochtonie et enjeux politiques», in LASSAILLY-JACOB V. (éd.), Communautés déracinées dans les pays du Sud, Autrepart, Cahiers des sciences humaines, nouvelle série, $\mathrm{n}^{\circ} 5$, ORSTOM, pp. 137-154.

- POURTIER R. (2002), «Le Congo (RDC) entre guerre et pillage», Géographies, Bulletin de l'Association de Géographes Français, 79, 2, pp. 251-263.

- POURTIER R. (2003), «L'Afrique centrale dans la tourmente: les enjeux de la guerre et de la paix au Congo et alentour», Tragédies africaines, Hérodote, 111, pp.11-40.

- POURTIER R. (2006), «Les réfugiés en Afrique centrale: une approche géopolitique», Géographies, Bull. Association de Géographes Français, pp. 50-61.

- POURTIER R. (2007), «Les chemins de fer en Afrique subsaharienne, entre passé révolu et recompositions incertaines", Belgeo, 2, pp.189-202.

- PRIOUL C. (1995), «Collines de Turquoise», Les Cahiers nantais, 44-45, pp. 253-268.

- PRUNIER G. (1995), The Rwanda crisis, 1959-1994: history of a genocide, London, Hurst, 389 p. Une traduction française a été publiée en 1997, sous le titre Rwanda 1959-1996: histoire d'un génocide, Milan, Dagorno, 514 p.

- PRUNIER G. (1997), «La crise au Kivu et ses conséquences dans la région des Grands Lacs», Géopolitique d'une Afrique médiane, Hérodote, 86-87, pp. 42-58.

- PRUNIER G. (2006), From genocide to continental war: the Congolese conflict and the crisis of contemporary Africa, London, Hurst, $450 \mathrm{p}$.

- PYPE K. (2007), «Fighting Boys, Strong Men and Gorillas: Notes on the Imagination of Masculinities in Kinshasa», Africa, 77, 2, pp. 250-271.

- QUAEGHEBEUR M. \& BIBIANE TSHIBOLA (dir.) (2007 et 2008), Aspects de la culture à l'époque coloniale en Afrique centrale, Congo-Meuse, 6-7, 2007, 8-9, 2008, Paris, L'Harmattan.

- RAEYMAEKERS T., voir VLASSENROOT K. (2008).

- RAHMANI M. (2002), Shalom Bwana. La saga des Juifs du Congo, Paris, Romillat. - RAPOPORT H. (1993), "L'approvisionnement vivrier de Kinshasa (Zaïre). Stratégies d'adaptation à la crise du sys- 
tème alimentaire", Paris, L'Harmattan, Cahiers des Sciences Humaines, 29, 4, ORSTOM, pp. 695-711.

- REMY G. (1993), «Image géographique de l'infection à $\mathrm{VIH} 1$ en Afrique centrale: des discontinuités remarquables", Annales de la Société belge de Médecine tropicale, 73, pp. 121-142.

- RENTON D., SEDDON D., ZEILIG L. (2007), The Congo. Plunder and Resistance, London, New York, Zed Books, $243 \mathrm{p}$.

- REYNTJENS F. (1993), «Démocratisation et conflits ethniques au Rwanda et au Burundi", Liber Amicorum Marcel d'Hertefelt, Cahiers Africains, 4-5, pp. 209-227.

- REYNTJENS F. (1994), L'Afrique des Grands Lacs en crise: Rwanda, Burundi: 1988-1994, Paris, Karthala, 326 p.

- REYNTJENS F. (1995), Rwanda. Trois jours qui ont fait basculer l'histoire, Cahiers Africains, 16, Bruxelles, Institut Africain-CEDAF, Paris, L'Harmattan, $151 \mathrm{p}$.

- REYNTJENS F. (1997a), «La rébellion au Congo-Zaïre: une affaire de voisins», Géopolitique d'une Afrique médiane, Hérodote, 86-87, pp. 55-77.

- REYNTJENS F. (1997b), «Rwanda et Burundi: les acteurs politiques», in VERHASSELT Y. (Actes édités par), RwandaBurundi. Journée d'étude, Bruxelles, ARSOM, pp. 111-126.

- REYNTJENS F. (1999), La guerre des Grands Lacs: alliances mouvantes et conflits extraterritoriaux en Afrique centrale, Paris, L'Harmattan, $255 \mathrm{p}$.

- REYNTJENS F. (2009), De grote Afrikaanse Oorlog. Congo in de regionale geopolitiek 1996-2006, Meulenhoff/Manteau, $364 \mathrm{p}$.

- REYNTJENS F., voir MARYSSE S.

- RIOT T. (2008), «Pratiques du corps, ethnicité et métissages culturels dans le Rwanda colonial (1945-1952)», Cahiers d'Etudes africaines, XLVIII, 4, $\mathrm{n}^{\circ}$ 192, pp. 915-833.

- RISHIRUMUHIRWA T. (1994), «Facteurs anthropiques de l'érosion dans les montagnes et hauts plateaux au Burundi, Rwanda et Zaïre», Les Cahiers d'OutreMer, Bordeaux, pp. 23-24.

- ROCHE E. (1996), «L'influence anthro- pique sur l'environnement à l'Age du Fer ancien dans le Rwanda actuel», GEOECO-TROP, 20, 1-4, pp. 73-89.

- ROCHE E. (1998), «Evolution du paléoenvironnement holocène au Rwanda. Implications climatiques déduites de l'analyse palynologique de séquences sédimentaires", in DEMAREE G. et al., International Conference on Tropical Climatology, Meteorolgy and Hydrology, In Memoriam Franz Bultot, Brussels, May 1996, ARSOM, pp. 108127

- ROCHE E. \& NTAGANDA Ch. (1998), «Analyse palynologique de la séquence KIHUNGU II (Région des Birunga, Rwanda). Evolution du paléoenvironnement et du paléoclimat dans le domaine afro-montagnard du Rwanda au cours de l'Holocène», GEO-ECO-TROP, Numéro spécial, 22, Actes du 4e Symposium de Palynologie Africaine, Sousse, Tunisie, pp. 71-82.

- ROCHE E., voir HABIYAREMYE F.X. (2003), MOSCOL-OLIVEIRA (1997).

- ROMAIN V. (2001), «Un espace culturel colonial: musique africaine en Belgique et au Congo", in VELLUT J.-L. (dir.), Itinéraires croisés de la modernité. Congo belge (1920-1950), Cahiers Africains, 4344, pp. 205-238.

- ROMANIUK A. (2006), Démographie congolaise au milieu du XXe siècle. Analyse de l'enquête sociodémographique par sondage des années 1955-1957. République démocratique du Congo, UCL, Presses Universitaires de Louvain, $217 \mathrm{p}$.

- RÖSLER M. (2004), «Regenwaldkolonisatie als Frontier-Prozess. Der Ituri-Wald in Nordost-Kongo (Zaïre) 1885-1995», Afrika Spectrum, 39, 3, Hamburg, pp. 335-357.

- ROYER A. (2004), «L'instrumentalisation politique des réfugiés du Kivu entre 1994 et 1996", in GUICHAOUA A. (dir.), Exilés réfugiés, déplacés en Afrique centrale et orientale, Paris, Karthala, pp. 425-528.

- RUBBERS B. (2003), Devenir médecin en République démocratique du Congo. La trajectoire socioprofessionnelle des diplômés en médecine de l'Université de Lubumbashi, Cahiers africains, 56, Tervuren, Institut Africain-CEDAF, Paris, 
L'Harmattan, $130 \mathrm{p}$.

- RUBBERS B. (2007), "Retour sur le "secteur informel". L'économie du Katanga (Congo-Zaïre) face à la falsification de la loi», Sociologie du Travail, 48, 3, pp. 316-329.

- RUBBERS B., voir KAHOLA TABU O. (2008).

- RUNGE J. (1996), «Paleaoenvironmental interpretations of geomorphological and pedological studies in the rain forest "core-areas" of Eastern Zaire (Central Africa)", South African Geographical Journal, 78, 2, pp. 91-97.

- RUNGE J. (2001), «ERS-1 SAR dataapplied geological and geomorphological mapping for structural and civil engineering in rain forest areas of the eastern D.R.Congo (Kivu), Central Africa», Zeitschrift für Geomorphologie, Supplementband, $n^{\circ} 124$, pp. 71-87.

- RUNGE J. (2001), «Landschaftsgenese und Paläoklima in Zentralafrika. Physiogeographische Untersuchungen zur klimagesteuerten quartären Vegetations-und Geomorphodynamik in Kongo-Zaire (Kivu, Kasai, Oberkongo) und der Zentralafrikanischen Republik (Mbomou)", Relief-Boden-Paläoklima, 17, pp. 1-294.

- RUNGE J. (2002), «Wie alt is der Regenwald? Umweltgeschichtliche Forschungen in Kongobecken Zentralafrikas», Forschung Frankfurt, 1-2, pp. 2229

- RUNGE J. (2000), voir MERCADER J.

- RURANGWA E., voir VERHEUST L. (1995).

- RYCKMANS G. (présenté par) (1995), André Ryckmans. Un territorial au Congo belge. Lettres et documents 1954-1960, Coll. Zaïre. Histoire et Société, Centre de I'histoire de l'Afrique UCL, Paris, L'Harmattan, $426 \mathrm{p}$.

- SABAKINU KIVILU (dir.) (1999a), Démocratie et paix en République démocratique du Congo, Presses de l'Université de Kinshasa, $270 \mathrm{p}$.

- SABAKINU KIVILU (1999b), «La Conférence nationale souveraine comme enjeu de la démocratie et de la paix", in SABAKINU KIVILU (dir.), Démocratie et paix en République démocratique du Congo, Presses de l'Université de Kinshasa, pp. 47-56.
- SABAKINU KIVILU I. (dir.) (2000), Elites et Démocratie en République démocratique du Congo, Kinshasa, Presses de I'Université de Kinshasa.

- SABAKINU KIVILU I. (2001), «A la recherche du paradis terrestre: les Bana Luunda entre le diamant et le dollar», in MONNIER L., JEWSIEWICKI B. \& DE VILLERS G. (2001), Chasse au diamant au Congo/Zaïre, Cahiers Africains, 45-46, Tervuren, Institut Africain-CEDAF, Paris, L'Harmattan, pp. 127-169.

- SABAKINU KIVILU (2004), «Les violences au quotidien dans la ville de Kinshasa sous Mobutu (1965-1997)», in MABIALA MANTUBA NGOMA P. (dir.), La Nouvelle Histoire du Congo. Cahiers Africains, 65-66-67, pp. 289-308.

- SABAKINU KIVILU (2004), «Les populations réfugiées dans l'Ouest de la République démocratique du Congo", in GUICHAOUA A. (dir.), Exilés réfugiés, déplacés en Afrique centrale et orientale, Paris, Karthala, pp. 567-629.

- SALAUN J., voir KARCH S. (1993).

- SALMON P. (1992), «Un prélude à janvier 1959?», Congo 1955-1960, Recueil d'études, Bruxelles, ARSOM, pp. 359370.

- SALMON P. (1995a), «La poursuite du trafic négrier sur la côte occidentale de l'Afrique (juillet-août 1859)», Bull. ARSOM, pp. 323-343.

- SALMON P. (1995b), "Les retours en Belgique induits par la décolonisation», in MIËGE J.-L. \& DUBOIS C. (dir.), L'Europe retrouvée. Les migrations de la décolonisation, Paris, L'Harmattan, pp. 191-212.

- SALMON P. (1995c), «Le voyage au Congo du lieutenant-général Godfroid», R.B.G., pp.175-188.

- SALMON P. (1998), «De l'ethnie zande à la nation congolaise. Un parcours tourmenté vers la démocratie», Bull. ARSOM, Bruxelles, 44, 4, pp. 567-581.

- SALMON P. (2004), "Lettres et carnets de route des pionniers belges. Etude des mentalités africaines», Bull. ARSOM, 50, 2, pp. 167-182.

- SALMON P. (2007), Nouvelle introduction à l'histoire de l'Afrique, Paris, L'Harmattan, Bruxelles, Institut de Sociologie, $299 \mathrm{p}$.

- SALOMON J.-N (1998), «Les phéno- 
mènes d'érosion accélérée du plateau du Kwango (République Démocratique du Congo)", Travaux du Laboratoire de Géographie Physique Appliquée, Institut de Géographie, Université Michel de Montaigne-Bordeaux 3, n¹6, 1997-1998, pp. 45-63.

- SAMBA-KIMBATA M.J. (1995), «La déforestation dans la région du Pool (Congo)», GEO-ECO-TROP, 19, 1-4, pp. 105-118.

- SAMBA G., MALOBA MAKANGA J.D., MBATI R. (1999- 2000), "Les saisons des pluies potentiellement utiles (S.P.P.U.) sur les plateaux Batéké et sur le plateau des Cataractes en République du Congo", Publications de l'Association internationale de climatologie, 12, pp. 190-197.

- SANGA-NGOIE K. \& FUKUYAMA K. (1996), «Interannual and long-term climate variability over the Zaire River Basin during the last 30 years», Journal of Geophysical Research, 101, pp. 2135121360.

- SCHOENBRUN D. L. (1994-1995), «Social aspects of agricultural change between the Great Lakes: AD 500 to 1000», Azania, XXIX-XXX, pp. 270-282.

- SCHOONHEYT J. (2005), «La collection de moyens d'échange du Cabinet des Médailles de Bruxelles: un ensemble d'objets importés en Afrique centrale pour l'administration de l'Etat Indépendant du Congo», Bull. ARSOM, 51, 4, pp. 465-486.

- SCHWARTZ D. (1996), «Archéologie préhistorique et processus de formation des stone-lines en Afrique centrale (Congo-Brazzaville et zones périphériques)», GEO-ECO-TROP, 20, 1-4, pp. 1538.

- SCHWARTZ D., voir BONNEFILLE P. (2002).

- SEBAKUNZI NTIBIBUKA, BIREMBANO BAHATI (1993), «Tentatives d'aménagement des marais du Bushi», L'eau et l'aménagement dans l'Afrique des Grands Lacs, Colloque Bujumbura, 1991, Talence, CRET, pp. 129-144.

- SEDDON D., voir RENTON D. (2007).

- SERUFURI KAKIZA P. (2004), «Les Etats-Unis d'Amérique et l'enseignement en Afrique noire (1910-1948)», in MABIALA MANTUBA-NGOMA P. (dir.), La
Nouvelle Histoire du Congo, Cahiers Africains, 65-66-67, pp. 229-249.

- SHARP P. M. \& HAHN B. H. (2008), "Aids: Prehistory of HIV-1», Nature, 455, 7213, pp. 605-606.

- SIDA et développement en Afrique (1995), Publication à l'occasion de l'attribution du Prix International Roi Baudouin pour le Développement à The Aids Support Organization (TASO) (Bruxelles, le 22 mars 1995), Bulletin ARSOM, 41. Supplément $n^{\circ} 1,88 p$.

- SIDDIQUE A.K., SALAM A. et al. (1995), "Why treatment centres failed to treat cholera deaths among Rwandan refugees in Goma, Zaire», The Lancet, 345, pp. 359-361.

- SIMONS E. (1993), Inventaire des études africaines en Belgique. Inventaris van de Afrika studies in België, Cahiers Africains-CEDAF, Bruxelles, L'Harmattan, Paris, $n^{\circ} 1-2,341 \mathrm{p}$.

- sibamana C. et MOEYERSONS J. (1996), "La problématique de la lutte antiérosive au Rwanda», Bull. SOBEG, LXV, 1, pp. 49-74.

- SIMA J. (2001), «Les Bana-Lunda et les transformations socioéconomiques à Kikwit», Congo-Afrique, 355, pp. 297-310. - SIMONARD T., DUCHAUFOUR H., BIZIMANZ M. \& MIKOKORO C. (1994), «La conservation des sols en milieu paysan burundais. Etude et hiérarchisation des stratégies anti-érosives», Bordeaux, Les Cahiers d'Outre-Mer, 185, pp. 48-64.

- SIMONS E., voir VERHAEGEN (2006).

- SIRVEN P. (éd.) (1993), L'eau et l'aménagement dans l'Afrique des Grands Lacs, Coll. Pays enclavés, Colloque Bujumbura 1991, Talence, CRET, n5, 372 p.

- SMITH R. (2003), «"Here we are in Full Battle": Jesuits and Baptists in the Kwilu, 1912-1940», American Baptist Quarterly, XXII, 2, pp.180-211.

- SMITH R.E. (2005), «Les Kwilois parlent de l'époque coloniale», Annales Aequatoria, 26, pp.165-217.

- SOLOTSHI M., voir ASSANI A.A. (1993), KAYEMBE C.M.P. (1993), ASSUMANI (1993).

- STACHE N., DRECHSEL P. (1997), «Ökologischer Landbau in der ländlichen Entwicklung Ruandas», Geographische 
Rundschau, 49, 1, pp. 32-38.

- STACHE N., WIRTHMANN A. (1998), «Die Misere in der Landwirtschaft im tropischen Afrika am Beispiel einer geoökologischen Studie in Ruanda», Petermanns geographische Mitteilungen, 142, 5-6, pp. 339-354.

- STAMM A.L., voir WILLIAM D.B. (1995). - STENGERS J. (1993), "Sur la critique des données démographiques en Afrique», in THOVERON G. \& LEGROS H., (éd.), Mélanges Pierre Salmon, II, pp. 377-401, Civilisations, XLI, 1, pp. 25-409. - STENGERS J. (1995), «La décolonisation du Congo: un modèle belge», in AGERON C.R. \& MICHEL M. (dir.), L'ère des décolonisations, sélection de textes du colloque "Décolonisations comparées", Aix-en-Provence, 30 septembre3 octobre 1993, pp. 307-317.

- STENMANS A. \& REYNTJENS F. (1993), La pensée politique du gouverneur général Pétillon, ARSOM, Classe des sciences morales et politiques, Mém. in- $8^{\circ}$, nelle série, vol. 53, fasc. 3, $123+17 \mathrm{p}$.

- STROTHER Z. (2004), «Architecture against the State. The Virtues of Impermanence in the "kibulu" of the Eastern Pende Chief in Central Africa», Journal of the Society of architectural Historians, 63, 3, September, pp. 272295.

- STROTHER Z. (1998), Inventing Masks. Agency and History in the Art of the Central Pende, Chicago, London, The University of Chicago Press, XXVII + 348 p.

- STROTHER Z. (2005), «La perte et l'efflorescence. Paradoxes de l'histoire de l'art colonial dans le Pende central», in VELLUT J.-L. (dir.), La mémoire du Congo. Le temps colonial, AfricaTervuren, Editions Snoeck, pp. 115-119.

- StROTHER Z. (2008), Pende, Coll. Visions d'Afrique, Milan, 5 Continents Editions.

- STURTEWAGEN C. (2006), «Un Royaume hamite van P. Pagès (Brussel 1933). Een egyptologische terugblik», Bull. ARSOM-KAOW, 52, 2, pp. 101-112.

- SYMOENS J.-J. (2001), «La botanique et la zoologie», in HALLEUX R. et al. (dir. scientifique), Histoire des sciences en Belgique 1815-2000, vol. 2, La Renais- sance du Livre, Dexia, pp. 255-266.

- SYS C. \& VAN RANST E. (1996), «Population Supporting Capacity of the Kivu Landscape (Zaire) under Traditional and Intensive Farming", Bull. ARSOM, 42, 2, pp. 242-272.

- SWENNEN R., voir DE LANGHE E. (1994-1995).

- TACK L., FERNANDEZ-ALONSO M., CHARTRY G., ERRERA M., LAVREAU J., TAHON A. \& FRANCESCHI G. (2006), "The Great Lakes'Region of Central Eastern Africa: Overrated Mineral and Hydrocarbon Potential in the Western Rift», in DE DAPPER M. \& de LAME D. (eds.), Africa's Great Rift: Diversity and Unity, Africa-Tervuren, ARSOM, pp. 320321

- TAELMAN H., voir DELACOLLECTE C. (1995).

- TAHON A., voir TACK L. (2006).

- TEGERA A. (1995), «La réconciliation communautaire: le cas des massacres au Nord-Kivu», in GUICHAOUA A. (dir.), Les crises politiques au Burundi et au Rwanda (1993-1994). Analyses, faits et documents, Villeneuve d'Ascq, Université des Sciences et Technologies de Lille, pp. 395-402.

- TEHEBWA MUNDA (2008), «La rumba entre traite négrière et ère coloniale belge", in QUAEGHEBEUR $\mathrm{M}$. et $\mathrm{BI}$ BIANE TSHIBOLA (dir.), Aspects de la culture à l'époque coloniale en Afrique centrale, Congo-Meuse, vol. 9, Paris, L'Harmattan, pp.203-226.

- TELLIER V., LUBOYA N., DE GRAEVE G., BEGHIN Y. (1996), "Le rôle des familles dans la réhabilitation nutritionnelle ambulatoire», Symposium «Enfance dans le Tiers Monde», Bruxelles, 20-22 octobre 1994. Actes publiés sous la dir. de J.-J. SYMOENS, Bruxelles, ARSOM, pp. 125-137.

- TEMMERMAN M, voir MICHIELSEN K. (2008).

- THIBON Ch. (2004), Histoire démographique du Burundi, Paris, Karthala $483 \mathrm{p}$.

- THIBON C., voir GAHAMA J. (1994).

- THIRY E. (1996), Une introduction à l'ethnohistoire des Hema du Sud (HautZaïre), Tervuren, MRAC, Annales, Sciences humaines, 150, $321 \mathrm{p}$. 
- THYS VAN DEN AUDENAERDE D. (2002), "Over historisch, sociohistorisch en ethnohistorisch onderzoek te Tervuren», FORUM, ABA-BVA, 23, pp. 9-13.

- TILMAN S. (2001), “L'implantation du scoutisme au Congo belge», in VELLUT J.-L. (dir.), Itinéraires croisés de la modernité. Congo belge (1920-1950), Cahiers Africains, 43-44, pp. 103-140.

- TOLLENS E. (1994), «Een voorbeeld van informele economie: de voedselbevoorrading van Kinshasa», Bull. ARSOM, 40, Suppl. n¹, pp. 27-34.

- TOLLENS E. (2003), "L'état actuel de la sécurité alimentaire au Congo. Diagnostic et perspectives», Bull. ARSOM, 49, 4, pp. 471-494

- TOLLENS E. (2004), «Sécurité alimentaire à Kinshasa: un face-à-face quotidien avec l'adversité», in TREFON Th. (dir.), Ordre et désordre à Kinshasa, Cahiers Africains, 61-62, Africa Tervuren, Paris, L'Harmattan, pp. 61-79.

- TOLLENS E., voir GOOSSENS F. (1994), MUKADI KANKONDE (2001).

- TOMBU C., voir HUART A. (2009).

- TONDA J. (2008), «La violence de l'imaginaire des enfants-sorciers", Cahiers d'Etudes africaines, XLVIII, 1-2, n¹89-190, pp. 325-343.

- TONGLET R., voir GOYENS Ph. (1996).

- TOWLES J. A. (1993), NKUMBI Initiation. Rituals and Structure among the Mbo of Zaïre, Tervuren, MRCA, Annales, Sciences humaines, vol. 137, $180 \mathrm{p}$.

- TREFON Th. (2000), «Population et pauvreté à Kinshasa», Afrique contemporaine, 194, Paris, $2^{\mathrm{e}}$ trim., pp. $82-80$.

- TREFON Th. (2002), «The Political Economy of Sacrifice: Kinois and the State», Review of African Political Economy, 93-94, pp. 481-498.

- TREFON Th. (dir.) (2004), Ordre et désordre à Kinshasa. Réponses populaires à la faillite de l'Etat, Cahiers Africains, 61-62, Tervuren, MRAC, Paris, L'Harmattan, $252 \mathrm{p}$.

- TREFON Th. (ed.) (2004), Reinventing Order in the Congo. How People respond to State Failure in Kinshasa, London, Zed Books, 224 p. Version anglaise de l'ouvrage précédent.

- TREFON Th. (avec la coll. de NGOY B.) (2007), Parcours administratifs dans un
Etat en faillite. Récits populaires de Lubumbashi (RDC), Cahiers Africains, 74, Africa-Tervuren, Paris, L'Harmattan, $154 \mathrm{p}$.

- TREFON Th. \& COGELS S. (2005), «A Stakeholder Approach to Natural Resources Management in Peri-urban Central Africa», in DE DAPPER M. (ed.), International Symposium. Tropical Forests in a Changing Global Context, ARSOM, pp. 197-221.

- TROUWBORST A. (1993), «Rwanda en Burundi. Twee ethnografische tradities?», Liber Amicorum Marcel d'Hertefelt, Essais anthropologiques (sous la dir. de P. Wymeersch), Cahiers Africains, Afrika Studies, 4-5, Bruxelles, Institut AfricainCEDAF, pp. 255-270.

- TSHIALA L. (2000), «Des hommes et des vaches. Le phénomène mutumbula au Congo", in MONNIER L. \& DROZ I. (dir.), Côté jardin, côté cour: l'anthropologie de la maison africaine, Paris, P.U.F., pp. 141-179.

- TSHIBANGU KABET MUSAS (1995), «La solution de la grande crise mondiale des années 1930 au Congo», ZaïreAfrique, 291, pp. 39-55.

- TSHIBANGU K.W.T., ENGELS P. \& MALAISSE F. (1997), «Evolution du couvert végétal dans la région de Kinshasa (1960-1987) selon une approche cartographique», GEO-ECO-TROP, 21, 1-4, pp. 95-103.

- TSHIBANGU K. \& MALAISSE F. (1995), «L'approvisionnement en bois de feu de Kinshasa (Zaïre). Note préliminaire», GEO-ECO-TROP, 19, 1-4, pp. 119-129.

- TSHILEMALEMA MUKENGE (2002), Culture and Customs of the Congo, Collection Culture and Customs in Africa, Westport, Connecticut, London, Greenwood Press, $X X+204$ p.

- TSHIMANGA C. (1999), "Scoutisme et formation de l'élite au Congo-Kinshasa», Congo-Afrique, 333.

- TSHIMANGA C. (2001a), «L'ADAPES et la formation d'une élite au Congo (19251945)», in VELLUT J.-L. (dir.), Itinéraires croisés de la modernité. Congo belge (1920-1950), Cahiers Africains, 43-44, pp. 189-204.

- TSHIMANGA C. (2001b), Jeunesse, formation et société au Congo/Kinshasa, 
1890-1960, Paris, L'Harmattan, 444 p. - TSHIMANGA C., voir VERHAEGEN B. (2003).

- TSHIMANGA Cl. (2004.), «Analyse multidimensionnelle de la pauvreté dans les ménages de Kisenso", Vivre et survivre à Kinshasa. Problématique du développement humain, pp. 181-210.

- TSHIMANGA MBUYI Cl., voir LELO NZUZI F (2004)

- TSHINGOMBE MULUBAY F. (2008), «La RDC face à la crise alimentaire mondiale», Congo-Afrique, 429, pp. 736-746. - TSHUNGU BAMESA (dir.) (2000), Finances et démocratie en République démocratique du Congo. L'impôt sans le pot, Kinshasa, Publications de I'Institut pour la Démocratie et le Leadership politique, $186 \mathrm{p}$.

- TULET J.-Ch., CHARLERY B., BART F. \& PILLEBOUE J. (dir.) (1994), Paysanneries du café des hautes terres tropicales. Afrique et Amérique latine, Paris, Karthala.

- TURNER T. (2000), Ethnogenèse et nationalisme en Afrique centrale. Aux racines de Patrice Lumumba, Paris, L'Harmattan, $456 \mathrm{p}$.

- TWAIN M. (2004), Le Soliloque du roi Léopold, Paris, L'Harmattan.

- UWIZEYIMANA L. (1996), «Crise du café, faillite de l'Etat et implosion sociale au Rwanda», GEODOC, 52, Série MOCA, $n^{\circ} 4$, Montagnes et Café, $134 \mathrm{p}$.

- UWIZEYIMANA L. (2001), La caféiculture au Rwanda: stratégies paysannes et logiques étatiques, Table Ronde, 27-111998, Collection Espaces tropicaux, Pessac, DYSMET, $n^{\circ} 16$, pp. 579-594.

- VALLÉE O., voir MISSER F. (2001).

- VAN ACKER F. et VLASSENROOT K. (2001), "Les "Maï-Maï" et les frontières de la violence milicienne dans l'est du Congo", Politique africaine, 84, pp. 103116.

- VAN ACKER F., voir VLASSENROOT K. (2001).

- VAN BALEN H. (1997), «Relaas en achtergrond van het Kasongo-project», Bull. ARSOM, 43, 2, pp. 157-179.

- VAN BORTEL W., BARUTWANAYO M., DELACOLETTE C., COOSEMANS $M$. (1996), «Motivations à l'acquisition et à l'utilisation des moustiquaires imprégnées dans une zone à paludisme stable au Burundi», Tropical Medicine \& International Health, 1, 1, pp. 71-80.

- VAN CLEEMPUT O., voir BOECKX P. (2006).

- VANDEN EEKHOUT A., voir BRUNEEL H. (1994).

- VAN DEN HERREWEGEN M., DE DONCKER F., VOET P. \& VANDEN BERGHE I. (2006), «Revision of the Southern Part of the Geodetic Network of the Democratic Republic of Congo", Bull. ARSOM, 52, 2, pp. 337-350.

- VANDEN BERGHE I., voir VAN DEN HERREWEGEN M. (2006).

- VANDERLINDEN J. (1994), Pierre Ryckmans, 1891-1959: coloniser dans l'honneur, avec un libre propos de J. Stengers, Bruxelles, De Boeck-Wesmael, $\mathrm{XI}, 802 \mathrm{p}$

- VANDERLINDEN J. (2004), «Regards sur la rencontre d'un ordre colonial et d'ordres précoloniaux. Fragments relatifs au destin de droits originellement africains dans le système juridique colonial belge», in LAMY E. et DE CLERCK L. (éd.), L'ordre juridique colonial belge en Afrique centrale. Eléments d'histoire, Recueil d'études, Bruxelles, ARSOM pp. 359-438.

- VANDERLINDEN J. (2007), Main-d'oeuvre, Eglise, Capital et Administration dans le Congo des années trente (Vol.1), Fontes Historiae Africanae, ARSOM, Bruxelles, $331 \mathrm{p}$.

- VANDERSTRAETEN L.-F. (2001), La répression de la révolte des Pende du Kwango, ARSOM, Classe des Sciences morales et politiques, Mémoire in- $8^{\circ}$, Nouvelle Série, Tome 53, fasc.1, Bruxelles, $146 \mathrm{p}$.

- VANGROENWEGHE D. (2000), Sida et sexualité en Afrique, EPO, 479 p. L'édition originale, publiée en 1997, a comme titre: Aids in Africa. Oorsprong, verspreiding en seksuele netwerken, Antwerpen, EPO. - VANHEE H. (1996), «Territoriale culten in West-Congo: verdwijning of transformatie?», Afrika Focus, 14, 1, pp. 55-65.

- VANHEE H. en CASTRYCK G. (2002), «Belgische historiografie en verbeeldingen over het koloniale verleden", Revue Belge d'Histoire Contemporaine, XXXII, 34, pp. 305-320. 
- VANHEE H., voir ARNAUT K. (2001).

- VAN OVERLOOP E. \& PAEPE R. (1998), «The Quaternary Geology around the Great Lakes in Central Africa. Pluvials and Interpluvials are not the equivalent of Glacials and Interglacials», in DEMARÉE G. et al., International Conference on Tropical Climatology, Meteorology and Hydrology, In Memoriam Franz Bultot, Brussels, May 1996, ARSOM, pp. 142175.

- VAN PEEL B. (2001), «Aux débuts du football congolais», in VELLUT J.-L. (dir.), Itinéraires croisés de la modernité. Congo belge (1920-1950), Cahiers Africains, 4344, pp. 141-188.

- VAN RANST E., voir BAERT G. (1998), GALLEZ A. (1997), IMERZOUKENE S. (2001), SYS C. (1996), VERDOODT A. (2006).

- VAN SCHUYLENBERGH P. (1997), La mémoire des Belges en Afrique centrale. Inventaire des archives historiques privées du Musée royal de l'Afrique centrale de 1858 à nos jours (sous la dir. de Philippe Marechal), Tervuren, M.R.A.C., Inventaire des Archives historiques, vol. 8, $149 \mathrm{p}$.

- VANSINA J. (1993a), “Comment l'histoire se construit: la conquête du royaume kuba (1899-1900)», Mélanges Pierre Salmon, I, Civilisations, XI, 2, pp. 41-49.

- VANSINA J. (1993b), «Zairian Masks in Historical Perspective", in HERREMAN F., PETRIDIS C. (eds.), Face of the Spirits. Masks from the Zaire Basin, Tervuren, MRAC, Annales Sciences humaines, 140, pp. 235-247.

- VANSINA J. (1993c), "Nieuws over de "oorsprong" der Tuutsi", Liber Amicorum Marcel d'Hertefelt, Cahiers Africains, 4-5, - VANTIEGHEM L. (1994), «La culture du cacao au Mayombe (Congo belge) 18851914. Les bases d'une économie de plantation capitaliste", in Chocolat, de la boisson élitaire au bâton populaire, XVIe-XXe siècles, exposition CGER, Bruxelles, pp. 143-160.

- VAN WOLPUTTE S., DEVISCH R., LE ROY J. \& LAPIKA D. (2002), Medical pluralism and lay therapy management in Kinshasa, Uppsala-Leuven Research in Cultural Anthropology ULRICA 2, Uppsala, Department of Cultural Anthropology and Ethnology, $132 \mathrm{p}$.

- VELLUT J.-L. (1994a), «Ressources scientifiques, culturelles et humaines de l'africanisme en Belgique. Perspectives sur un patrimoine d'outre-mer et sa mise en valeur», in DE VILLERS G. (dir.), Belgique-Zaïre. Une histoire en quête d'avenir, pp. 115-144.

- VELLUT J.-L. (1994b), «La Force Publique dans l'histoire du Congo belge. Le "point de vue africain": quelques réflexions", Défense et maintien de l'ordre en Afrique Centrale à l'époque contemporaine de la colonisation belge (19081962), Actes du colloque international du 17 octobre 1992, Bruxelles, Musée royal de l'Armée, Centre d'Histoire militaire, Travaux, 28, pp. 63-74.

- VELLUT J.-L. (1996a), «En quête d'interprétation des crises de la modernité. L'historiographie immédiate de la prédication de Simon Kimbangu (1921)», in DE VILLERS G. (dir.), Phénomènes informe/s et dynamiques culturelles en Afrique, Cahiers Africains, 19-20, pp. 138-165.

- VELLUT J.-L. (1996b), «Le cacao dans l'économie politique de l'ancien Congo belge», Chocolat, de la boisson élitaire au bâton populaire, $X V I e_{-} X X^{e}$ siècles, exposition CGER, Bruxelles, pp. 121-142.

- VELLUT J.-L. (1999), «Prestige et pauvreté de l'histoire nationale. A propos d'une histoire générale du Congo", Revue belge de Philologie et d'Histoire, 77, pp. 480-517.

- VELLUT J.-L. (2000), «La Belgique et la préparation de l'indépendance du Congo", in LANOTTE O., ROOSENS C., CLÉMENT C. (dir.), La Belgique et l'Afrique centrale de 1960 à nos jours, GRIP, Bruxelles, Ed. Complexe, pp. 8394.

- VELLUT J.-L. (dir.) (2001a), Itinéraires croisés de la modernité. Congo belge (1920-1950), Cahiers Africains, 43-44, Tervuren, Institut-Africain-CEDAF, Paris, L'Harmattan, 295 p.

- VELLUT J.-L. (2001b), «Jan Vansina, alias Bope Muudy», BVA-ABA Forum, 21, pp. 3-6.

- VELLUT J.-L. (2002), «Jan Vansina on the Belgian historiography of Africa: Around the agenda of a bombing raid", BVA-ABA FORUM, 22, pp. 17-22. 
- VELLUT J.-L. (2004), «Réflexions sur la question de la violence dans l'histoire de l'Etat Indépendant du Congo", in MABIALA MANTUBA NGOMA P. (dir.), La Nouvelle Histoire du Congo. Cahiers Africains, 65-66-67, pp. 269-287.

- VELLUT J.-L. (dir.) (avec CORNÉLIS S., DE LAME D., DE VILLERS G., ZANA ETAMBALA, LAGAE J., MARECHAL P.) (2005a), La mémoire du Congo. Le temps colonial, Africa Tervuren, MRCA et Editions Snoeck, $271 \mathrm{p}$

- VELLUT J.-L. (2005b), Simon Kimbangu. 1921: de la prédication à la déportation. Les Sources, Vol. I. Fonds missionnaires protestants (1) Alliance missionnaire suédoise (Svenska Missionsförbundet SMF), ARSOM, Coll. Fontes Historiae Africanae, XXXII + 178 p. - VELLUT J.-L. (2006), «Angola-Congo. L'invention de la frontière du Lunda (18891893), Africana Studia, Revista Internacional de Estudos Africanos, $n^{\circ}$ 9, Edição do Centro de Estudos Africanos da Universidade do Porto (CEAUP), pp.159184, avec 5 cartes en couleurs h.-t.

- VELLUT J.-L. (dir.) (2007), Villes d'Afrique. Explorations en histoire urbaine, Cahiers Africains, 73, AfricaTervuren, FNRS, Chaire d'Etudes africaines UCL, Paris, L'Harmattan, $287 \mathrm{p}$. - VELLUT J.-L., voir LORIAUX F. (1996), WILUNGULA B. COSMA (1997).

- VERDIER R., DECAUX E., CHRETIEN J.P. (éd.) (1995), Rwanda, un génocide du $X X e$ siècle, Paris, L'Harmattan, $263 p$

- VERDOODT A., VAN RANST E. (2006), «The soil information system of Rwanda: a useful tool to identify guidelines towards sustainable land management», Afrika Focus, 19, 1-2, pp. 69-92.

- VERHAEGEN B. (1993), «Contribution à la biographie politique de Patrice Lumumba», Bull. ARSOM, 39, 4, pp. 597610.

- VERHAEGEN B. (1995), «Commentaires sur la biographie de Pierre Ryckmans (1891-1959) par Jacques Vanderlinden», Bull. ARSOM, 41, 2, pp. 173-181.

- VERHAEGEN B. (2000), “Guevara au Congo, 23 avril-22 novembre 1965», Bull. ARSOM, pp. 101-111.

- VERHAEGEN B. (avec la coll. de TSHIMANGA C.) (2003), L'ABAKO et l'indépendance du Congo belge. Dix ans de nationalisme kongo (1950-1960), Cahiers africains, 53-54-55, Tervuren, Institut Africain-CEDAF, Paris, L'Harmattan, $454 \mathrm{p}$

- VERHAEGEN B. (avec la coll. de OMASOMBO J., SIMONS E. \& VERHAEGEN B.) (2006), Mulele et la révolution populaire au Kwilu (République démocratique du Congo), Cahiers africains, 72, Africa Tervuren, Paris, L'Harmattan, $365 \mathrm{p}$

- VERHAEGEN B., voir OMASOMBO TSHONDA J (1998 et 2005).

- VERHAEGEN E. et DEGAND J. (1993), L'évolution des systèmes agricoles au Burundi. Une dynamique conflictuelle, Cahiers CIDEP, 17, Louvain-la-Neuve, Academia, $100 \mathrm{p}$

- VERHASSELT Y. (Actes édités par) (1997), Rwanda-Burundi. Journée d'étude, Bruxelles, le 10 janvier 1997, ARSOM, 173 p. - VERHEUST L., RURANGWA E. et OLLEVIER F. (1995), «La polyculture TilapiaClarias au Rwanda, avec attention particulière à l'intégration pisciculture-agriculture-élevage», Séminaire "L'aménagement des écosystèmes agro-piscicoles d'eau douce en milieu tropical'» (Bruxelles, 16-19 mai 1994), Actes publiés sous la dir. de J.-J. Symoens et J.-C. Micha, Bruxelles, CTA (Centre technique de coopération agricole et rurale), pp. 435-446.

- VERSCHUREN J. (1993), «Les habitats et la grande faune: évolution et situation récente», Exploration du Parc National des Virunga (Zaïre), 2e série, fasc.29, Fondation pour favoriser les recherches scientifiques en Afrique, Bruxelles, $133 \mathrm{p}$. - VIDAL Cl. (1993), «Marcel d'Hertefelt et l'énigme des clans rwandais. Un style de recherches", Liber Amicorum Marcel d'Hertefelt, Cahiers Africains, Afrika Studies, 4-5, pp. 325-330.

- VIDAL Cl. (1998), «Questions sur le rôle des paysans pendant le génocide des Rwandais", Cahiers d'Etudes africaines, 150-152, pp. 331-345.

- VIDAL Cl. (2004), «La commémoration du génocide au Rwanda. Violence symbolique, mémorisation forcée et histoire officielle», Cahiers d'Etudes africaines, 175, pp. 575-592.

- VINK H. (2000a), «Etat de la recherche sur les bonobos de l'Equateur (R.D. Congo)», 
Annales Aequatoria, 21, pp. 33-40.

- VINK H. (2000b), «De studie van het schoolboek in Belgisch Kongo", Bulletin ARSOM, 46, 2, pp. 83-100.

- VIS H. (1997), «De voeding en de nutritionele toestand van de bevolking in het gebied van de Grote Meren van CentraalAfrika", in VERHASSELT Y. (éd.), Rwanda-Burundi, Journée d'études, Bruxelles, ARSOM, pp. 63-80.

- VIS H., GOYENS P. \& BRASSEUR D. (1994), «Rwanda: the case for research in developing countries», The Lancet, 344, 8927, pp. 957.

- VIS H. \& GOYENS P. (1996), «La coopération au développement et les aides humanitaires (l'exemple de l'Afrique centrale des Grands Lacs)", Séances académiques sur le thème "Problèmes de la Coopération au Développement», Paris, 13 et 14 mai 1995, Paris, Académie des Sciences d'Outre-Mer, Bruxelles, ARSOM, pp. 21-48.

- VIS H., voir BRASSEUR D. (1998).

- Vivre et survivre à Kinshasa. Problématique du développement humain (2004), Observatoire d'Economie politique et de Développement humain, Université d'Anvers, Facultés catholiques de Kinshasa, Afrique et développement 19, Etudes publiées par la Faculté d'Economie et Développement et Notes de conjoncture, numéro spécial, $321 \mathrm{p}$.

- VLASSENROOT K. (2002), «Violence et constitution de milices dans l'est du Congo: le cas des Mayi Mayi», in REYNTJENS F. \& MARYSSE S., L'Afrique des Grands Lacs, Annuaire 2001-2002, pp. 115-152.

- VLASSENROOT K. (2004), «Land and Conflict. The Case of Masisi», in VLASSENROOT K. \& RAEYMAEKERS T. (eds.), Conflict and Social Transformation in Eastern DR Congo, pp. 81-101.

- VLASSENROOT K. \& RAEYMAEKERS T. (eds.) (2004), Conflict and Social Transformation in Eastern DR Congo, Ghent, Academia Press.

- VLASSENROOT K., RAEYMAEKERS T. (2008), «New political order in the DR Congo? The transformation of regulation», Afrika Focus, 21, 2, pp. 39-52.

- VLASSENROOT K., VANACKER F. (2001), «War as exit for exclusion? The formation of Mayi-Mayi militias in Eastern Congo", Afrika Focus, 17, 1-2, pp. 51-77. - VOET P., voir VAN DEN HERREWEGEN M. (2006).

- VRYDAGHS L. (2000), voir MERCADER J.

- VUYLSTEKE D. voir DE LANGHE E. (1994-1995)

- WAETERLOOS E., voir MARYSSE S. (1993).

- WAFUlA M., voir MUNYOLOLO Y. (1994).

- WARRIER R.J., voir KUVUBIDILA S. (1993).

- WEISS H.E., Radicalisme rural et lutte pour l'indépendance au Congo-Zaïre. Le parti solidaire africain (1959-1960) (avec une préface d'Immanuel Wallerstein), Paris, L'Harmattan, $350 \mathrm{p}$.

- WÉRY M., voir DELACOLLECTE C. (1995).

- WIESE B. (1997), «Flüchtlinge zwischen Ruanda und Zaire. Hintergründe und Perspektiven des Konflikts an der Grossen Seen», Ökologie der Tropen, Geographische Rundschau, 49, 1, pp. 54-56.

- WILLAME J.-C. (1995), Aux sources de I'hécatombe rwandaise, Cahiers Africains, 14, Bruxelles, Institut AfricainCEDAF, Paris, L'Harmattan, $175 \mathrm{p}$.

- WILLAME J.-C. (1996), L'ONU au Rwanda, 1993-1995, La Communauté internationale à l'épreuve d'un génocide, Bruxelles, Editions Labor-Editions, Paris, Maison-Neuve Larose, 175 p.

- WILLAME J.-C. (1997a), «Gestion verticale et horizontale des crises identitaires: le cas du Kivu montagneux», Géopolitique d'une Afrique médiane, Hérodote, 86-87, pp. 78-115.

- WILLAME J-.C. (1997b), Banyarwanda et Banyamulenge, Violences ethniques et gestion de l'identitaire au Kivu, Cahiers Africains, Institut Africain-CEDAF, Tervuren, L'Harmattan, Paris, $n^{\circ} 25,156 \mathrm{p}$.

- WILLAME J.-C. (1997c), Les Belges au Rwanda: le parcours de la honte, GRIP, Bruxelles, Editions Complexe, 207 p.

- WILLAME J.-C (1999), L'Odyssée Kabila: trajectoire pour un Congo nouveau, Paris, Karthala, 250 p.

- WILLAME J.-Cl. (2002), L'accord de Lusaka. Chronique d'une négociation 
internationale, Cahiers africains, 51-52, Tervuren, Institut Africain-CEDAF, Paris, L'Harmattan, $219 \mathrm{p}$.

- WILLAME J.-C. (2007), Les «Faiseurs de paix» au Congo. Gestion d'une crise internationale dans un Etat sous tutelle, Grip, Bruxelles, Ed. Complexe.

- WILLAME J.-C., voir DE VILLERS G. (1998), MATHIEU P. (1998).

- WILLIAM D.B., LESH R.W. \& STAMM A.L. (1995), Zaire, World Bibliographical Series, volume 176, Clio Press, Oxford, England, Santa Barbara, California, Denver, Colorado, 269 p., un index des auteurs, un index des matières, une carte. - WILUNGULA B. COSMA (1997), Fizi 1967-1986. Le maquis Kabila, avant-propos de Jean-Luc Vellut, Cahiers Africains, 26, Bruxelles, Institut Africain-CEDAF, Paris, L'Harmattan, $136 \mathrm{p}$.

- WIRTHMANN A., voir STACHE N. (1998).

- WOlFF E., MASHINI DHI MBITA MULENGHE J.-CI. \& IPALAKA YOBWA J. (coordination par I-MAGE Consult) (2001), Organisation de l'espace et infrastructure urbaine en République Démocratique du Congo, Les dossiers de l'ADIE (Association pour le Développement de I'Information Environnementale), PRGIE (Programme Régional de Gestion de I'Information Environnementale), $47 \mathrm{p}$.

- WOLFF E., voir DELBART V. (2002), DE SAINT MOULIN L. (2006)

- WOREBEY M., GEMMEL M., TEUWEN D.K. et al. (2008), «Direct evidence of extensive diversity of HIV-1 in Kinshasa by 1960", Nature, 455, 7213, pp. 661663.

- YEMBA POYO TOMBELE (1999), «Les frontières internationales et les limites provinciales du Katanga», Congo-
Afrique, 332, pp. 103-120.

- YOKA L. M. (1995), Lettres d'un Kinois à l'oncle du village, Coll. Zaïre, Années 90, volume 5, Cahiers Africains, 15, Bruxelles, Institut Africain-CEDAF, Paris, L'Harmattan, $173 \mathrm{p}$.

- YOKA L. M. (1999), Kinshasa, signes de vie, Cahiers Africains, 42, Tervuren, Institut Africain-CEDAF, Paris,

L'Harmattan, $168 \mathrm{p}$

- YU L., voir KUVIBIDILA S. (1993).

- YVOR M., voir BOUVIER P. (1997).

- ZANA AZIZA ETAMBALA M. (1993), In het land van de Banoko: de geschiedenis dan de Kongolese/Zairese aanwezigheid in België van 1885 tot heden, Leuven, Katholieke Universiteit Leuven, Hoger Instituut voor de Aarbeid, Coll. Steunpunt Migranten, 7, $103 \mathrm{p}$.

- ZANA AZIZA ETAMBALA M. (2004), «La question kimbanguiste: de la persécution à la tolérance», in MABIALA MANTUBANGOMA P. (dir.), La Nouvelle Histoire du Congo, Cahiers Africains, 65-66-67, pp. 365-390.

- ZANA AZIZA ETAMBALA M. (2005), "L'Armée du Salut et la naissance de la "Mission des Noirs" " au Congo Belge, 1934-1940", Annales Aequatoria, 26, pp. 67-164.

- ZANA AZIZA ETAMBALA M. (2008), De teloorgang van een modelkolonie. Belgisch Congo 1958-1960, Leuven/ Voorburg, Acco, 473 p.

- ZANA AZIZA ETAMBALA M. \& MARYSSE S. (éd.) (1995), «C.D.I.BWAMANDA (Zaïre). Un modèle de développement intégral en milieu rural? Een N.G.O.-model van integrale streekontwikkeling in Zaïre?», Africa Focus, 11, 14, 161 p.; textes bilingues: préface de Zana Aziza Etambala et S. Marysse.

- ZEILIG L., voir RENTON D. (2007). 


\section{PLAN DES MATIÈRES}

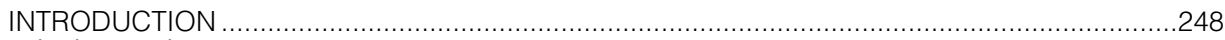

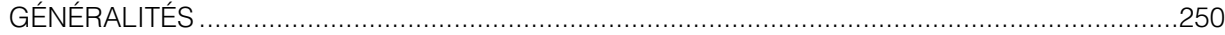

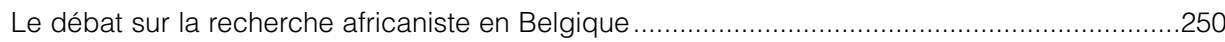

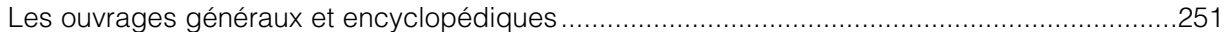

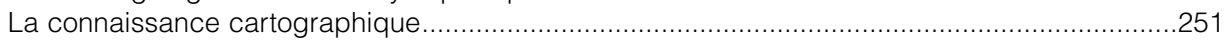

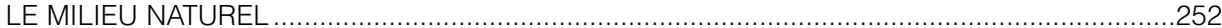

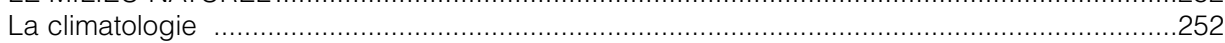

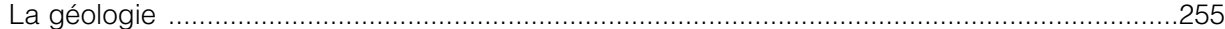

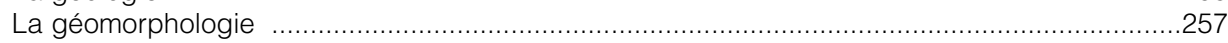

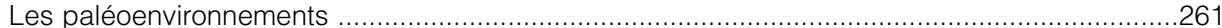

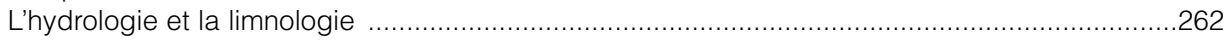

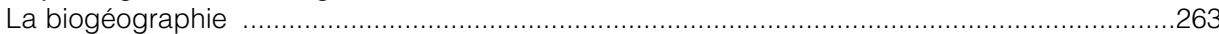

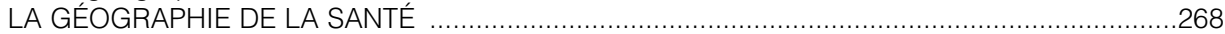

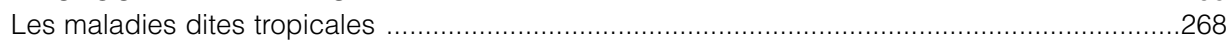

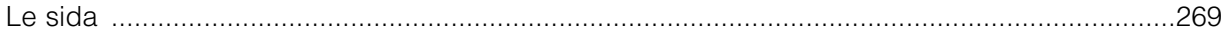

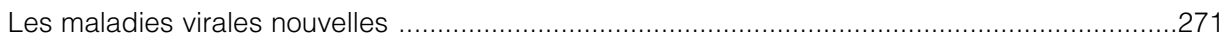

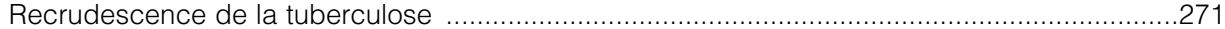

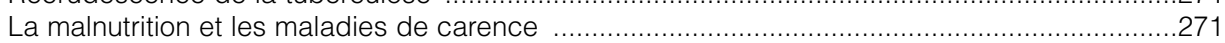

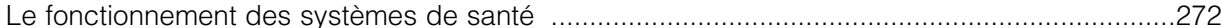

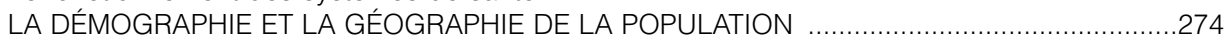

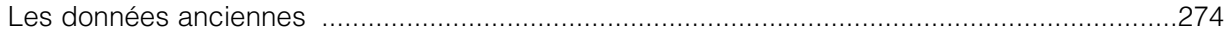

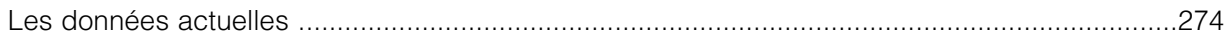

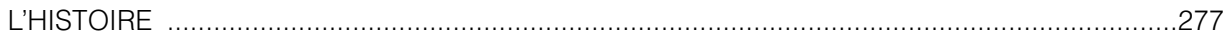

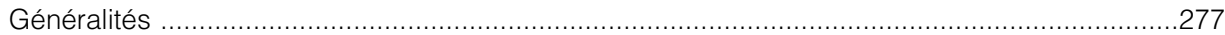

L'histoire précoloniale

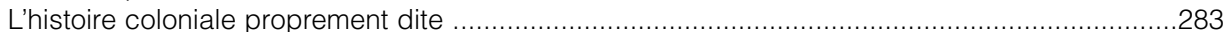

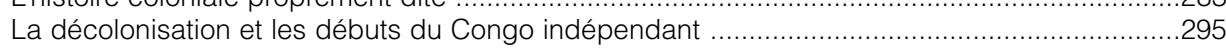

Les événements des deux dernières décennies ....................................................................299

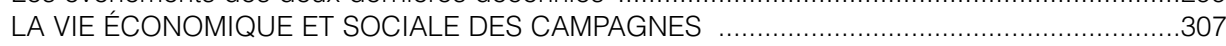

Les groupes ethniques traditionnels et leurs techniques …….........................................307

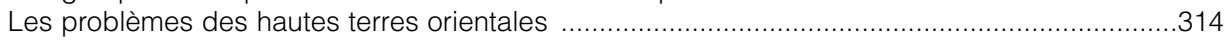

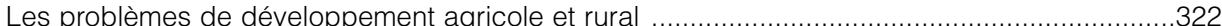

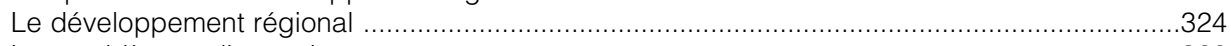

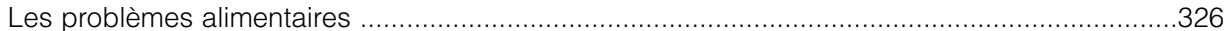

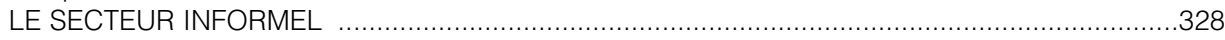

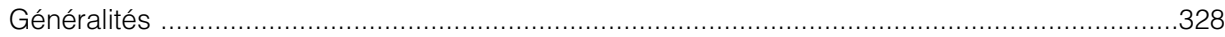

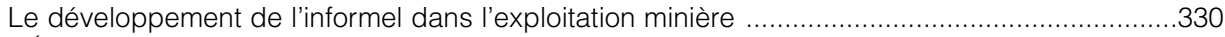

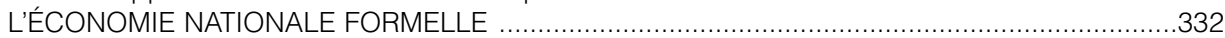

L'évolution économique ...............................................................................

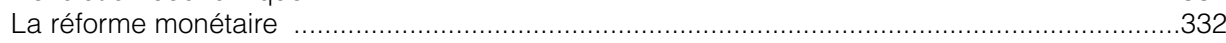

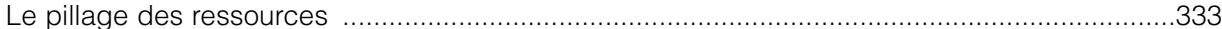

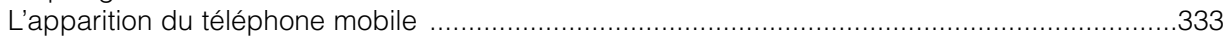

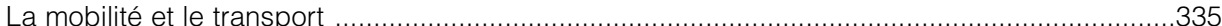

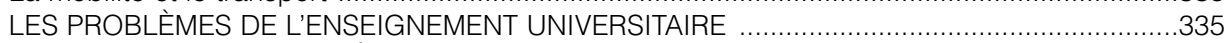

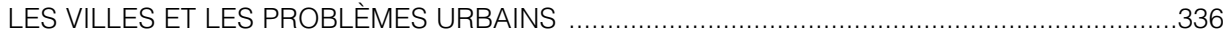

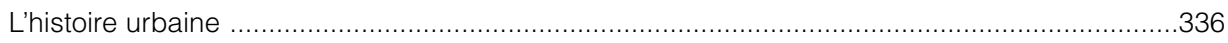

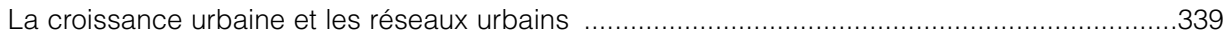

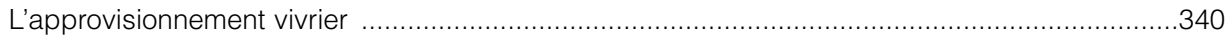

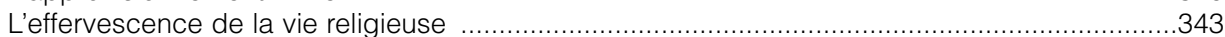

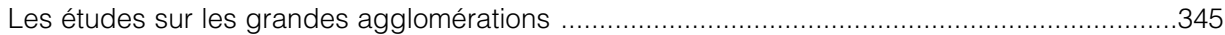

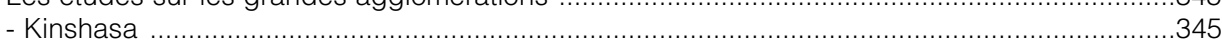

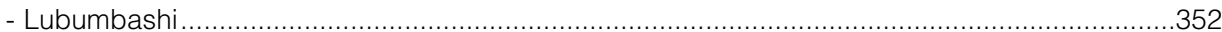

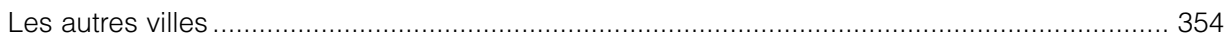

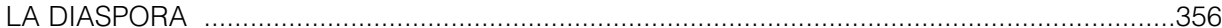

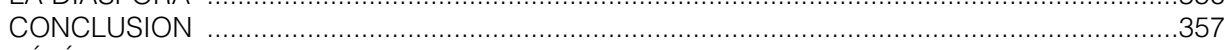

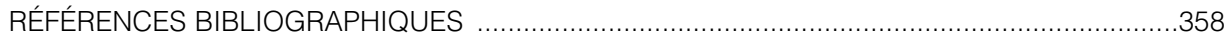

